\title{
NUMERICAL SIMULATION OF THE FLOW THROUGH AN AXIAL TIDAL-CURRENT TURBINE EMPLOYING AN ELASTIC-FREE-SURFACE APPROACH
}

\begin{abstract}
Dissertation presented to the Escola Politécnica of the University of São Paulo for the degree of Master of Science in Engineering
\end{abstract}

São Paulo

2018 


\section{NUMERICAL SIMULATION OF THE FLOW THROUGH AN AXIAL TIDAL-CURRENT TURBINE EMPLOYING AN ELASTIC-FREE-SURFACE APPROACH}

Dissertation presented to the Escola Politécnica of the University of São Paulo for the degree of Master of Science in Engineering.

Concentration Area:

Naval Architecture and Oceanic Engineering

Advisor:

Prof. Dr. Gustavo Roque da Silva Assi

São Paulo

2018 
Este exemplar foi revisado e alterado em relação à versão original, sob responsabilidade única do autor e com a anuência de seu orientador.

São Paulo, 14 de agosto de 2018

Assinatura do autor

Assinatura do orientador

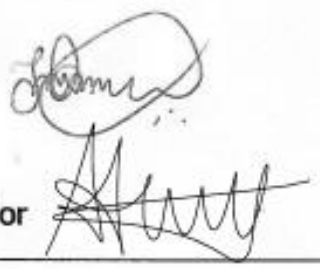

Catalogação-na-publicação

Mattavo, Fernando

Numerical Simulation of the Flow Through an Axial Tidal-Current Turbine Employing an Elastic-Free Surface Approach / F. Mattavo -- versão corr. -São Paulo, 2018.

$152 \mathrm{p}$.

Dissertação (Mestrado) - Escola Politécnica da Universidade de São Paulo. Departamento de Engenharia Naval e Oceânica.

1. MECÂNICA DOS FLUÍDOS COMPUTACIONAL, FONTES RENOVÁVEIS DE ENERGIA, ENERGIA MARÉMOTRIZ, TURBINAS HIDRÁULICAS I.Universidade de São Paulo. Escola Politécnica. Departamento de Engenharia Naval e Oceânica II.t. 


\section{Nome: MATTAVO, Fernando}

Título: Numerical Simulation of the Flow Through an Axial Tidal-Current Turbine Employing an Elastic-Free Surface Approach

Dissertação apresentada à Escola Politécnica da Universidade de São Paulo para a obtenção do título de Mestre em Engenharia

Aprovado em: 15/06/2018

Banca Examinadora

Prof. Dr. Gustavo Roque da Silva Assi

Instituição Escola Politécnica da Universidade de São Paulo

Julgamento Aprovado

Prof. Dr. Fábio Saltara

Instituição Escola Politécnica da Universidade de São Paulo

Julgamento Aprovado

Prof. Dr. $\quad$ Reinaldo Marcondes Orselli

Instituição Universidade Federal do ABC

Julgamento Aprovado 


\section{Dedicatory}

First of all, I dedicate this work for my mother, Maria de Lourdes, who fulfilled me with love and gave all the support I have needed in my whole personal, professional and academic life, fulfilling me with courage and enthusiasm to face the most difficult challenges.

I also remember my colleagues and professors from Universidade de São Paulo, who made possible the conclusion of this work and are fundamental parts of my academic journey. Specially, I would like to dedicate this work for my advisor PhD Gustavo Assi, who helped me in critical moments of this work and gave me fundamental insights for the continuity of the development of my Master Degree. I would like to dedicate also this work for the Professor Celso Pesce, being fundamental for the development of my critical sense and analytical personality, inspiring me for being an open minded person who always looks for knowledge.

I would like to dedicate this work to my colleagues from Voith Hydro Ltda., who gave me support in my professional career, contributing to my personal evolution since April of 2013. I have a special dedicatory for Ph.D. Humberto Gissoni, who gave me the opportunity and the incentives always to keep developing myself, for Leonardo Silva, who supported me and in difficult moments of my life, showed interest in helping, being a valuable friend, for MSc. Leandro Bernardes, who always supported me and inspired me for the scientific development and for all other colleagues that helped me in my professional career.

Finally, I dedicate this work for all of my Friends, who always believed me and were fundamental parts from my life, even in the difficult moments, when they were at my side to guide me through the most challenging paths from my personal and professional life. 
"I am just a child who has never grown up. I still keep asking these 'how' and 'why' questions. Occasionally, I find an answer".

(Stephen Hawking) 


\section{Resumo}

O crescimento econômico mundial e o aumento na demanda pela geração de energia andam juntos. No entanto, uma maior capacidade de produção de energia poderia afetar negativamente o meio ambiente. Mesmo as fontes limpas e renováveis, como a hidrelétrica e a eólica acarretam em impactos socioeconômicos e ambientais. Por exemplo, a construção de uma usina hidrelétrica demanda uma imensa área alagada que pode devastar florestas inteiras e a instalação de uma usina eólica pode afetar a migração de certas espécies de pássaros e produzir altos níveis de barulho.

Portanto, para equilibrar as vantagens e desvantagens devidas a cada meio de produção de energia, é necessária a diversificação, que demanda de investimentos em novas fontes. Neste contexto, a geração de energia nos oceanos é destacada. O primeiro ponto a respeito desta fonte é de que não há a necessidade de remoção da população na área de instalação, tal como os métodos de geração dentro do continente. O segundo principal ponto é a respeito da distribuição de energia. A maior parte da população mundial vive em regiões costeiras, diminuindo, portanto, a distância entre a produção e demanda, reduzindo assim, seus custos.

As duas principais metodologias para se explorar a energia proveniente dos oceanos são: Energia de Ondas e Energia de Marés. E considerando que os ciclos de mare são governados principalmente pela interação gravitacional entre os oceanos, lua e sol, eles são facilmente previsíveis, o que aumenta a confiabilidade dos sistemas de geração de energia baseados em marés.

Este trabalho explora as metodologias para analisar a geração de energia a partir de uma única turbina axial de corrente de maré através de uma metodologia baseada nas equações de Navier-Stokes com a média de Reynolds, analisadas em regime permanente. São discutidos efeitos da direção do escoamento, perfil de velocidades na entrada e nos níveis de turbulência. Os resultados são comparados com experimentos.

É proposta uma metodologia alternativa para a modelagem da superfície livre com CFD uma vez que a metodologia atual é baseada em um escoamento 
bifásico que demanda de um refinamento adicional da malha e é computacionalmente caro. A nova metodologia usa uma parede elástica na região da superfície livre com a rigidez ajustada para se obter o mesmo efeito de restauração que a gravidade.

De maneira geral, os resultados para o domínio aberto se aproximaram dos resultados experimentais, validando o modelo numérico e além disso, o modelo considerando confinamento da turbine mostrou maiores valores para os coeficientes de potência e empuxo, estando portanto, de acordo com a teoria do disco atuador.

O modelo com a superfície livre elástica apresentou problemas de convergência, relacionados com números de Froude elevados, uma vez que isto se relaciona com maiores deformações na região da superfície livre. Uma simulação com $10 \%$ da velocidade original foi realizada, obtendo-se resultados coerentes para ambos coeficientes de potência e empuxo.

Palavras-Chave: Energia do Oceano, Turbinas de Corrente de Maré, Dinâmica dos Fluídos Computacional (CFD), Modelagem de Superfície Livre 


\section{Abstract}

Together with the world economic growth is the increasing of energy generation demand. However, the upgrade of world power production capability could affect the environment negatively. Even the clean and renewable sources, such as hydroelectricity and wind powers have socio-economic and environmental disadvantages. For example, the required flooded area for a hydro power plant construction could devastate entire forests, and the installation of a wind farm power plant could affect migratory rotes of birds and generate high levels of noise.

Hence, for the balancing of advantages and disadvantages of each power generation source, it is necessary to diversify, which requires investments in new power sources. In this context, the energy generation in the ocean is highlighted. The first point concerning the ocean energy is that there is no need of population removal from the installation area, such as the onshore based methods and the second point is that most of the population is concentrated in coastal areas. Therefore the production occurs near to the demand, decreasing the costs with energy distribution.

The two main methodologies for harassing energy from oceans are based on gravity waves and in tides. And since the tidal cycles are governed mainly by the gravitational interaction between oceans, Moon and Sun, they are easily predictable, which increases the reliability of such systems.

These works explores methodologies to analyse the power generation from a single axial tidal current turbine through a Steady State RANS methodology. Are discussed the effects of flow directionality, inlet velocity profile and turbulence levels and the results are compared with an experimental scheme.

It is proposed an alternative methodology for free surface modelling in the CFD analysis. The usual methodology, VOF, it is based on a homogeneous, biphasic approach which requires an additional mesh refinement and is computationally expensive. This new methodology introduces an elastic wall approach in the free surface region in which the stiffness is calculated to provide the same restoring effect as gravity. 
In general, the results for open domain matched with the experimental results, validating the numerical model and the confined domain has shown a higher power and thrust coefficients if compared with the open domain, which is in accordance with the actuator disk theory approach.

The elastic free surface presented convergence problems related to high Froude numbers and therefore to high deformations. However, a simulation with $10 \%$ of the original inlet velocity was performed, achieving reasonable results for both power and thrust coefficients evaluation.

Key Words: Ocean Energy, Tidal Current Turbines, Computational Fluid Dynamics (CFD), Free Surface Modelling 


\section{Contents}

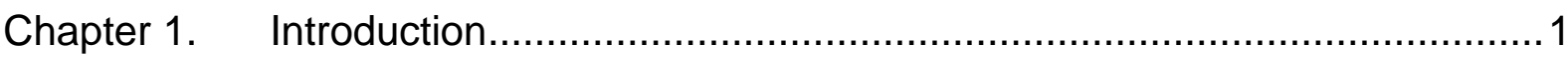

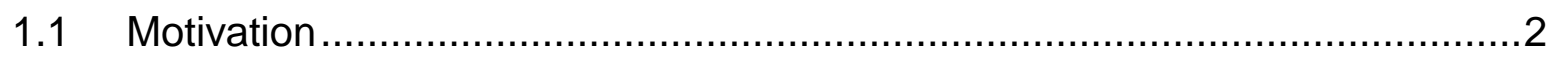

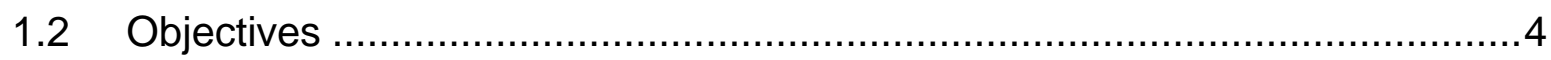

1.3 Text Structure

Chapter 2. World Energy Matrix and Renewable Energy Generation ....................8

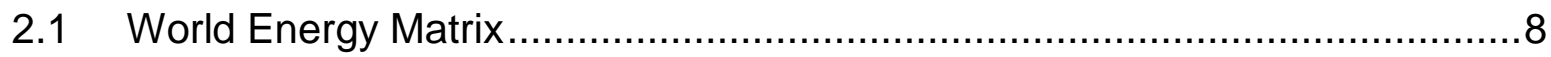

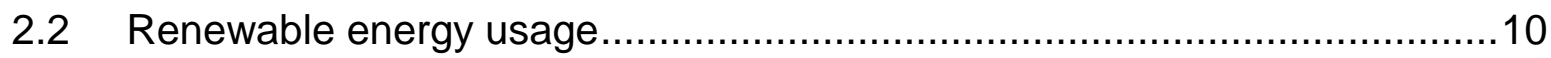

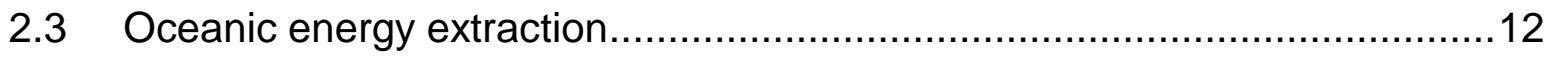

Chapter 3. Literature review on tidal axial turbines ………............................. 15

3.1 Tidal Current Turbines......................................................................15

3.1.1 Experimental Analysis ..............................................................15

3.1.2 Computational Fluid Dynamics Analysis ..........................................20

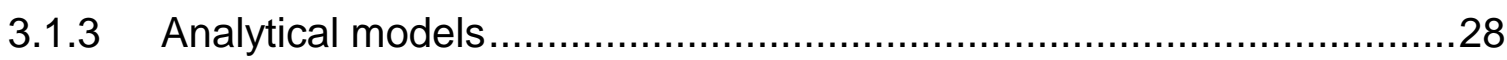

3.1.4 Tidal farm and wind farm optimization methods................................29

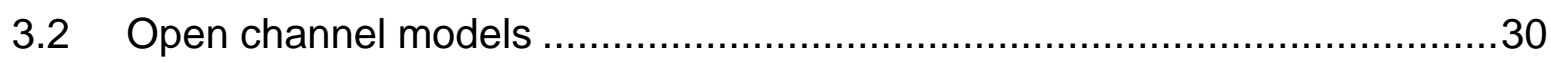

Chapter 4. Theoretical Fundamentals .......................................................... 32

4.1 Basics equations of Fluid Dynamics........................................................33

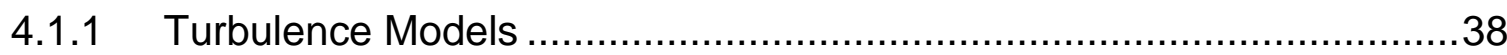

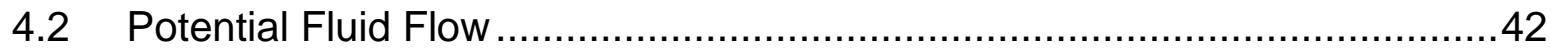

4.3 Solid Mechanics for Orthotropic Bodies …….......................................4

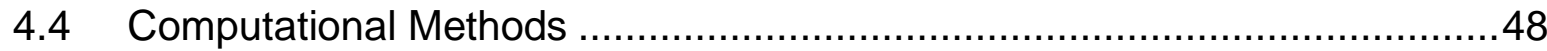

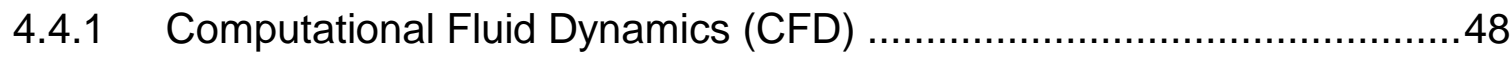

4.4.2 Finite Element Method ...................................................................

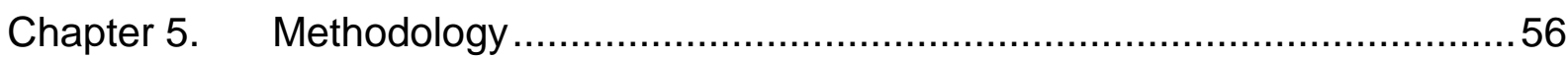

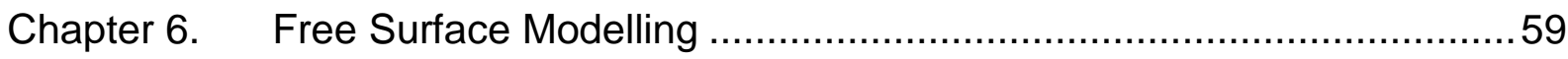




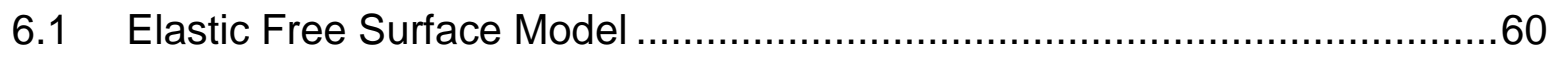

6.1.1 Orthotropic Solid Transversal Stiffness Calculation ..............................60

6.2 Consequences in fluid flow due to substitution of buoyancy for elastic free surface .63

6.2.1 Waves due to a Submerged Cylinder .................................................63

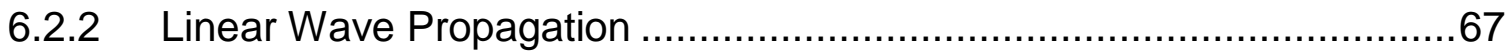

6.2.3 Actuator Disk Model for an Open Channel ........................................73

6.3 Steady State Finite Element Model from Elastic Free Surface .....................76

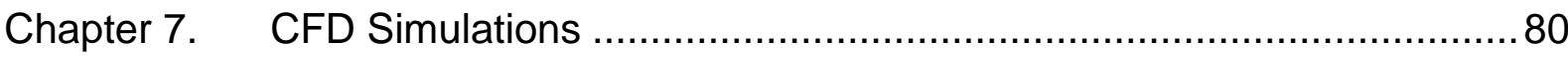

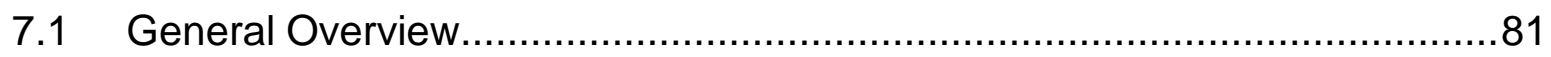

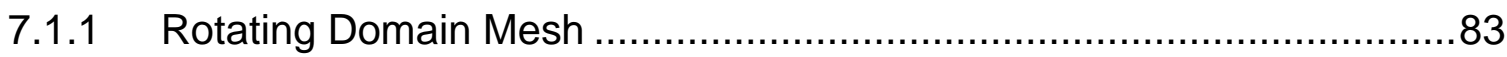

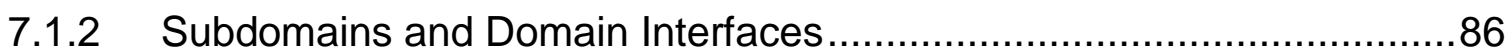

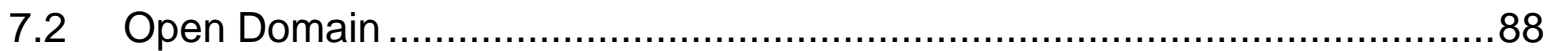

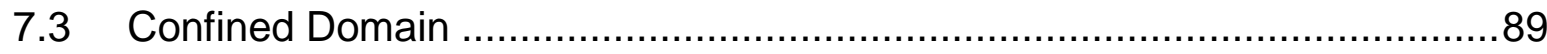

7.4 Open Channel Model - Elastic Free Surface ….........................................89

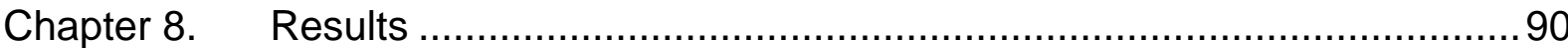

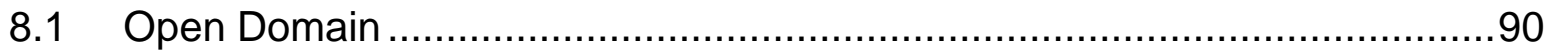

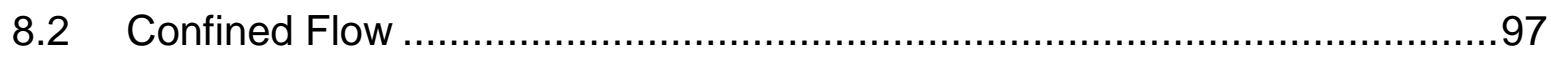

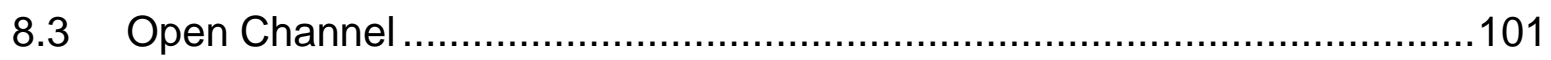

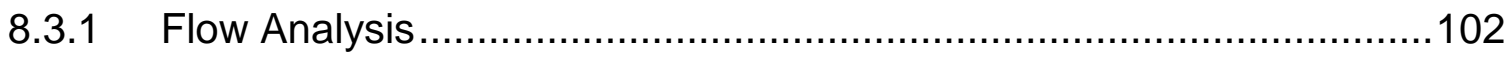

8.3.2 Deformations in the Solid Model ....................................................109

8.4 Comparison Between the CFD Models …….....................................111

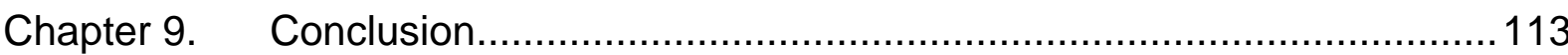

Chapter 10. Proposals for complementary works ….....................................115

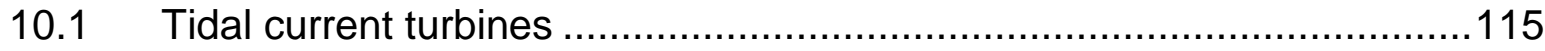

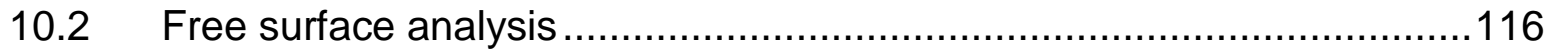

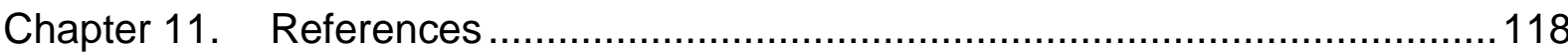


ATTACHMENT A - Potential Fluid Flow 126

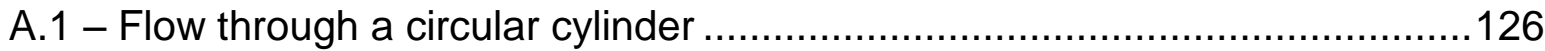

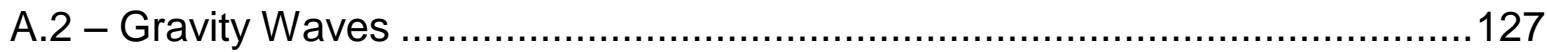

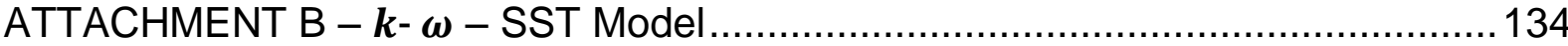

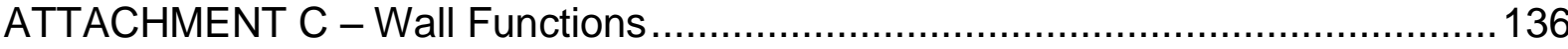

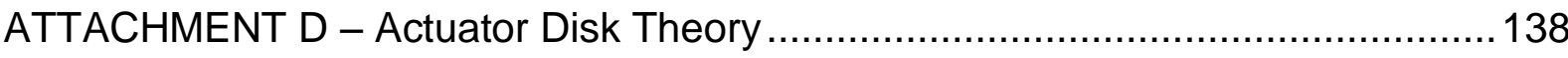

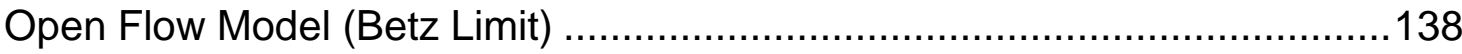

Confined Flow Model (Ducted Flow) ....................................................141

Single Turbine Placed on Open Channel Flow ..........................................144

ATTACHMENT E - Tidal Current Turbines ........................................................... 147

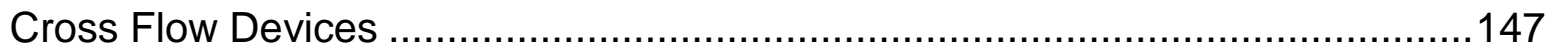

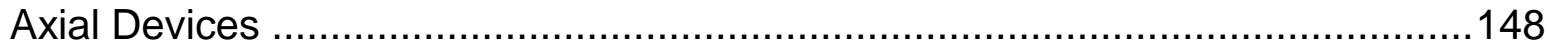

ATTACHMENT F - Finite Volume Elements ......................................................... 150

Finite Volume Method for Unstructured Meshes........................................150 


\section{Chapter 1. Introduction}

All environmental and economic researches point to clean and renewable energy resources. It is not allowable that in XXI Century to still insist on energy generation matrix based on thermoelectric power plants. Even the most known industry for its dependency on fossil fuel, the automotive industry, has investments in researches to substitute the traditional combustion-based motors with electric ones, with more autonomy and efficient. The Figure 1 shows the evolution of the number of the electrical cars stock since 2010. The shape of the curve reveals its ascendant behavior and the most pessimist prediction points that in 2030, at least 60 million electric vehicles will be in the street (International Energy Agency, 2017).

Furthermore, several works highlight the direct dependence between a country economic growth and its capability of energy generation. In China, was performed an empirical assessment correlating the economy evolution with the total energy consumption and its $\mathrm{CO}_{2}$ generation (Wang, Li, Fang, \& Zhou, 2016). Considering that several countries are still underdeveloped and even the developed countries still have its economy growing up, the current world electric energy production, mainly based on fossil fuel, will contribute raising the $\mathrm{CO}_{2}$ emission, which is already in a worrisome level.

Therefore, is concluded that is expected the growth of the world economy which leads to an increase in the energy production. Causing impacts on the world climate and the environment. Based on this, the need for researches and development in clean energy generation is mandatory for sustainable development.

For a clean energy generation, there are several methods, most of them converting the available energy on nature into electrical power, such as wind power plants that convert the kinetic wind energy and a hydroelectric power plant that converts the potential gravity energy stored in water reservoirs. Each energy source with its advantages and problems. 
Figure 1 - Number of electric cars in the world since 2010

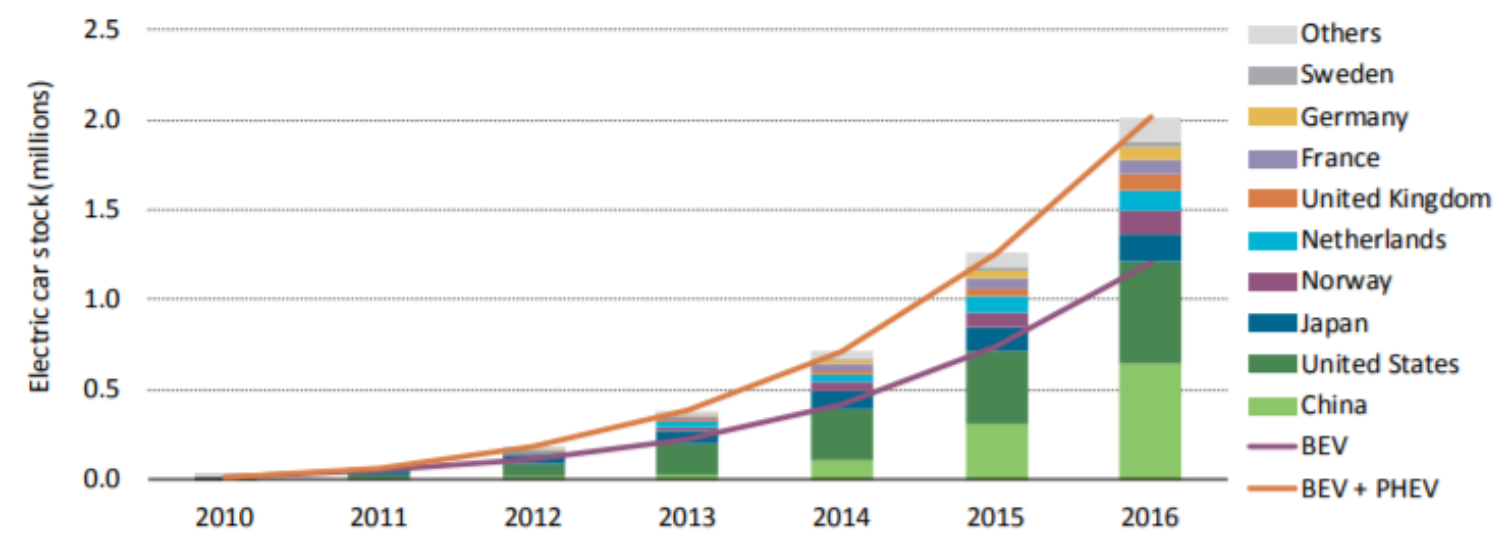

Source: (International Energy Agency, 2017)

A known source of renewable energy it is the ocean. There is available power in several ways, such as in surface waves, salinity and temperature gradients and due to tides. An important factor from oceanic energy extraction lies in the early development stage with prototypes and small power plants that only could attend a local demand.

Moreover, this work focuses on the extraction of tidal energy, in the form of kinetic energy due to underwater currents, by placing axial turbines. The main goal is doing a hydrodynamic performance assessment studying several factors, such as interaction with the free surface, turbulence, and proximity with physical boundaries and another neighbor turbine, by a Computational Fluid Dynamics approach., mainly studying a most computational method for evaluating free surface effects on turbine performance.

\subsection{Motivation}

The tidal based energy still need researches focusing on its performance enhancement, since its generation cost is prohibitive if compared to other renewable sources (IRENA, 2014). Furthermore, the environmental impacts still under evaluation since are not completely known the impacts of the turbines deployment on marine relief, sedimentation, fauna, and flora.

For both issues, a most accurate flow modelling is useful, since it allows improving the individual turbine efficiency and the whole farm performance and permits to evaluate the changes in local flow pattern which could affect the 
seabed relief with impacts on vegetation and in animals that live near to the installation point.

The complete modelling of flow through a tidal current generation farm includes full modelling of turbine blades and structure, original velocity profile, and turbulence levels, channel geometry, free surface effects as its blockage and the gravity waves, the interaction between the turbines and maritime currents that could affect flow directionality. Moreover, even for neglecting any effect, is essential to comprehend its influence in the hydrodynamic performance, such as power generation, and in other flow parameters such as wake length and recovery. However, a model including all described flow parameters is computationally prohibitive, even on supercomputers.

An interesting parameter is the free surface effect on tidal current turbine hydrodynamic performance and in mechanical loading. Its traditional modelling uses a biphasic fluid flow with a new flow variable that is the proportion between the two fluids. To guarantee the convergence, without numerical diffusion problems and with a reasonable free surface resolution, it is necessary a high refinement which increases the computational costs.

Besides that, other industrial flows, such as flow around risers, oil platforms, and other oceanic engineering applications, the free surface effects are not negligible, and therefore, such problems demand more efficient ways of free surface modelling.

Hence, the work is focused on flow analysis through an axial tidal current turbine with special attention in free surface effects, including an artificial FluidStructure Approach to perform a steady-state analysis including free surface blockage effects. This method is based on energy conservation and applied to classical fluid mechanics problems such as free surface gravity waves and free surface deformation due to the potential flow through a circular cylinder produces the same results from the original theory. 


\subsection{Objectives}

Considering the analysis of the flow through an axial tidal current turbine close to a free surface using Computational Fluid Dynamics. It is necessary to validate a CFD model with accurate results regarding the experimental ones and then is necessary to validate and implement a free surface analysis model.

Hence, the first objective of this present work is to define and understand the flow through the turbine with the simplest domain: The Domain without lateral boundaries. As seen in the section 4.1.1 there is a set of experimental results corrected for this domain that could be used as a reference to validate some CFD parameters such as domain dimensions, turbulence model and mesh refinement level.

However, both experiment and prototype do not consist of a single turbine placed on an open domain. There is a confinement level which could increase the turbine power and wake recovery length. Hence, the confinement effect is studied in the present work.

For studying such effect, a simulation with slip-free walls in the same dimensions as the test rig presented in the section 4.1.1 is performed with the objective of understanding how such confinement affects the flow.

Additionally, tidal current turbines are subjected to free surface effects. The free surface confines the flow, but less than a slip-free wall, since its deformation allows a higher portion of fluid deviates the turbine disk. Accordingly, it is necessary to perform a simulation including such effect. However, it is necessary to highlight the lack of a computationally efficient methodology for analyzing such effect. For example, the Volume of Fluid method requires a biphasic simulation including an air domain and an extra refinement in the free surface region. Then, in the present text, an alternative methodology is proposed. The new methodology for analyzing free surface is based on energy conservation and uses an orthotropic solid to have a spring restoring effect in such region. Therefore, this work focuses on present the theoretical foundation of such methodology and implements it for the Tidal Current Turbine Analysis. 


\subsection{Text Structure}

As discussed in the previous section, this work is focused on the introduction of an alternative methodology to evaluate the free surface effects in a Computational Fluid Dynamics simulation applied to an axial tidal current turbine. Therefore, there is a necessity on reviewing the basics of fluid dynamics and computational methodology used for solving such kind of problem. Besides, since there is a new proposed methodology, is necessary to present the theoretical development which justifies its usage in the presented problem. And finally, an introduction to the actual scenario and predictions about world energy generation is necessary so that this research is applied in an actual and existing matter.

The Chapter 2 provides an overview of the actual status and predictions regarding the world energy matrix. There is a focus on clean energy, highlighting the main sources and introducing the oceanic energy sources as an alternative to green energy production. Justifying the investment necessities in energy generation is important.

Introduced the potential oceanic energy sources, the actual stage of development of tidal current turbines is presented, focusing on the methodologies for extracting tidal current energy. In such context, some manufacturers and its commercial products are shown in the Chapter 3.

The Chapter 4 presents the most recent works related to the tidal current turbines. Some experiments are discussed including their methodology and results which provides the data to validate computational methodologies for predicting the power and thrust provided by those turbines. The computational methods that are used for analyzing tidal current turbines are also presented in this chapter. The section provides a brief analysis discussing the accuracy of the results according to the analysis goals and the methodology used for obtaining such results. It is shown a brief introduction in the analytical models, which are fundamental to understand the main flow physics and the influence of some boundary conditions in the fluid flow. Besides, even not being the scope of this work, some articles about tidal current turbine farms optimization are 
discussed since such analysis could lead to a higher power harvesting using a lower number of turbines, decreasing the total cost of a tidal current turbine farm and therefore reducing the energy price. Finally, in this chapter, a discussion about some current methodologies for evaluating tidal current turbines is introduced.

For completing this work, is necessary a comprehension about the physics related to turbulent, viscous and incompressible fluid flow and the main subjects related to the simulation of such flow in a computational environment. Hence, the Chapter 5 introduces the basic equations for fluid flow analysis and introduces the methodologies for analyzing a turbulent flow with accuracy. Besides, a brief discussion about the finite volume method is performed highlighting the main features which could influence the results and computational time of a CFD simulation.

Furthermore, it is included in Chapter 5 the necessary tools for modelling the free surface as an elastic wall. This methodology is implemented in this text as an orthotropic solid coupled to the fluid domain. Hence, the basics of solid mechanics are presented and the necessary hypothesis to guarantee the necessary behavior for modelling a free surface are discussed. And since that this solid domain is solved by the Finite Element Method, the basics of such methodology is presented in the last part of this chapter.

The Chapter 6 presents the methodology which drives the analysis presented in this work. It is performed a study involving different boundary conditions, such as an unconstrained flow, a flow confined by slip-free walls and lastly a flow confined by a free surface modeled as an elastic wall. It is highlighted the simulation goals and the necessary steps for achieving them.

The Chapter 7 presents the development of the elastic free surface methodology from the orthotropic solid modelling to the implementation by the so Finite Element Method. Since the classic boundary condition for free surface analysis is modified using such method, three classical analytical problems are solved and the results obtained by the original methodology, and the proposed alternative are compared and discussed. It is shown that for at least these problems the results are the same. 
The Chapter 8 presented the CFD simulations conducted in this work. The boundary conditions and other features related to a CFD simulation such as domain size, mesh, interpolation interfaces are discussed. The methodology used for coupling the fluid flow with the orthotropic solid mechanics is presented for detailing the implementation of the elastic wall free surface method.

The Chapter 9 presents the results obtained from the CFD analysis with the different boundary conditions. The main analysis is focused on power and thrust generated by the turbine. However, some other features, such as wake recovery length and free surface deformation are discussed.

Finally, the Chapter 10 concludes the works while Chapter 11 proposes suggested complementary works related to this text. 


\section{Chapter 2. World Energy Matrix and Renewable Energy Generation}

The energy extraction from the ocean, especially from the tides, is a kind of renewable energy resource. Therefore, to analyze the impact of the introduction of such technology, it is important reviewing the current status of energy production around the world and the predictions about demand evolution in the next years. This chapter is divided into three main sections. The first section is about the current status of world energy production and the predictions about its demand for the next decades. The second section is destined to review the evolution of the renewables energies around the world and. The last section makes an introduction to the current technologies and researches about oceanic energy extraction.

\subsection{World Energy Matrix}

Unfortunately, the world still depends on the fossil energy. As shown in Figure 2 , as recent as in 2015 , over $60 \%$ of the consumed energy was derived from oil and coal. Although the depletion of fossil resources is an issue that humanity must face by the end of this century, the environmental changes, such as global warm will affect the whole life directly on Earth. For example, the average sea level rises due to ice melting in the poles could submerge most of the coastal cities (Hansen, 2015) and it is known that a great part of the population of the world lives in such areas. Figure 2 also shows that the participation of other renewable energies has increased their share from $0.54 \%$ to $0.89 \%$ between 2005 and 2015 . It represents an increase of almost $65 \%$.

The Figure 3 shows the predictions of energy generation (U.S. Energy Information Administration, 2016) for the next 25 years. The total production of energy is predicted to increase by over $50 \%$ in this period. It is clearly seen that the petroleum share and total production is supposed to decrease in the next years and the coal usage stabilize. Most of all increase of the production is due to the gas, hydropower, wind and other renewable sources. Between 2012 and 2040 , it is predicted that energy from biomass, wastes and from ocean increases from 391 billion kW.h to 1247 billion kW.h (320\% increase). 
Figure 2 - Evolution of the world energy matrix in the past ten years

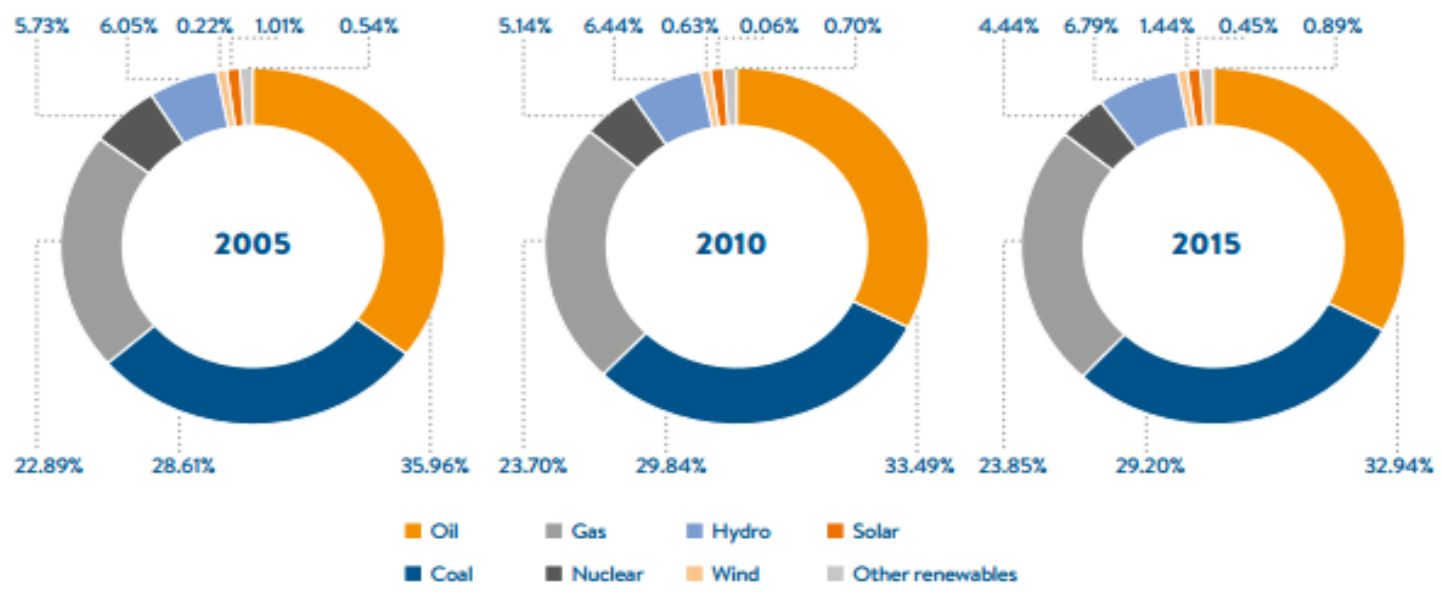

Source: (World Energy Council, 2016)

However, it is important to observe that even in these predictions, coal and natural gasses are still very representative in the world energy matrix. The report by (U.S. Energy Information Administration, 2016) discusses that even in 2040 the transport sector will be strongly dependent on fossil fuels such as gasoline, diesel and jet fuel. Nowadays, the merchant ships, for example, are essentially powered by Diesel. Nevertheless, for general consumption of energy, which includes industrial and domestic usage (in other words, electricity generation), the incentives for renewable resources and alternatives to hydroelectricity are being increased. It is important to diversify the generation and make the sector more reliable and sustainable.

Figure 3 - Electric energy production (left) and renewable energy production in PW.h (right)
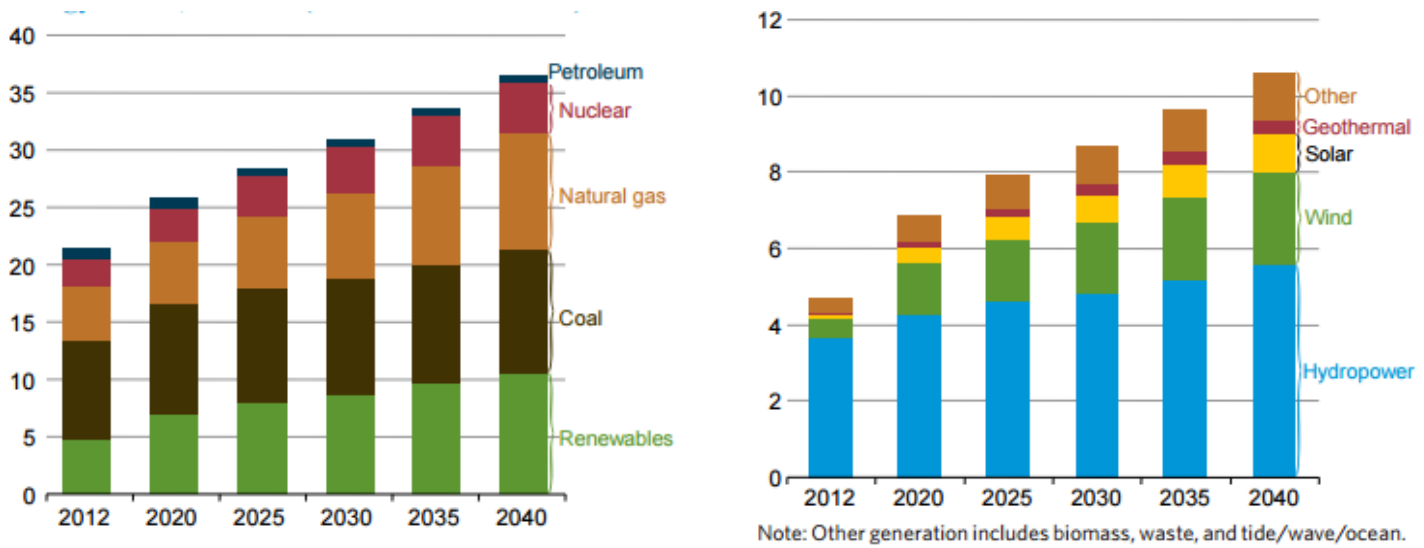

Source: (U.S. Energy Information Administration, 2016) 


\subsection{Renewable energy usage}

As discussed in the previous section, the expected growth of energy production due to renewable sources is about $100 \%$ in the next 25 years. Most of this trend is explained by the pressure for a sustainable process to generate energy. In Brazil, for example, the two last biddings for energy extraction (with its summaries found in (Empresa de Pesquisas Energéticas, 2017A) and (Empresa de Pesquisas Energéticas, 2017B)), shows that in the bidding $A 4 / 2017$, from the $48 \mathrm{GW}$ auction, $99.8 \%$ of the total energy comes from renewable sources, mainly solar resources and in bidding A6/2017, from 53GW auction, $56,1 \%$ comes from renewable resources.

Shown in Figure 3, the renewable energies production is expected to increase by $100 \%$ between 2012 and 2040. Looking to a shorter period, according to the Figure 4, in the USA, the renewable energy share has grown from about 1.9 TW.h, produced in 2006 to 2.8 TW.h, produced in 2016. Even considering the 2008 crisis, it represents an average growth of $4 \%$ yearly, only from renewable resources. The stop of renewable sources expansion in the USA, seen in the projections for 2017 and 2018, is explained due to the new government policies, as explained in (UOL Confere, 2017). These attitudes are severely criticized by specialists since the current $\mathrm{CO}_{2}$ emission rate from the USA shall contribute to increasing the global warming effects, prejudicing the whole world.

Hence, is seen that the world needs to produce more renewable energy, to replace the traditional ones and to increase the total capacity. Besides of that, the hydroelectric source is being criticized due to its high environmental impact, even being a renewable resource and providing grid stability. To solve this problem, is necessarily investing in the diversification of energy production. 
Figure 4 - U.S.A. Energy Production and projections

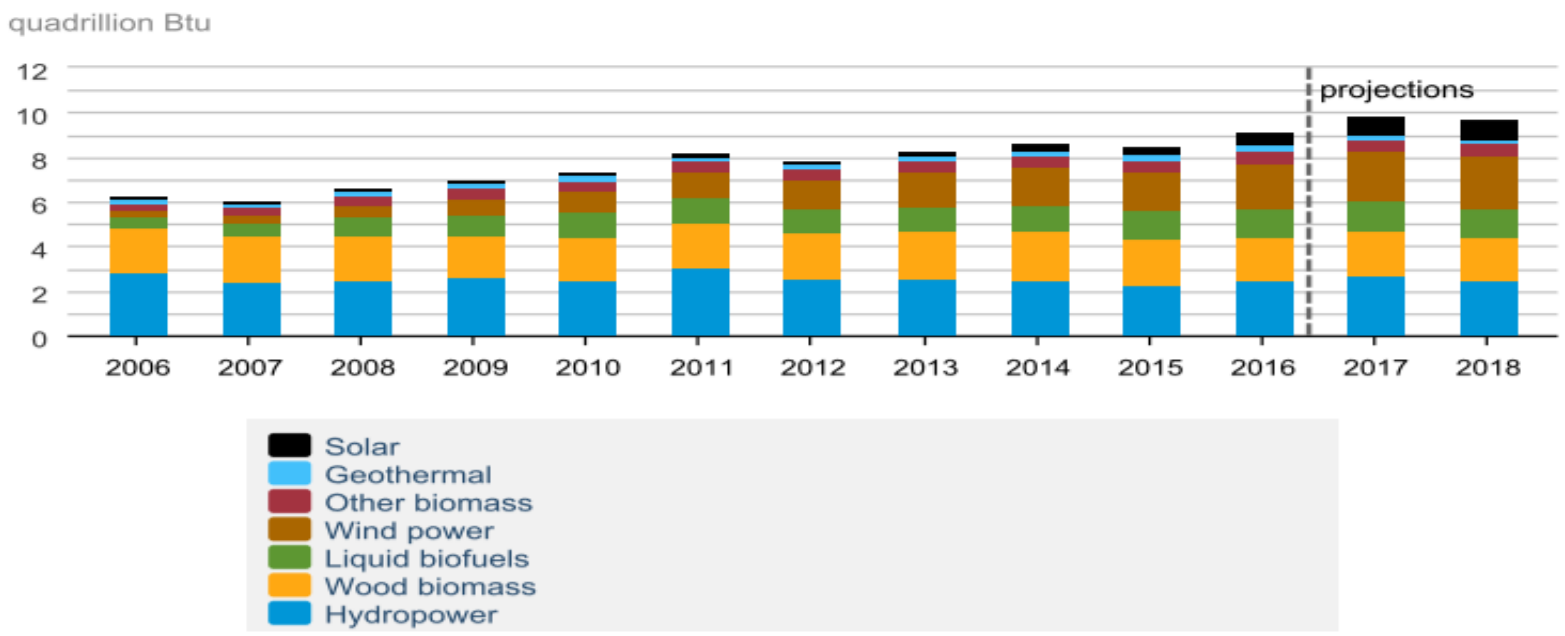

Source: (U.S. Energy Information Administration, 2017)

According to Figure 4 the main renewable sources, in the USA are hydropower, solar and wind power, followed by biofuels. However, even being considered cleaner than the energy generation by fossil fuels, these sources cause environmental and social impacts. Hydropower, for example, requires a large artificially flooded area, affecting the local flora and fauna directly, causing microclimate changes and needing to move the local population to other locations. However, hydro turbines furnish high-quality energy, leading to a stable electric grid being fundamental to systems with variable demand.

Wind power may produce uncomfortable levels of noise and is associated with the death of birds and changing the migratory route of such species (Green Rhyno Energy, 2018). Besides that, the wind is an intermittent source of energy, depending directly on the weather requiring more sophisticated energy conversion system to provide the grid with an acceptable quality power. Therefore, the wind power is important to increase the generation capacity even requiring thermal power plants and hydropower plants to compensate the system oscillations.

The Solar power is the most abundant resource on Earth, it does not produce noise, has low costs of maintenance (since there are not mechanisms as hydro and wind turbines) and could be installed in small scale, such as house rooftops, However, they are also dependent on the weather, such as wind power, and cannot generate energy at night. 
Figure 5 compares the data on the energy production share in the world and Brazil. It is seen that in the world, in a scenario in which the renewable sources participation in the total share is relatively low if compared with Brazil, that more than $60 \%$ of the energy production comes from hydropower plants; Furthermore, the share of other sources is negligible and lower than $2 \%$ in the world. These results, together with the projection shown in Figure 3 indicates the necessity of investing in alternative energy generation, such as the energy from the ocean, which is the focus of this work.

Figure 5 - Share of energy production at the end of 2016

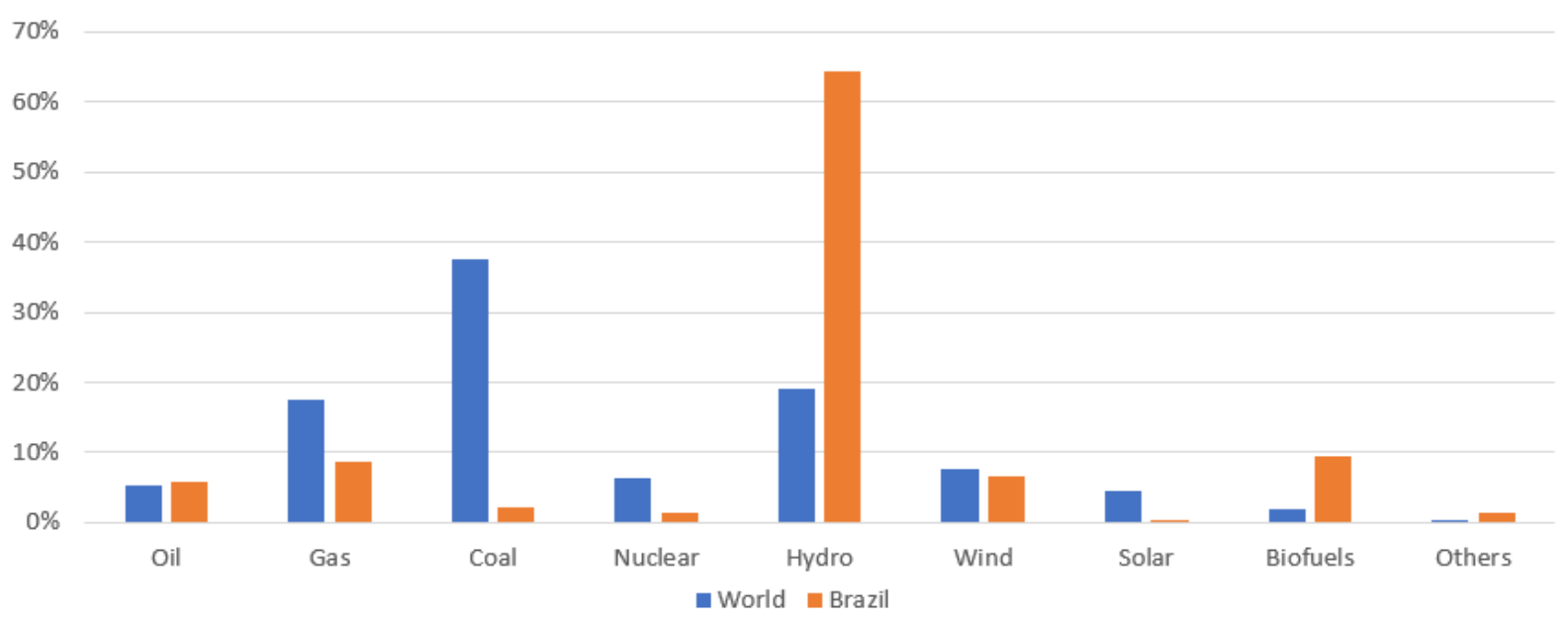

Source: (Ministério de Minas e Energia do Brasil, 2017))

\subsection{Oceanic energy extraction}

The oceanic energy extraction is based mainly on waves and tides. Several methodologies for harvesting wave energy are reviewed in In (Salter et al., 2002) and (Falnes, 2007) and will not be discussed in this work.

According to the IRENA ocean energy technology brief report (IRENA, 2014), there are $1 \mathrm{TW}$ of tidal energy available for harvesting. For comparison, the total capacity of energy production installed in the world at the end of 2016 is 6.5 TW (Ministério de Minas e Energia do Brasil, 2017). In the United Kingdom, for example, there is $10 \mathrm{GW}$ of potential, and the first full scale turbines were deployed in 2012 for tests (TidalEnergy Today, 2015).

Even with this potential, there are some issues regarding to tidal energy exploitation that due to the preliminary phase of the study is not well 
comprehended. According to (Uihlein \& Magagna, 2016), the environmental impacts still under discussion. For example, the impacts of the changes in flow pattern such as differences in sedimentation and hence in the oceanic relief and even the electromagnetic fields due to underwater cables shall be assessed. Another barrier is related to the implementation costs, mainly for the technologies based on tidal currents. The last predictions point on a generation costs of 230 EUR/MW.h, over 10 times above the Sihwa Tidal Power Plant, which sells its energy by $20 \mathrm{EUR} / \mathrm{MW}$.h, indicating that the cost of production must be sharply decreased for a competitive and reliable generation.

In the Figure 6, the main methods are illustrated. The technologies numbered by 1 and 2 on this figure corresponds to the tidal barrages, which stores the elevated water and with turbines similar to that used for low head power plants are used for extracting the energy. The technology numbered by 3 represents a tidal current farm, which is similar to a wind farm, which is the focus of this work and that will be detailed on the section 3 of this text and the technology numbered by 4 is a tidal lagoon, that generates energy by the same mechanisms that the technologies numbered by 1 and 2 .

The first power plant that harassed energy from tides is the La Rance power plant, shown in Figure 7. This power plant is built in France and the civil design uses a dam to store the elevated water and as a bridge, in order to low the costs of the energy generated by this power plant. The turbine section is close to a bulb turbine section, which is a low head turbine, used in power plants such as Santo Antonio, built in the North region of Brazil. A similar solution is used on Sihwa Power Plant, on South Korea, shown in Figure 8. The power plant generates $260 \mathrm{MW}$ of energy, from 10 turbines, in comparison with La Rance that uses 24 turbines to generate $240 \mathrm{MW}$.

However, is important to highlight that the peak power is not constant since the water level occurs due to the tidal mechanism and while the turbines generate power, the reservoir is emptied lowering the available power. 
Figure 6 - Methods for extracting energy from tides

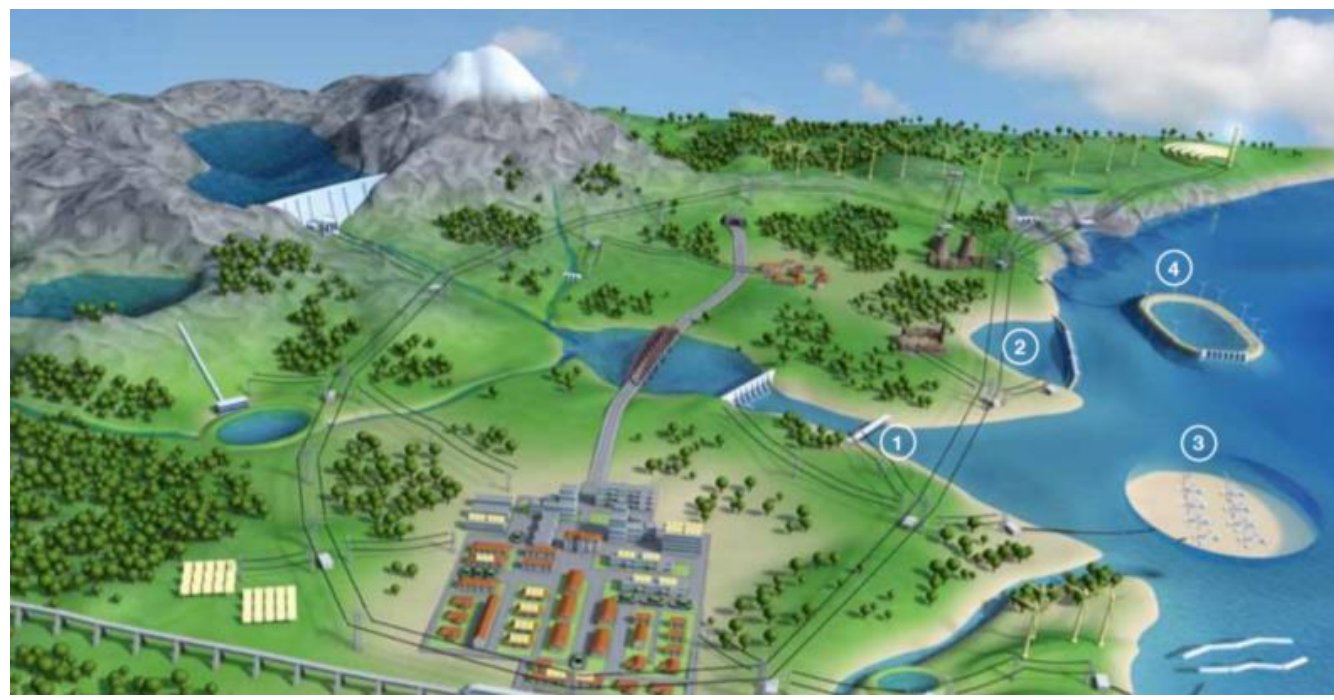

Source: (Extracted from (Andritz Hydro))

Figure 7 - La Rance Tidal Power Plant
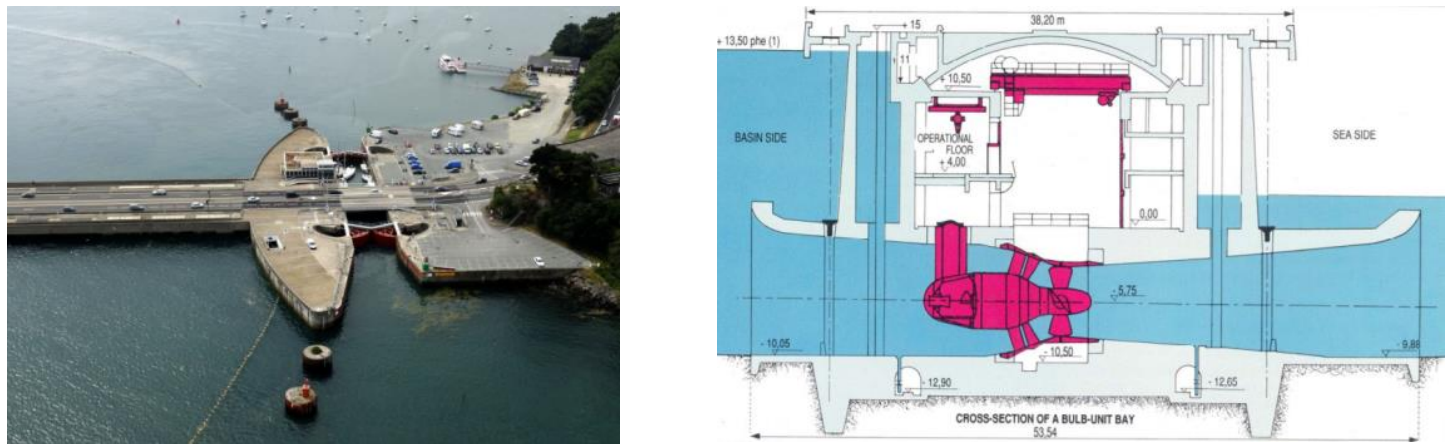

Source: British Hydro (British Hydro, 2009)

Figure 8 - Sihwa Tidal Power Plant Schematic Model
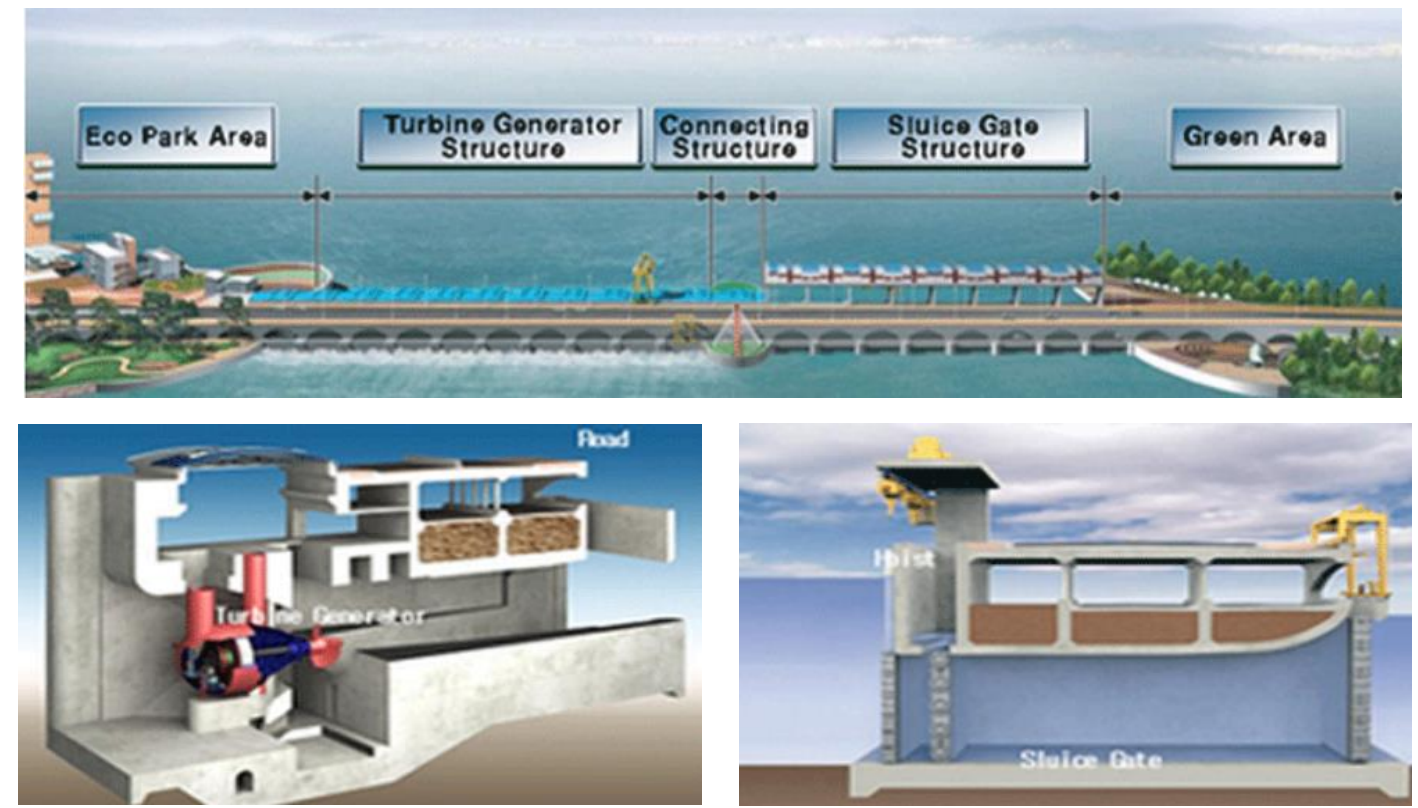

Source: (K Water) 


\section{Chapter 3. Literature review on tidal axial turbines}

This section discusses the axial tidal turbines research status. The most recent works are divided mainly into four groups: Experimental analysis, computational fluid dynamics analysis, analytical analysis, and optimization. Some works present analysis in two or more divisions. Although not being the focus of the text, the optimization methods and the farm layout studies are being extensively explored, therefore, it is briefly mentioned in the present work as a reference. A review of free surface simulation methodologies is also included.

\subsection{Tidal Current Turbines}

\subsubsection{Experimental Analysis}

This text presents a CFD analysis of a tidal axial current turbine. Since a CFD model carries some uncertainties related to the analysed parameters, every analysis shall be validated with experimental analysis. The data of the turbine model used in this text and its thrust and power curve comes from (Bahaj, W.M.J., \& McCann, 2007). The authors made the experimental assessment in an $800 \mathrm{~mm}$ diameter turbine model, with three blades, shown in Figure 9. The experiments were conducted in a cavitation tunnel and a towing tank. The work showed results from thrust and torque measurements and consequently power generated by the turbine in different pitch angles. From the cavitation tunnel, varying the static pressure, the cavitation due to the tip vortex were analysed. The cavitation tunnel has a section of $1.2 \mathrm{~m}$ by $2.4 \mathrm{~m}$ in which the turbine disk presents a blockage of $17.5 \%$ of the channel. The towing tank has a section of $1.8 \mathrm{~m}$ by $3.7 \mathrm{~m}$ in which the turbine provides a blockage of $7.5 \%$ of the channel. The data were corrected for blockage effects according to the procedure found in (Barnsley M.J., 1990). 
Figure 9 - Main instruments and experimental schematic assembly
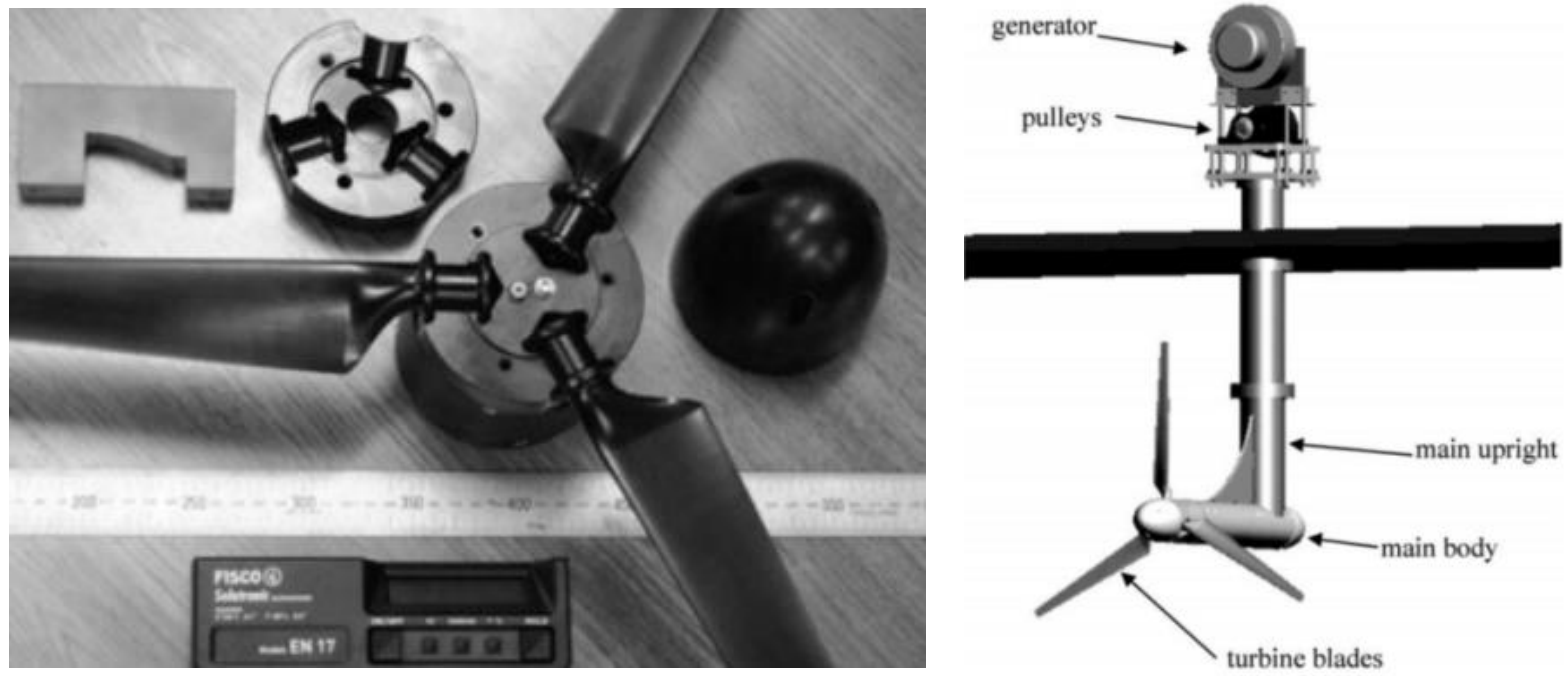

Source: (Bahaj, W.M.J., \& McCann, 2007)

Figure 10 - Schematic Assembly of rotor hub including the torque-thrust dynamometer, bearings and sealings

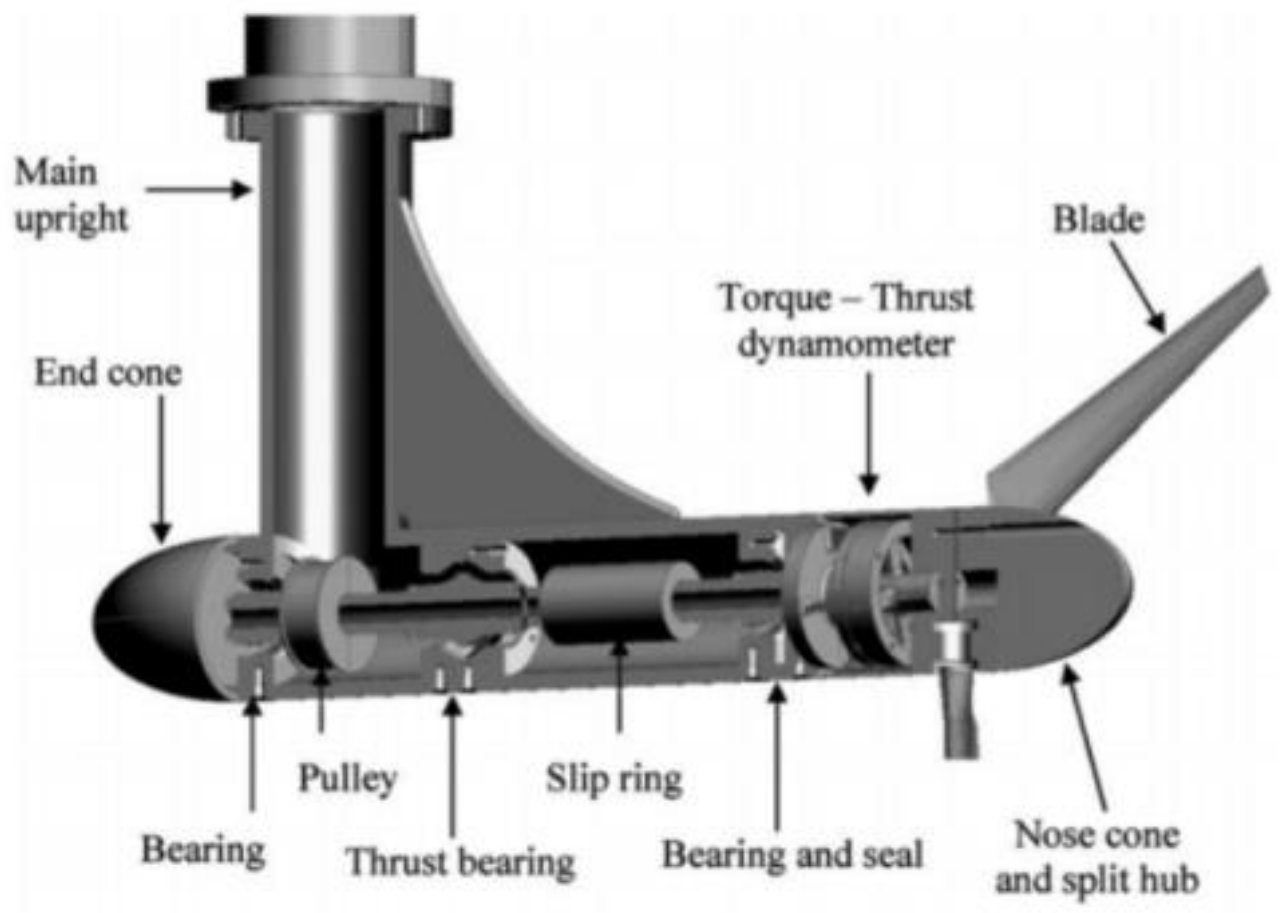

Source: (Bahaj, W.M.J., \& McCann, 2007)

The instrumentation, shown in Figure 10, included a coupled torque-thrust dynamometer, assembled in the turbine shaft before the sealing. It reduces the measured losses. The maximum thrust measurement is $1000 \mathrm{~N}$, and the maximum torque is $50 \mathrm{~N} . \mathrm{m}$, and the uncertainties are respectively $5 \mathrm{~N}$ and $0.25 \mathrm{~N} . \mathrm{m}$. 
Figure 11 - Data from experiments carried out on the cavitation tunnel - Power Coefficient

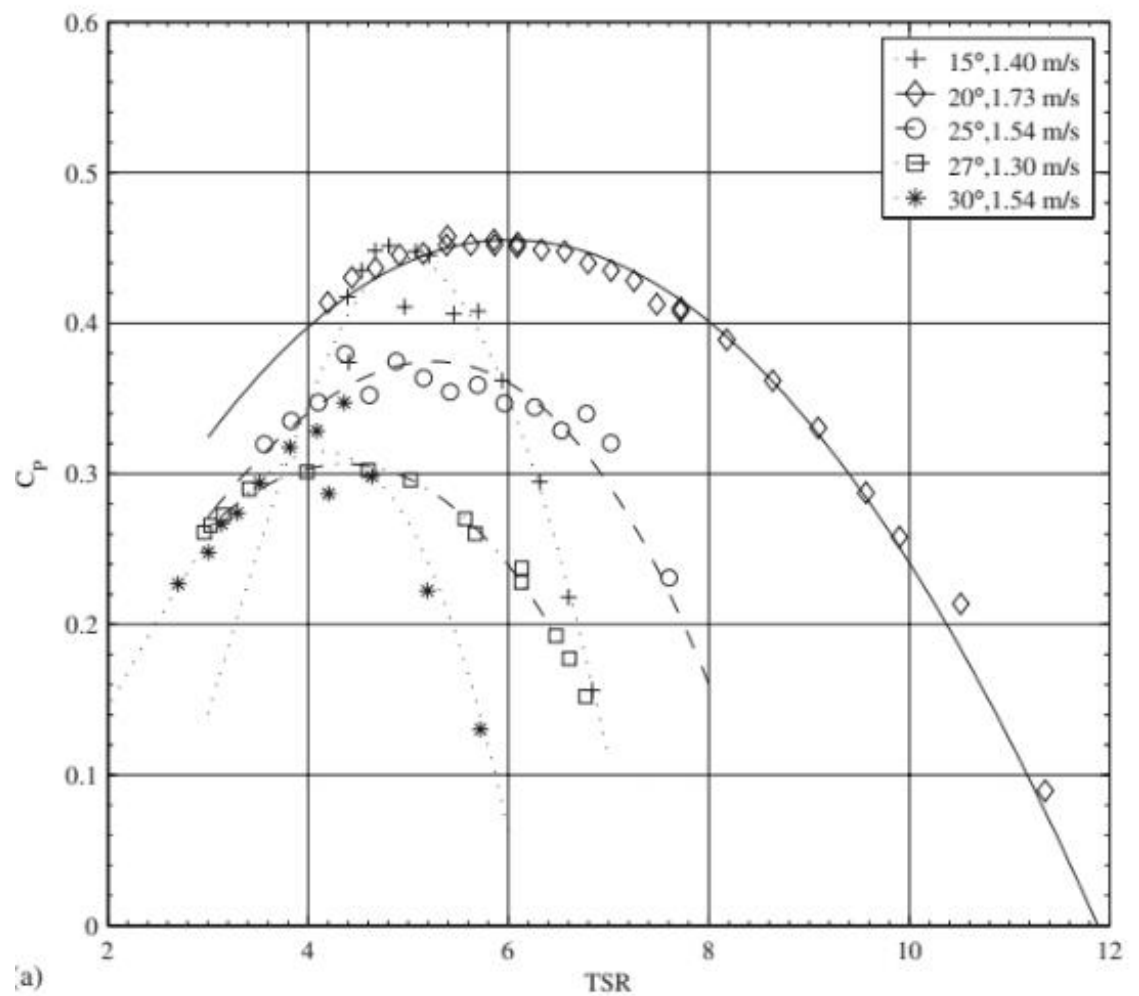

Source: (Bahaj, W.M.J., \& McCann, 2007)

Figure 12 - Data from experiments carried out on the cavitation tunnel - Thrust Coefficient

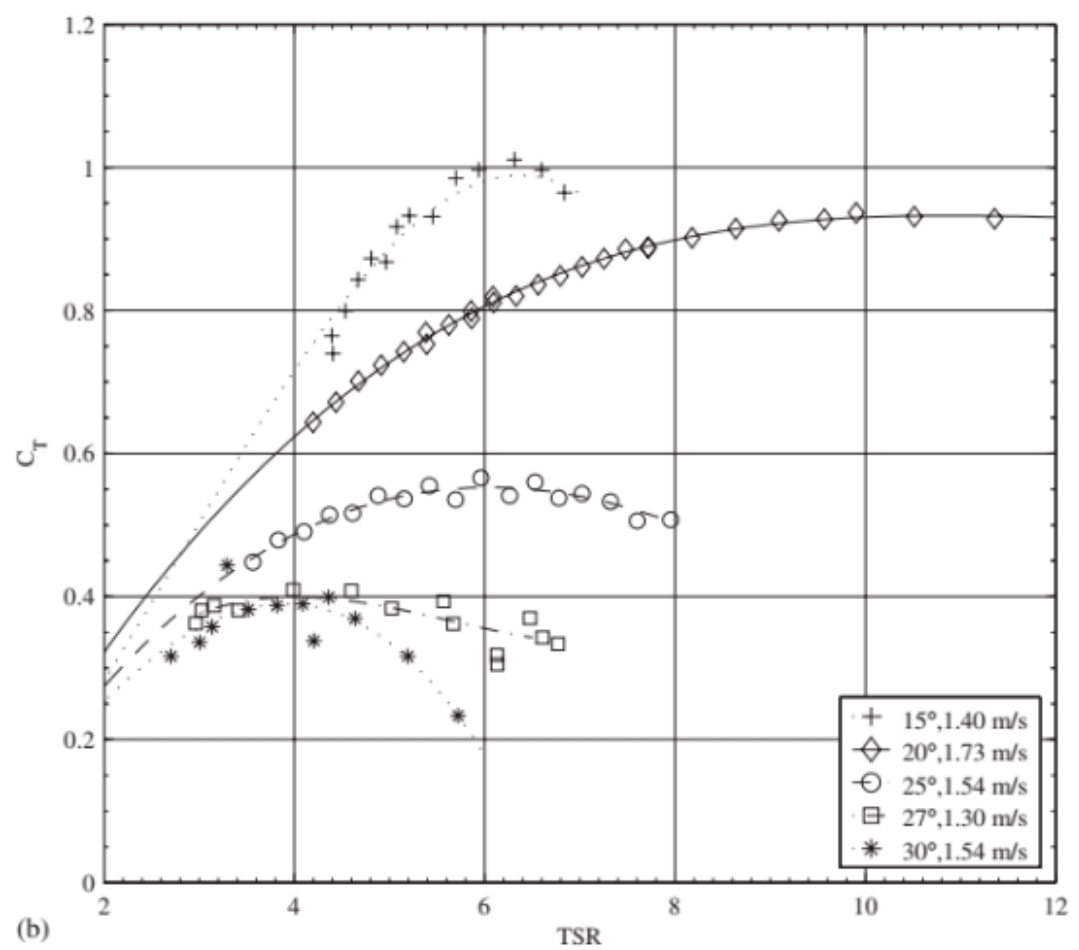

Source: (Bahaj, W.M.J., \& McCann, 2007) 
The results from the cavitation tunnel tests are shown in Figure 11 in which the pitch angle of $20^{\circ}$ presented higher values of power coefficient. For this reason, the simulations carried out on this work are made for this pitch angle.

In (Pinón et al., 2014) a single turbine was assessed experimentally to study the turbulence effects on the flow characteristics and performance. The turbine evaluated has a $700 \mathrm{~mm}$ diameter, based on a NACA63418 profile, studied in a TSR (ratio between tangential speed in the turbine pitch and the axial speed) range between 0 and 10. Figure 13 shows the experimental scheme used for the analysis. There is a torque sensor installed inside the hub, and the turbine structure is assembled in a base with a load cell to measure the thrust. Downstream of the turbine a Laser Doppler Velocimetry (LDV) system evaluated the wake velocity profile. The system is adjustable to cover a reasonable portion of the downstream length.

Figure 14 shows the results of power coefficient measurements. Near the peak of $\boldsymbol{C}_{\boldsymbol{P}}$ (power coefficient, defined at section 5.2.1) is seen that the increasing of the inlet turbulence intensity makes the curve less smooth. However, the global level of generated power is not affected. For the wake characteristics, the increasing of inlet turbulence level made faster the mixing between the wake and the remaining flow, which could improve the global efficiency of a turbine array, since the turbines could be put together more closely increasing the total number of turbines in the same area. The same authors have assessed two turbines axially aligned achieving the same results for wake mixing length decreasing with inlet turbulence levels increasing (Pinón et al., 2014B). 
Figure 13 - The Experimental scheme for evaluating the turbulence effects on flow through a tidal current turbine

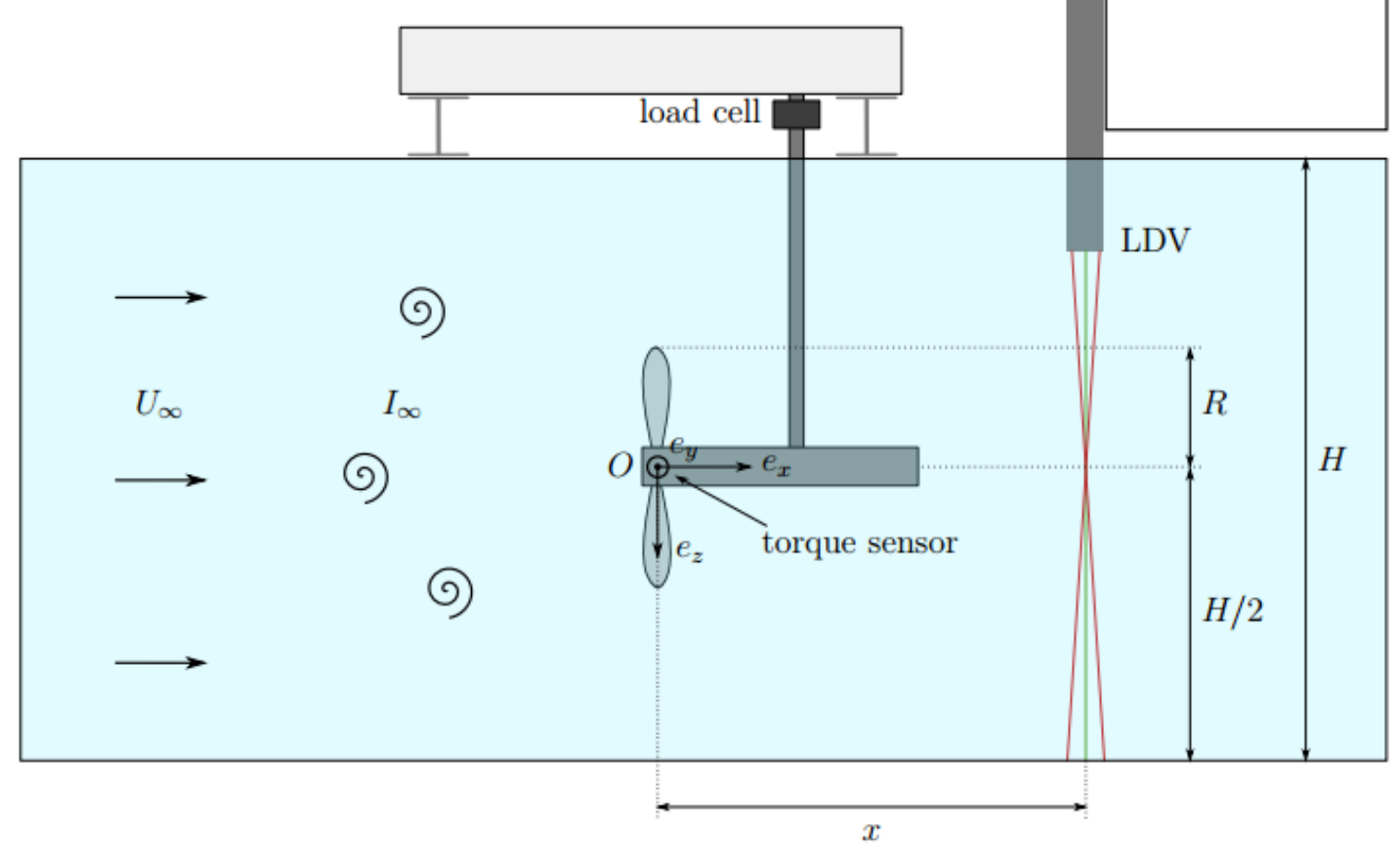

Source: (Pinón et al., 2014)

Figure 14 - Results from power (Top Figures) and thrust (Bottom Figures) measurements with different inlet turbulence intensities - 3\% on Left and $5 \%$ on Right
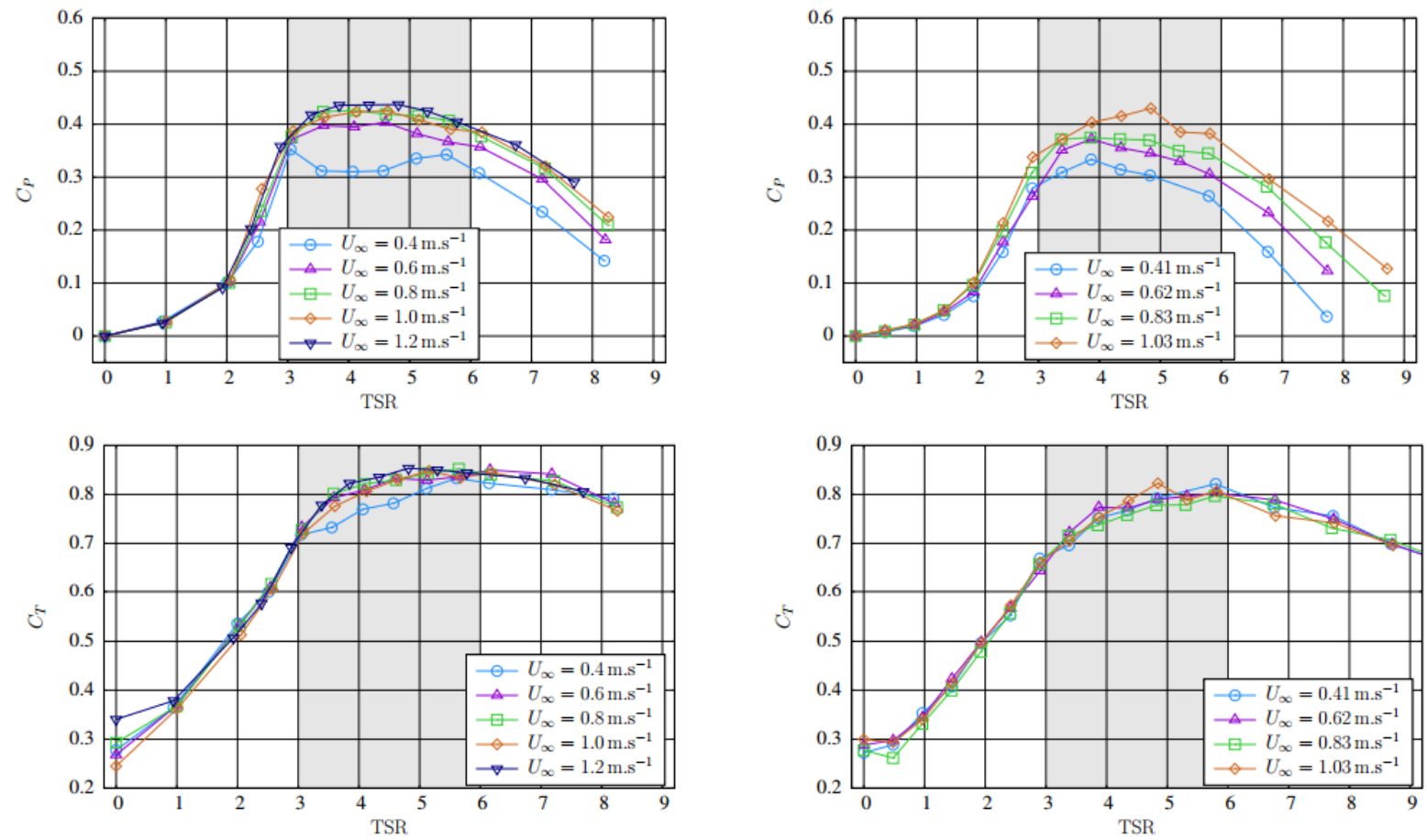

Source: (Pinón et al., 2014) 


\subsubsection{Computational Fluid Dynamics Analysis}

(Bir et al., 2011) performed a one-way Fluid Structure Analysis with the pressure data imported from CFD to the structural analysis. The authors used a $180^{\circ}$ model for a two-blade turbine, with the horizontal plane as symmetry plane. Effects due to free surface and seabed could not be analysed with such simplification in the model. The mesh consisted in approximately $3 \times 10^{6}$ elements with higher refinement close to the blades. With these results, the external shape of the blade was maintained, and the internal structural design was optimized following buckling and stress criterion.

(Lloyd et al., 2011) studied the turbine from (Bahaj, W.M.J., \& McCann, 2007) and employed a numerical simulation. The GGI technique was used with an open source CFD code (OpenFOAM) to analyse the hydrodynamic performance of the turbine. A transient analysis, using $k-\omega-S S T$ turbulence model with SIMPLE algorithm for couple pressure and velocity was used. The mesh consists in approximately $7.10^{5}$ hexahedral elements. The work did not present full convergence of the model and the domain length used for the analysis was shown to be short. The work underpredicted the power generated by the turbine.

(Afgan et al., 2012) compared two approaches for turbulence modelling in tidal current turbines. For code validation, a standard k- $\omega$-SST was used and then, a more robust LES code was implemented to make a comparison. The simulations were carried using the geometry from (Bahaj, W.M.J., \& McCann, 2007). A mesh sensitivity analysis was performed for the LES turbulence model, choosing a mesh with $7.4 \times 10^{6}$ volumes. The authors found that the differences in pressure distribution between blades pressure and suction sides lead to an underprediction of thrust and power coefficients. However, for TSR near the peak of $\boldsymbol{C}_{\boldsymbol{P}}$ values, both turbulence models achieved close results with the experimental analysis. The work has neglected the free surface effects, even on the transient analysis.

(Gebreslassie et al., 2012) performed a CFD analysis of a turbine modeled as momentum source, generating only lift and drag, with results provided by a Hybrid BEMT-CFD methodology to reduce computational cost. The authors performed a 3D simulation with the OpenFOAM CFD package. This CFD model was compared with an analytical proposal to model wake interactions trough factors based on linear 
actuator disk model. The main conclusion of the work is that a distance of 20 diameters between two turbines is sufficient for each turbine to achieve at least $91 \%$ of its individual power.

(Mason-Jones et al., 2012) the hydrodynamic performance of the turbine is assessed for a non-uniform profile flow at the inlet. The data from profiled flow was obtained from measurements made at site. The text is based on the hypothesis that the power coefficient is function of Reynolds number and TSR value, neglecting the influence of free surface effects. The CFD analysis was carried out with RANS equations with the Reynolds Stress transport turbulence model. A Moving Reference Frame (MFR) technique was applied to take the rotational effects in account,. The mesh was refined near the blade region to achieve a $\boldsymbol{y}^{+}$of 300 that has shown mesh convergence of the results. The domain size in the streamwise direction was 10 diameters in upstream direction and 40 diameters in the downstream direction. The CFD with a uniform profiled flow was validated with experimental measurements, showing a good agreement between numerical and test results for a TSR range between 5 and 6 . The conclusion of the work is that both power and thrust coefficients are independent from Reynolds number greater than $3 \times 10^{5}$, allowing experimental and numerical results based on test scale be scaled without corrections in Reynolds number once the inlet velocity profile is correctly represented.

(Pinón et al., 2012) used a particle method instead of the Finite Volume Method for domain discretization. The method uses a lagrangian description of the velocity field and, in this case, no turbulence or viscous effects was considered. The blades were modeled as vortex panels. The method had a good agreement for low TSR values, however, near to the peak of Power coefficient, the numerical results overpredicted the performance of the turbine. It occurs due to the misprediction of flow characteristics as separation that limits the lift generated in the turbine blades. However, for wake characterization, both quantitative and qualitative analysis have shown good results.

(Sharma et al., 2013) analysed the data from a site located in the United Kingdom, from an area sheltered from large surface waves and with currents in the order of $2.5 \mathrm{~m} / \mathrm{s}$ was analysed. The data consisted of velocities on the streamwise, transversal and vertical directions in time, providing realistic turbulence measurements. It was 
found high turbulence levels with approximately $12 \%$ of intensity with respect to the streamwise mean velocity, $9 \%$ to the transversal velocity and $7 \%$ to the vertical direction.

(Biskup et al., 2014) dealt with the problem of fluid structure interaction in tidal current turbines and highlight the high computational costs for a complete simulation within the full CFD analysis of the problem (including the rotor and the complete structural analysis with the required mesh refinement ensuring the correct calculation of stress gradients in the complex geometric transitions from the turbine blade). The authors proposed a multibody analysis of the problem instead of a full modelling of the structure. The results were fairly accurate, however, an experimental approach was still required for the complete validation of the problem.

(Blackmore et al., 2014) analysed the influence of varying inlet turbulence intensity on the wake characteristics of the turbine. The turbine was modeled as a momentum source and a LES method was used to model the turbulence, based on the Smagorinsky traditional approach. For initial condition, a pre-simulation using RANS equations was performed. They concluded that the turbulence intensity accelerates the wake recovery, improving the global performance of an array of turbines.

(Masters et al., 2015) compared a full-CFD approach with BEMT and the hybrid method BEM-CFD for analyse the flow through a tidal current turbine. The boundary layer due to the seabed shear effects was considered. For the lateral walls and free surface, a symmetry condition was applied simulating free slip walls. The domain size was chosen to ensure low blockage effects on the turbine. The same model was constructed for the BEM-CFD approach, with a mesh with approximately $3 \times 10^{6}$ elements. The simulations were carried out with the $k-\varepsilon$ turbulence model. The comparison between the results have shown a good agreement between the two results for power, thrust and even wake profile. A BEMT model was employed to calculate the power and thrust, revealing an underprediction of $5.4 \%$ in comparison with BEM-CFD. That paper also presented modelling of the coastal area with obstacles in the flow, now modelling the turbine as a porous disk in order to obtain the velocity distribution in the turbine region inlet. A full model considering the turbine geometry and the environment is still prohibitive, as far as computational costs are concerned. 
(Morris et al., 2016) performed a CFD analysis for turbines with two, three and four blades. The parameter of analysis is the Swirl number, $S_{\phi}$, which is the ratio between angular momentum flux and axial momentum flux, according to the equations ( 3.1 ), ( 3.2 ) and ( 3.3). For the CFD, they employed a domain with 10D for the upstream length and $30 \mathrm{D}$ for the downstream length (where $\mathrm{D}$ is the rotor diameter), with $2 \times 10^{6}$ elements composing the mesh. No free surface effects were considered. The researchers found a positive correlation between the number of blades (associated with the solidity of the rotor disk), and the swirl number. However, the results showed that the swirl number for tidal current turbines is low and presents a fast decay, which means that the increasing in the tangential velocities compared with axial velocities is small $a, d$ therefore the wake will mix with the flow faster

$$
\begin{gathered}
S_{\phi}=\frac{G_{\phi}}{G_{z} R} \\
G_{\phi}=\int_{0}^{R}\left(u_{\phi} r\right) \rho u_{z} \cdot 2 \cdot \pi \cdot r \cdot d r \\
G_{z}=\int_{0}^{R}\left(u_{z} \cdot \rho \cdot u_{z}+p \cdot\right) 2 \cdot \pi \cdot r \cdot d r
\end{gathered}
$$

Where:

$S_{\phi} \quad$ Is the Swirl number;

$G_{\phi} \quad$ Is the tangential momentum flux through the turbine disk;

$G_{z} \quad$ Is the axial momentum flux through the turbine disk;

$R \quad$ Is the turbine disk radius;

$u_{\phi} \quad$ Is the tangential velocity;

$u_{z} \quad$ Is the axial velocity.

(Tatum et al., 2016) considered the effects of surface gravity waves and varying profile flows in their study. The velocity profile was considered as a power from the height measured from the lower boundary. The turbine was modeled in its full scale. The work was conducted using the k-w-SST turbulence model, and the simulation was performed with the commercial code ANSYS CFX. The free surface was 
resolved by the Volume of Fluid (VOF) method, which required a high refinement of the mesh close to the free surface. The waves used in the simulation were modeled through potential wave theory for finite depths. The conclusion of the work is the high alternating stress caused due to gravity waves induced velocities. Both power and thrust have oscillated in the order of $10 \%$ of its mean value, which is supposed to cause electrical grid instabilities.

(Kulkarni et. al, 2016) studied the mesh dependency on results of steady-state simulations of the flow through a horizontal axis tidal current turbine. In the study, they used a full-scale turbine model, with a blade radius of $7.4 \mathrm{~m}$, based on a NACA0018 profile, reaching a mesh with 2440439 nodes.

(Zhang et al., 2017) compared the wake profiles at the inlet and wave characteristics measured in an experimental flume and compared with results obtained with a CFD analysis. The commercial code ANSYS Fluent 18.0 was used for the numerical analysis that was performed with RANS equations with $\mathrm{k}-\omega$-SST turbulence model. The mesh convergence study pointed out to a mesh with approximately $5 \times 10^{6}$ elements. The free surface was modeled as a free slip wall since the experiments did not present a significant head difference between upstream and downstream regions of the flow. The numerical analysis agreed with the results obtained from the experiments regarding thrust and torque coefficients. For the far-wake velocity profile measurements, CFD also presented good results also, validating the numerical method.

In Table 1 the works described above in this section are summarized highlighting the turbulence model, domain size, model used for free surface, the goals of the work and main results. 
Table 1 - Summary of CFD works on tidal current turbines

\begin{tabular}{|c|c|c|c|c|c|}
\hline Reference & $\begin{array}{c}\text { Turbulence } \\
\text { Model }\end{array}$ & $\begin{array}{l}\text { Domain } \\
\text { Size }\end{array}$ & Mesh & $\begin{array}{c}\text { Free Surface } \\
\text { Model }\end{array}$ & Simulation Objectives \& Results \\
\hline Bir et al. (2011) & $k-\omega-S S T$ & $\begin{array}{c}\text { Not } \\
\text { informed }\end{array}$ & $\begin{array}{c}\text { Not } \\
\text { informed }\end{array}$ & $\begin{array}{l}\text { No free } \\
\text { surface }\end{array}$ & Structural analysis and pressure distribution on blades. \\
\hline Lloyd et al. (2011) & $k-\omega-S S T$ & $\begin{array}{c}\text { Not } \\
\text { informed }\end{array}$ & $\begin{array}{l}7 \times 10^{5} \\
\text { elem. }\end{array}$ & $\begin{array}{l}\text { No free } \\
\text { surface }\end{array}$ & GGI analysis. The transient analysis did not converge. \\
\hline Afgan et al. (2012) & $\begin{array}{c}\mathrm{k}-\omega-S S T \& \\
\text { LES }\end{array}$ & $\begin{array}{c}\text { Not } \\
\text { informed }\end{array}$ & $\begin{array}{l}7.4 \times 10^{6} \\
\text { elem. }\end{array}$ & $\begin{array}{l}\text { No free } \\
\text { surface }\end{array}$ & $\begin{array}{l}\text { Turbulence model influence on results. They concluded } \\
\text { that k- } \omega \text {-SST is valid for } \boldsymbol{C}_{\boldsymbol{P}} \text { (power coefficient) } \\
\text { calculation, }\end{array}$ \\
\hline $\begin{array}{l}\text { Gebreslassie et al. } \\
(2012)\end{array}$ & LES & $\begin{array}{c}\boldsymbol{L}=51 \boldsymbol{D} \\
\boldsymbol{B}=25.3 \boldsymbol{D} \\
\boldsymbol{H}=10.3 \boldsymbol{D}\end{array}$ & $\begin{array}{c}\text { Not } \\
\text { informed }\end{array}$ & VOF Method & $\begin{array}{l}\text { Comparison of an analytical method for wake velocity } \\
\text { modelling with CFD analysis with full blade resolved. } \\
\text { The analytical results were close to the CFD results. }\end{array}$ \\
\hline $\begin{array}{l}\text { Mason-Jones et al. } \\
(2012)\end{array}$ & $\begin{array}{l}\text { Reynolds } \\
\text { Stress } \\
\text { Transport }\end{array}$ & $\begin{array}{l}L=50 D \\
B=5 D \\
H=5 D\end{array}$ & $\begin{array}{c}9.7 \times 10^{5} \\
\text { MRF }\end{array}$ & Free-Slip wall & $\begin{array}{l}\text { Effects of inlet varying profiled flow in the performance } \\
\text { characteristics and analyse the } \boldsymbol{R}_{E} \text { dependence on } \\
\text { Results. They found that above the results are } \\
\text { independent for } \boldsymbol{R}_{E}>3 \times 10^{5} \text {. }\end{array}$ \\
\hline Pinón et al. (2012) & $\begin{array}{l}\text { No } \\
\text { turbulence } \\
\text { effects }\end{array}$ & $\begin{array}{c}\text { Not } \\
\text { informed }\end{array}$ & $\begin{array}{c}\text { Not } \\
\text { informed }\end{array}$ & $\begin{array}{l}\text { No free } \\
\text { surface }\end{array}$ & $\begin{array}{l}\text { Evaluate the tidal current turbine performance without } \\
\text { viscosity and turbulence. The analysis overpredicted the } \\
\text { generated power even with a good agreement } \\
\text { concerning the wake profile. }\end{array}$ \\
\hline
\end{tabular}




\begin{tabular}{|c|c|c|c|c|c|}
\hline Reference & $\begin{array}{l}\text { Turbulence } \\
\text { Model }\end{array}$ & $\begin{array}{l}\text { Domain } \\
\text { Size }\end{array}$ & Mesh & $\begin{array}{l}\text { Free Surface } \\
\text { Model }\end{array}$ & Simulation Objectives \& Results \\
\hline $\begin{array}{l}\text { Sharma et al. } \\
\text { (2013) }\end{array}$ & Not informed & $\begin{array}{c}\text { Not } \\
\text { informed }\end{array}$ & $\begin{array}{c}\text { Not } \\
\text { informed }\end{array}$ & $\begin{array}{l}\text { No free } \\
\text { surface }\end{array}$ & $\begin{array}{l}\text { Experimental assessment of the turbulent intensity at } \\
\text { the site. Realistic turbulence intensity levels for model } \\
\text { calibration with } 12 \% \text { of intensity in the streamwise } \\
\text { direction, } 9 \% \text { in the transversal direction and } 7 \% \text { in the } \\
\text { vertical direction. }\end{array}$ \\
\hline $\begin{array}{l}\text { Biskup et al. } \\
\text { (2014) }\end{array}$ & $k-\omega-S S T$ & $\begin{array}{c}\text { Not } \\
\text { informed }\end{array}$ & $\begin{array}{c}\text { Not } \\
\text { informed }\end{array}$ & $\begin{array}{l}\text { No free } \\
\text { surface }\end{array}$ & $\begin{array}{l}\text { The authors validated a multibody approach for fluid- } \\
\text { structure interaction instead of full structural analysis of } \\
\text { turbine blades. }\end{array}$ \\
\hline $\begin{array}{l}\text { Blackmore et al. } \\
(2014)\end{array}$ & LES & $\begin{array}{l}\text { Depends } \\
\text { on grid } \\
\text { inlet }\end{array}$ & $\begin{array}{c}\text { Not } \\
\text { informed }\end{array}$ & Free-Slip wall & $\begin{array}{l}\text { Evaluate the effects of inlet turbulence intensity on the } \\
\text { wake profile. The results are similar to these found by } \\
\text { other authors in which the increase in turbulence levels } \\
\text { decreases the wake mixing length. }\end{array}$ \\
\hline $\begin{array}{l}\text { Masters et al. } \\
\text { (2015) }\end{array}$ & $k-\varepsilon$ & $\begin{array}{c}\text { Not } \\
\text { informed }\end{array}$ & $3 \times 10^{6}$ & Free-Slip wall & $\begin{array}{l}\text { Compares the results for full-blade resolved CFD, BEM- } \\
\text { CFD and BEMT. All methodologies presented a good } \\
\text { agreement with experimental results. BEMT } \\
\text { underpredicts the peak power coefficient. }\end{array}$ \\
\hline Morris et al. (2016) & $\begin{array}{l}\text { Reynolds } \\
\text { Stress } \\
\text { Transport }\end{array}$ & $\begin{array}{l}L=40 D \\
B=5 D \\
H=5 D\end{array}$ & $2 \times 10^{6}$ & $\begin{array}{l}\text { No free } \\
\text { surface }\end{array}$ & $\begin{array}{l}\text { Evaluate the turbine swirl and its relation to the number } \\
\text { of blades. The results pointed in a low swirl number for } \\
\text { this kind of turbine and the dependence between blades } \\
\text { number and swirl number. }\end{array}$ \\
\hline
\end{tabular}




\begin{tabular}{|c|c|c|c|c|c|}
\hline Reference & $\begin{array}{c}\text { Turbulence } \\
\text { Model }\end{array}$ & $\begin{array}{l}\text { Domain } \\
\text { Size }\end{array}$ & Mesh & $\begin{array}{l}\text { Free Surface } \\
\text { Model }\end{array}$ & Simulation Objectives \& Results \\
\hline Tatum et al. (2016) & $k-\omega-S S T$ & $\begin{array}{c}\text { Not } \\
\text { informed }\end{array}$ & $2.8 \times 10^{6}$ & VOF & $\begin{array}{l}\text { Non-uniform inlet velocity profile and free surface waves } \\
\text { were introduced in the boundary conditions to evaluate } \\
\text { their results on the hydrodynamic performance. } \\
\text { Oscillations in power and thrust were found for the flow } \\
\text { with the effect of gravity waves. }\end{array}$ \\
\hline $\begin{array}{l}\text { Kulkarni et al. } \\
\text { (2016) }\end{array}$ & $\begin{array}{l}k-\omega-S S T \\
k-\varepsilon R N G\end{array}$ & $\begin{array}{c}\text { Not } \\
\text { informed }\end{array}$ & $2.4 \times 10^{5}$ & $\begin{array}{l}\text { No free } \\
\text { surface }\end{array}$ & $\begin{array}{l}\text { The authors performed a mesh convergence analysis of } \\
\text { two different two equations turbulence models. They } \\
\text { concluded that k- } \omega \text {-SST provides a better numerical } \\
\text { prediction of the hydrodynamic performance. }\end{array}$ \\
\hline Zhang et al. (2017) & $k-\omega-S S T$ & $\begin{array}{c}L=211 D \\
B=4.4 D \\
H=2 D\end{array}$ & $5 \times 10^{6}$ & Free-Slip wall & $\begin{array}{l}\text { The work analysed the wake from tidal current turbines } \\
\text { with full resolved blades. The wake profile has deviated } \\
\text { from experimental predictions. The authors justify it due } \\
\text { to the limitations of the turbulence model. }\end{array}$ \\
\hline
\end{tabular}

Where $\boldsymbol{L}$ is the domain Length, $\boldsymbol{B}$, the domain width, $\boldsymbol{H}$ the domain Height and $\mathrm{D}$ is the turbine Diameter; 


\subsubsection{Analytical models}

(Garret \& Cummins, 2007) presented an actuator disk model to take into account the effects of blockage. The turbine was fully confined and with no influence of free surface effects. The authors showed that the power coefficient overcomes the Betz Limit (the theoretical limit of 16/27 of the power coefficient in the case of an open flow) by a factor equal to $(1-B)^{-2}$ in which the power coefficient tends to infinite as the Blockage Ratio tends to zero. However, the model will lead to an infinite pressure drop, which does not occur in real situations.

(Houlsby et al., 2007) further developed the model for the actuator disk model with different boundary conditions. A first model considers the actuator disk in an open flow, conducing to the Betz Limit. A second model confined by walls and by constant pressure are included evaluating the effects of blockage in the hydrodynamic performance. The last model considers an open channel flow, in which in the lower boundary, the flow is defined by a rigid wall and in the upper boundary the as a constant pressure wall.

(Peiró et al., 2007) proposed a different scheme from (Houlsby et al., 2007) to model an open channel actuator disk model equations. They proposed a potential model for the interaction between the free surface with a turbine array. The model consists of introducing the linear wave potential in the free surface modelling the turbines as porous barriers.

(Masters et al., 2011) used the Blade Element Momentum Theory (BEMT), including tip and hub corrections factors to perform a blade optimization. The results were compared those obtained with a commercial code for tidal current turbines and the original Lifting Line Theory. The results showed a good agreement.

(Galloway et al., 2011) proposed a modified version of the BEMT including the velocity field generated by waves in the inlet velocity, carrying out a traditional method to calculate thrust and torque coefficients. The authors have performed a transient analysis which concluded that power fluctuations could represent an issue for the power conversion mechanisms even without significant changes in the total amount of generated power. Besides, for the turbine structure, the cyclical loads 
could lead to fatigue failure. Thus, this load must be taken into account during the turbine design.

(Estafahani \& Karbasian, 2012) the authors highlight the similarity between the physics of axial tidal current wind turbines. The formulation is based on the Blade Element Momentum Theory. The shape of the blades is optimized pursuing the maximum ratio between lift and drag for each section. They do not refer to any known optimization technique based on an objective function subjected to restrictions.

\subsubsection{Tidal farm and wind farm optimization methods}

The distribution of turbines in a tidal or wind farm may follow similar position that the obtained from the optimization methods.

(Piggott et al., 2014A) approached the optimization problem is based on a twodimensional CFD approach, varying the number of turbines and the channel shape. The turbine is modeled as a friction constructed from bump functions. The objective function to be optimized is the total averaged power which is proportional to the velocity cubed. The optimization algorithm is based on gradients.

(Piggott et al., 2014B) presented a comparison between the methods for optimizing a tidal-current farm for maximum power generation. The importance of this work is that an increase of the model fidelity could lead to unfeasible computational costs. For example, fully resolved blade simulation which produces accurate results are expensive even for a single turbine simulation.

(Forinash \& DuPont, 2016) proposed an optimization algorithm for offshore wind power plants. They introduced the total cost of the wind turbine farm as the objective function to optimize the difference between the total cost and the profit from the energy sold to the electricity grid. In order to determine the power generated by each turbine, they used an analytical model with wake propagation. The number of turbines and their position were the design variables.

(Stansby \& Stallard, 2016) employed an experimental analysis of a tidal current turbine to evaluate the velocity profile at the wake. They provided an empirical formulation for the mean wake velocity, width and velocity profile. 
Hence, the power is proportional to the third power of the axial velocity, which is considered the velocity distribution along the rotor radius. The objective function is the total power generated by the turbine array, and the algorithm is based on the gradient of the power function. The authors highlighted that the blockage effects were estimated, but still require a further improvement for a better prediction.

The analysed papers showed that the computationally feasible methodologies for optimizing tidal current turbine and wind turbine farm power generation is based on the actuator disk models, even when CFD was used. Hence, the agreement between the analytical models for a single turbine with the real velocity profiles and the power generation is mandatory for the accuracy of the optimization models.

\subsection{Open channel models}

(Ferziger \& Peric, 2002) divided the methods for free surface computational analysis in surface capturing and in surface tracking. The first method includes the VOF approach, in which the position of the free surface position is estimated based on the proportion of water and air on each cell. In the surface tracking method, it is necessary to obtain a height function for the interface. The authors proposed a function based on the kinematic condition for free surface waves based mainly on the flow direction that must remain tangent to the free surface to ensure that no mass flux occurs through the free surface streamline.

(Battaglia et al., 2004) analysed the free surface flow with the Finite Element Method approach. A lagrangian scheme is used for the free surface, tracking its movement and updating the mesh in every iteration. The boundary conditions at the free surface are forced atmospheric pressure on free surface and no tangential stresses in the free surface.

For mesh updating, they considered that the mesh is contained in an elastic body and the mesh moves according to the body displacement field due to its strain. A smoothing algorithm is introduced for the free surface to adjust diffusivity. The paper validated the methodology with $2 \mathrm{D}$ and $3 \mathrm{D}$ viscous sloshing models, and both frequency and viscous damping were accurately predicted. 
(Reichl et al., 2005) investigated the flow around a cylinder close to a free surface employing a CFD with a VOF approach in a transient analysis. During the analysis, it was found that for a Froude number close to 0.4 the non-linear phenomena of wave braking started to appear. It is proposed a local Froude number analysis considering the mean velocity between the obstacle and the free surface. The goal of the analysis is studying the Strouhal number behaviour with the free surface proximity

(Mattavo \& Assi, 2017) proposed an alternative methodology for free surface treatment which was tested with the flow around a circular cylinder. Results were compared with the obtained with VOF methodology. This model is based on potential energy conservation substituting the gravity potential energy by the elastic potential energy obtained through an elastic wall from a Fluid-Structure Interaction approach between the fluid domain and a solid domain containing an orthotropic body model. 


\section{Chapter 4. Theoretical Fundamentals}

This chapter presents the theoretical fundamentals that are necessary to perform a CFD analysis of an axial tidal current turbine and model the elastic free surface by coupling an orthotropic elastic body.

The CFD methodology is based on Finite Volume Method and solves the Reynolds Averaged Navier-Stokes equations. Therefore, it is presented a review of the equations for a viscous, incompressible and turbulent fluid flow. Besides, a brief discussion about turbulence models is performed, since the correct chose of the methodology is fundamental for an accurate simulation.

The potential fluid flow theory is introduced since a theoretical analysis of potential fluid flow through a circular cylinder close to a free surface is performed using an elastic-free surface boundary condition. Besides, the gravity wave theory lies in the potential fluid flow theory. Therefore, the introduction of its basics concepts is mandatory.

Still, on fluid mechanics, the integral equations for fluid mechanics are introduced. There is a classic approach for axial turbines based on the one-dimension fluid flow and using the balance between mass, momentum, and energy to estimate the power and thrust generated in a turbine. For this balance, the computation of the integral quantities in several sections of the fluid flow is necessary, justifying the presentation of such equations.

The solid mechanics review is based on Theory of Elasticity applied for orthotropic solid bodies. The free surface is simulated as a free-slip elastic wall. In order to provide to this wall a stiffness independent of the position is necessary that the solid not transmit shear stresses. Hence, the orthotropic solid is required instead of the classical isotropic solid modeled originally in the classical Theory of Elasticity. The section 4.3 includes the basic equations for Theory of Elasticity, the definition of Airy's stress function and the derivation of the differential equation for an orthotropic solid with a negligible shear modulus. 
Finally, a finite volume method and finite element method review are included. In this section, only the basics of both theories are presented, and the details of the elements used in the simulations are provided in the Attachment.

\subsection{Basics equations of Fluid Dynamics}

The flow through an axial tidal current turbine is commonly modeled as a viscous, incompressible and fully turbulent flow. Some authors use potential methodologies, which considers the viscosity within theoretical assumptions such as the Kutta Condition that states the fluid velocity is finite in an airfoil trailing edge, and then distributes vortex and sources in the blade surfaces. However, this work is based on viscous, turbulent and incompressible fluid flow.

An incompressible and viscous fluid flow is governed, for a Newtonian fluid, by the Navier-Stokes equations, and by the mass conservation equation. The Navier-Stokes equations represent the conservation of the momentum, in which the left hand of the equation represents the acceleration of a fluid particle and the right hand represents the surface and body forces actuating in this fluid particle. The equation is written below:

$$
\frac{\partial}{\partial t}\left(u_{i}\right)+\frac{\partial}{\partial x_{j}}\left(u_{i} u_{j}\right)=-\frac{1}{\rho} \frac{\partial p}{\partial x_{i}}+\frac{\partial}{\partial x_{j}}\left(v \frac{\partial u_{i}}{\partial x_{j}}\right)+g_{i}
$$

Where:

$\begin{array}{ll}u_{i} & \text { Is the velocity vector; } \\ \rho & \text { Is the fluid density; } \\ p & \text { Is the fluid pressure; } \\ v & \text { Is the fluid kinematic viscosity; } \\ g_{i} & \text { Is the gravity acceleration. }\end{array}$

The mass conservation equation for a continuous media is written below:

$$
\frac{\partial}{\partial t}(\rho)+\frac{\partial}{\partial x_{i}}\left(\rho u_{i}\right)=0
$$


If the fluid is incompressible, the density is constant along the time, which means that the volume of a fluid particle must remain constant during the flow, which is written by the following equation:

$$
\frac{\partial}{\partial x_{i}}\left(u_{i}\right)=0
$$

The presented equations apply for both laminar and turbulent flows. However, due to the nature of a turbulent flow which is characterized by an aleatory motion of the fluid particles. A high mesh is required for tracking their position, being unfeasible for most of the industrial fluid flow analysis. A common approach is to model a turbulent flow by their main quantities, and in this approach, a variable is divided into two parts, as follows in the equation below:

$$
\varphi=\bar{\varphi}+\varphi^{\prime}
$$

Where:

$$
\begin{array}{ll}
\varphi & \text { Is a generic quantity; } \\
\bar{\varphi} & \text { Is the mean of this generic quantity; } \\
\varphi^{\prime} & \text { Is the fluctuation of this generic quantity; }
\end{array}
$$

As property of the division of a generic quantity in a mean and a fluctuation parts, follows that both means of the fluctuation part and fluctuation of a mean part are equal to zero, which are represented by the following equations:

$$
\begin{aligned}
& (\bar{\varphi})^{\prime}=0 \\
& \overline{\left(\varphi^{\prime}\right)}=0
\end{aligned}
$$

Applying the division of the velocity and pressure in the Navier-Stokes equations and the mass conservation equation for an incompressible fluid flow and by taking the mean of both equations is derived the Reynolds Averaged Navier Stokes (RANS) equations which are written by:

$$
\begin{gathered}
\frac{\partial \bar{u}_{i}}{\partial t}+\frac{\partial\left(\bar{u}_{i} \bar{u}_{j}\right)}{\partial x_{j}}=-\frac{1}{\rho} \frac{\partial \overline{\mathrm{p}}}{\partial x_{i}}+g_{i}+\frac{\partial}{\partial x_{j}}\left(v \frac{\partial \bar{u}_{i}}{\partial x_{j}}-\overline{\mathrm{u}_{\imath}^{\prime} \mathrm{u}_{j}^{\prime}}\right) \\
\frac{\partial \bar{u}_{i}}{\partial x_{i}}=0
\end{gathered}
$$


Where:

$\bar{u}_{i} \quad$ Is the mean velocity vector;

$\overline{\mathrm{p}} \quad$ Is the mean fluid pressure;

$-\overline{\mathrm{u}_{\imath}^{\prime} \mathrm{u}_{\jmath}^{\prime}} \quad$ Is the Reynolds stress tensor.

The RANS model brings four equations (three equations from the mean velocity vector and one equation for mean pressure) to solve ten variables (three velocity components, mean pressure and six components from the Reynolds stress tensor). Hence, an additional hypothesis and equations are necessary to close the problem. In the next section, it is introduced some hypothesis as the turbulent viscosity, and some models involving the transport of turbulent kinetic energy are used.

Hitherto it was presented only the differential equations used for turbulent, viscous and incompressible fluid flow. However, for some analysis, an integral formulation, computing mean values of the flow is necessary too. Using the Reynolds transport equations, which is presented in (Munson, Young, \& Okiishi, 2002, pp. 163-170), a balance of an intensive scalar quantity $q$ is performed in a control volume according to the following equation:

$$
\frac{D}{D t} \iiint_{\forall_{C}} \rho q d \forall=\frac{\partial}{\partial t} \iiint_{\forall_{C}} \rho q d \forall+\iint_{S_{C}} \rho q(\vec{v} \cdot \vec{n}) d S
$$

Where:

$\forall_{C} \quad$ Is the control volume;

$S_{c} \quad$ Is the control surface (boundaries from the control volume);

$q \quad$ Is an intensive scalar quantity.

This equation is usually applied to mass, momentum, angular momentum and energy. For mass the equation becomes:

$$
\frac{D}{D t} \iiint_{\forall_{C}} \rho d \forall=\frac{\partial}{\partial t} \iiint_{\forall_{C}} \rho d \forall+\iint_{S_{C}} \rho(\vec{v} \cdot \vec{n}) d S
$$


Considering a fixed control volume and an incompressible flow, this equation is simplified to:

$$
\iint_{S_{c}} \rho(\vec{v} \cdot \vec{n}) d S=0
$$

Applying the equation ( 4.8 ) to the velocity vector:

$$
\frac{D}{D t} \iiint_{\forall_{C}} \rho \vec{v} d \forall=\frac{\partial}{\partial t} \iiint_{\forall_{C}} \rho \vec{v} d \forall+\iint_{S_{C}} \rho \vec{v}(\vec{v} \cdot \vec{n}) d S
$$

Considering a fixed control volume and an incompressible flow, this equation is simplified to:

$$
\rho \iint_{S_{c}} \vec{v}(\vec{v} \cdot \vec{n}) d S=\sum \vec{F}_{\text {ext }}
$$

Where:

$\sum \vec{F}_{\text {ext }} \quad$ Is the sum of external forces acting in the control volume.

Applying the equation ( 4.8 ) to the angular momentum.

$$
\frac{D}{D t} \iiint_{\forall_{C}} \rho(\vec{r} \times \vec{v}) d \forall=\frac{\partial}{\partial t} \iiint_{\forall_{C}} \rho(\vec{r} \times \vec{v}) d \forall+\iint_{S_{C}} \rho(\vec{r} \times \vec{v})(\vec{v} \cdot \vec{n}) d S
$$

Considering a fixed control volume and an incompressible flow, this equation is simplified to:

$$
\rho \iint_{S_{c}}(\vec{r} \times \vec{v})(\vec{v} \cdot \vec{n}) d S=\sum \vec{M}_{e x t}
$$

Where:

$\sum \vec{M}_{\text {ext }} \quad$ Is the sum of external moments acting in the control volume. 
For Blade Element Momentum Theory, the velocity vector is divided into two main components. The first component, with the streamwise direction and the second component with tangential direction. The radial component is neglected in such analysis. In this model, the conservation of angular momentum is performed between the inlet and outlet sections, using only the painted control volume shown in Figure 15. Applying the equation (4.15) in this domain is obtained the rotating annular stream tube angular momentum conservation equation:

$$
\frac{d M_{Z}}{d r}=2 \pi \cdot \rho \cdot \Delta \omega \cdot U \cdot r^{3}
$$

Where:
$M_{Z} \quad$ Is the moment in the flow direction axis;
$r \quad$ Is the radius of the analyzed portion of the rotating annulus;
$\Delta \omega \quad$ Is the difference of angular speed between the inlet and outlet.

The integral conservation of energy is not presented in this text since the Bernoulli's equation with losses is applied instead of the second law of thermodynamics. The Bernoulli's equation between a point "i" representing the inlet and a point "o" representing the outlet becomes:

$$
h_{i}+\frac{u_{i}^{2}}{2 g}+\frac{p_{i}}{\rho g}=h_{o}+\frac{u_{o}^{2}}{2 g}+\frac{p_{o}}{\rho g}+\frac{F_{\text {ext }}}{\rho g A}
$$

Where:

$\begin{array}{cl}h & \text { Is the static head; } \\ \frac{F_{\text {ext }}}{\rho g A} & \text { Is the losses between inlet and outlet due to external forces. }\end{array}$

Figure 15 - Domain with one inlet and one outlet aligned to flow direction

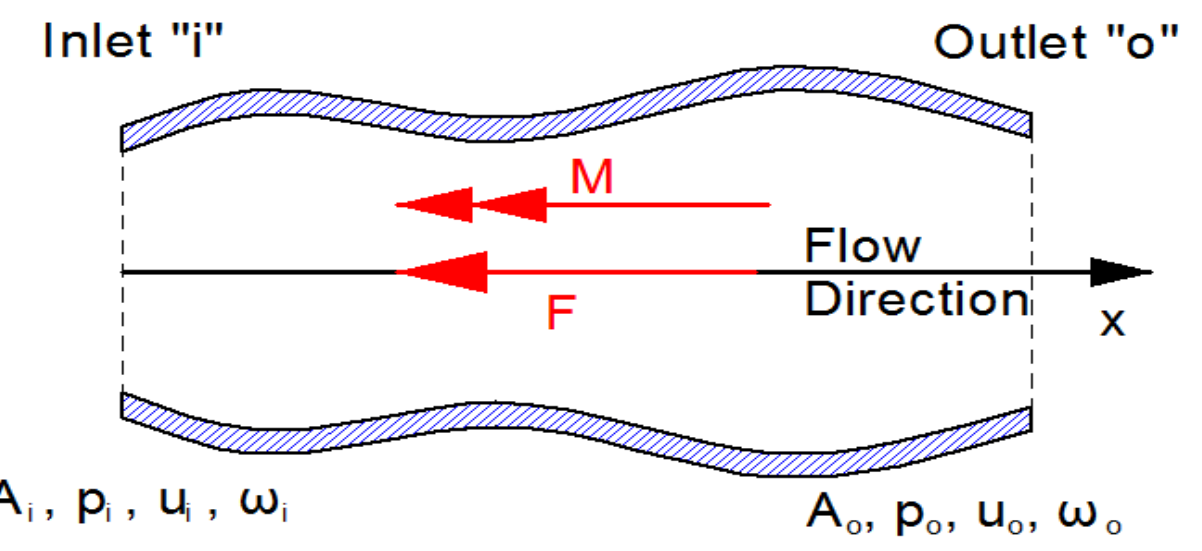




\subsubsection{Turbulence Models}

In the RANS model, a term related to the velocity vector fluctuation appears in the diffusive term from the equation. This term is called Reynolds stress tensor and is responsible for the momentum diffusion in the flow. In turbulent flows, for example, in regions far from the boundary layer, this term is more representative than the viscous shear stress term, which is presented in Figure 16, comparing the viscous and turbulent shear stresses in function of the distance from the wall. Hence, its modelling is necessary to guarantee the accuracy of the turbulent flow analysis.

There are several turbulence modelling methods. The most complete, Direct Navier Stokes Simulation (DNS), is computationally expensive since it simulates the unsteady Navier-Stokes equation without the division between mean and fluctuation components. That requires such domain and time step discretization that becomes unfeasible most of industrial flows analysis by such method. However, using the RANS model, there are some methods for calculating the Reynolds Stress Tensor. Moreover, in some of these methods, such as $\boldsymbol{k}-\boldsymbol{\varepsilon}$ and $\boldsymbol{k}$ - $\boldsymbol{\omega}$ - SST uses the turbulent viscosity hypothesis. It assumes that the turbulent shear stress is proportional to the velocity component as happens for the viscous shear stress. The turbulent viscosity hypothesis is represented by the following equation.

$$
-\overline{\mathrm{u}_{\imath}^{\prime} \mathrm{u}_{\jmath}^{\prime}}=v_{T} \frac{\partial \bar{u}_{i}}{\partial x_{j}}
$$

Figure 16 - Profiles of the fractional contributions of the viscous and Reynolds stresses, from DNS data, with dashed lines representing $R e=5600$ and solid lines $R e=13750$

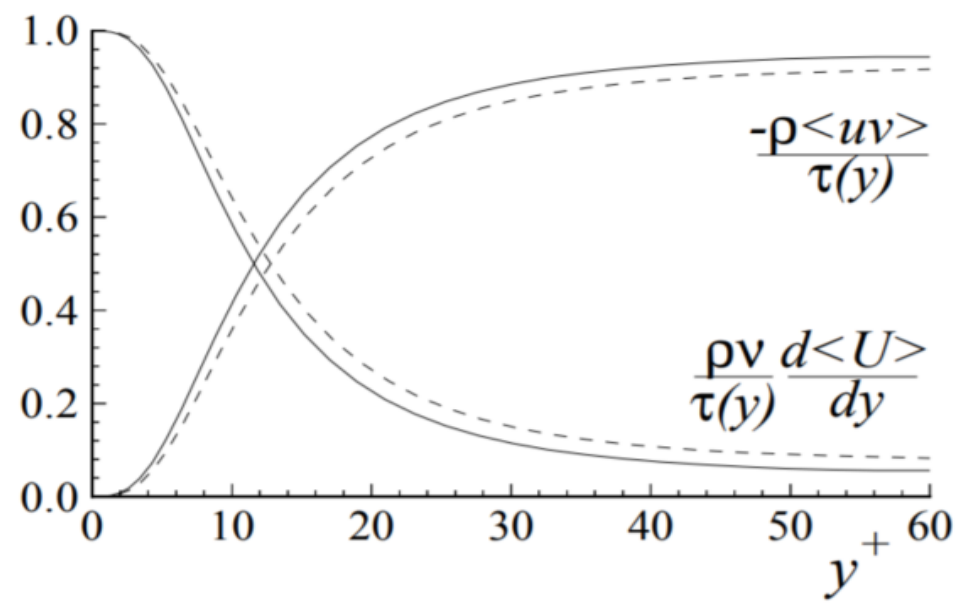

Source: (Kim \& Moser, 1987) 
The problem for solving the Reynolds Stresses becomes simpler since it originally has six components and now, according to this hypothesis, it is simplified to only one variable. However, even that such assumption leads to accurate results, this hypothesis is not physically consistent, since the turbulent shear stress proportional to the velocity gradient is a particular case of turbulent flow. Besides that, since that one more variable, the turbulent viscosity must be determined, at least one more equation needs to be derived to close this mathematical model.

The section 3.1.2 highlighted the turbulent models used by other authors. Is clearly seen that most of the works used the $\boldsymbol{k}$ - $\boldsymbol{\omega}$ - SST Model, which calculates the turbulent viscosity from the turbulent kinetic energy, $k$, and from the turbulent rotational kinetic energy, $\boldsymbol{\omega}$.

From the definition of the Reynolds stress tensor, it is defined by the turbulent kinetic energy, $k$, which is invariant and hence independent from the coordinate system.

$$
k=\frac{\overline{\mathrm{u}_{l}^{\prime} \mathrm{u}_{l}^{\prime}}}{2}
$$

The dissipation of turbulent kinetic energy is provided by the molecular viscosity. In this text, the complete transport equation for turbulent kinetic energy is not analyzed. However, it is found in (Wilcox, 1994, p. 74). The total amount of turbulent kinetic energy is related to production, $\mathcal{P}$, and dissipation, $\varepsilon$, of turbulence. Both terms of production and dissipation of turbulent kinetic energy are presented below:

$$
\begin{gathered}
\mathcal{P}=-\overline{\mathrm{u}_{i}^{\prime} \mathrm{u}_{j}^{\prime}} \frac{\partial \bar{u}_{i}}{\partial x_{j}} \\
\varepsilon=v \overline{\left(\frac{\partial \mathrm{u}_{i}^{\prime}}{\partial x_{j}} \frac{\partial \mathrm{u}_{i}^{\prime}}{\partial x_{j}}\right)}
\end{gathered}
$$

From this equation, is seen that the production is related to the total amount of Reynolds shear stress and the mean velocity gradient, while the dissipation is related to the gradients of velocity fluctuations. Besides, according to the equation ( 4.18 ), the turbulent diffusion increases with the turbulence intensity, which are related to the balance between production and dissipation. Therefore, there is fundamental to model those quantities with accuracy. 
The $\boldsymbol{k}$ - $\boldsymbol{\varepsilon}$ Model developed originally by (Jones \& Launder, 1972) for example, is a two-equation model in which there is a transport equation for the turbulent kinetic energy and other for turbulent kinetic energy dissipation. In (Pope S. , 2000, pp. 373383) is discussed that there is no diffusion of the turbulent kinetic energy near to wall region. It is highlighted the dependency from the model constants adjustment for each analysed kind of flow. However, the model behaviour is accurate in regions far from the boundary layer.

The $\boldsymbol{k}$ - $\omega$ model computes the turbulent viscosity from turbulent kinetic energy and turbulent rotational kinetic energy. This model is known by being superior to the $\boldsymbol{k}$ - $\boldsymbol{\varepsilon}$ near to the wall (Pope S. , 2000, p. 384), specially for non-homogeneous turbulent flows. However, for regions far from the boundary layer the $\boldsymbol{k}$ - $\boldsymbol{\varepsilon}$ Model still more accurate.

Further, a model which blends both $\boldsymbol{k}$ - $\boldsymbol{\varepsilon}$ and $\boldsymbol{k}$ - $\omega$, depending on the analysed region of the flow was developed by (Menter, 1992). This model is the so-called $\boldsymbol{k}$ - $\boldsymbol{\omega}$ - SST. A blending function is applied and it is dependent from the distance to the wall. With this treatment, the model behaves like $\boldsymbol{k}$ - $\omega$ near to the wall and $\boldsymbol{k}$ - $\boldsymbol{\varepsilon}$ at the freestream. According to this model, the turbulent viscosity is given by:

$$
v_{t}=\frac{a_{1} k}{\max \left(a_{1} \omega, S F_{2}\right)}
$$

Where:

$a_{1} \quad$ Is a constant;

$S \quad$ Is the mean vorticity magnitude;

$F_{2} \quad$ Is a function which depends of the distance to the wall.

The complete equations for this model are found in the Attachment B.

Even this model being representative in both near-wall and in free-stream with respect to the turbulence modelling, for an accurate boundary-layer representation and hence the calculation of wall shear stresses and flow separation, for example, is necessary a high domain discretization near to the wall. Hence, in some flow regions with higher velocities, a small element size is required. However, it is not feasible for some industrial problems, since the computational time increases with the number of elements. 
In order to solve this problem, some commercial CFD codes, such as the ANSYS CFX® which applies scalable wall functions based on (Launder \& Spalding, 1974). The complete formulation is presented in the Attachment $C$. In this context, it is presented in the Figure 17 a typical profile for a turbulent boundary layer in a flat plate. There is three different regions. The first one, called sublayer have the predominance of molecular viscosity effects. The next region is known as Log-Layer and comprehends a $y^{+}$between 10 and $10^{3}$ approximately. The last region is known as defect layer and represents the transition between the boundary layer and the free stream. Usually, it is used dimensionless distances to the wall according to the following equations:

$$
\begin{gathered}
\tau_{W}=\left.\rho v\left(\frac{d \bar{u}}{d y}\right)\right|_{y=0} \\
u_{\tau} \equiv \sqrt{\frac{\tau_{W}}{\rho}} \\
y^{+}=\frac{u_{\tau} \cdot y}{v}
\end{gathered}
$$

Where:

$$
\begin{array}{ll}
\tau_{W} & \text { Is the wall viscous shear stress; } \\
u_{\tau} & \text { Is the reduced velocity; } \\
y^{+} & \text {Is the dimensionless wall distance. }
\end{array}
$$

Figure 17 - Typical velocity profile for a turbulent boundary layer $U^{+}$

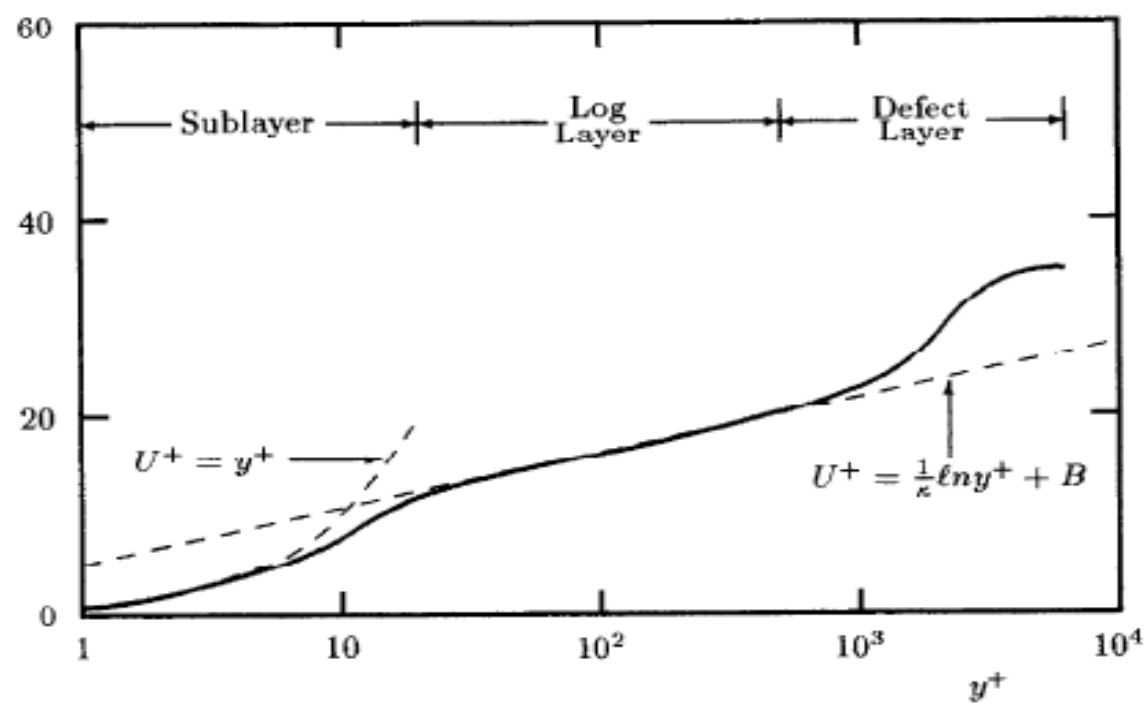

Author: (Wilcox, 1994, pp. 41-53) 


\subsection{Potential Fluid Flow}

For some special cases, the fluid flow could be considered as potential. When the vorticity of the flow and hence the shear stresses are negligible, the Navier-Stokes equation could be simplified, becoming the Poisson equation. In (Newman, 1977, p. 102) one can consider this hypothesis valid far from a thin boundary layer compared with the dimensions involved in the analysis in which the bulk of the fluid is treated as inviscid and irrotational.

The potential fluid flow theory yields a large amount of known analytical solutions, such as the developed for wing and airfoils (Pope A. , 1951B), surface gravity waves, added mass calculation and some other applications. This text includes in Attachment the surface gravity waves theory and the potential fluid flow through a circular cylinder, both derived with the potential fluid flow theory. The first one is necessary to discuss the boundary conditions for a free surface and the second one is a case with a known analytical solution that is used for analysing the effects of the modification of the free surface boundary conditions.

According to the potential fluid flow theory, the velocity vector is derived from a potential function, $\phi$, in which:

$$
u_{i}=\frac{\partial \phi}{\partial x_{i}}
$$

Introducing this definition in the mass conservation equation, is derived the Laplace's equation that governs the potential fluid flow:

$$
\frac{\partial^{2} \phi}{\partial x_{i}^{2}}=0
$$

Substituting the equation ( 4.26 ) in the Navier-Stokes equation, with some algebra, is derived the Bernoulli's equation for the potential fluid flow, which is presented in (Newman, 1977, p. 108) and represents the conservation of energy in the flow.

$$
\frac{\partial \phi}{\partial t}+\frac{1}{2}\left(\frac{\partial \phi}{\partial x_{j}}\right)^{2}+\frac{p}{\rho}+g x_{i}=C t e
$$


The stream function, $\psi$, represents the tangent line to a particle, such as a contour line. The gradient of a stream function is always perpendicular to the velocity vector and this definition is valid for any kind of flow, not only the potential fluid flow. However, for a potential fluid flow, the stream function yields an important mathematical property: Is the conjugate of the potential function, letting to introduce the notion of a complex potential and therefore, the properties of the analytical functions. With the definition of the stream function, the velocity vector is written as:

$$
u_{i}=\frac{\partial \psi}{\partial x_{j}} \epsilon_{i j k}
$$

The complex potential function, $F(z)$, is introduced, in which the potential function, $\phi$, is the real part of the complex potential function and the streamline is the imaginary part of the complex potential function, as follows:

$$
F(z)=\phi(x, y)+i \psi(x, y)
$$

Where:

$$
\begin{array}{ll}
z & \text { Is a complex number } z=x+i y ; \\
i & \text { Is the imaginary number }\left(i^{2}=-1\right) .
\end{array}
$$

The introduction of the complex potential function allows the usage of conformal mapping, useful for deriving the potential fluid flow through solid bodies. 


\subsection{Solid Mechanics for Orthotropic Bodies}

An alternative for a free surface model, proposed by (Mattavo \& Assi, 2017) employs an orthotropic solid to evaluate the free surface effects in the flow around a circular cylinder. They proposed that the same restoring effect could be achieved using a mechanical spring effect instead of the gravity force. Hence, the basics of solid mechanics for orthotropic bodies is reviewed.

The following Equation is valid for any continuous media. It states the equilibrium between the inertia and forces and is known as Newton's second law for particles. The left hand of the Equation represents the inertial term and the right hand represents the sum between body forces and surface forces. In this analysis, only conservative body forces are considered, hence, they are derived from a potential function.

$$
\rho \frac{\partial^{2} u_{i}}{\partial t^{2}}=\frac{\partial \mathcal{V}}{\partial x_{i}}+\frac{\partial \tau_{i j}}{\partial x_{j}}
$$

Where:

$u_{i} \quad$ Is the displacement of the particles in a Lagrangian reference frame;

$v \quad$ Is the body force potential;

$\tau_{i j} \quad$ Is the stress tensor.

Defining the strain tensor as the gradient of the displacement vector and decomposing it in two parts, one symmetric, called strain tensor, and the other antisymmetric, called infinitesimal rotation tensor.

$$
\frac{\partial u_{i}}{\partial x_{j}}=\tilde{E}_{i j}+\widetilde{W}_{i j}=\frac{1}{2}\left(\frac{\partial u_{i}}{\partial x_{j}}+\frac{\partial u_{j}}{\partial x_{i}}\right)+\frac{1}{2}\left(\frac{\partial u_{i}}{\partial x_{j}}-\frac{u_{j}}{\partial x_{i}}\right)
$$

Where:

$\tilde{E}_{i j}, \quad$ Is the strain tensor;

$\widetilde{W}_{i j} \quad$ Is the infinitesimal rotation tensor. 
In linear elastic bodies, only the $\tilde{E}_{i j}$ is taken in account. For a general solid, there is a constitutive equation that relates stress and strain and it is found in (Lai, Krempl, \& Ruben, 2010, p. 204) and is written below:

$$
\tau_{i j}=C_{i j k l} . \tilde{E}_{i j}
$$

Where:

$C_{i j k l} \quad$ Is the stiffness tensor for a linear elastic solid.

For a linear elastic orthotropic solid, the constitutive equation is simplified. An orthotropic material holds three orthogonal symmetry planes and this property is applied in the stiffness tensor. The strain tensor could be divided in two parts: elongation strain and shearing strain, as follows in the equations below:

$$
\begin{gathered}
\varepsilon_{i}=\frac{\partial u_{i}}{\partial x_{i}} \\
\gamma_{i j}=\frac{\partial u_{i}}{\partial x_{j}}+\frac{\partial u_{j}}{\partial x_{i}}
\end{gathered}
$$

Where:$$
\varepsilon_{i} \quad \text { Is the elongation strain; }
$$$$
\gamma_{i j} \quad \text { Is the shearing strain. }
$$

With the definition of elongation and shearing strain, it is possible to relate stress and strain for an orthotropic solid material according to the equations below:

$$
\begin{gathered}
\varepsilon_{i}=\delta_{i j} \frac{\sigma_{i}}{E_{i}}+\left(\delta_{i j}-1\right) \frac{u_{i j} \sigma_{j}}{E_{j}} \\
\gamma_{i j}=\frac{\tau_{i j}}{G_{i j}}
\end{gathered}
$$

Where:

$E_{i} \quad$ Is the orthotropic elasticity modulus;

$G_{i j} \quad$ Is the shear modulus. 
For a two-dimensional problem, It is defined too the Airy's stress function, $\Phi_{A}$, according to (Timoshenko \& Goodier, 2010, pp. 31-33). All stress components are derived from this single function. Therefore, it yields a mathematical advantage in the solid mechanics problems solving, since just a single function must be found. For transient analysis, a modification in the definition of stresses is performed, including a term related to the second derivative with respect to the time in its definition, shown in (Timoshenko \& Goodier, 2010, pp. 485-513).

$$
\begin{gathered}
\sigma_{x}=\frac{\partial^{2} \Phi_{A}}{\partial z^{2}}+\mathcal{V}+\rho \frac{\partial^{2} \Phi_{D}}{\partial t^{2}} \\
\sigma_{z}=\frac{\partial^{2} \Phi_{A}}{\partial x^{2}}+\mathcal{V}+\rho \frac{\partial^{2} \Phi_{D}}{\partial t^{2}} \\
\tau_{x z}=-\frac{\partial^{2} \Phi_{A}}{\partial x \partial z}
\end{gathered}
$$

Where:

$\Phi_{D} \quad$ Is the inertial potential.

For a two-dimensional approach, only the displacements in the $\boldsymbol{x z}$ plane are considered, leading to a plane state of displacements. For this case, the boundary conditions are: $\varepsilon_{y}=\gamma_{x y}=\gamma_{y z}=0$. Hence, is possible to write the stress in y direction in function of stresses in $x$ and $z$ directions. By the substitution of these results in the Equation ( 4.36 ) is shown that:

$$
\begin{aligned}
& \varepsilon_{x}=\left(1-v_{x y}^{2}\right) \frac{\sigma_{x}}{E_{x}}+\left(v_{y z} v_{x y}-v_{x z}\right) \frac{\sigma_{z}}{E_{z}} \\
& \varepsilon_{z}=\left(1-v_{y z}^{2}\right) \frac{\sigma_{z}}{E_{z}}+\left(v_{y z} v_{x y}-v_{x z}\right) \frac{\sigma_{x}}{E_{x}}
\end{aligned}
$$

It is possible to relate the linear strain components with the shear strain components from the definition of $\varepsilon_{i}$ and $\gamma_{i j}$ according to the equations ( 4.34$)$ and ( 4.35 ). By deriving twice $\varepsilon_{i}$ with respect to $x_{j}$ and deriving twice $\varepsilon_{j}$ with respect to $x_{i}$ and summing these two components, it is deduced the following expression:

$$
\frac{\partial^{2} \varepsilon_{i}}{\partial x_{j}^{2}}+\frac{\partial^{2} \varepsilon_{j}}{\partial x_{i}^{2}}=\frac{\partial^{3} u_{i}}{\partial x_{i} \partial x_{j}^{2}}+\frac{\partial^{3} u_{j}}{\partial x_{i}^{2} \partial x_{j}}
$$


The same expression is obtained by deriving the expression ( 4.35 ).one time with respect to $x_{i}$ and once with respect to $x_{j}$. Hence, is derived an expression relating the strain components $\varepsilon_{i}$ and $\varepsilon_{j}$ with $\gamma_{i j}$. Since the analysis focuses on a two-dimensional state of deformations, the following expression relates $\varepsilon_{x}$ and $\varepsilon_{z}$ with $\gamma_{x z}$.

$$
\frac{\partial^{2} \varepsilon_{x}}{\partial z^{2}}+\frac{\partial^{2} \varepsilon_{z}}{\partial x^{2}}=\frac{\partial^{2} \gamma_{x z}}{\partial x \partial z}
$$

Substituting the expression. (4.37) for $\gamma_{x z},(4.41)$ for $\varepsilon_{x}$ and (4.42) for $\varepsilon_{z}$ in the equation (4.44), it is obtained the following equation, relating $\sigma_{x}$ and $\sigma_{z}$ with $\tau_{x z}$.:

$$
\begin{aligned}
\frac{\partial^{2}}{\partial z^{2}}\left[\left(1-v_{x y}^{2}\right)\right. & \left.\frac{\sigma_{x}}{E_{x}}+\left(v_{y z} v_{x y}-v_{x z}\right) \frac{\sigma_{z}}{E_{z}}\right] \\
& +\frac{\partial^{2}}{\partial x^{2}}\left[\left(1-v_{y z}^{2}\right) \frac{\sigma_{z}}{E_{z}}+\left(v_{y z} v_{x y}-v_{x z}\right) \frac{\sigma_{x}}{E_{x}}\right]=\frac{\partial^{2}}{\partial x \partial z}\left(\frac{\tau_{x z}}{G_{x z}}\right)
\end{aligned}
$$

Substituting the stresses by its definition based on modified Airy's stress function, according to ( 4.38 ), ( 4.39 ) and ( 4.40 ), and rearranging the terms is deduced the differential equation for Airy's stress function in orthotropic solids.

$$
\begin{aligned}
\frac{\left(1-v_{x y}^{2}\right)}{E_{x}} \frac{\partial^{4} \Phi_{A}}{\partial z^{4}} & +\left[\frac{1}{G_{x z}}-\left(v_{y z} v_{x y}-v_{x z}\right)\left(\frac{1}{E_{x}}+\frac{1}{E_{z}}\right)\right] \frac{\partial^{4} \Phi_{A}}{\partial x^{2} \partial z^{2}}+\frac{\left(1-v_{y z}^{2}\right)}{E_{z}} \frac{\partial^{4} \Phi_{A}}{\partial x^{4}} \\
& =-\left\{\left[\frac{\left(1-v_{x y}^{2}\right)}{E_{x}}-\frac{\left(v_{y z} v_{x y}-v_{x z}\right)}{E_{z}}\right] \frac{\partial^{2}}{\partial z^{2}}\right. \\
& \left.+\left[\frac{\left(1-v_{y z}^{2}\right)}{E_{z}}-\frac{\left(v_{y z} v_{x y}-v_{x z}\right)}{E_{x}}\right] \frac{\partial^{2}}{\partial x^{2}}\right\}\left(\mathcal{V}+\rho \frac{\partial^{2} \Phi_{D}}{\partial t^{2}}\right)
\end{aligned}
$$

For simplicity, is defined that $E_{x}=E_{z}=E, \quad v_{x y}=v_{y z}=v_{x z}=v$ and $G_{x z}=G$. Substituting in the equation ( 4.46 ):

$$
\begin{array}{r}
\frac{G}{E}\left(1-v^{2}\right) \frac{\partial^{4} \Phi_{A}}{\partial z^{4}}+\left[1+\frac{G}{E} 2 v(1-v)\right] \frac{\partial^{4} \Phi_{A}}{\partial x^{2} \partial z^{2}}+\frac{G}{E}\left(1-v^{2}\right) \frac{\partial^{4} \Phi_{A}}{\partial x^{4}} \\
=-\frac{G}{E}\left(1+v-2 v^{2}\right)\left(\frac{\partial^{2}}{\partial z^{2}}+\frac{\partial^{2}}{\partial x^{2}}\right)\left(v+\rho \frac{\partial^{2} \Phi_{D}}{\partial t^{2}}\right)
\end{array}
$$

For a solid with the shear modulus small if compared with elasticity modulus, the equation ( 4.47 ) becomes:

$$
\frac{\partial^{4} \Phi_{A}}{\partial x^{2} \partial z^{2}}=0
$$


Since the orthotropic solid is implemented to represents the behaviour of an elastic free surface, in which there is no transmission of shear, the hypothesis of shear modulus being negligible if compared with elasticity modulus is valid. Hence, for the analytical model of the free surface and for the elasticity modulus calculation the equation ( 4.48 ) will be considered.

\subsection{Computational Methods}

This section presents the computational methodologies used in this work. For the Computational Fluid Mechanics (CFD), it is used the Finite Volume Method (FVM) implemented in the commercial code Ansys CFX $17.0 \AA$ and for the structural analysis it is used the Finite Element Method implemented in the commercial code ANSYS Mechanical ${ }^{\circledR}$.

A brief FVM review is performed in the section 4.4.1 highlighting the mathematical basis of the methodology and the choice of the input parameters necessary to a CFD simulation such as interpolation scheme, convergence criteria and mesh quality. Further, it is performed a brief FEM review including the mathematical basis of the methodology and the analysis of the hexahedral element, which is used in the present word.

\subsubsection{Computational Fluid Dynamics (CFD)}

Several methodologies are employed for the flow through tidal current turbines flow analysis. In the section 3.1.2, (Pinón et al., 2012) performed an analysis with a particle method which overpredicted the power coefficient for higher TSR's. In the literature, it is found meshless also for the analysis. (Salvatore et. al, 2018) proposed a Boundary Element method based on an Integral methodology for an inviscid flow and estimated the shear stresses in the turbine walls with the formulation used for a flat plat in both laminar and turbulent flows. The method predicted the peak power coefficient with an error lower than $2 \%$, but overpredicted the power coefficient for higher TSR's. Another disadvantage of such methodology is that no free surface effects were considered and since the fluid domain is not modeled, the wake analysis was not performed. 
Even with these methodologies, most of the author analysed the flow through tidal current turbines using a Finite Volume Method, as discussed previously. With such methodology is possible to analyse the whole fluid domain including turbulence, free surface and confinement effects. A multiphasic flow such as the Volume of Fluid (VOF) for free surface analysis. Hence, this methodology is applied in the present work and is discussed in this chapter.

The equations presented in the section 4.1 could be represented in the conservative form, as discussed in (Pantankar, 1980, pp. 15-17). In this general form, the transport equation of a scalar quantity $\varphi$ is given by:

$$
\frac{\partial}{\partial t}(\rho \varphi)+\frac{\partial}{\partial x_{j}}\left(\rho u_{j} \varphi\right)=\frac{\partial}{\partial x_{j}}\left[\Gamma^{\varphi} \frac{\partial}{\partial x_{j}}(\varphi)\right]+S^{\varphi}
$$

Where:

$$
\begin{array}{cl}
\varphi & \text { Is a scalar quantity; } \\
\Gamma^{\varphi} & \text { Is the diffusion coefficient related to this scalar quantity; } \\
\frac{\partial}{\partial t}(\rho \varphi) & \text { Is the local rate of change; } \\
\frac{\partial}{\partial x_{j}}\left(\rho u_{j} \varphi\right) & \text { Is the advective rate of change; } \\
\frac{\partial}{\partial x_{j}}\left[\Gamma^{\varphi} \frac{\partial}{\partial x_{j}}(\varphi)\right] & \text { Is the diffusive term; } \\
S^{\varphi} & \text { Is the source term. }
\end{array}
$$

In an incompressible, viscous and turbulent flow, such the analysed in this text, using the k-w-SST for turbulence modelling, there are six differential equations to be solved and all of then written in the same form as the equation (4.49). In order to solve this mathematical problem, these equations must be discretized in order to be solved in a finite quantity of points in the fluid domain. In (Maliska, 2014, pp. 195-196) this equation is integrated for a general fluid element. For a node " $i$ ", the equation becomes: 
Figure 18 - Control Volume Integration for Element Based Finite Volume Method

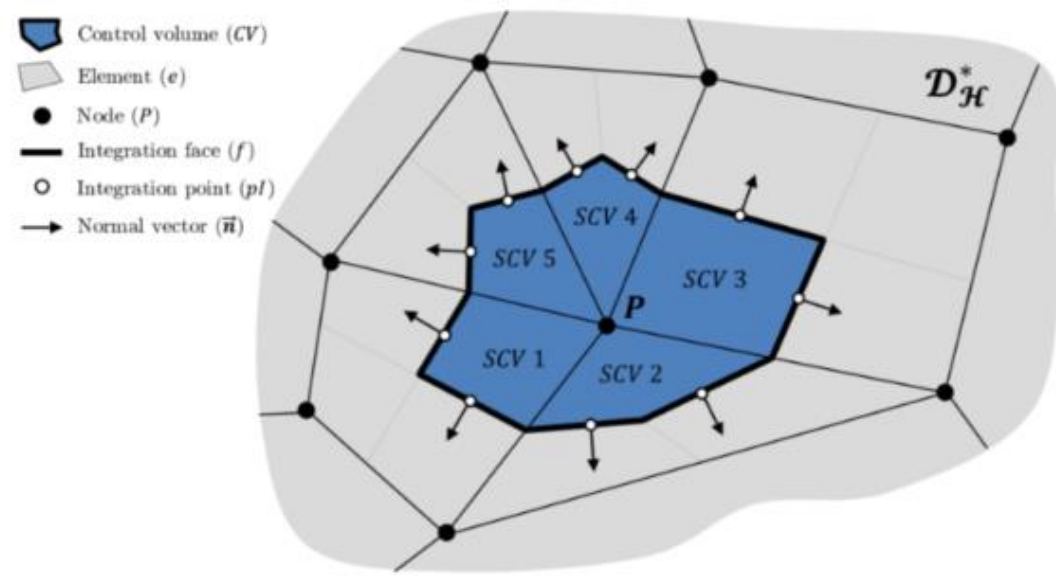

Source: (Profito, 2015)

$$
\begin{aligned}
\frac{M_{P} \varphi_{i}-M_{P}^{0} \varphi_{i}^{0}}{\Delta t} & =-\sum_{s_{j}}[\rho(\vec{V} \cdot \vec{n}) \varphi \Delta S]_{S_{j}}+\sum_{s_{j}}\left[\Gamma^{\varphi}(\nabla \varphi \cdot \vec{n}) \Delta S\right]_{s_{j}} \\
& +\sum_{s_{j}}\left(S_{i}^{\varphi} \varphi_{i}+S_{C}^{\varphi}\right) \Delta \mathrm{V}
\end{aligned}
$$

Where:

$M_{P} \quad$ Is the mass of the element in the analysed time step;

$\varphi_{i} \quad$ Is the nodal value of $\varphi$ in the node "i" in the analysed time step;

$M_{P}^{0} \quad$ Is the mass of the element in the previous time step;

$\varphi_{i}^{0} \quad$ Is the nodal value of $\varphi$ in the node "i" in the previous time step;

$\Delta t \quad$ Is the time step of the integration;

$\vec{V} \quad$ Is the Velocity Vector;

$\vec{n} \quad$ Is the normal vector to a control surface;

$s_{j} \quad$ Is the control surface "j";

$\Delta S \quad$ Is the control surface total area;

$S_{i}^{\varphi} \quad$ Is the source term in the node "i" in the analysed time step;

$S_{C}^{\varphi} \quad$ Is the independent part of the linearized source term;

$\Delta \mathrm{V} \quad$ Is the total volume of the element "i". 
The Figure 18 illustrates the integrated domain for generating the equation (4.50). The point " $P$ " shown in the figure represents the node " $\mathrm{i}$ " used in the equation above. Besides, for calculating the values of $\varphi$ in the subdomain boundaries, represented by $s_{j}$ in the equation and by a thick line in the figure, is cecessary to interpolate the value of $\varphi$ using the values of this variable in the neighbour nodes. In the diffusive term, the gradient of $\varphi$ must be calculated also. Hence, the derivatives of $\varphi$ must be calculated and since they are not known a priori, a proper scheme of interpolation shall be careful chosen.

In (Maliska, 2014, pp. 75 -104) several interpolation functions are discussed for the diffusive and advective components. The dependency of the dominant phenomena in the flow is a relevant factor in the choice of the interpolation scheme. The upwind scheme, for example, which calculates the derivative using only the upstream node following the flow direction smooths gradients and could lead to a problem called numerical diffusion.

Some interpolation functions consider the effects of the advective term. For example, in a flow in which the advective transport is dominant if compared with diffusion, which occurs in regions far from boundary layer in high Reynolds flows, the downstream values of a certain variable $\varphi$ are more representative than the upstream and points which are not in the streamwise direction. Hence, a weighted scheme such as Weighted Upstream Differencing Scheme - Extended (WUDS-E) is a valuable option.

For incompressible flows, the pressure and velocity are decoupled a priori. In the momentum conservation equation, the pressure gradient is used as a source for calculating the velocity components. However, for calculating the pressure field, there is not a single equation in which the pressure term could be separated. Deriving the equation ( 4.7 ) with respect to $x_{i}$ is found the Poisson Equation for pressure, as follows:

$$
\frac{\partial^{2} \overline{\mathrm{p}}}{\partial x_{i}^{2}}=-\rho\left[\frac{\partial \bar{u}_{i}}{\partial x_{j}} \frac{\partial \bar{u}_{j}}{\partial x_{i}}+\frac{\partial^{2}}{\partial x_{j} \partial x_{i}}\left(\overline{\bar{u}_{i}^{\prime} \mathbf{u}_{j}^{\prime}}\right)\right]
$$


Some pressure-velocity coupling methods such as SIMPLE (Semi-Implicit Linked Equations) and SIMPLER (Semi-Implicit Linked Equations-Revised) makes use of Poisson equation for pressure to correct the pressure field. Besides, the most known methods for solving a CFD problem through FVM makes use of an iterative solver. Since the Navier-Stokes equations are not linear. Hence, the pressure-velocity coupling methods are iterative and needs a convergence criterium.

The standard method in the commercial code ANSYS CFX 17.0® is the Fourth Order Rhie-Chow Method (Rhie \& Chow, 1982). This coupling is applied for a co-located grid in which the control volume is the same for all transport equations. The method uses the continuity equation to solve the pressure field.

\subsubsection{Finite Element Method}

The finite element method is often used for structures and heat transfer problems. There are high order methods applied to fluids, however, this approach is not shown in this work since the scope is limited to the Finite Element application on structures.

From (Hughes, 2000), it is known that a boundary value problem could be analysed by a strong, differential formulation and through a weak, integral formulation. And both methods are mathematically equivalent. The Finite Element Method uses the integral approach for discretize the system through interpolation functions, so-called shape functions, and provide trough integrals along the element domain, formulas for the stiffness and load parameters that describes the system.

From (Timoshenko \& Goodier, 2010, pp. 235-277), for a three dimensional elastic solid, the differential approach for analysing the deformations came from newton second law for infinitesimal values. This equation relates by equilibrium of forces, the relation between stresses and body forces. Analysing the stress field in the solid boundaries with the compatibility of strain and by the equilibrium of forces, the deformation field along the body is found. However, this section is interested on analyse the integral formulations of solid mechanics. From (Timoshenko \& Goodier, 2010, pp. 235-277), the strain potential energy stored in an elastic body, contained in the domain $\Omega_{D}$, it is given by the following integral: 


$$
U_{\text {strain }}=\frac{1}{2} \int_{\Omega_{D}}\{\sigma\}^{T} \cdot\{\varepsilon\} \cdot d \Omega_{D}
$$

Where:

$\mathcal{U}_{\text {strain }}$ Is elastic potential energy due to a solid deformation;

$\{\sigma\} \quad$ Is the stress vector;

$\{\varepsilon\} \quad$ Is the strain vector;

$\Omega_{D} \quad$ Is the solid domain.

For a linear elastic body, the stress and strain are related by a linear transformation. Let $[\mathcal{D}]$ be the matrix which contains the relation between stress and strain. The integral becomes:

$$
U_{\text {strain }}=\frac{1}{2} \int_{\Omega_{D}}\{\varepsilon\}^{T}[\mathcal{D}] \cdot\{\varepsilon\} \cdot d \Omega_{D}
$$

The external forces produce an amount of work that by energy conservation must be equal to the stored strain energy. Dividing the work due to external forces by work due to body forces, $\mathcal{W}_{B}$, and work due to surface forces, $\mathcal{W}_{S}$, and calling the domain boundary as $\partial \Omega_{D}$, the work due to external forces is given by:

$$
\mathcal{W}=\mathcal{W}_{B}+\mathcal{W}_{S}=\int_{\Omega_{D}} X_{i} u_{i} \cdot d \Omega_{D}+\int_{\partial \Omega_{D}} S_{i} u_{i} \cdot d \partial \Omega_{D}
$$

Since the total work is equal to the total strain energy, the equation ( 4.53 ) is equal to the equation ( 4.54 ).

$$
\frac{1}{2} \int_{\Omega_{D}}\{\varepsilon\}^{T}[\mathcal{D}] \cdot\{\varepsilon\} \cdot d \Omega_{D}=\int_{\Omega_{D}} X_{i} u_{i} \cdot d \Omega_{D}+\int_{\partial \Omega_{D}} S_{i} u_{i} \cdot d \partial \Omega_{D}
$$

As described in the section 4.3, the strain is related to the displacement by differential operators. However, the displacement field, $u_{i}$, it is not known a priori. Hence, the domain is divided into small parts indeed, and for each part, it is given a set of functions with value equal to zero outside the element domain, which 
interpolates the displacement values from given nodes to calculate the displacement inside the element domain. Calling $\mathcal{N}_{i}{ }^{e}$ the set of shape functions associated with each element, the differential operator which relates stress and strain could be turned into an algebraic operator, $[\mathcal{B}]$. Hence, the equation ( 4.55 ) becomes:

$$
\frac{1}{2} \int_{\Omega_{D}}\{u\}^{T}[\mathcal{B}]^{t}[\mathcal{D}] .[\mathcal{B}]\{u\} . d \Omega_{D}=\int_{\Omega_{D}} X_{i}[\mathcal{N}]\{u\} . d \Omega_{D}+\int_{\partial \Omega_{D}} S_{i}[\mathcal{N}]\{u\} . d \partial \Omega_{D}
$$

Since the nodal displacement vector is constant, it could be taken out from the integrals:

$$
\frac{1}{2}\{u\}^{T} \int_{\Omega_{D}}[\mathcal{B}]^{t}[\mathcal{D}] \cdot[\mathcal{B}] \cdot d \Omega_{D}\{u\}=\{u\}^{T}\left\{\int_{\Omega_{D}} X_{i}[\mathcal{N}] \cdot d \Omega_{D}+\int_{\partial \Omega_{D}} S_{i}[\mathcal{N}] \cdot d \partial \Omega_{D}\right\}
$$

According to the minimum complementary potential energy theorem, the displacements are such that the difference between strain potential energy and stored external work is stationary. Hence, the variational of the previous equation is equal to zero. It yields:

$$
\{\delta u\}^{T}\left\{\left[\int_{\Omega_{D}}[\mathcal{B}]^{t}[\mathcal{D}] \cdot[\mathcal{B}] \cdot d \Omega_{D}\right]\{u\}-\int_{\Omega_{D}} X_{i}[\mathcal{N}] \cdot d \Omega_{D}-\int_{\partial \Omega_{D}} S_{i}[\mathcal{N}] \cdot d \partial \Omega_{D}\right\}=0
$$

Therefore, the displacement vector must fulfil the following relation:

$$
\left[\int_{\Omega_{D}}[\mathcal{B}]^{t}[\mathcal{D}] .[\mathcal{B}] \cdot d \Omega_{D}\right]\{u\}=\int_{\Omega_{D}} X_{i}[\mathcal{N}] \cdot d \Omega_{D}+\int_{\partial \Omega_{D}} S_{i}[\mathcal{N}] . d \partial \Omega_{D}
$$

The commercial tool, Ansys Workbench® uses exactly this formulation for compose their structural matrices. The matrices from ( 4.59 ) could be assembled by dividing the integration through the domain, calculating local stiffness matrix and force vector for each element and assembling then for solving the problem. The problem becomes:

$$
[K]\{u\}=\{F\}
$$


Where:

$[K] \quad$ Is the global stiffness matrix;

$\{F\} \quad$ Is the global nodal force vector.

Calling $\Omega_{e}$, the element domain, and $\partial \Omega_{D}$ the element boundary, the element stiffness and its local force vector is given by:

$$
\begin{gathered}
{\left[K_{e}\right]=\int_{\Omega_{e}}[\mathcal{B}]^{t}[\mathcal{D}] \cdot[\mathcal{B}] \cdot d \Omega_{e}} \\
\left\{F_{e}\right\}=\int_{\Omega_{e}} X_{i}[\mathcal{N}] \cdot d \Omega_{e}+\int_{\partial \Omega_{e}} S_{i}[\mathcal{N}] \cdot d \partial \Omega_{e}
\end{gathered}
$$

In this work, due to the regularity of the solid domain, it is possible to use a mesh constituted elements with an intermediate node on each edge and therefore, have an additional degree of interpolation if compared with standard hexahedral without intermediate nodes.

The interpolation has a degree equal to 2 for each coordinate system, $r, s, t$, assuming for the shape function associated with the node $i$, the following general formulation:

$$
\mathcal{N}_{i}(r, s, t)=A_{i}\left(a_{r}^{i} r^{2}+b_{r}^{i} r+c_{r}^{i}\right)\left(a_{s}^{i} s^{2}+b_{s}^{i} s+c_{s}^{i}\right)\left(a_{t}^{i} t^{2}+b_{t}^{i} t+c_{t}^{i}\right)
$$

The shape function for this finite element have the same property of the shape function used in the finite volume method interpolation and its formulation is extent and presented in Ansys User's Guide (ANSYS Inc., 2016).

Figure 19 - Solid 186 Element
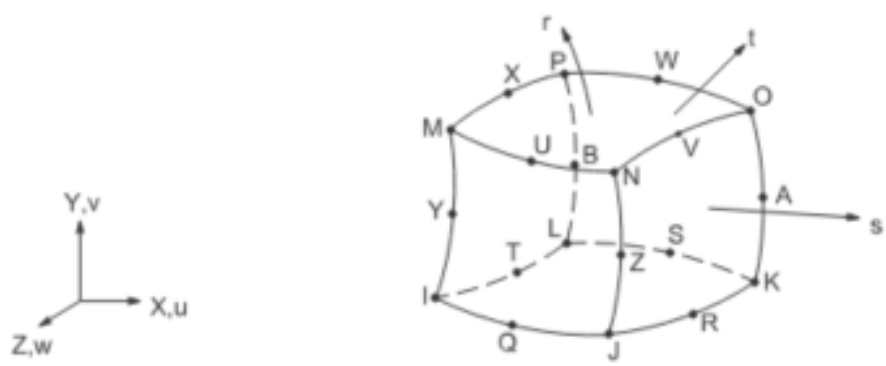

Source: (ANSYS Inc., 2016) 


\section{Chapter 5. Methodology}

Since this text goal is to evaluate the flow through an axial tidal current turbine with an elastic wall approach for the free surface modelling, the methodology focuses on validating a CFD simulation for a tidal current turbine against experimental results for an open flow boundary condition and then analyze the differences in the flow with the introduction of different boundary conditions and confinements such as the free surface effect. Moreover, several analytical solutions containing the modification of the free surface boundary condition are performed in order to check if the adopted hypothesis leads to consistent results if applied to simple flows.

A summary of the methodology is presented in Figure 20. It is shown that the work is conducted by two different paths. The first one consists in validating a CFD simulation against experimental data from (Bahaj, W.M.J., \& McCann, 2007). The second one consists to develop the alternative free surface and analyze its effects by both analytical and computational methodologies.

Before start the validation process, since the simulation consists in a blade resolved CFD, the turbine geometry is modeled in a CAD program following the data from (Bahaj, W.M.J., \& McCann, 2007). Then, a parametric domain with adjustable lengths, diameters, height, and width is created.

Figure 20 - Methodology summary

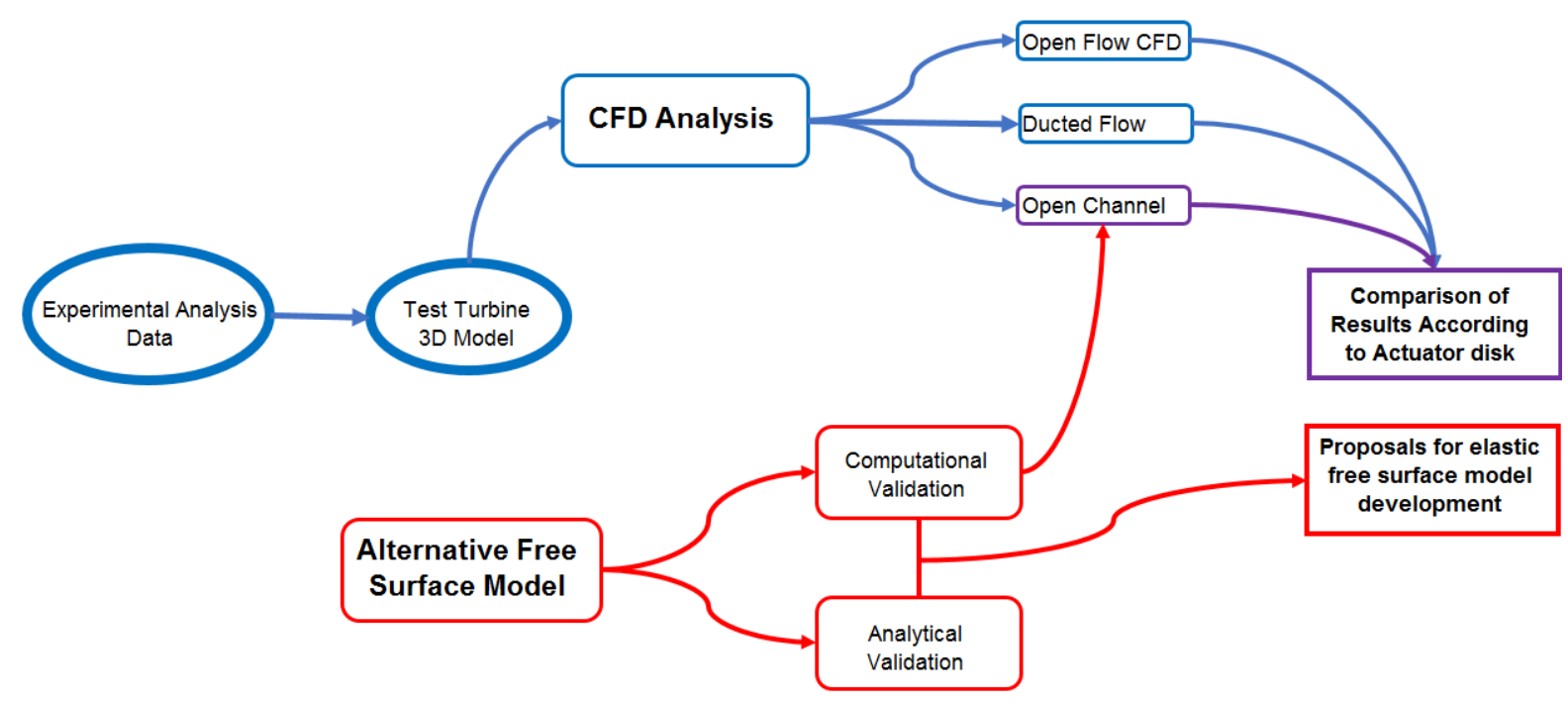


The CFD validation consists in comparing the results from the CFD model with the experimental results. During this phase, the following tests are performed:

- Mesh Sensitivity Analysis;

- Turbulence Model Analysis;

- Upstream and Downstream Lengths and Domain Diameter Analysis;

The results are compared between themselves to analyze the mentioned parameters influence in the results, and then, the CFD results are compared with the experimental data related to power and thrust coefficients. It is known that there are several parameters to be analyzed regarding this simulation, however, since there is no available data in (Bahaj, W.M.J., \& McCann, 2007) and this text is focused on the tidal current turbine performance, an analysis including the effects of domain dimensions in parameters such as wake recovery is not presented.

After validating the CFD analysis for an open domain, the analysis is performed for several TSR values to ensure that the methodology is valid for the experimental range of results, predicting the peak power coefficient and decreasing the power coefficient for high rotation values.

After defining the discussed simulation parameters, it is modeled the simulation for those domains:

- Open Domain;

- Confined Domain;

- Open Channel Domain.

For these different boundary conditions, the power and thrust coefficients are analyzed. It is expected that the confined domain presents the highest power coefficients according to the actuator disk theory.

However, for analyzing the Free Surface, it is required the development of the methodology for including such effects. In this text, the elastic wall method is applied to the problem, and hence a previous study for simpler flows is necessary. 
This elastic free surface is modeled through an orthotropic solid to present a linear spring effect. The hypothesis that were discussed in section 5.3 are highlighted, and the final equation is solved analytically showing that the necessary mechanical properties for the elastic wall modelling are obtained by just following some simple hypothesis.

With the elastic wall modeled, the new boundary conditions are for the following analytical problems that require the free surface boundary condition:

- Linear Wave Propagation;

- Waves due to a Submerged Cylinder;

- Actuator Disk Model for an Open Channel.

The analysis shows that at least for these flows, the boundary condition is sufficient to include the free surface effects. For both linear wave propagation problem and waves due to a Submerged Cylinder, the solution presents the same shape for the free surface as the classic boundary condition. For the actuator disk model for an open channel, which is based on an integral control volume analysis, the forces in the streamwise direction are the same as the classic model and the forces in the vertical direction are different if compared with the classic model just by the buoyancy force, which is efficiently computed by simple analytical methodologies.

The CFD simulation of a submerged cylinder performed is discussed. In this work, the drag in a circular cylinder close to a free surface is computed by both elastic wall methodology and by VOF for free surface analysis.

After those discussions, the developed free surface model is implemented for the axial tidal current turbine, analyzing the method stability, computational time and results in comparison with the confined domain and open domain flows. 


\section{Chapter 6. Free Surface Modelling}

Modelling the Free Surface is known for being a challenge. There is a high number of engineering problems in which there is important the accuracy of such modelling. Included in this countless number of problems, one can highlight: Flow in open channels, flow through ships and oceanic platforms, green water, wave energy harvesting, and others.

The free surface could be treated by both Lagrangian and Eulerian Scheme. The Lagrangian approach is often chosen for non-linear problems such as ship sloshing, associated with green water. However, for many phenomena such as flow in open channels, the Eulerian scheme is a recurrent choice. This approach computes the fluid properties in some domain positions and does not track a single particle as the Lagrangian Scheme. In the context of the Eulerian approach, it is highlighted the Volume of Fluid (VOF) Method. It is a biphasic method in which on each fluid cell, the proportion between the two fluids is calculated, and all properties of the fluid are weighted by the proportion between the mass of each fluid.

One advantage of the VOF is that it is already implemented in commercial tools such as ANSYS CFX® and ANSYS FLUENT®. However, VOF leads to a high computational cost since there is a necessity of including a secondary fluid domain, increasing the number of fluid elements and is required for refining the mesh in the free surface region. Besides, an additional variable: the proportion between the two fluid, is necessary. Hence, an analysis including such methodology is not feasible for some industrial flows without using a cluster.

Hence, it is proposed a different methodology for analyzing the free surface using an elastic wall in the free surface region. The idea behind this methodology is to provide a restoring force with the same effect of the gravitational field, and hence the energy conservation condition is fulfilled. In this chapter, the hypothesis for this modelling are discussed, and the implementation of such boundary condition in simple flows with known analytical solutions is performed to provide the basis for using this method for computing the flow through an axial tidal current turbine close to a free surface. 


\subsection{Elastic Free Surface Model}

The orthotropic body is initially designed for providing the same restoring effect as the gravity in the case of free surface deformations. According to (Newman, Marine Hydrodynamics, 1977-A), the wave potential energy is proportional to the square of the instantaneous free surface deformation. The same result is achieved for a linear spring. Hence, an equivalent spring constant could be deduced.

Moreover, the energy is proportional to the local deformation. Therefore, the solid body must not transmit shear to guarantee the non-loaded portion of solid presents strain potential energy. For present these properties, an orthotropic body is required.

Then, this section is destined to relate the equivalent wave spring with the orthotropic solid mechanical properties and perform some analytical analysis of free surface effects such gravity waves propagation and analyzing the differences between the two free surface models.

\subsubsection{Orthotropic Solid Transversal Stiffness Calculation}

The free surface model requires the following property:

Where:

$$
\delta(x, y)=\frac{1}{k} p(x, y)
$$

$$
\begin{array}{cl}
\delta(x, y) & \text { Solid free end displacement distribution; } \\
p(x, y) & \text { Pressure distribution in the free surface; } \\
\mathrm{k} & \text { Free surface equivalent stiffness. }
\end{array}
$$

For a body with such mechanical behavior is necessary that $G \ll E$, which means that the terms with coefficient $G^{-1}$ are more significant than the terms with coefficient $E^{-1}$, which leads to the equation (5.48). This equation allows the separation of variables, therefore the function $\Phi_{A}$ could be written as the product of two functions, the first one, the function of $x, \Phi_{A x}(x)$, and the other, function of $z, \Phi_{A z}(z)$. Since the body is only loaded in the $z$ direction, it is assumed that there is no stress in $x$ direction. Hence:

$$
\frac{d^{2} \Phi_{A x}}{d x^{2}} \cdot \frac{d^{2} \Phi_{A z}}{d z^{2}}=0 \rightarrow \frac{d^{2} \Phi_{A z}}{d z^{2}}=0
$$


Integrating twice the equation ( 6.2 ) is obtained:

$$
\Phi_{A z}=A_{\Phi} Z+B_{\Phi}
$$

Since the stress in the lower face is equal the negative pressure value and this stress is obtained through the second derivative of $\Phi_{A}$ with respect to $x$, it is given that:

$$
\sigma_{z}\left(x, h_{s}\right)=\left.\frac{\partial^{2} \Phi_{A}}{\partial x^{2}}\right|_{z=h_{S}}=\left(A_{\Phi} h_{s}+B_{\Phi}\right) \cdot \frac{d^{2} \Phi_{A x}}{d x^{2}}=-p(x)
$$

Considering a linear solid model the superposition principle is valid. Therefore, the solution of both stress, strain and displacement fields is obtained by a linear combination between the contribution of each applied load in the solid. Hence, the pressure could be divided in a sin series, hence, solving the equation for the $n-t h$ term of this series, with amplitude $\boldsymbol{P}_{\boldsymbol{n}}$ and wave number $\boldsymbol{k}_{\boldsymbol{n}}$ :

$$
\left(A_{\Phi} h_{s}+B_{\Phi}\right) \cdot \frac{d^{2} \Phi_{A x}}{d x^{2}}=-P_{n} \cdot \sin \left(k_{n} x\right)
$$

Integrating this equation two times with respect to $x$ and neglecting the homogeneous solution terms:

$$
\Phi_{A x}=\frac{P_{n}}{k_{n}^{2}\left(A_{\Phi} h_{s}+B_{\Phi}\right)} \cdot \sin \left(k_{n} x\right) \rightarrow \Phi_{A}(x, z)=\frac{P_{n}}{k_{n}^{2}} \cdot \sin \left(k_{n} x\right)
$$

For this stress function, the stress in $z$ direction is constant and equal to:

$$
\sigma_{z}=\frac{\partial^{2} \Phi_{A}}{\partial x^{2}}=-P_{n} \cdot \sin \left(k_{n} x\right)
$$

Figure 21 - Solid main dimensions and boundary conditions

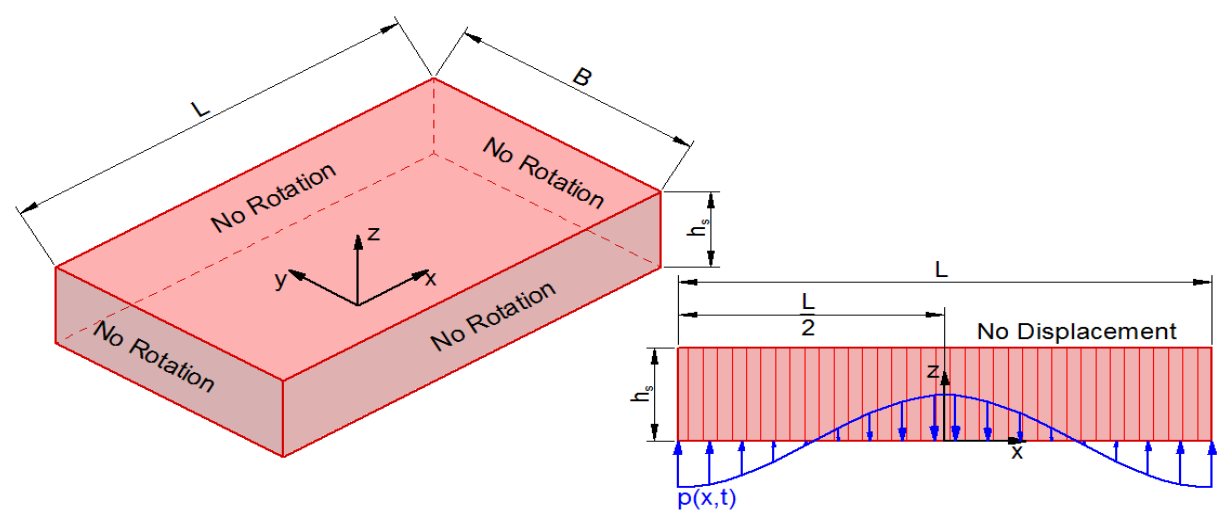

Source: (Mattavo \& Assi, 2017) 
The displacement field is obtained by integrating the strain in z-component:

$$
v_{z}(x, z)=v_{z}(x, 0)+\int_{0}^{z} \frac{\sigma_{z}}{E_{Z}} \cdot d z=-\frac{P_{n} \cdot z}{E_{Z}} \cdot \sin \left(k_{n} x\right)
$$

Considering that the displacement of the free surface, $v_{z}\left(x, h_{s}\right)$, it is proportional to the load, such as a linear spring, it is given that:

$$
v_{z}\left(x, h_{s}\right)=-\frac{P_{n} \cdot h_{s}}{E_{Z}} \cdot \sin \left(k_{n} x\right)=-\frac{P_{n}}{k_{Z}} \cdot \sin \left(k_{n} x\right)
$$

It results in:

$$
\mathrm{k}_{\mathrm{Z}}=\frac{\mathrm{E}_{\mathrm{Z}}}{\mathrm{h}_{\mathrm{s}}}
$$

Moreover, the wave potential energy, $\mathrm{E}_{\mathrm{PG}}^{\mathrm{Gravity} \text { Wave }}$, is given by:

$$
\mathrm{E}_{\mathrm{PG}}^{\text {Gravity Wave }}=\frac{1}{2} \rho \cdot g \cdot \eta^{2}
$$

While the elastic energy due to a spring deformation is given by:

$$
\mathrm{E}_{\mathrm{PG}}^{\text {Spring }}=\frac{1}{2} \mathrm{k}_{\mathrm{z}} \cdot(\Delta \mathrm{z})^{2}
$$

By comparison between these two equations, it is given the following result:

$$
\mathrm{k}_{\mathrm{z}}=\rho \cdot \mathrm{g} \rightarrow \mathrm{E}_{\mathrm{Z}}=\rho \cdot \mathrm{g} \cdot \mathrm{h}_{\mathrm{s}}
$$

Since that for finite element commercial program, ANSYS Mechanical $\AA_{\text {, it is not }}$ allowed the usage of any property equal to zero, it is assumed the shear modulus is equal to $0.1 \%$ of the elasticity modulus and the Poisson coefficient is equal to $10^{-3}$. For simplicity, the properties are considered equal in all directions and the expressions are summarized in the Table 2.

Table 2 - Orthotropic solid mechanical properties (Mattavo \& Assi, 2017)

\begin{tabular}{|c|c|c|}
\hline Property & Symbol & Formula \\
\hline Elasticity Modulus & $E_{x}=E_{y}=E_{z}=E$ & $E=\rho . g \cdot h_{s}$ \\
\hline Shear Modulus & $G_{x y}=G_{x z}=G_{y z}=G$ & $G=E \times 10^{-3}$ \\
\hline Poisson Coefficient & $v_{x y}=v_{x z}=v_{y z}=v$ & $v=10^{-3}$ \\
\hline Solid Pre-Stress & $p_{a}$ & $p_{a}=\rho . g \cdot H_{f}$ \\
\hline
\end{tabular}




\subsection{Consequences in fluid flow due to substitution of buoyancy for elastic free surface}

\subsubsection{Waves due to a Submerged Cylinder}

Considering a submerged cylinder in an infinite depth channel with the free surface with the velocity potential according to the equation (A.1.4). By hypothesis, the depth of the cylinder, $f$, it is sufficiently large if compared with cylinder radius, $R$. In the plane $x z$, the real part of velocity potential becomes:

$$
\Phi(x, z)=-U x\left[1+\frac{R^{2}}{x^{2}+(z+f)^{2}}\right]
$$

This potential has an equivalent integral formulation, according to the next equation:

$$
\Phi(x, z)=-U x-U R^{2} \int_{0}^{\infty} e^{-k(y+f)} \cdot \sin (k x) \cdot d k
$$

Considering the high depth of the cylinder, it is assumed that the induced velocities due to the free surface are negligible on the cylinder. Therefore, the free surface velocity potential must decrease with the depth. According to (Lamb, 1879B, pp. 410$413)$, the velocity potential due to free surface deformations is given by:

$$
x(x, z)=\int_{0}^{\infty} \alpha(k) \cdot e^{k y} \cdot \sin (k x) \cdot d k
$$

Where:

$x \quad$ Is the disturbance in free surface potential;

$\alpha \quad$ Is the disturbance amplitude associated with a wave number $k$

The free surface is given by a cosine series:

$$
\eta(x)=\lim _{\Delta k \rightarrow 0} \sum_{n=0}^{+\infty} \beta_{n} \cdot \cos (n x \Delta k)=\int_{0}^{\infty} \beta(k) \cdot \cos (k x) \cdot d k
$$

Where:

$\eta \quad$ Is the free surface shape function;

$\beta \quad$ Is the free surface amplitude associated with a wave number $k$ 
Figure 22 - Schematic drawing for potential flow around a circular cylinder in an infinite depth open channel

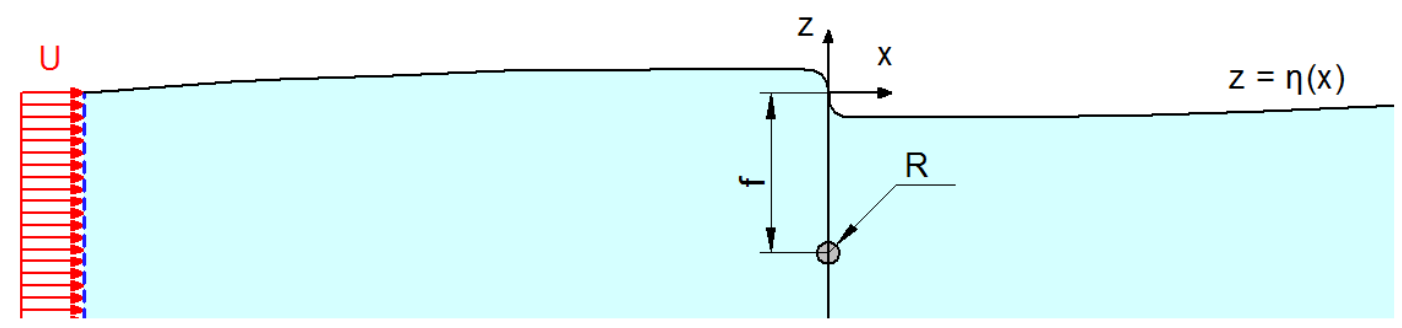

Since both potentials due to cylinder and free surface vanishes at infinite depths, there are two boundary conditions left, the kinematic boundary condition, in which the fluid flow is tangent to the free surface deformation and the other, so-called dynamic boundary condition states the pressure is constant along the free surface, according to the Bernoulli's equation. The kinematic boundary condition assumes that on free surface, the horizontal velocity component is equal to the free stream velocity and the vertical component is equal to:

$$
\frac{\partial}{\partial y}(\Phi+\chi)=-U \frac{\partial \eta}{\partial x}
$$

From (Lamb, 1879B), the vertical component of velocity is negligible since the free surface deformation and then its derivative with respect to $x$ are small. Hence, the dynamic condition, in which the pressure is constant along the free surface is given by:

$$
\frac{p}{\rho}=-g z-\frac{1}{2}\left[\frac{\partial}{\partial x}(\Phi+\chi)\right]^{2}
$$

However, neglecting the buoyancy effects and assuming a pressure on free surface proportional to the free surface deformation, using a stiffness according to the equation ( 6.13 ), it is given that:

$$
p(x, \eta)=\rho \cdot g \cdot \eta
$$

From the Bernoulli's equation on the free surface, in a steady state fluid flow:

$$
\frac{1}{2}\left(\frac{\partial \phi}{\partial x_{j}}\right)^{2}+\frac{p}{\rho}=C t e
$$


By the same assumption of small vertical velocity component, substituting the equation (6.20) and inverting the signal of the equation:

$$
-C t e=-g \eta-\frac{1}{2}\left[\frac{\partial}{\partial x}(\Phi+x)\right]^{2}
$$

By comparison, both pressure term on equation ( 6.19) and the constant term in equation ( 6.22 ) are constants along to the $x$ axis. That means that both derivatives with respect to $x$ are equal to zero. Which means that:

$$
\frac{\partial}{\partial x}\left\{g \eta+\frac{1}{2}\left[\frac{\partial}{\partial x}(\Phi+x)\right]^{2}\right\}=0
$$

Developing the previous equation:

$$
\begin{aligned}
\frac{\partial}{\partial x}\left\{g \int_{0}^{\infty} \beta(k) \cdot \cos (k x) \cdot d k\right. \\
\left.\quad+\frac{U^{2}}{2}\left[\left(1+R^{2} \int_{0}^{\infty} k \cdot \sin (k x)\left[e^{-k(y+f)}+\alpha(k) \cdot e^{k y}\right] \cdot d k\right)\right]^{2}\right\}=0
\end{aligned}
$$

Neglecting the square of the integral $\int_{0}^{\infty} k \cdot \sin (k x)\left[e^{-k(y+f)}+\alpha(k) \cdot e^{k y}\right] \cdot d k$, assuming that the disturbance effects are negligible if compared with free stream:

$$
\begin{aligned}
\frac{\partial}{\partial x}\left\{g \int_{0}^{\infty} \beta(k) \cdot \cos (k x) \cdot d k+\frac{U^{2}}{2}\right. & \\
& \left.+U^{2} R^{2} \int_{0}^{\infty} k \cdot \sin (k x)\left[e^{-k(z+f)}+\alpha(k) \cdot e^{k y}\right] \cdot d k\right\}=0
\end{aligned}
$$

Since this equality is valid for any point of the free surface, using the points with $z$ equals to zero, it is given that:

$$
g \cdot \beta(k)+k \cdot U^{2} R^{2} \cdot e^{-k f}-k \cdot U \cdot \alpha(k)=0
$$

Which is the same equation found in (Lamb, 1879B). This expression with the equation ( 6.18 ) leads to a linear system with two equations for solving $\alpha(k)$ and $\beta(k)$. T/he results are: 


$$
\begin{gathered}
\alpha(k)=\frac{k U^{2}+g}{k U^{2}-g} R^{2} \cdot U \cdot e^{-k f} \\
\beta(k)=2 \cdot \frac{R^{2} \cdot U^{2} \cdot k}{k U^{2}-g} \cdot e^{-k f}
\end{gathered}
$$

Hence, the free surface is given by the following integral:

$$
\eta(x)=\frac{2 \cdot R^{2} \cdot U^{2}}{g} \int_{0}^{\infty} \frac{1}{\left(k U^{2} / g\right)-1} \cdot k \cdot e^{-k f} \cdot \cos (k x) \cdot d k
$$

By the Buckingham Pi Theorem (Munson et. al, 2004), the problem could be dimensionless by:

$$
\eta^{*}=\frac{\eta}{R}=f_{\eta}\left(F r_{R}, \frac{x}{R}, \frac{f}{R}, k \cdot R\right)
$$

Substituting those values on the integral:

$$
\eta^{*}\left(x^{*}\right)=2 \cdot F_{R}^{2} \int_{0}^{\infty} \frac{1}{k^{*} F r_{R}^{2}-1} \cdot k^{*} \cdot e^{-k^{*} f^{*}} \cdot \cos \left(k^{*} x^{*}\right) \cdot d k^{*}
$$

For small Froude Numbers is expected an appearance of a disturbance with the wave length, $\lambda^{*}$, and amplitude, $\mathrm{H}^{*}$, equal to:

$$
\begin{gathered}
\lambda^{*}=2 \cdot \pi \cdot F r_{R}^{2} \\
\mathrm{H}^{*}=\frac{2 . \pi}{F r_{R}^{2}} \cdot e^{-k^{*} f^{*}}
\end{gathered}
$$

Hence, the shape of free surface is described by:

$$
\eta(x)=R \cdot \frac{2 \cdot \pi}{F r_{R}^{2}} \cdot \exp \left(-\frac{f}{R \cdot F r_{R}^{2}}\right) \cdot \sin \left(\frac{1}{F r_{R}^{2}} \cdot \frac{x}{R}\right)
$$




\subsubsection{Linear Wave Propagation}

The Figure 23 shows the domain used for analysing the Linear Wave propagation using the eastic wall approach and hence neglecting the gravity. The boundary conditions of the system are shown in, in which there is no net flux trough the free surface, there is no net flux trough the lower wall and the solid deformation in the lower portion is equal to the free surface deformation. The fluid and solid are coupled through the Extended Hamilton Principle (Meirovitch, 2001) which states that the variational of the action integral, $\mathcal{A}$, it is equal to zero, since this integral is stationary.

$$
\delta \mathcal{A}=\delta \int_{t_{1}}^{t_{2}}\left(\tilde{\mathcal{T}}-\tilde{\mathcal{V}}+\widetilde{\mathcal{W}}_{n c}\right) \cdot d t=0
$$

Where:

$\tilde{\mathcal{T}} \quad$ Is the kinetic energy;

$\tilde{\mathcal{V}} \quad$ Is the potential energy;

$\widetilde{\mathcal{W}}_{n c} \quad$ Work associated with non-conservative forces.

Since for this analysis the structural damping is neglected, and the fluid flow is potential, there is no non-conservative work. The kinetic energy is divided in two components, the first is due to solid movement and the second is due to the fluid movement. The potential energy happens due to solid deformation. Hence, the action integral is divided in three components. Applying the definitions of kinetic and potential energy for a continuous media according to (Lanczos, 1949):

Figure 23 - Elastic wall for modelling free surface in linear wave theory

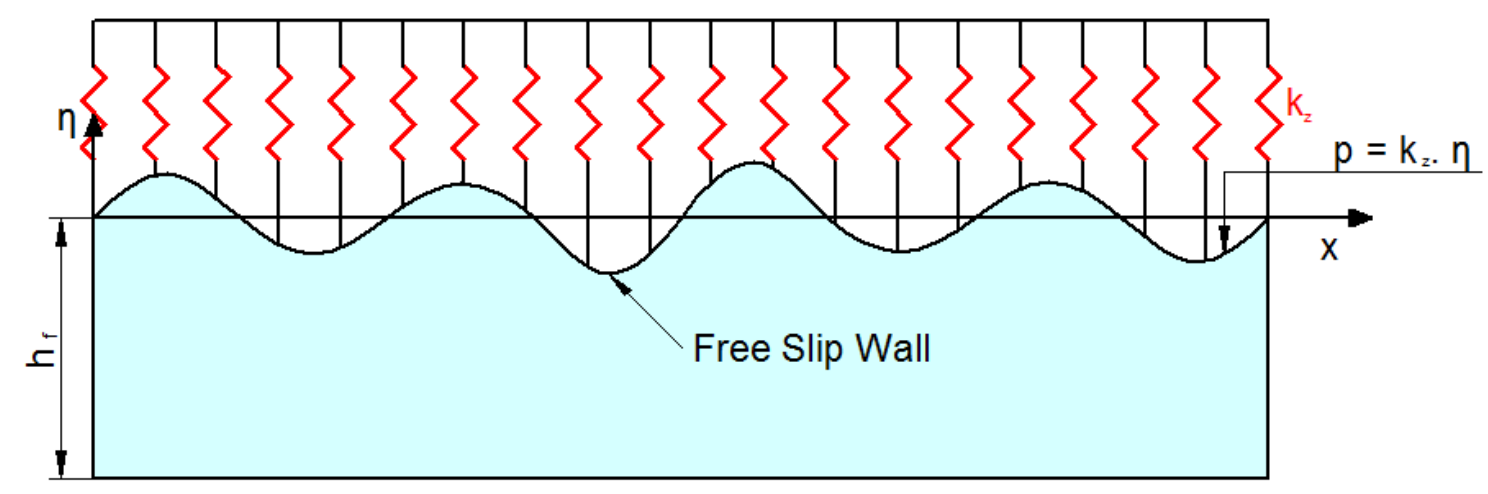




$$
\begin{gathered}
\mathcal{A}=\mathcal{A}_{K S}+\mathcal{A}_{K F}+\mathcal{A}_{P S} \\
\mathcal{A}_{K S}=\int_{t_{1}}^{t_{2}} \int_{0}^{L} \int_{0}^{h_{S}} \frac{\rho_{s}}{2}\left(\frac{\partial w_{s}}{\partial t}\right)^{2} d z \cdot d x \cdot d t \\
\mathcal{A}_{K F}=\int_{t_{1}}^{t_{2}} \int_{0}^{L} \int_{-h_{f}}^{\eta(x, t)} \frac{\rho_{f}}{2}\left(\mathrm{~V}_{f}\right)^{2} d z \cdot d x \cdot d t \\
\mathcal{A}_{P S}=-\int_{t_{1}}^{t_{2}} \int_{0}^{L} \int_{0}^{h_{s}} \frac{E_{z}}{2}\left(\frac{\partial w_{s}}{\partial z}\right)^{2} d z \cdot d x \cdot d t
\end{gathered}
$$

Where:

$\mathcal{A}_{K S} \quad$ Is the solid kinetic energy;

$\mathcal{A}_{K F} \quad$ Is the fluid kinetic energy;

$\mathcal{A}_{P S} \quad$ Is the solid potential energy;

$w_{S} \quad$ Is the solid vertical displacement;

$\mathrm{V}_{f} \quad$ Is the modulus of a fluid particle velocity vector;

$V_{f, x} \quad$ Is the horizontal component of a fluid particle velocity vector;

$V_{f, z} \quad$ Is the vertical component of a fluid particle velocity vector.

Applying the variational operator on the action integral due to solid kinetic energy, and performing the integration by parts with respect to the time:

$$
\begin{aligned}
\delta \mathcal{A}_{K S}=\int_{0}^{L} \int_{0}^{h_{S}} \int_{t_{1}}^{t_{2}} \rho_{s} \frac{\partial w_{s}}{\partial t} \delta\left(\frac{\partial w_{s}}{\partial t}\right) d t \cdot d z \cdot d x \\
=\int_{0}^{L} \int_{0}^{h_{S}}\left[\left.\rho_{s} \frac{\partial w_{s}}{\partial t} \delta w_{S}\right|_{t_{1}} ^{t_{2}}-\int_{t_{1}}^{t_{2}} \rho_{s} \frac{\partial^{2} w_{s}}{\partial t^{2}} \delta w_{s} d t \cdot\right] d z \cdot d x
\end{aligned}
$$

Since the variational is supposed to be known in the instants $t_{1}$ and $t_{2}$, rearranging the integral, the term $\delta \mathcal{A}_{K S}$ becomes:

$$
\delta \mathcal{A}_{K S}=\int_{t_{1}}^{t_{2}} \int_{0}^{L} \int_{0}^{h_{S}}-\rho_{s} \frac{\partial^{2} w_{s}}{\partial t^{2}} \delta w_{s} d z \cdot d x \cdot d t
$$


Applying the variational operator on the action integral due to solid potential energy, and performing the integration by parts with respect to the $z$ axis:

$$
\begin{aligned}
\delta \mathcal{A}_{P S}=-\int_{t_{1}}^{t_{2}} \int_{0}^{L} \int_{0}^{h_{S}} E_{z} \frac{\partial w_{s}}{\partial z} \delta\left(\frac{\partial w_{s}}{\partial z}\right) d z \cdot d x \cdot d t \\
=\int_{t_{1}}^{t_{2}} \int_{0}^{L}\left[-\left.E_{z} \frac{\partial w_{s}}{\partial z} \delta w_{s}\right|_{0} ^{h_{S}}+\int_{0}^{h_{S}} E_{z} \frac{\partial^{2} w_{s}}{\partial z^{2}} \delta w_{s} d t .\right] d z \cdot d x
\end{aligned}
$$

Since the variational of solid displacement is equal to zero on the fixed surface, $z=h_{s}$ and in the lower face, the virtual displacement is equal to the free surface deformation, it is given that:

$$
\delta \mathcal{A}_{P S}=\int_{t_{1}}^{t_{2}} \int_{0}^{L}\left[E_{z} \frac{\partial w_{s}}{\partial z} \delta \eta+\int_{0}^{h_{S}} E_{z} \frac{\partial^{2} w_{s}}{\partial z^{2}} \delta w_{s} d z\right] \cdot d x \cdot d t
$$

The approach for the action integral due to fluid kinetic energy is different since the fluid domain is deformable with respect to the integration limits. According to the procedure described in (Gelfand \& Fomin, 1963), in which the variational of a functional $\mathcal{J}$, given by the integral of a function $F_{\mathcal{J}}$ between the limits $a(t)$ and $b(t)$ is given by:

$$
\delta \mathcal{J}=\delta \int_{a(t)}^{b(t)} F_{\mathcal{J}} \cdot d t=F_{\mathcal{J}}(\delta b-\delta a)+\int_{a(t)}^{b(t)} \delta\left(F_{\mathcal{J}}\right) \cdot d t
$$

Hence, applying this approach for the fluid domain is given that:

$$
\delta \mathcal{A}_{K F}=\int_{t_{1}}^{t_{2}} \int_{0}^{L}\left[\frac{\rho_{f}}{2}\left(\mathrm{~V}_{f}\right)^{2} \delta \eta+\int_{-h_{f}}^{\eta(x, t)} \frac{\rho_{f}}{2} \delta\left[\left(\mathrm{V}_{f}\right)^{2}\right] \cdot d z\right] \cdot d x \cdot d t
$$

From the chain rule and assuming that the fluid velocity is equal to the derivative of the position vector with respect to time, it is given that:

$$
\delta \mathcal{A}_{K F}=\int_{t_{1}}^{t_{2}} \int_{0}^{L} \frac{\rho_{f}}{2}\left(\mathrm{~V}_{f}\right)^{2} \delta \eta \cdot d x \cdot d t+\int_{t_{1}}^{t_{2}} \int_{0}^{L} \int_{-h_{f}}^{\eta(x, t)} \rho_{f} \overrightarrow{\mathrm{V}_{f}} \delta\left(\frac{d \overrightarrow{\mathrm{x}}}{d t}\right) \cdot d z \cdot d x \cdot d t
$$


Analysing the second term from right hand of the previous equation, and integrating by parts with respect to time:

$$
\int_{t_{1}}^{t_{2}} \int_{0}^{L} \int_{-h_{f}}^{\eta(x, t)} \rho_{f} \overrightarrow{\mathrm{V}_{f}} \delta\left(\frac{d \overrightarrow{\mathrm{x}}}{d t}\right) \cdot d z \cdot d x \cdot d t=\rho_{f} \int_{0}^{L} \int_{-h_{f}}^{\eta(x, t)}\left[\left.\overrightarrow{\mathrm{V}_{f}} \delta \overrightarrow{\mathrm{x}}\right|_{t_{1}} ^{t_{2}}-\int_{t_{1}}^{t_{2}} \frac{d \overrightarrow{\mathrm{V}_{f}}}{d t} \delta \overrightarrow{\mathrm{x}} \cdot d t\right] d z \cdot d x
$$

Using now an Eulerian description of the velocity frame and assuming known the position of the particles in the instants of time $t_{1}$ and $t_{2}$, and assuming a potential fluid flow in which the velocity potential is given by $\phi_{W}$ :

$$
\begin{aligned}
\int_{t_{1}}^{t_{2}} \int_{0}^{L} \int_{-h_{f}}^{L(x, t)} \rho_{f} & \overrightarrow{\mathrm{V}_{f}} \delta\left(\frac{d \overrightarrow{\mathrm{x}}}{d t}\right) \cdot d z \cdot d x \cdot d t \\
& =-\rho_{f} \int_{t_{1}}^{t_{2}} \int_{0}^{L} \int_{-h_{f}}^{\eta(x, t)} \frac{\partial}{\partial t}\left(\nabla \phi_{W}\right)+\nabla\left[\frac{\left(\nabla \phi_{W}\right)^{2}}{2}\right] \cdot \delta \overrightarrow{\mathrm{x}} \cdot d z \cdot d x \cdot d t
\end{aligned}
$$

Performing the dot product between the gradient of the derivative of $\phi_{W}$ with respect to time and the $\delta \overrightarrow{\mathrm{x}}$ :

$$
\begin{aligned}
\int_{t_{1}}^{t_{2}} \int_{0}^{L} \int_{-h_{f}}^{L(x, t)} \rho_{f} \overrightarrow{\mathrm{V}_{f}} \delta & \left(\frac{d \overrightarrow{\mathrm{x}}}{d t}\right) \cdot d z \cdot d x \cdot d t \\
& =-\rho_{f} \int_{t_{1}}^{t_{2}} \int_{0}^{L} \int_{-h_{f}}^{\eta(x, t)}\left(\frac{\partial}{\partial x} \frac{\partial \phi_{W}}{\partial t} \delta x+\frac{\partial}{\partial z} \frac{\partial \phi_{W}}{\partial t} \delta z\right) \\
& +\nabla\left[\frac{\left(\nabla \phi_{W}\right)^{2}}{2}\right] \cdot \delta \overrightarrow{\mathrm{x}} \cdot d z \cdot d x \cdot d t
\end{aligned}
$$

Integrating $\frac{\partial}{\partial x} \frac{\partial \phi_{W}}{\partial t} \delta x$ with respect to $x$ and assuming known the variable $x$ in 0 and $L$ and integrating $\frac{\partial}{\partial z} \frac{\partial \phi_{W}}{\partial t} \delta z$ with respect to $z, x$ and assuming known the variable $z$ in 0 and in $z=\eta(x, t)$ the $z$ position is equal to $\eta(x, t)$ is given that:

$$
\begin{aligned}
\int_{t_{1}}^{t_{2}} \int_{0}^{L} \int_{-h_{f}}^{\eta(x, t)} \rho_{f} \overrightarrow{\mathrm{V}_{f}} \delta & \left(\frac{d \overrightarrow{\mathrm{x}}}{d t}\right) \cdot d z \cdot d x \cdot d t \\
& =-\rho_{f} \int_{t_{1}}^{t_{2}} \int_{0}^{L}\left[\frac{\partial \phi_{W}}{\partial t} \delta \eta+\int_{-h_{f}}^{\eta(x, t)} \nabla\left[\frac{\left(\nabla \phi_{W}\right)^{2}}{2}\right] \cdot \delta \overrightarrow{\mathrm{x}} \cdot d z \cdot\right] d x \cdot d t
\end{aligned}
$$


The last term could be rearranged in: $\nabla\left[\frac{\left(\nabla \phi_{W}\right)^{2}}{2}\right] \cdot \delta \overrightarrow{\mathrm{x}}=\nabla^{2} \phi_{W} \delta \phi_{W}$. Hence:

$$
\delta \mathcal{A}_{K F}=\int_{t_{1}}^{t_{2}} \int_{0}^{L}\left\{\left[\frac{\rho_{f}}{2}\left(\nabla \phi_{W}\right)^{2}+\frac{\partial \phi_{W}}{\partial t}\right] \delta \eta+\int_{-h_{f}}^{\eta(x, t)} \nabla^{2} \phi_{W} \delta \phi_{W} \cdot d z\right\} \cdot d x \cdot d t
$$

Putting all equations together:

$$
\begin{gathered}
\delta \mathcal{A}=\int_{t_{1}}^{t_{2}} \int_{0}^{L}\left\{\left[\frac{\rho_{f}}{2}\left(\nabla \phi_{W}\right)^{2}+\frac{\partial \phi_{W}}{\partial t}+E_{z} \frac{\partial w_{s}}{\partial z}\right] \delta \eta+\int_{-h_{f}}^{\eta(x, t)} \nabla^{2} \phi_{W} \delta \phi_{W} \cdot d z\right. \\
\left.-\int_{0}^{h_{s}}\left[\rho_{s} \frac{\partial^{2} w_{s}}{\partial t^{2}}-E_{z} \frac{\partial^{2} w_{s}}{\partial z^{2}}\right] \delta w_{s} d z\right\} \cdot d x \cdot d t=0
\end{gathered}
$$

For guarantee that the integral is equal to zero for any virtual displacement of the quantities $w_{s}, \phi_{W}$ and $\eta$, it is given that:

$$
\begin{gathered}
\rho_{s} \frac{\partial^{2} w_{s}}{\partial t^{2}}-E_{z} \frac{\partial^{2} w_{s}}{\partial z^{2}}=0 \\
\left.\rho_{f} \frac{\partial \phi_{w}}{\partial t}\right|_{z=\eta}+\left.\frac{\rho_{f}}{2}\left(\nabla \phi_{w}\right)^{2}\right|_{z=\eta}+\left.E_{z} \frac{\partial w_{s}}{\partial z}\right|_{z=h_{s}}=0 \\
\nabla^{2} \phi_{W}=0
\end{gathered}
$$

The equation ( 6.53 ) represents the equation for the longitudinal vibration of a bar. The natural frequencies depend on the boundary conditions and this problem is explored on (Rao, 2008). The equation (6.53).b represents the Bernoulli equation on the free surface in which the potential energy due to gravity is substituted by the energy due to solid strain. The equation (6.53). $c$ is the Laplace, equation for the fluid which is consequence from the assumption of potential fluid flow.

Admitting that the solid density is negligible, the equation ( 6.53 ) becomes:

$$
\frac{\partial^{2} w_{s}}{\partial z^{2}}=0 \rightarrow w_{s}(x, z, t)=A_{s}+B_{s} \cdot z
$$


Since the upper face of the solid is modeled as fixed, the coefficient $A_{s}$ is equal to zero. Hence, the first derivative of $w_{s}$ is constant with respect to $z$. Hence, if the lower face deformation is equal to $\eta$, the strain is given by:

$$
\frac{\partial w_{s}}{\partial z}=\frac{\eta}{h_{s}}
$$

From the definition of $E_{z}(6.13)$, substituting the result of equation ( 6.57 ), in the equation (8.54), it is derived the following equation:

$$
\left.\rho_{f} \frac{\partial \phi_{w}}{\partial t}\right|_{z=\eta}+\left.\frac{\rho_{f}}{2}\left(\nabla \phi_{w}\right)^{2}\right|_{z=\eta}+\rho_{f} \cdot g \cdot \eta=0
$$

Deriving the last equation with respect to time and rearranging the terms:

$$
\rho_{f} g \frac{\partial \eta}{\partial t}=-\left.\rho_{f} \frac{\partial^{2} \phi_{w}}{\partial t^{2}}\right|_{z=\eta}-\rho_{f}\left[\frac{\partial \phi_{w}}{\partial x} \overrightarrow{e_{x}}+\frac{\partial \phi_{w}}{\partial z} \overrightarrow{e_{z}}\right] \cdot\left[\frac{\partial}{\partial x} \overrightarrow{e_{x}}+\frac{\partial}{\partial z} \overrightarrow{e_{z}}\right] \frac{\partial \phi_{w}}{\partial t}
$$

This equation is the same as (A.2.7), derived for linear gravity waves in the Attachment A.2. Therefore, it is concluded that is possible to achieve the same results for linear gravity waves propagation by neglecting the gravity effects and assuming an elastic wall in the free surface. However, mathematical models and simulations for transient effects considering the solid density still required for full validation of this model. 


\subsubsection{Actuator Disk Model for an Open Channel}

The differences between actuator disk in an open channel, derived on Attachment, are with respect to the Bernoulli equation and momentum conservation equation since the pressure distribution is affected in the domain boundaries while the equations derived from continuity equation are maintained.

Using the pressure distribution shown in the Figure 24 it is derived the total force due to pressure in control volume. The contributions of inlet, outlet and free surface must be taken in account. Hence, the total force due to the pressure in the control volume is given by:

$$
\mathrm{F}_{\mathrm{x}}=\mathrm{F}_{\mathrm{x}, \text { inlet }}-\mathrm{F}_{\mathrm{x}, \text { outlet }}+\mathrm{F}_{\mathrm{x}, \mathrm{FS}}
$$

In the free surface:

$$
\mathrm{F}_{\mathrm{x}, \mathrm{FS}}=\mathrm{b} \int_{\eta}-\nabla \mathrm{V}_{\mathrm{EFS}} \mathrm{d} \overrightarrow{\mathrm{l}}
$$

Since the line integral applied to an gradient is independent from the path, it is given that:

$$
\mathrm{F}_{\mathrm{x}, \mathrm{FS}}=\mathrm{b}\left[\mathrm{V}_{\mathrm{EFS}}(0)-\mathrm{V}_{\mathrm{EFS}}(\mathrm{L})\right]=-\frac{\rho \cdot \mathrm{g} \cdot \mathrm{b}}{2}\left[2 \cdot \mathrm{h} \cdot \Delta \mathrm{h}-\frac{(\Delta \mathrm{h})^{2}}{2}\right]
$$

The components from inlet and outlet are given by the multiplying the pressure by the inlet and outlet areas, according to the following equation:

$$
F_{x, \text { inlet }}-F_{x, \text { outlet }}=b\left[\int_{-h}^{0} p_{i} \cdot d z-\int_{-h}^{-\Delta h} p_{o} \cdot d z\right]=p_{i} A_{i}-p_{o} A_{o}
$$

Developing the equation is given that:

$$
F_{x, \text { inlet }}-F_{x, \text { outlet }}=\frac{\rho \cdot g \cdot b}{2}\left[4 . h \cdot \Delta h-2 .(\Delta h)^{2}\right]
$$

Since the pressure distribution and the free surface are not known a priori, a different approach will be used. The elastic free surface model could be derived by a potential function: 


$$
p \cdot \vec{n}=-\nabla \mathcal{V}_{E F S}
$$

This potential function is given by:

$$
\mathcal{V}_{E F S}=-\rho \cdot g \cdot\left(\eta+\frac{\eta^{2}}{2}\right)
$$

Therefore, the pressure force on the free surface is given by:

$$
p \cdot \vec{n}=\rho \cdot g \cdot(1+\eta)\left(\frac{\partial \eta}{\partial x} \overrightarrow{e_{x}}+\overrightarrow{e_{z}}\right)
$$

By this methodology, the force on $x$ direction on the free surface is given by:

$$
F_{x, F S}=b \int_{\eta} p \cdot \vec{n} \cdot \overrightarrow{e_{x}} \cdot d \eta=b \int_{\eta} \rho \cdot g \cdot(1+\eta) \cdot \frac{\partial \eta}{\partial x} \cdot d \eta
$$

However, if the term $d \eta$ is substituted by $d \vec{l}=\frac{\partial \eta}{\partial x} \cdot \overrightarrow{e_{x}} \cdot d x$, it is given the following expression for the force due to pressure on free surface, in $x$ direction:

$$
F_{x, F S}=b \int_{\eta}-\nabla \mathcal{V}_{E F S} d \vec{l}
$$

Since the line integral of gradient is independent from the path, it is given that:

$$
F_{x, F S}=b\left[\mathcal{V}_{E F S}(0)-\mathcal{V}_{E F S}(L)\right]=-\frac{\rho \cdot g \cdot b}{2}\left[2 \cdot h \cdot \Delta h-\frac{(\Delta h)^{2}}{2}\right]
$$

The total force due to pressure in control volume is given by:

$$
F_{x}=F_{x, \text { inlet }}-F_{x, \text { outlet }}+F_{x, F S}
$$

Combining the equations (6.60), ( 6.66 ) and (6.67):

$$
F_{x}=\frac{\rho \cdot g \cdot b}{2}\left[2 \cdot h \cdot \Delta h-(\Delta h)^{2}\right]
$$


Figure 24 - Pressure distribution due to the elastic free surface

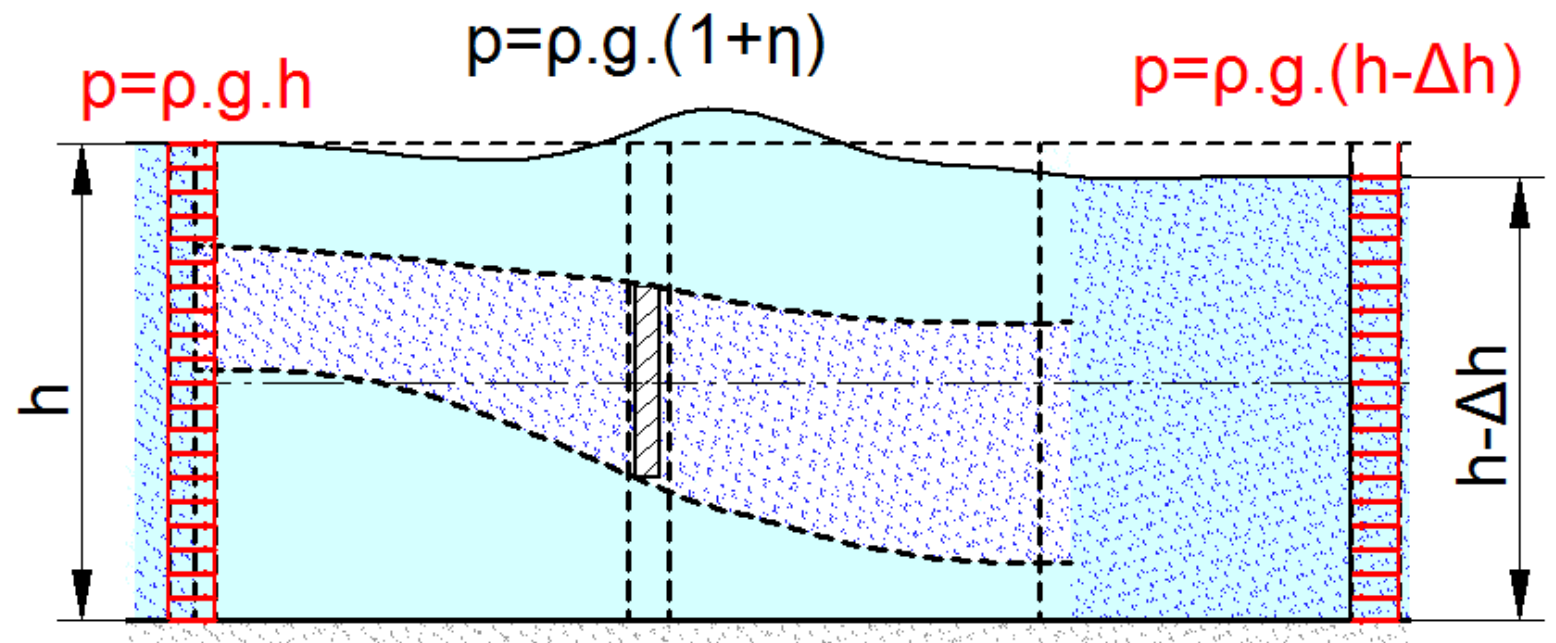

The other difference related to the pressure distribution is with respect to the Bernoulli equation in the free surface. Applying this equation between the stations "I" and "ob" in by-pass flow is given that:

$$
\rho \cdot \frac{u^{2}}{2}+\rho g h=\rho \cdot \frac{\alpha_{o b, b}^{2} \cdot u^{2}}{2}+\rho \cdot g(h-\Delta h)
$$

This equation leads to:

$$
\Delta h=\frac{u^{2}}{2 g}\left(1-\alpha_{o b, b}^{2}\right)
$$

By applying the Bernoulli equation between inlet section and outlet before mixing section in the turbine region, neglecting the buoyancy and applying the modified pressure distribution on the free surface, it is given the same results from the equation (C.26). Hence, since all equations are the same, even with different considerations and one model completely neglecting the gravity effects, the results are expected to be the same with respect to hydrodynamic performance prediction by the actuator disk model for an open channel.

Besides of that, in (Mattavo \& Assi, 2017), it is highlighted that the only difference in the forces in an immersed body subjected to a confined flow by a free surface, as the case of a tidal current turbine, it is the buoyancy. Thus, in the streamwise direction, the results are expected to be the same. However, since the buoyancy force is easy to be calculated, this effect could be neglected in the analysis. 


\subsection{Steady State Finite Element Model from Elastic Free Surface}

The orthotropic properties of the solid are calculated according the procedure of section 6.1.1. in the Table 2. The solid was modeled with the height equivalent to 3 times of the fluid domain.

Additionally, are calculated the first natural mode in vertical direction even no transient analysis being performed. This frequency is calculated assuming a rod with a fixed end, since the solid is modeled for no shear transmission. With this result is calculated the gravity wave length with this same frequency to compare the mesh size with this length. Since frequency and length are inversely proportional, according to the linear wave theory and the dispersion relation, if the maximum wave length which is associated with the first natural frequency is lower than the mesh size, it is expected that no resonance effect will occur in a transient analysis.

At first moment, the solid density is adjusted for the same density as atmospheric air. However, for the present work, this value will not make any difference on results since the performed analysis is in the steady state. The mechanical properties are summarised on the Table 3.

Since the solution provided by finite element method is discretized, a mesh convergence analysis is performed. However, since the model is focused on analysing deflections, a coarse mesh is expected to present reasonable results. For stress concentration for example, in which deformation gradients must be calculated with precision, a higher mesh refinement is required.

Table 3 - Elastic Solid Mechanical Properties

\begin{tabular}{|l|c|c|c|}
\hline Property & Symbol & Value & Unit \\
\hline Solid Density & $\rho$ & 1.225 & $\mathrm{~kg} / \mathrm{m}^{3}$ \\
\hline Elasticity Modulus & $E$ & $3.52 \times 10^{-2}$ & $\mathrm{MPa}$ \\
\hline Shear Modulus & $G$ & $3.52 \times 10^{-5}$ & $\mathrm{MPa}$ \\
\hline Poisson Coefficient & $v$ & $1 \times 10^{-3}$ & {$[-]$} \\
\hline Solid Pre-Stress & $p_{a}$ & $1.17 \times 10^{-2}$ & $\mathrm{MPa}$ \\
\hline First Axial Natural Mode & $\omega_{0}$ & 74.0 & $\mathrm{rad} / \mathrm{s}$ \\
\hline Gravity wave length & $\lambda_{0}$ & 11.2 & $\mathrm{~mm}$ \\
\hline
\end{tabular}


Figure 25 - Mesh used for Orthotropic Solid Finite Element Model
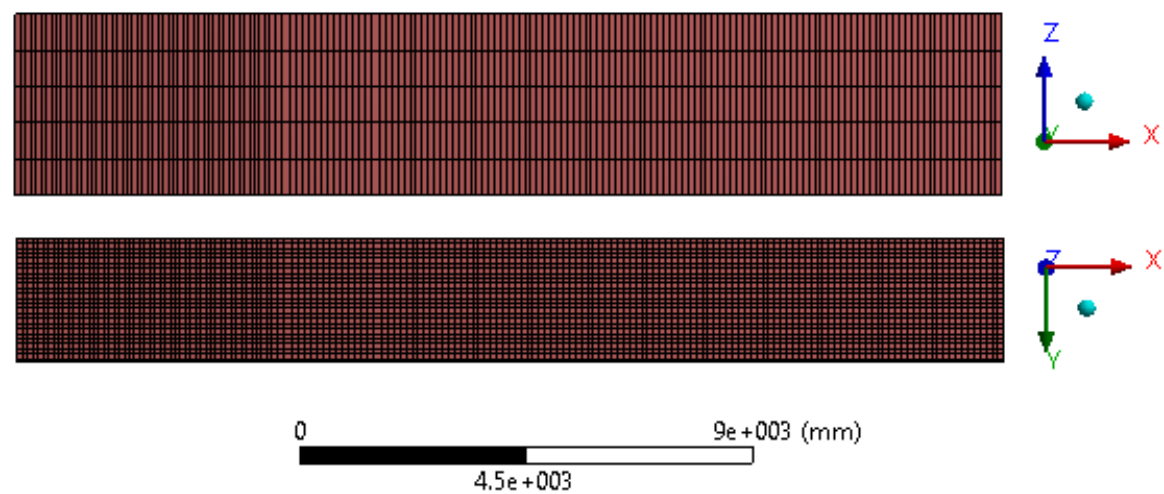

The mesh convergence analysis is performed with the results provided by the fluid model from confined domain (sections 7.3and 8.3. The results from TSR $=6$ were used. The choice for these loads for mesh analysis is that the model must be sufficiently refined to deform reacting to the fluid flow pressure. Hence, the finite element model is validated against the real loading condition.

According to the Table 4, the change in number of divisions does not affect substantially the total displacement, hence, mesh with 5 divisions in height direction, 24 in the width direction and 170 divisions in length direction was chosen providing a mesh has 97149 nodes and 20400 elements. The frontal and inferior views from this mesh are shown in the Figure 25.

Table 4 - Orthotropic Solid Finite Element Model Mesh Convergence Analysis

\begin{tabular}{|c|c|c|c|c|c|c|}
\hline \multicolumn{5}{|c|}{ Number of Divisions [-] } & \multicolumn{2}{c|}{ Displacement [mm] } \\
\hline Height & Width & Upstream L. & Turbine L. & Downstream L. & Maximum & Minimum \\
\hline 2 & 24 & 50 & 20 & 100 & 1.241 & 31.52 \\
\hline 5 & 24 & 50 & 20 & 100 & 1.238 & 31.51 \\
\hline 10 & 24 & 50 & 20 & 100 & 1.231 & 31.48 \\
\hline 2 & 48 & 50 & 20 & 100 & 1.265 & 31.66 \\
\hline 5 & 48 & 50 & 20 & 100 & 1.264 & 31.65 \\
\hline 10 & 48 & 50 & 20 & 100 & 1.261 & 31.62 \\
\hline 2 & 24 & 100 & 40 & 200 & 1.265 & 31.66 \\
\hline 5 & 24 & 100 & 40 & 200 & 1.264 & 31.65 \\
\hline 10 & 24 & 100 & 40 & 200 & 1.268 & 31.68 \\
\hline 2 & 48 & 100 & 40 & 200 & 1.261 & 31.62 \\
\hline 5 & 48 & 100 & 40 & 200 & 1.261 & 31.62 \\
\hline 10 & 48 & 100 & 40 & 200 & 1.312 & 31.80 \\
\hline
\end{tabular}


Figure 26 - Fixed Support on Orthotropic Solid Upper Face

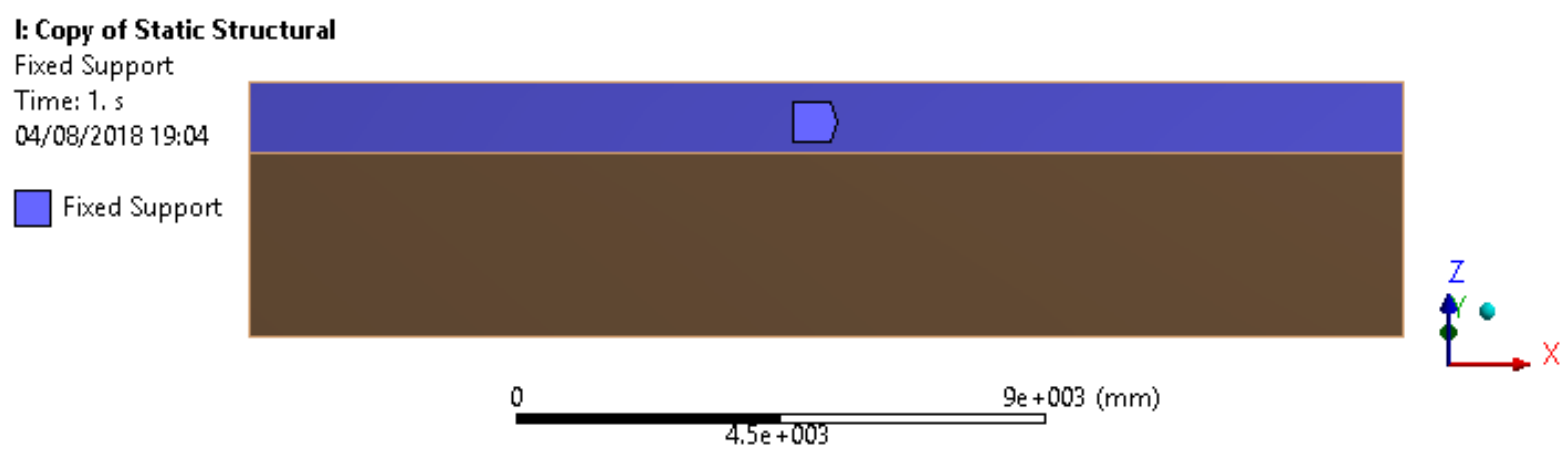

The upper face of the solid is modeled as fixed support. This boundary condition provides a zero displacement for all nodes included on this surface. This condition is presented in the Figure 26.

There are no boundary conditions defined for the lateral surfaces. The solid is free to deform in both $\mathrm{x}$ and $\mathrm{y}$ directions. Even that the theory was developed for plane state of deformation, the small values of the Poisson Coefficient guarantee that the deformation in those directions are negligible, therefore this model is physically consistent with the theory. The absence of boundary conditions on this region states that there are no stresses acting on this faces, hence, the gradient of deformation is equal to zero on those regions.

For the lower face, are considered three different boundary conditions and these boundary conditions are implemented separately in the domain, in the upstream region, turbine region and downstream region. The boundary conditions are: Filters, Presented in the Figure 27, Pre-stress, presented in the Figure 28 and Fluid Pressure, presented in the Figure 29.

Figure 27 - Displacement Filters on Orthotropic Solid Lower Face
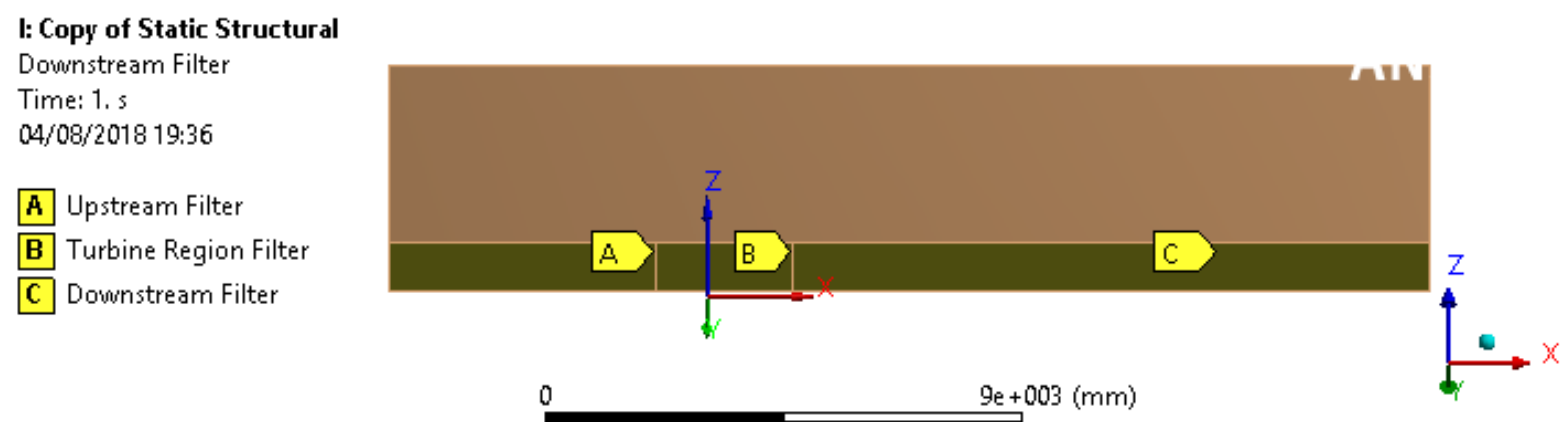
Figure 28 - Prestress on Orthotropic Solid Lower Face

I: Copy of Static Structural

Downstream Pressure

Time: 1.s

$04 / 08 / 201819: 38$

A Upstream Pressure: $-1.1763 \mathrm{e}-002 \mathrm{MP}$

B Turbine Region Pressure: $-1.1763 \mathrm{e}-$

C Downstream Pressure: $-1.1763 \mathrm{e}-002 \mathrm{MPa}$

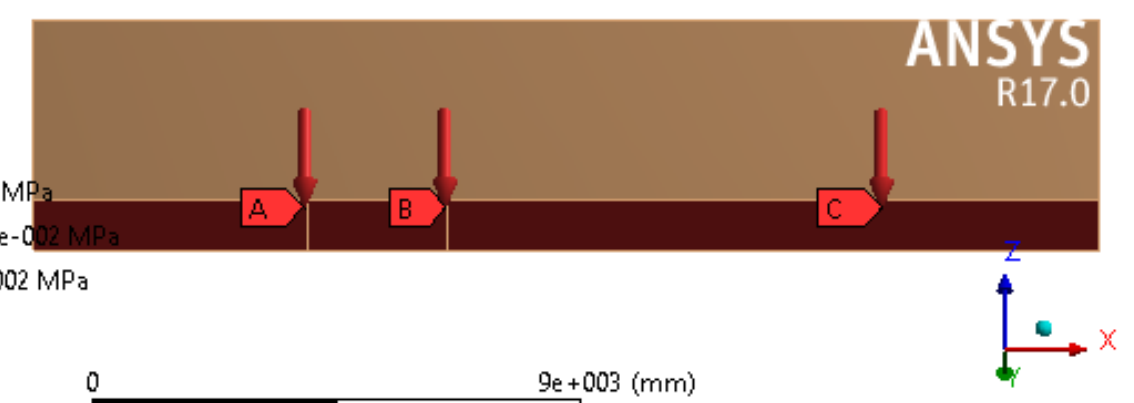

$4.5 e+003$

The filters are conditions for zero displacement in $x$ and $y$ directions. Due to the nature of the transference of force between fluid and solid, numerical errors may occurs and generate small forces in $\mathrm{x}$ and $\mathrm{y}$ directions, mainly for posterior iterations, in which the fluid face normal direction is no more parallel to $z$. Hence, this boundary condition guarantees that only forces in $z$ direction will be applied.

The pre-stress is a constant pressure applied according to the actuator disk model. According to this theory, this value is fundamental for the accuracy of the forces in the streamwise direction in the immersed body.

The Fluid-Solid interface is the input from the CFD simulation for the static structural simulation. In the commercial CFD code, the pressure is read in the fluid free surface and then exported for the Static Structural model and then applied as shown in the Figure 29.

Figure 29 - Fluid-Solid Interface on Orthotropic Solid Lower Face

I: Copy of Static Structural

Downstream Free Surface

Time: $1 . s$

04/08/201819:39

A Upstream Free Surface

B Turbine Region Free Surface

C Downstream Free Surface
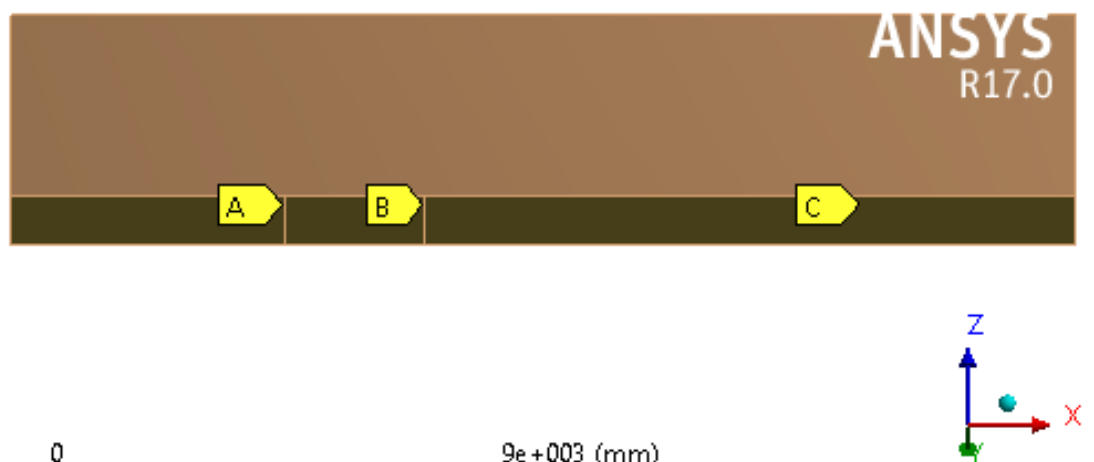


\section{Chapter 7. CFD Simulations}

This section is destined to presents the boundary conditions and central hypothesis used for the computational fluid dynamics simulations. All computational simulations consist in full modelling of turbine blades and hub, as shown in Figure 30 and the differences in the domain, studying the interference of each boundary condition on the flow pattern and in the power and thrust generated on turbine blades. This part is divided on a general overview, which includes general parameters, used for all simulations and open, confined and open channel domains, explaining each difference between the simulations. The divisions comprehend the following topics:

- General Overview

- General Mesh Parameters

- Mesh Interfaces

- Inlet Turbulence

- Turbine Walls Modelling

- Open Domain

- Opening Outlet

- External Diameter Boundary Conditions

- Domain Main Dimensions

- Confined Domain

- Opening Outlet

- External Walls Boundary Condition

- Domain Main Dimensions

- Open Channel Domain

- Opening Outlet

- Open Channel Walls

- Open Channel Free Surface

- Domain Main Dimensions 
Figure 30 - 3-D Model used for CFD Analysis

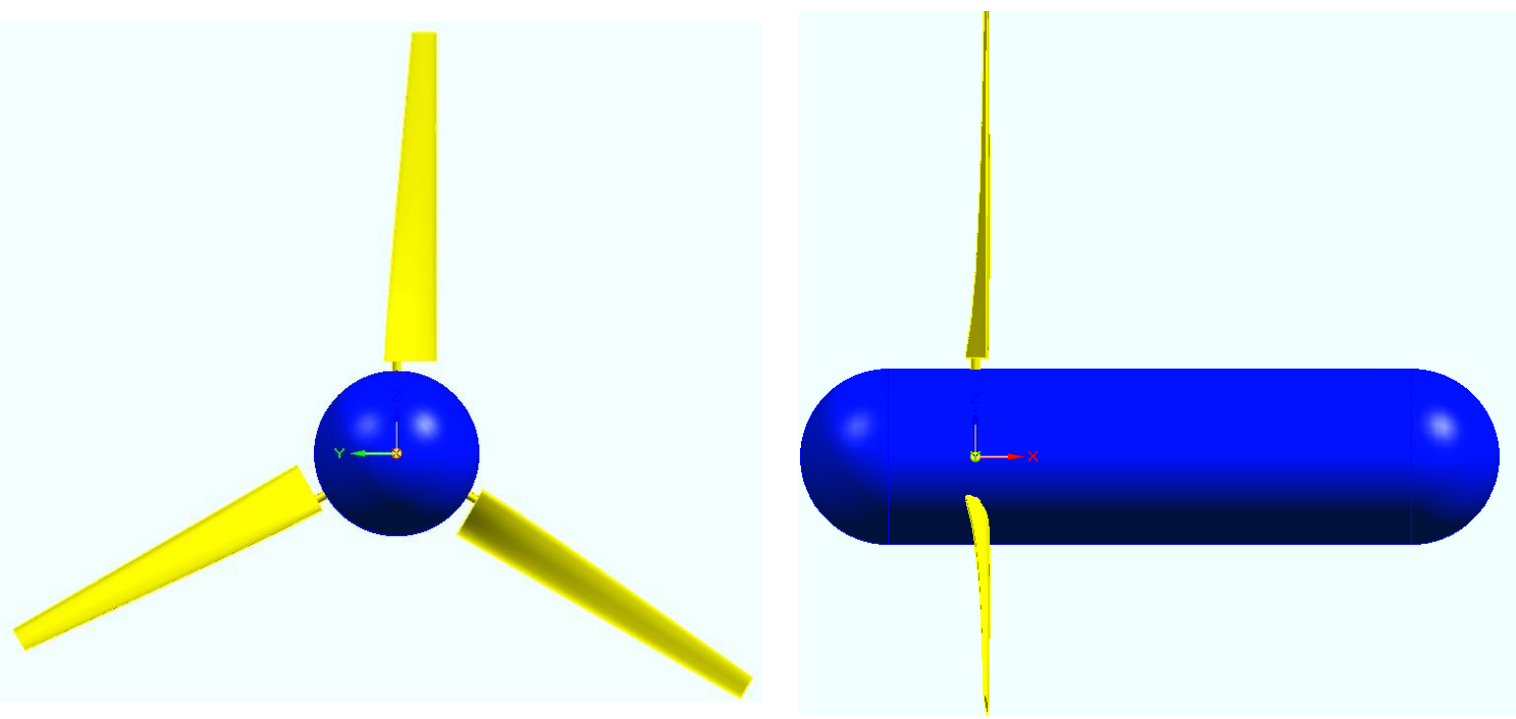

\subsection{General Overview}

All simulations are based on the same turbine geometry and pitch angle using a steady state approach. All analyses have the same mesh density on the rotating domain region, and the interfaces between rotating and static domain are maintained. Therefore, those parts are detailed in this section.

The coupling between velocity and pressure, shown in Figure 31 is used for all boundary conditions. It is destined to a collocated grid, as described in the section 4.4.1.

Figure 31 - Interpolation Scheme and Velocity-Pressure Coupling

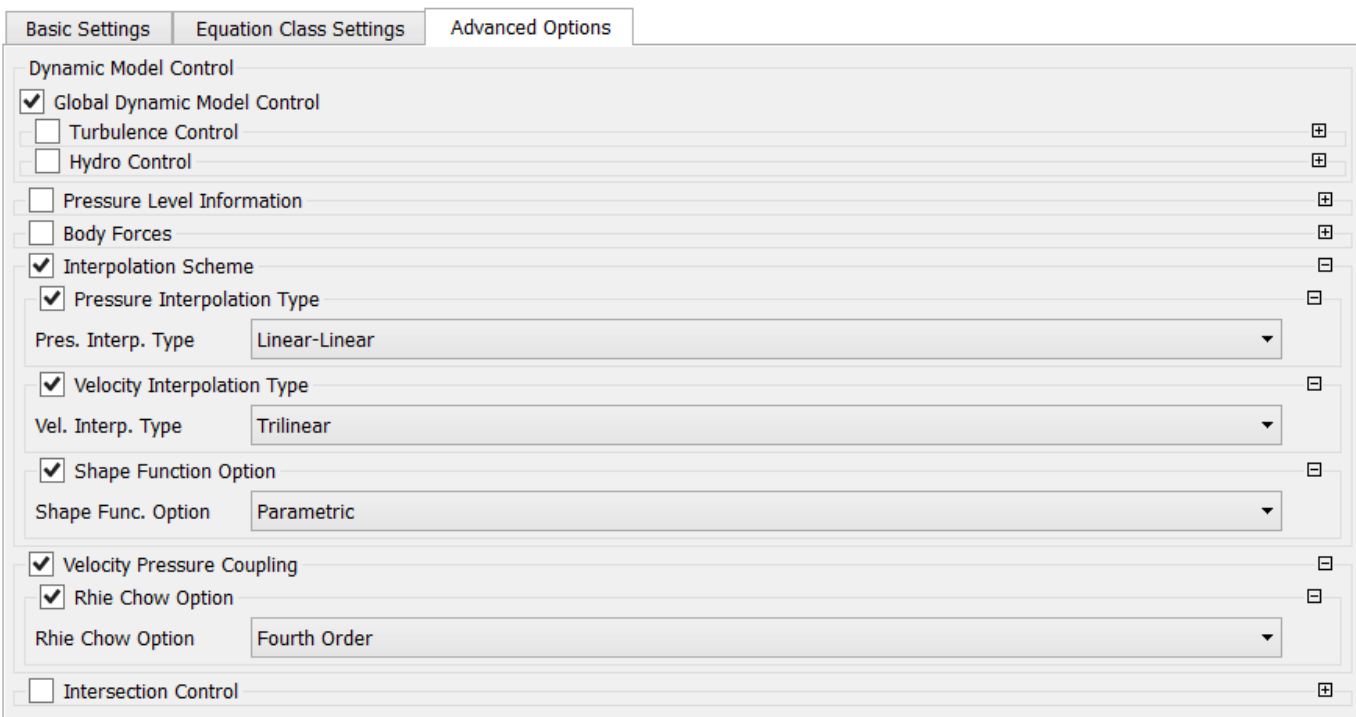


For implementing the rotational effects, the Moving Reference Frame (MRF) is implemented using a conservative interface for all transported variables. This model simulates the rotation through a source term that included the non-inertial accelerations and the no-slip condition in the walls assumes that all walls rotate about a fixed axis.

The geometry was constructed from (Bahaj, W.M.J., \& McCann, 2007), based on NACA 63-8XX series, interpolated from 17 sections in which the thickness, chord and pitch distribution along the radius are presented on the table below. The pitch angle presented in the table are considering a zero angle of twist in the blade. For this work, an additional angle of $20^{\circ}$ was added to each blade section since that this is the angle that achieved the highest power coefficient values during the experiments.

Table 5 - Turbine geometry

\begin{tabular}{|c|c|c|c|c|}
\hline$r / R[-]$ & $R[\mathrm{~mm}]$ & $c / R[-]$ & $\beta_{P}[\mathrm{deg}]$ & $t / c[\%]$ \\
\hline 0.20 & 80 & 0.1250 & 15 & 24 \\
\hline 0.25 & 100 & 0.1203 & 12.1 & 22.5 \\
\hline 0.30 & 120 & 0.1156 & 9.5 & 20.7 \\
\hline 0.35 & 140 & 0.1109 & 7.6 & 19.5 \\
\hline 0.40 & 160 & 0.1063 & 6.1 & 18.7 \\
\hline 0.45 & 180 & 0.1016 & 4.9 & 18.1 \\
\hline 0.50 & 200 & 0.0969 & 3.9 & 17.6 \\
\hline 0.55 & 220 & 0.0922 & 3.1 & 17.1 \\
\hline 0.60 & 240 & 0.0875 & 2.4 & 16.6 \\
\hline 0.65 & 260 & 0.0828 & 1.9 & 16.1 \\
\hline 0.70 & 280 & 0.0781 & 1.5 & 15.6 \\
\hline 0.75 & 300 & 0.0734 & 1.2 & 15.1 \\
\hline 0.80 & 320 & 0.0688 & 0.9 & 14.6 \\
\hline 0.85 & 340 & 0.0641 & 0.6 & 14.1 \\
\hline 0.90 & 360 & 0.0594 & 0.4 & 13.6 \\
\hline 0.95 & 380 & 0.0547 & 0.2 & 13.1 \\
\hline 1.00 & 400 & 0.0500 & 0.0 & 12.6 \\
\hline
\end{tabular}




\subsubsection{Rotating Domain Mesh}

Figure 32 - Rotating domain mesh
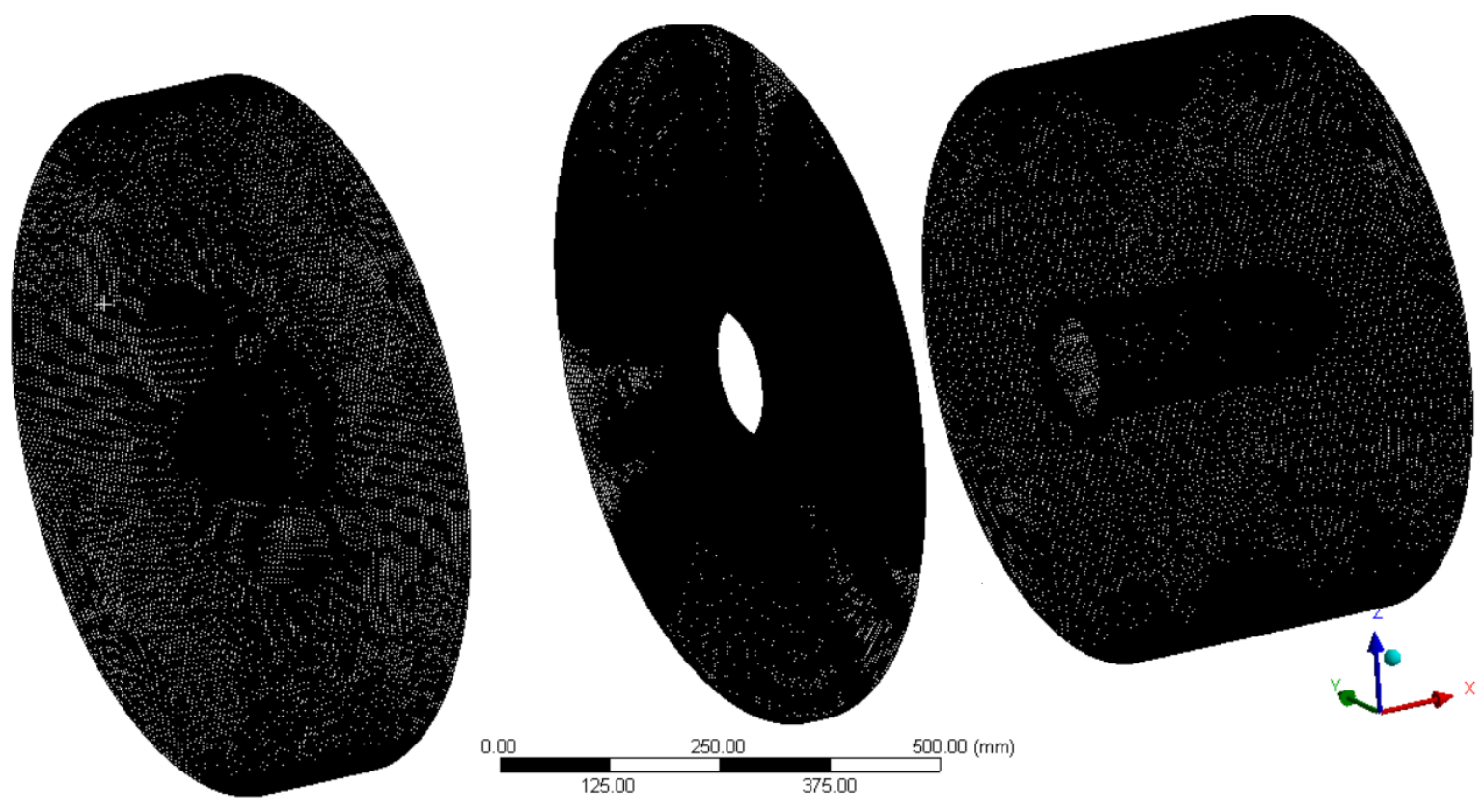

The Figure 32 shows a general overview of the mesh used in the rotating domain. For upstream and downstream hubs, a hexahedral unstructured mesh was used. The turbine disk region was divided into two parts: One close to the blades, that is shown in Figure 33, in which a hexahedral unstructured mesh was used and other between two blades in which a structured mesh was used. This division was made to reduce the number of elements and guarantee a sufficiently refined mesh near to the blades, even that the turbulence model uses wall functions for the treatment near to the wall.

For mesh validation, the power and thrust coefficients were used since they are the studied parameters even that additional refinement is necessary to achieve a more accurate pressure distribution in the blades. However, for performance computation purposes, a coarser mesh in the blade region with a wall treatment is sufficient regarding the computational time and hardware limitations.

In the Attachment $\mathrm{C}$, the wall functions are detailed and the ANSYS meshing recommendations are reviewed. For reasonable results, using the wall functions, it is needed a minimum of 10 elements inside the boundary layer, which is achieved even for the blade pitch, with highest flow velocities. 
Figure 33 - Mesh details in turbine blade region

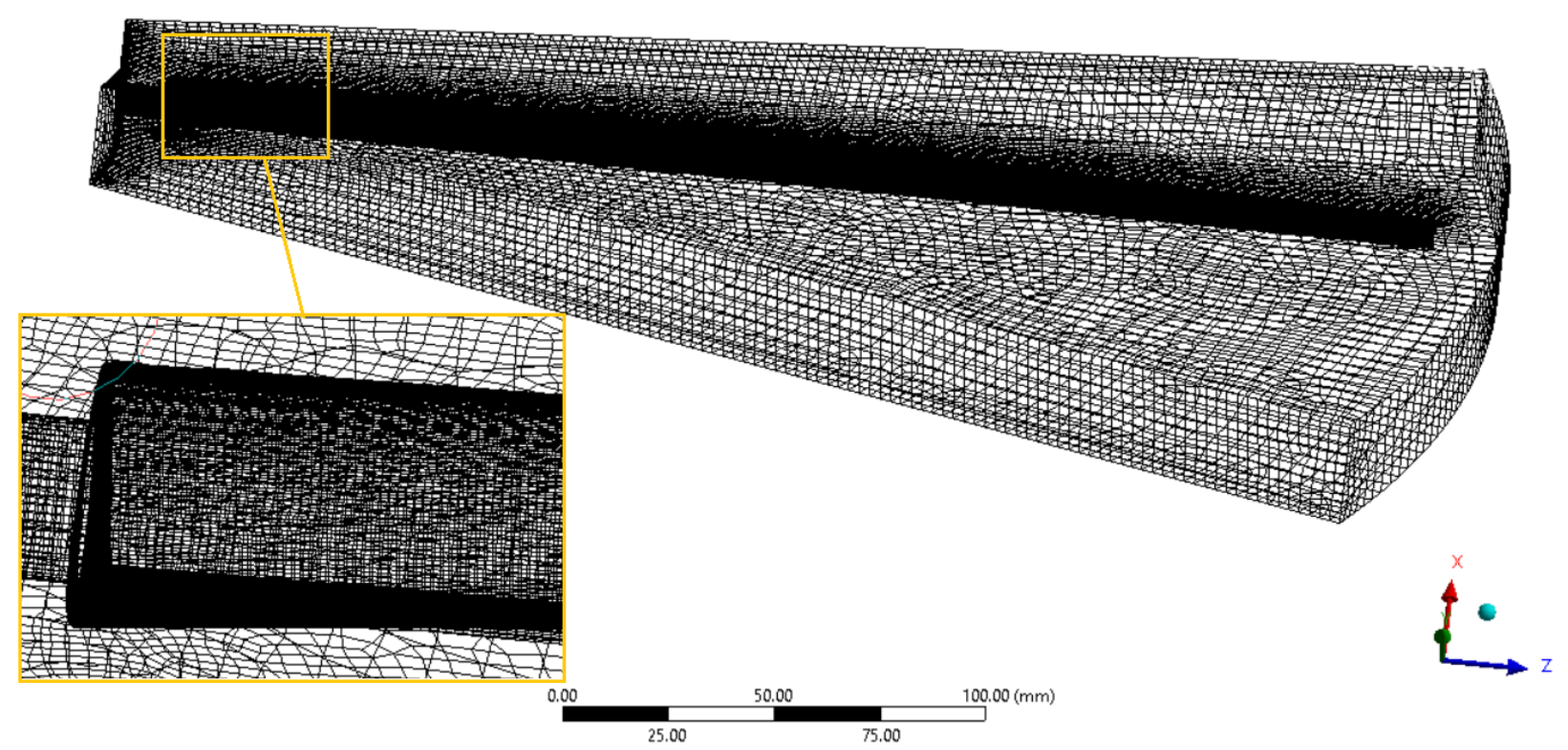

In all the simulations, an Intel® i7 $4510 \mathrm{U}$ processor with 2 cores and 4 threads was used but with the parallel processing limited to 3 threads. This processor has a nominal clock of $2.00 \mathrm{GHz}$ and can be boosted to $3.1 \mathrm{GHz}$. The RAM memory is limited to $16 \mathrm{~GB}$, but since that all other activities consume part of the RAM, the solver uses about $9 \mathrm{~GB}$ for solving the CFD problem in the chosen mesh. It is possible to uses an additional memory from the HD. However, this process slows down the processing time.

The Table 6 shows the summary of the simulations performed for the mesh validation. The convergence was considered achieved when pressure and velocity residuals were all above $10^{-5}$. The $y^{+}$was considered as the mean value in the blade. 
Table 6 - CFD Mesh Validation - Open Flow - TSR $=6, \operatorname{Re}=1.3 \times 10^{6}$

\begin{tabular}{|c|c|c|c|c|c|c|}
\hline $\begin{array}{c}\text { Blade Wall } \\
\text { Sizing [mm] }\end{array}$ & $\begin{array}{c}\text { Domain } \\
\text { Sizing } \\
{[\mathrm{mm}]}\end{array}$ & $\begin{array}{c}\text { Power } \\
\text { Coefficient } \\
{[-]}\end{array}$ & $\begin{array}{c}\text { Thrust } \\
\text { Coefficient }[-]\end{array}$ & $\begin{array}{c}\mathbf{y}^{+} \\
{[-]}\end{array}$ & $\begin{array}{c}\text { Comp. } \\
\text { Time [h] }\end{array}$ & $\begin{array}{c}\text { Number of } \\
\text { Elements } \\
{[-]}\end{array}$ \\
\hline 0.50 & 20 & 0.321 & 0.852 & 149 & 13 & $5.2 \times 10^{6}$ \\
\hline 0.50 & 10 & 0.339 & 0.861 & 152 & 15 & $5.5 \times 10^{6}$ \\
\hline 0.25 & 6 & 0.405 & 0.883 & 73 & 52 & $9.2 \times 10^{6}$ \\
\hline 0.40 & 6 & 0.391 & 0.874 & 121 & 23 & $7.2 \times 10^{6}$ \\
\hline 0.50 & 6 & 0.345 & 0.871 & 156 & 16 & $5.7 \times 10^{6}$ \\
\hline
\end{tabular}

Figure $34-\boldsymbol{y}^{+}$used in the turbine $-\mathrm{TSR}=6.0$

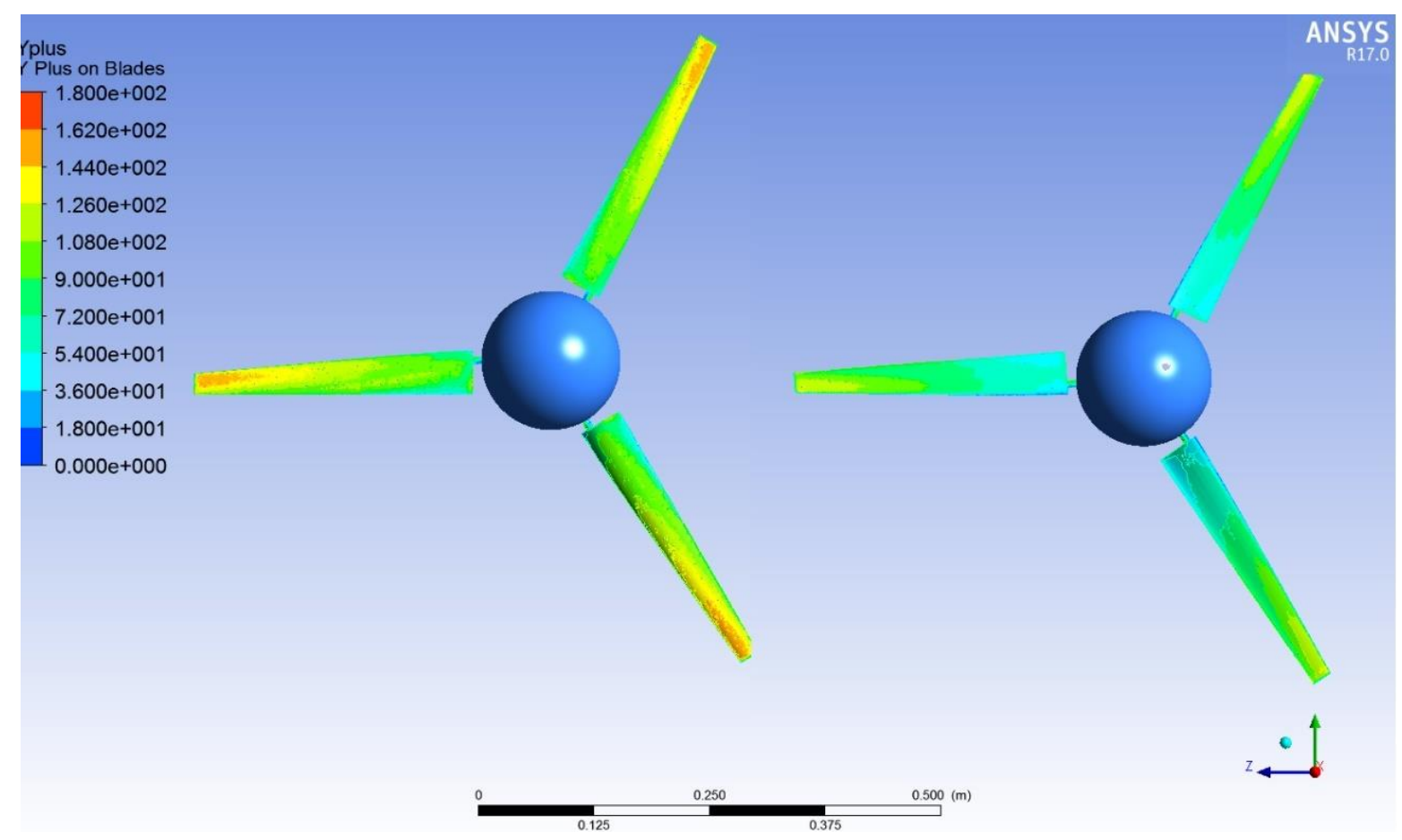




\subsubsection{Subdomains and Domain Interfaces}

The domain has been divided into subdomains to simplify meshing process and analyzing the rotational effects of the turbine. The subdomains are:

- Rotating Domain: Includes the turbine blades and hub. In this domain, it is applied rotation, therefore are applied a source term for Centrifugal and Coriolis body forces and the walls included in this domain, in this case, blades and hub, becomes movable according to a rigid body motion law.

- Undeformable Static Domain: Include the fluid domain excluding the rotational parts for the open domain and confined domain, and excluding the region close to free surface. The mesh of this domain does not present deformation.

- Deformable Static Domain: Includes the region close to the free surface for open channel analysis. The mesh from this domain is deformable.

The Figure 35 illustrates the rotating domain. The interfaces between the rotating domain and static domain are defined as a general connection, with a Frozen Rotor as frame change model, without offset. The program respects the conservation equations of all variables and the mesh connection is defined as General Grid Interface (GGI). All other interfaces between subdomains and parts from subdomains, even in the rotational domain and deformable static domain, are defined as general connection without frame change model.

Figure 35 - Rotating Domain used in all simulations

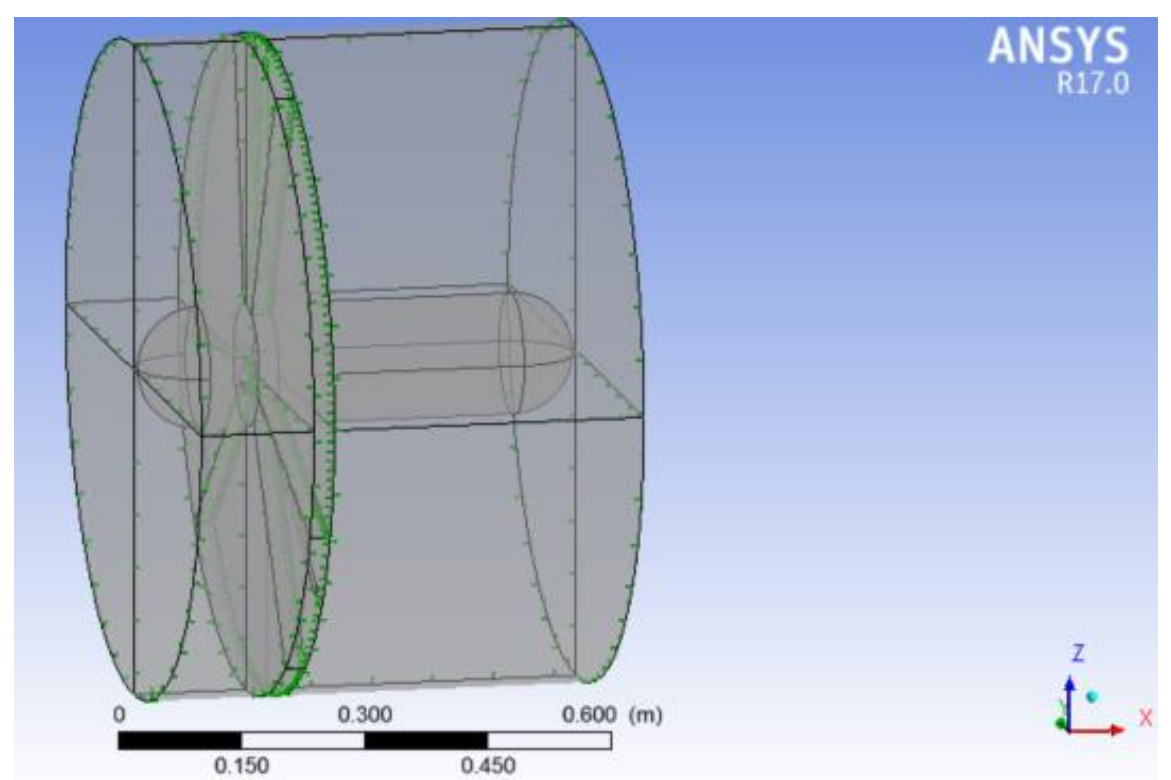




\section{General Boundary Conditions}

For taken into account the effects for considering a non-inertial referential, sources are added to the rotating domain. This source is calculated by:

$$
S_{M, r o t}=-\rho\left[2\left(\Omega_{j} U_{k}\right) \epsilon_{j k i}+\left(\Omega_{j} \cdot r_{j}\right) \Omega_{i}-\left(\Omega_{j} . \Omega_{j}\right) r_{i}\right]
$$

The term $2\left(\Omega_{j} U_{k}\right) \epsilon_{j k i}$ corresponds to the Coriolli's acceleration and the $\left(\Omega_{j} \cdot r_{j}\right) \Omega_{i}-$ $\left(\Omega_{j} . \Omega_{j}\right) r_{i}$ corresponds to the centrifugal acceleration.

The walls, shown in Figure 36, rotates along the turbine axis. The boundary condition implemented in these regions is that the water velocity is equal to the surface velocity since the blades and hub are defined as no-slip walls.

The inlet is a velocity inlet with $5 \%$ of turbulence intensity. This value is the standard of Ansys CFX $\AA$, and according to (Blackmore et al., 2014) the turbulence inlet intensity affects the wake profile and recovery substantially but has a small influence in the turbine power and thrust. The outlet is defined as a pressure outlet, with a pressure specified as zero for the open domain and confined domain and determined iteratively for the open channel domain since the inlet pressure must be equal to the solid pre-stress. While the other domain boundaries are defined as slip-free walls to not produce a head loss and do not affect the inlet and outlet velocity profiles since this analysis is not performed in this work.

The upstream and downstream lengths defined for this work are respectively $10 \mathrm{D}$ and $40 \mathrm{D}(8000 \mathrm{~mm}$ and $32000 \mathrm{~mm})$, values used on references.

Figure 36 - Rotating Walls

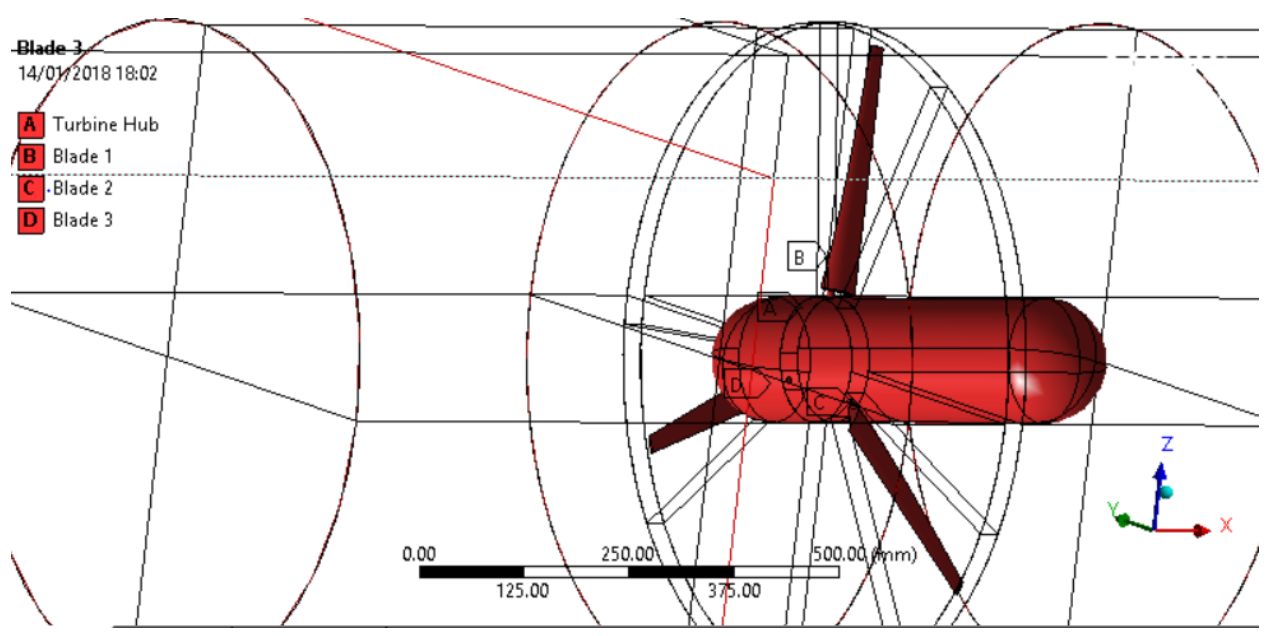




\subsection{Open Domain}

The open domain is schematized on the Figure 37. The static domain is constituted by blocks to provide a higher refinement in the wake region maintaining the mesh regularity.

The inlet boundary condition consists in a constant velocity condition with the axial component with $1.73 \mathrm{~m} / \mathrm{s}$ and $5 \%$ of turbulence intensity (default from Ansys). The outlet was defined as a pressure outlet with $0 \mathrm{~Pa}$ of relative pressure. In the external diameter of the domain, the pressure is defined as $0 \mathrm{~Pa}$, which is reasonable regarding to the conditions used to calculate the Betz Limit. The pressure drop is negligible if the pressure drop energy is compared with the total amount of kinetic energy available in the inlet flow.

The domain dimensions were adjusted to make the results independent of the domain size. For upstream length $\left(L_{u}\right), 10 \mathrm{D}$ was used, totalizing $8 \mathrm{~m}$ and for downstream length $\left(L_{D}\right), 40 \mathrm{D}$ was used, totalizing $32 \mathrm{~m}$. The total length of the domain is $40 \mathrm{~m}$. For the refined part of the domain, it was defined $0.5 \mathrm{D}$ in upstream and $1.5 \mathrm{D}$ in downstream, with a mesh sizing of $20 \mathrm{~mm}$. For the whole domain, a mesh size of $100 \mathrm{~mm}$ was used. The mesh convergence test indicates that the results dependency on the mesh sizing on the domain is negligible if compared with the dependency on the turbine disk region.

The external diameter of the domain is equal to $10000 \mathrm{~mm}$ providing a blockage equal to $0.64 \%$ that according to (Garret \& Cummins, 2007) increases the power coefficient in $0.93 \%$, which is negligible if compared with the difference between numerical and experimental results.

Figure 37 - Open Domain Schematic Drawing

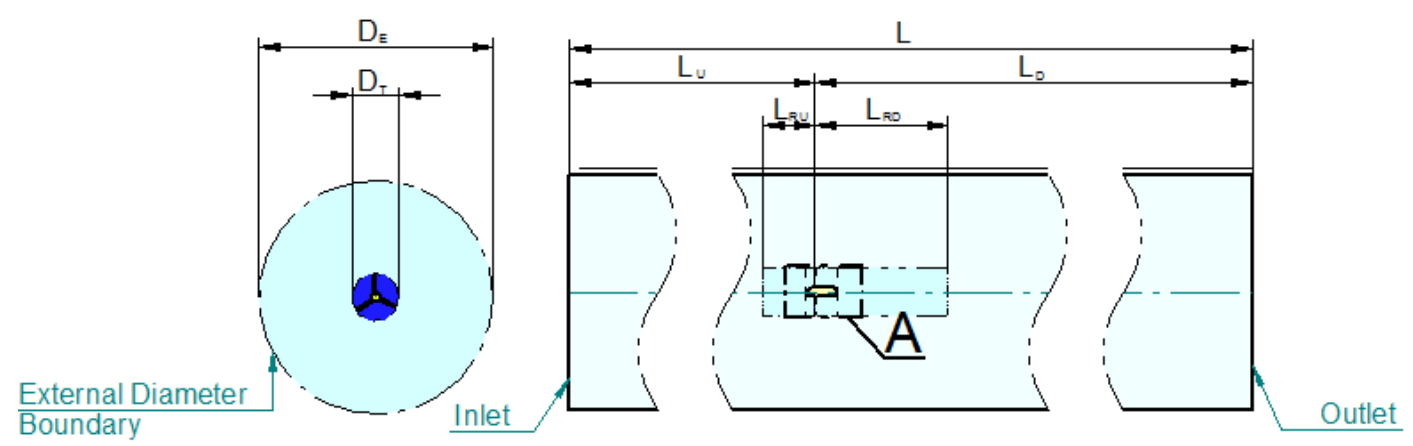




\subsection{Confined Domain}

Both confined domain and open channel domain uses the same 3D model for the analysis. The domain is divided into three regions:

- Rotating Domain

- Static Domain

- Deformable Domain

The deformable domain is defined as rigid for this case since no mesh deformations are required. The inlet boundary condition is the same as used for open domain, with constant velocity with an axial component of $1.73 \mathrm{~m} / \mathrm{s}$ and $5 \%$ of turbulence intensity. The outlet is defined as a constant pressure outlet, with $11700 \mathrm{~Pa}$, according to the Table 2. The program calculates the inlet pressure automatically with these boundary conditions. All walls are modeled as slip-free walls since, in this work, the influence of profiled flow and channel boundary layer are not considered. The domain upstream and downstream lengths are the same as used in the open flow model. The dimensions of the channel are $2.4 \mathrm{~m}$ of width by $1.2 \mathrm{~m}$ of height, providing a blockage factor of $17.4 \%$, in which is expected a power increase of $45 \%$, according to (Garret \& Cummins, 2007).

\subsection{Open Channel Model - Elastic Free Surface}

The two main differences between this model and the confined domain model are concerning the deformable domain and the free surface region. The deformable part of the domain, defined as the upper $100 \mathrm{~mm}$ of height have mesh deformation based on displacement diffusion, in which the mesh is updated about the previous mesh, that for a mesh with all elements with the same size becomes (ANSYS Inc., 2016, p. Section 1.1.2)

$$
\nabla^{2} \delta=0
$$

The free surface is coupled with the orthotropic solid by the ANSYS Workbench ${ }^{\circledR}$ Coupling, The pressure field in the free surface is transported to the solid, then the program solves the solid model for obtaining the free surface deformation to update the fluid model again. The solid model height is $3.6 \mathrm{~m}$, providing an elasticity modulus of $35100 \mathrm{~Pa}$ for obtaining a transversal stiffness of $9778.2 \mathrm{~N} / \mathrm{m}^{3}$. The solid is pre-stressed with $11700 \mathrm{~Pa}$. 


\section{Chapter 8. Results}

In this section, are shown the primary results of the three CFD models and the comparison between their results. The open domain flow is used mainly for validation since the experimental data has a blockage correction for guarantee the independence between the results and the boundary conditions. The confined flow and the free surface flow results are presented in order to analyze the influence of the boundary conditions in the turbine hydrodynamic performance.

\subsection{Open Domain}

For open domain flow, it is shown that the turbine has a low influence in the far flow. The velocity distribution, shown in the Figure 38, indicates a high-velocity deficit in the wake region, in a distance below of 2 diameters in the downstream direction. A small velocity increasing is presented in the bypass region. This increase occurs due to the flow change of direction due to the presence of the turbine disk. The streamlines, shown in Figure 39, are not affected in higher domain diameters, presenting no velocity gradients and no change of direction.

The velocity quickly drops in the wake region, and for fulfilling the mass conservation, the cross-section in this region has a higher diameter than the turbine disk, as predicted by the actuator disk theory.

Figure 38 - Velocities on Symmetry Plane for TSR $=6$

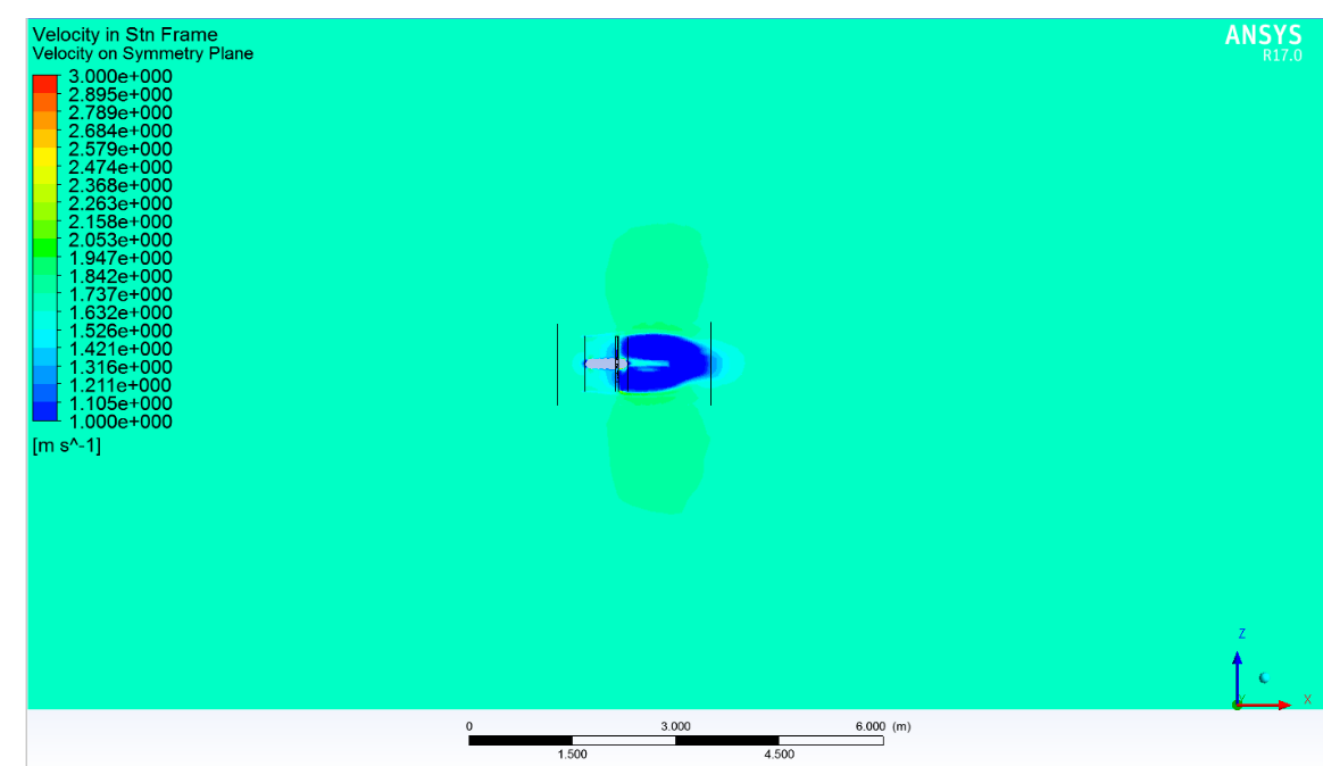


Figure 39 - Typical streamlines for open domain flow

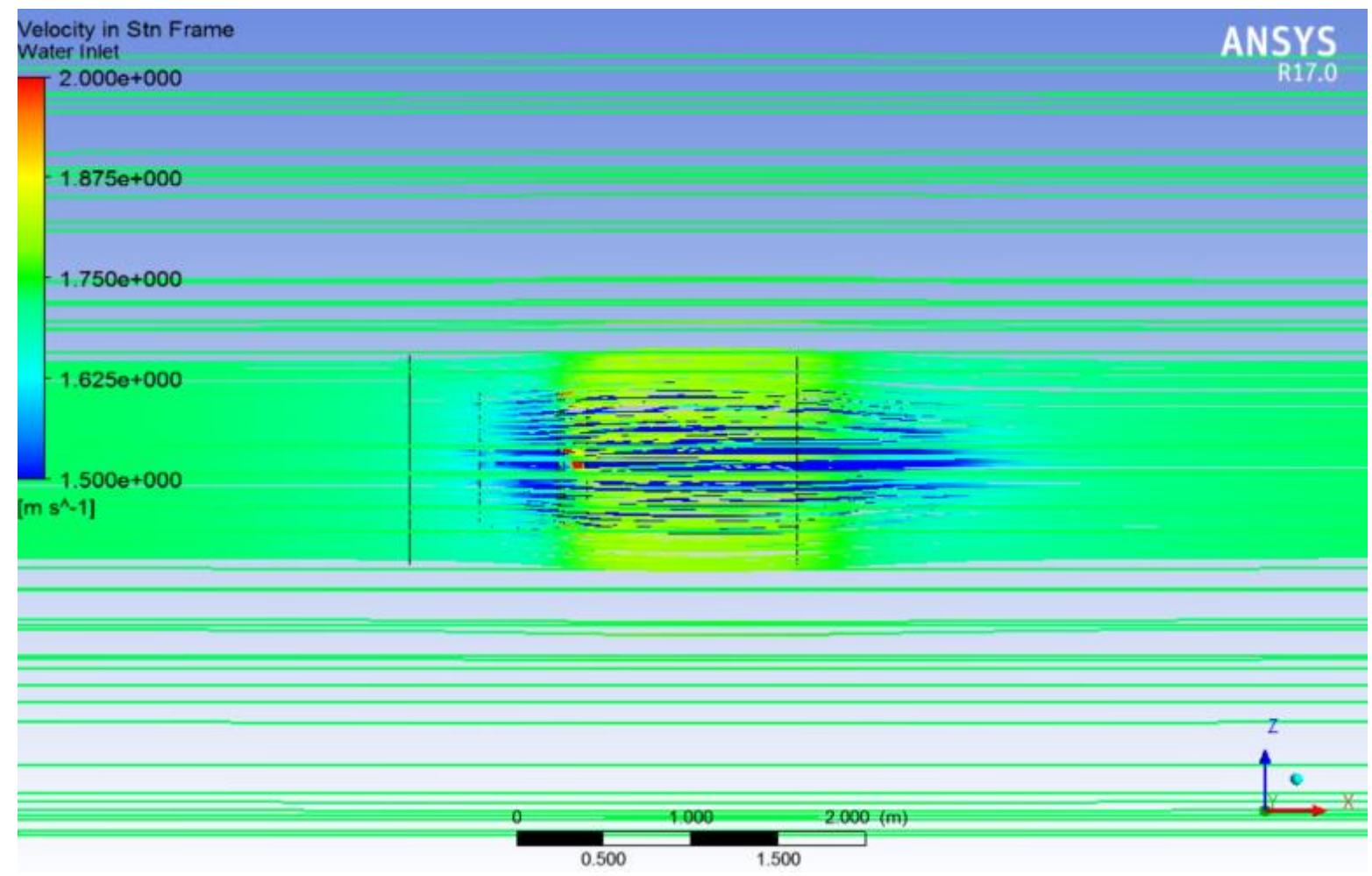

In Figure 40 the streamlines through the turbine disk are compared for different TSR's. For the lower TSR, it is clearly shown the turbine has low influence in the outer flow. The velocity quickly recovers in the wake and the inlet and outlet areas are almost the same. For higher TSR values, the wake is presented a velocity deficit. Near to the peak $\boldsymbol{C}_{\boldsymbol{P}}$, found for TSR $=5.625$, the wake has a higher length if compared with TSR $=8$. More power is taken from the flow requiring a higher length for the recovery. The higher TSR presents a shorter wake length with the highest section area, which is expected since for a higher rotation is expected that the turbine affects the flow in the same way as a disk. 
Figure 40 - Streamlines through turbine disk for Open Flow

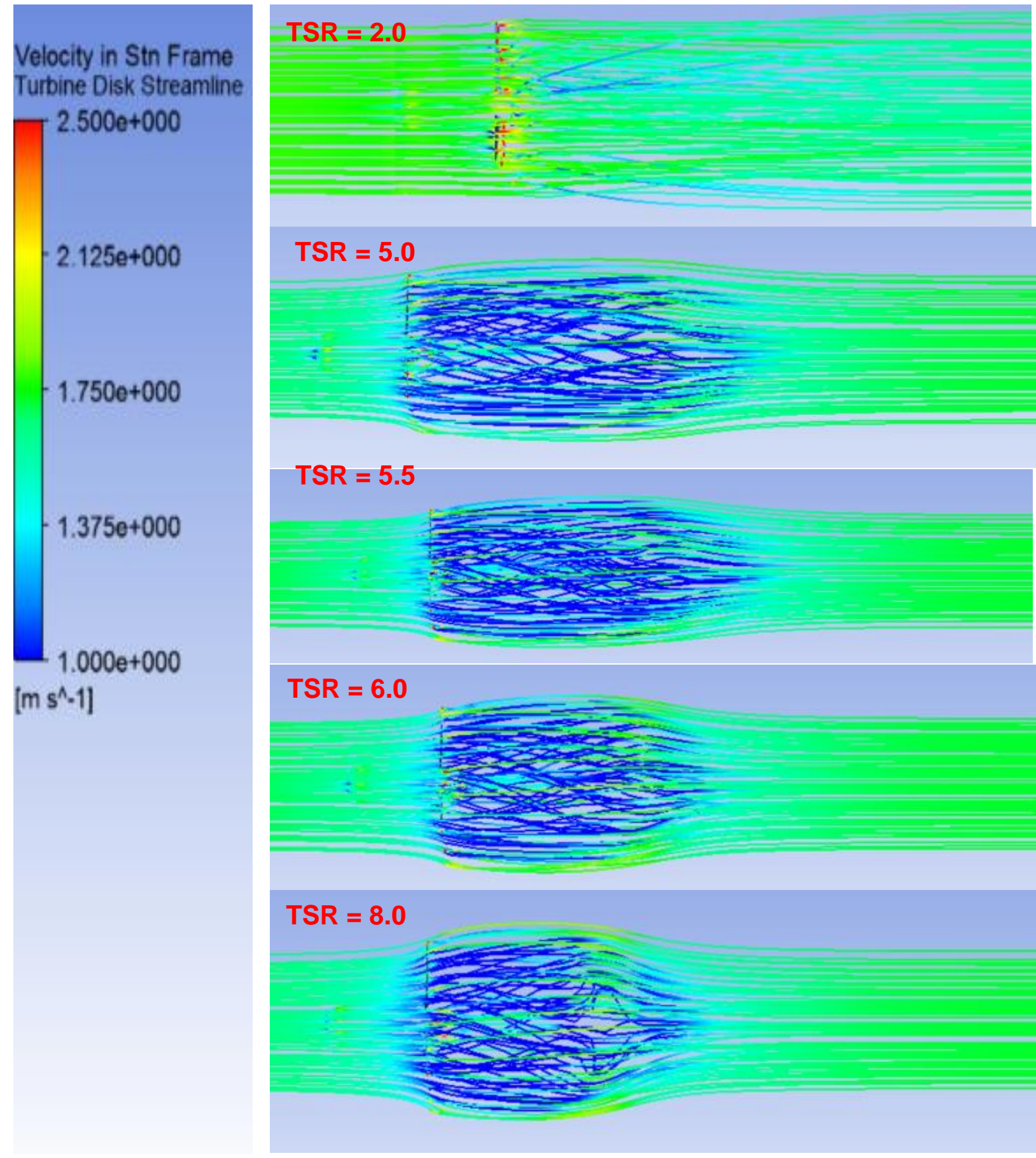


The comparison between the figures above shows the difference between the pressure distributions in the blades for different TSR's. It is seen that for the TSR = 6.0 , the pressure on the suction side is lower than the calculated for TSR $=5$ and $\mathrm{TSR}=5.5$. It occurs due to the higher absolute speed of each blade section. Even with lower effective attack angles, this increase in the speed leads to higher pressure drops.

Figure 41 - Pressure on Blades for TSR $=5.0$

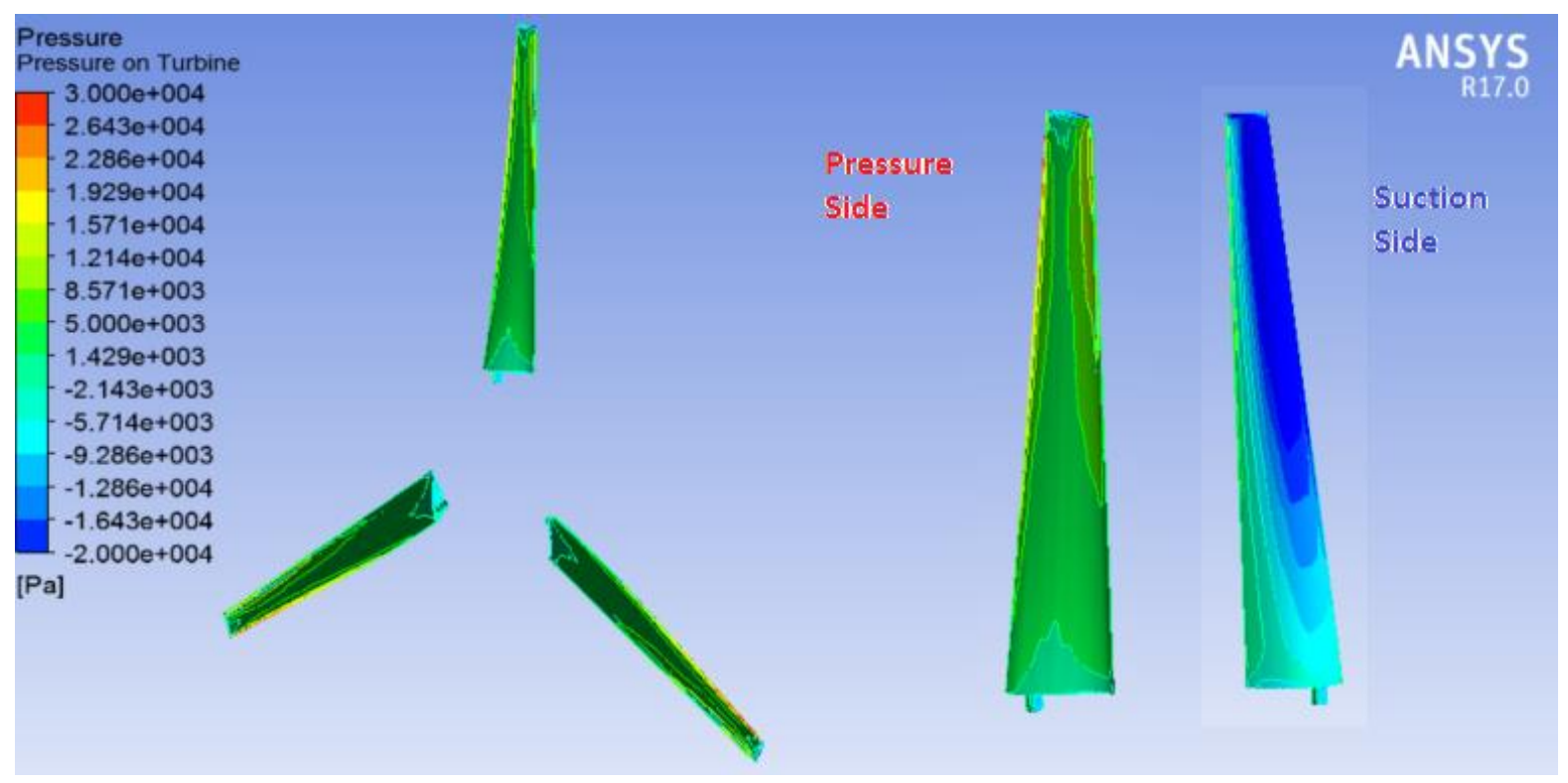

Figure 42 - Pressure on Blades for TSR $=5.5$

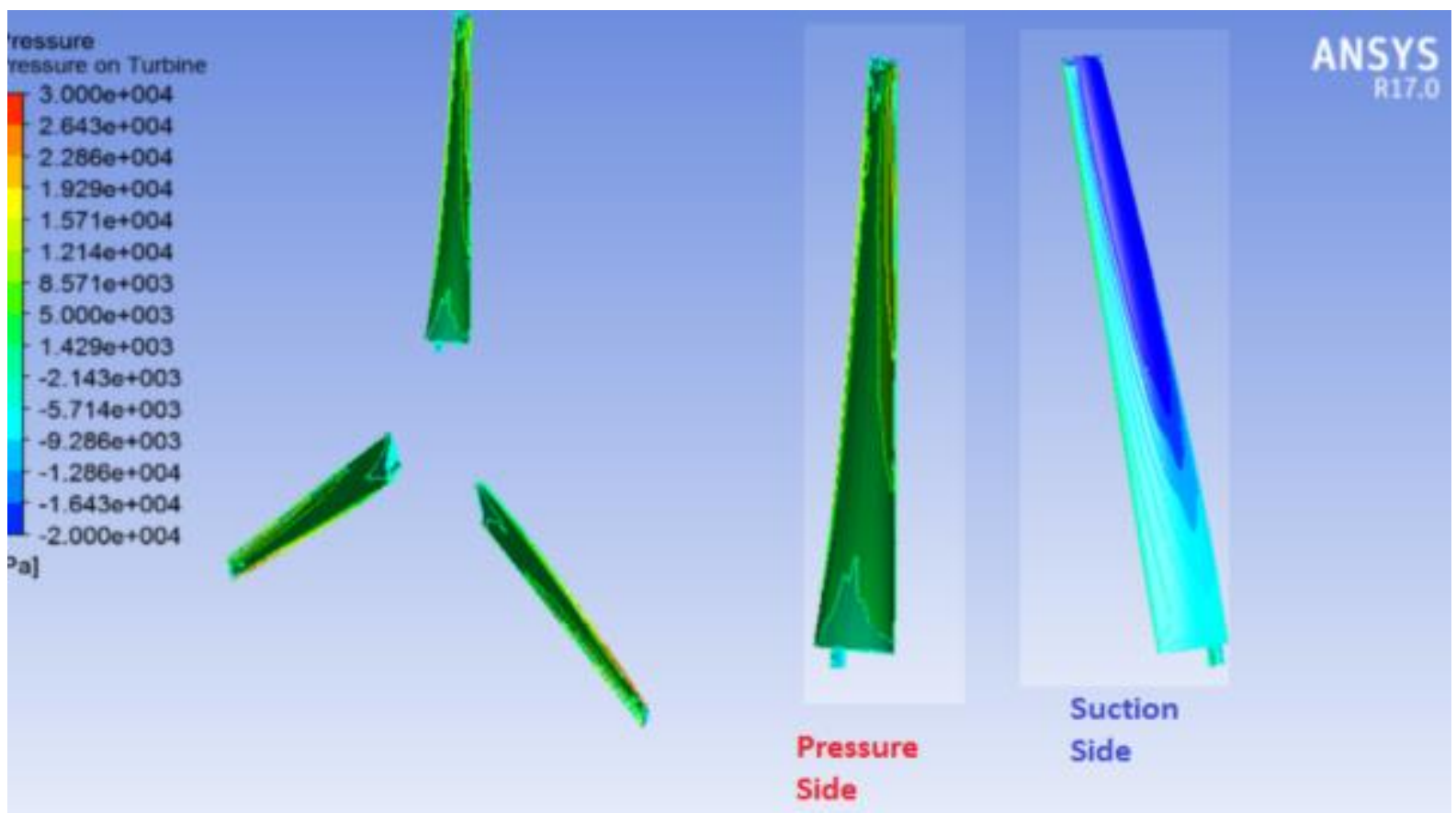


Figure 43 - Pressure on Blades for TSR $=6.0$

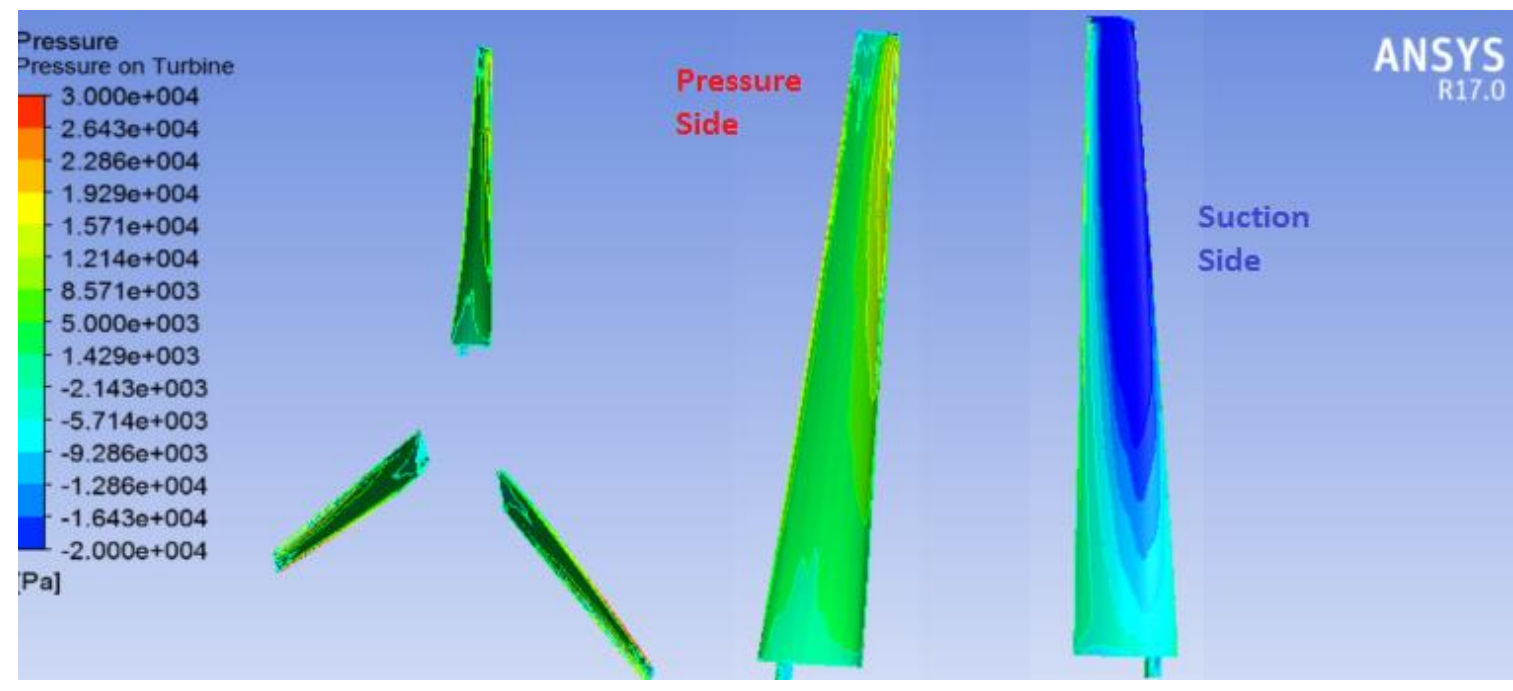

The expression for attack angle, according to the Blade Element Momentum Theory for each blade section is shown above:

$$
\alpha_{T}(r)=\operatorname{atan}\left(\frac{1}{T S R} \cdot \frac{R}{r} \cdot \frac{1-a}{1+a^{\prime}}\right)-\beta_{p}
$$

Where:

a Is the axial induction factor which decreases the axial velocity;

$a^{\prime} \quad$ Is the tangential induction factor which increases the tangential velocity.

Is seen that for higher TSR's, the attack angle of each blade section decreases, which decreases the effective attack angle. It is known that stall is associated with high attack angles, which happens mainly due to the flow separation in the blades, decreasing the lift and hence the hydrodynamic performance of the blade.

The Figure 44 shows the wall shear distribution for the pressure and suction sides of the turbine blade for three different TSR's. When the absolute value of wall shear is equal to zero, it is indicated that the velocity gradient is equal to zero and hence flow recirculation may occur. Therefore, zero wall shear indicates the flow separation phenomena. For TSR $=5$, flow separation occurs in almost all blade near to the trailing edge, which is not seen for TSR $=6$. These results match the theoretical predictions according to the BEMT methodology. The increase of angular speed of turbine blade decreases the attack angle. However, near to the stall angle, it is found the maximum lift force which produces a torque which is increased with hydrodynamic attack angle. 
Figure 44 - Shear stress on blades

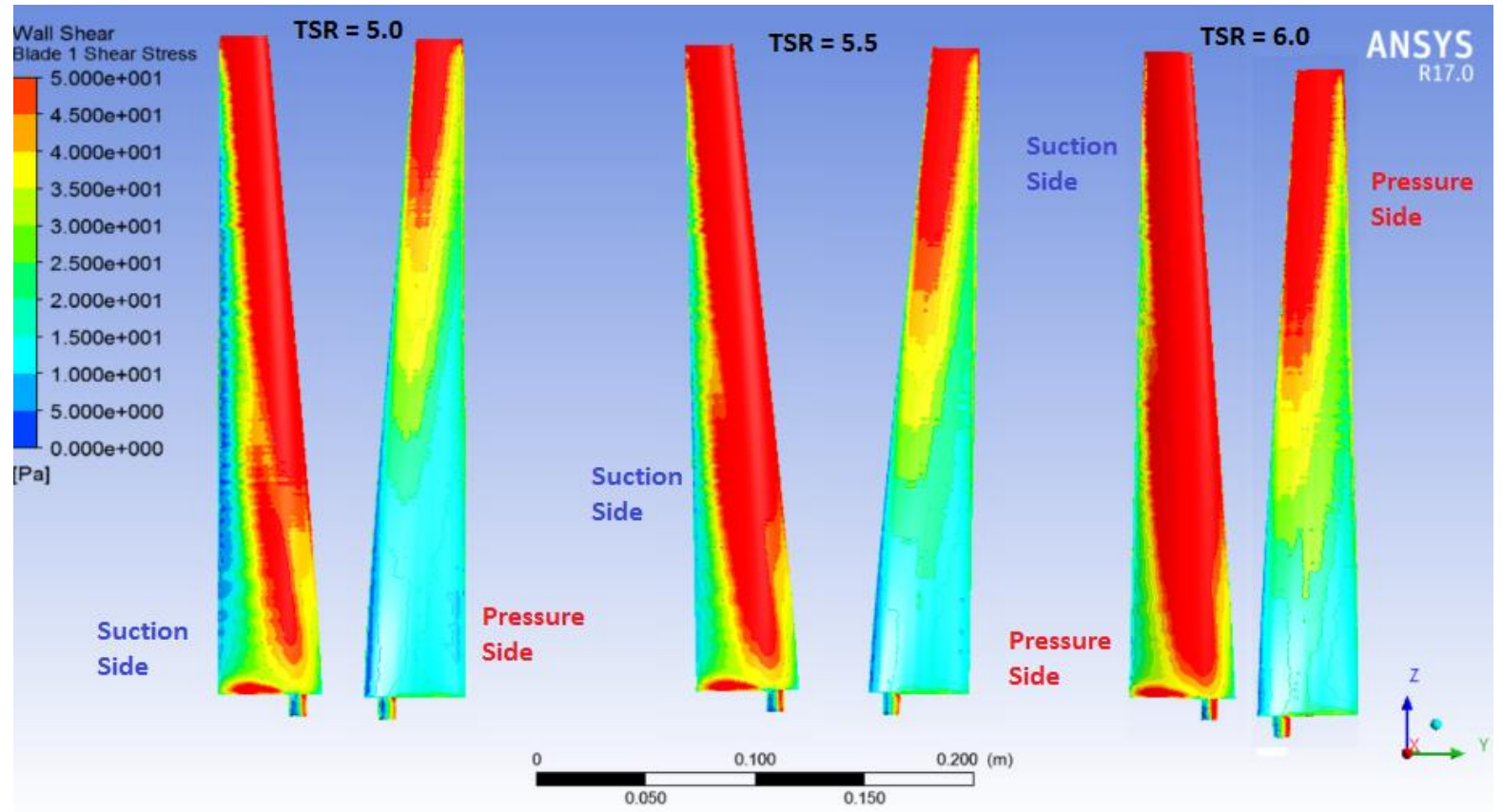

The figures above show the comparison between the experimental results and the CFD results. The power coefficient is underpredicted if compared with the experiments and the thrust coefficient is overpredicted. The increase of $\boldsymbol{C}_{\boldsymbol{T}}$ with the turbine rotation is in which the turbine is closer than a disk, which causes a higher restriction to the flow than a turbine with a lower rotation.

In (Afgan et al., 2012), in which both turbulence models were compared, the CFD results were closer to the experimental analysis for LES, however, analyzing the performance through the same model used in this work, the underprediction is almost the same.

Accordingly, both power and thrust evaluations showed the same trend by experimental methodology and by CFD approach. Therefore, the CFD model used in this work is validated for a first analysis of the tidal current turbine, being useful for a more accurate design of the blade if compared with BEMT. However, for the final geometry, a more robust CFD approach and an experimental analysis still required. 
Figure 45 - Comparison between Experimental and CFD Results: Thrust Coefficient

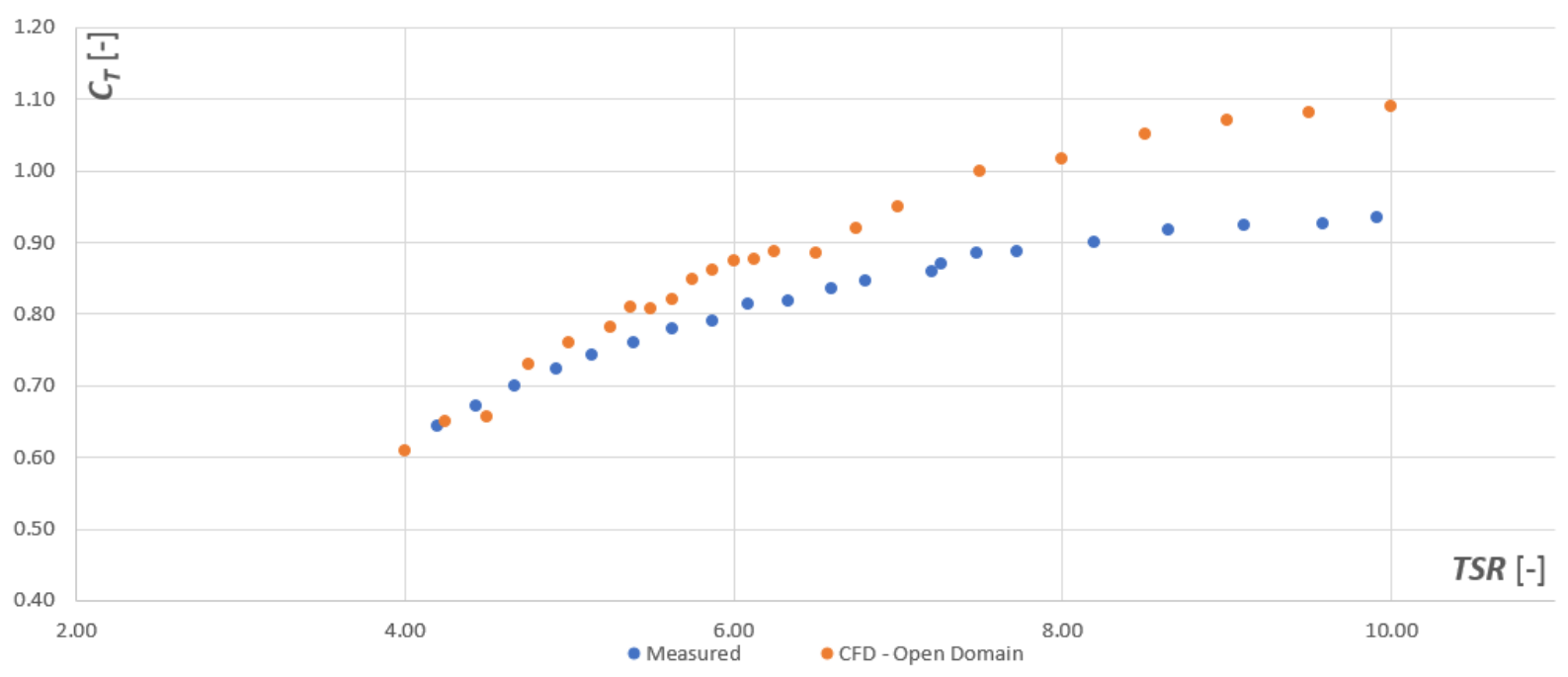

Figure 46 - Comparison between Experimental and CFD Results: Power Coefficient

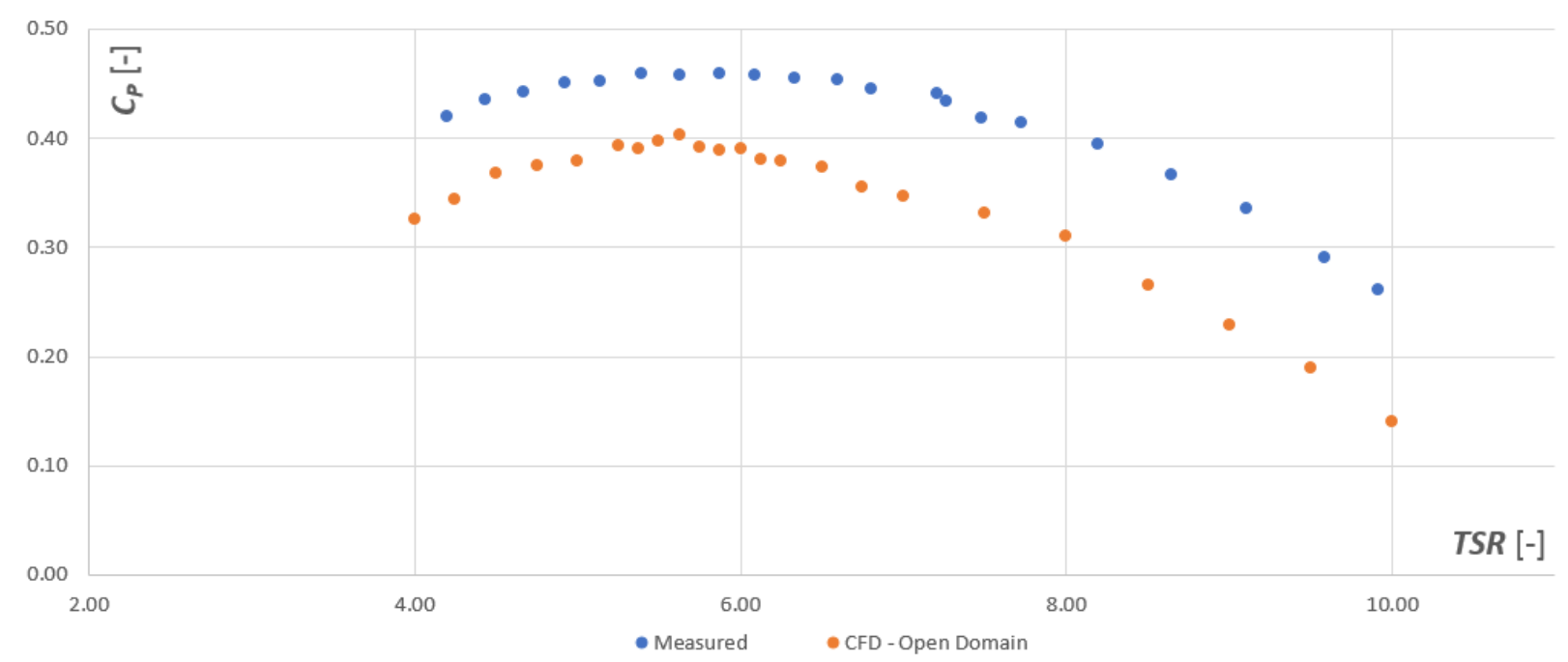




\subsection{Confined Flow}

The Figure 47 shows the streamlines through the axial turbine and the velocity distribution in the symmetry plane for TSR $=6.0$. It is clearly seen the two different flow regions: The flow through the turbine in the bypass region. The velocity in the by-pass is higher than the mean inlet velocity, and this velocity difference is more pronounced than in the open domain analysis, which is considered in the actuator disk theory.

The mixing between the two regions happens in a distance of approximately $5 \mathrm{D}$, and before the mixing, a region with vortex occurs, that happens due to the difference of velocities between the two flow regions, producing a high level of shear stress, and therefore, makes the fluid elements rotate. The Figure 48 shows the velocity curl that is coincident with the interface with the flow that passes through turbine and by-pass.

Figure 47 - Velocity distribution near to turbine - Confined Flow, TSR $=6.0$

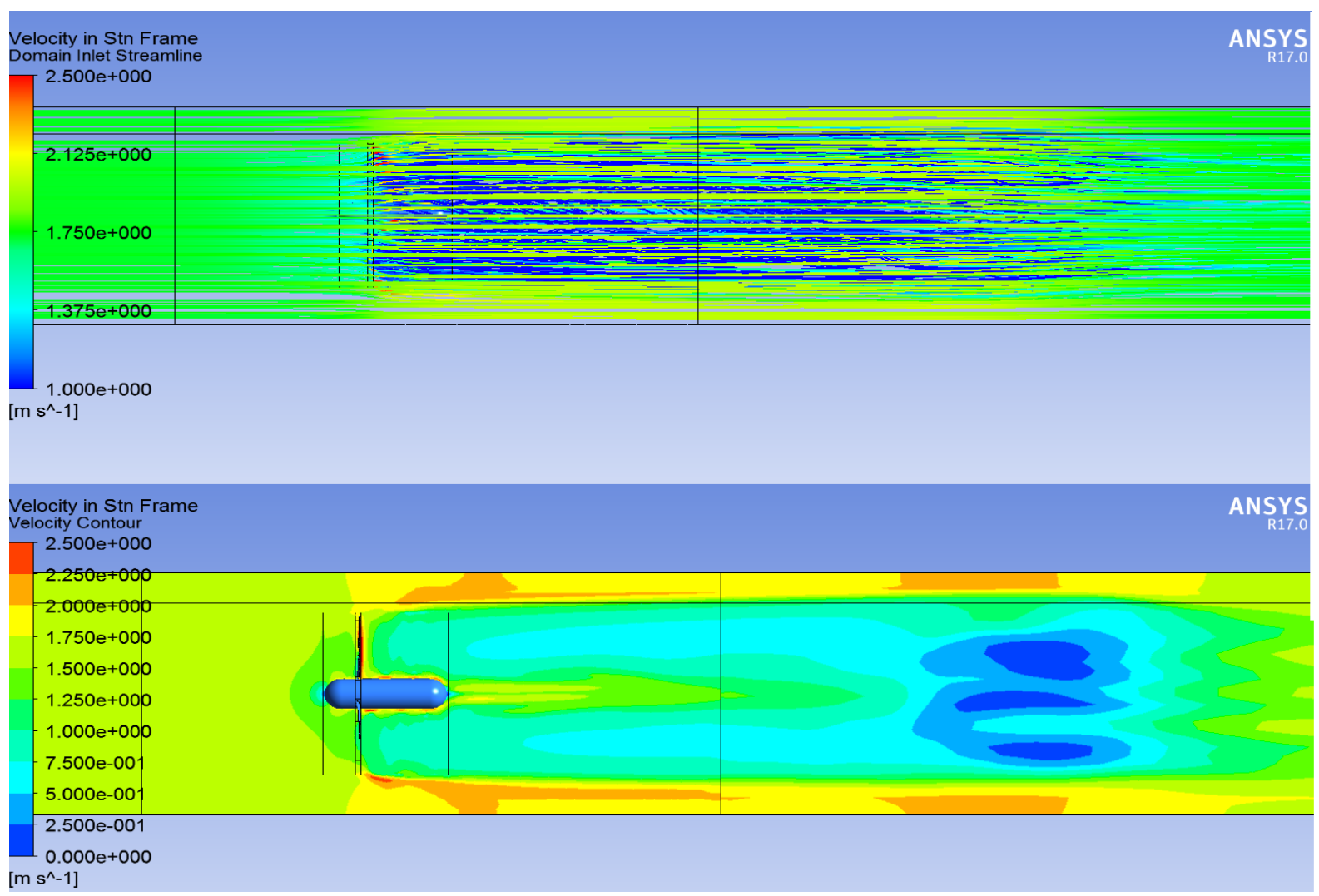


Figure 48 - Velocity Curl for Confined Flow - TSR $=6$

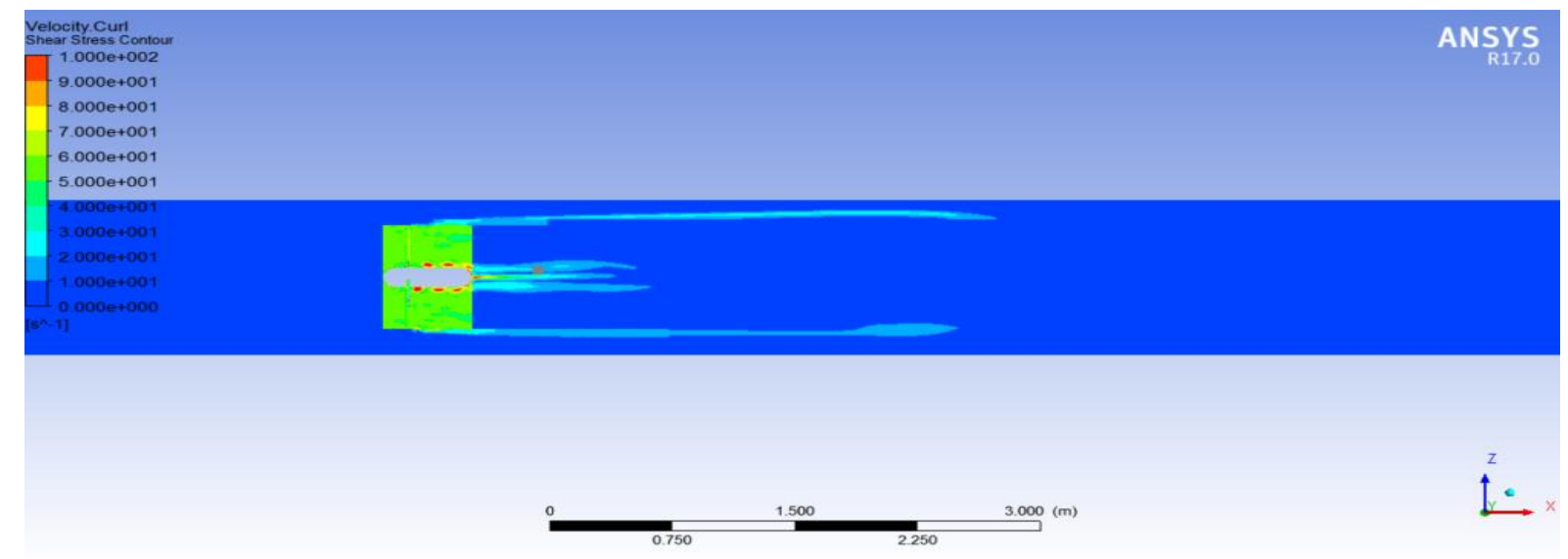

In Figure 49, the differences in the wake between confined domain and open domain are analyzed. The first difference is concerning the wake length. The confined domain has a wake length at least 2.5 times higher than the analyzed for the open domain. The flow confinement transports the vorticity of the wake flow becoming more difficult the dissipation of the flow rotation. This effect is expected since higher turbulence levels decrease the wake length. For confined flows, the by-pass flow has lower inertia if compared with open domain by-pass, which makes the wake more stable. A result of this analysis is that for channel flows with high blockage ratio, even the individual performance of each turbine is expected to increase, and the turbines must be placed within a greater axial distance that could result in a decrease of the total power generated in the farm.

The wake width is lower for the confined domain. It is expected since the confinement restricts the wake expansion. 
Figure 49 - Comparison between wakes for confined flow and open flow - TSR $=6.0$

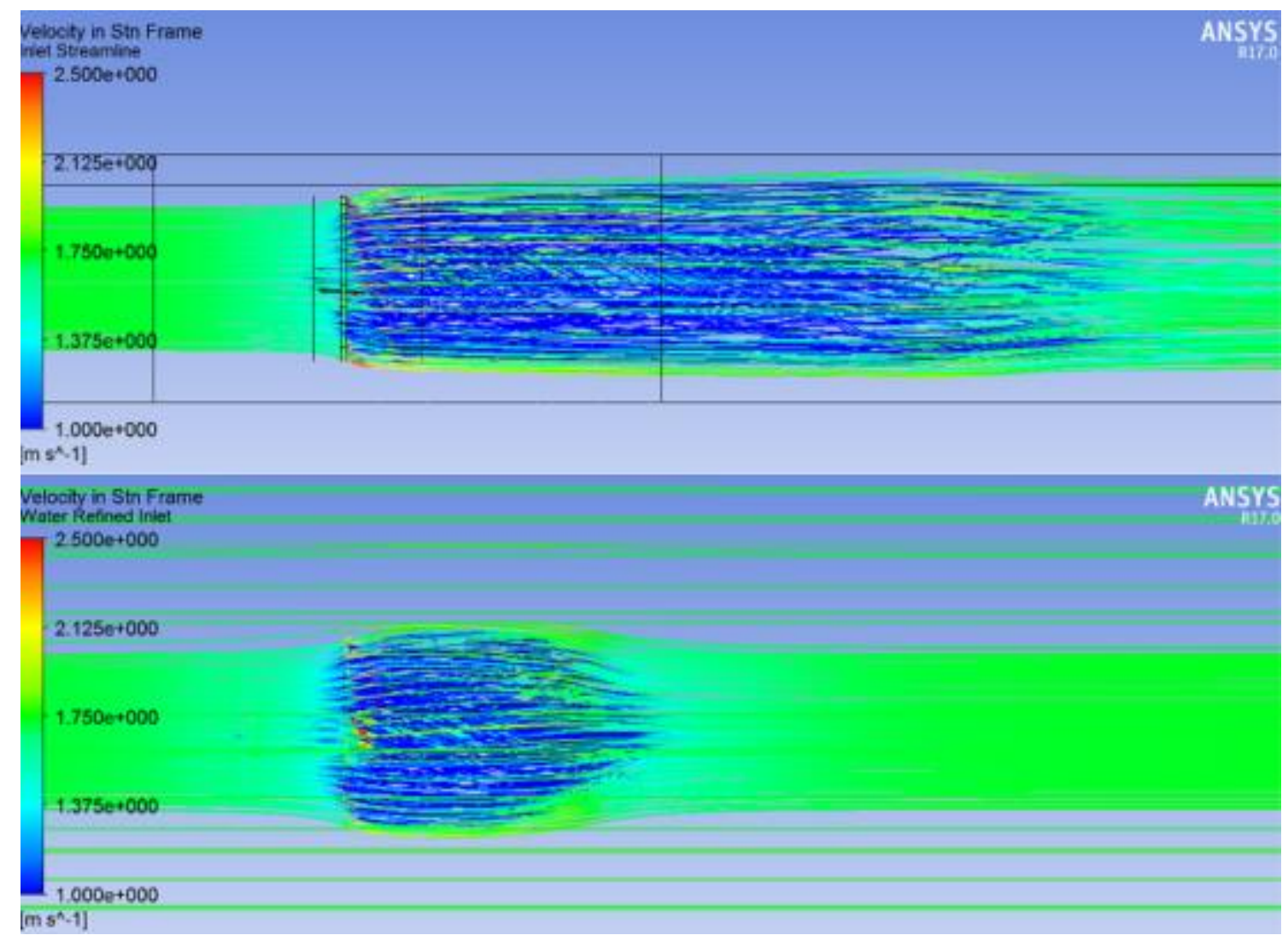

The Figure 50 presents the pressure distribution for the turbine blades. The pressure difference reaches $70 \mathrm{kPa}$ for blade external diameter. The comparison with the results for open flow reveals a higher pressure on the pressure side of the blade even the pressure distribution for the suction side is not affected. The Figure 51 shows the comparison between the wall shear stress, which presents almost the same distribution of shear between the two analyses, revealing the low influence of the flow confinement in the flow separation. 
Figure 50 - Pressure distribution on blades for confined flow

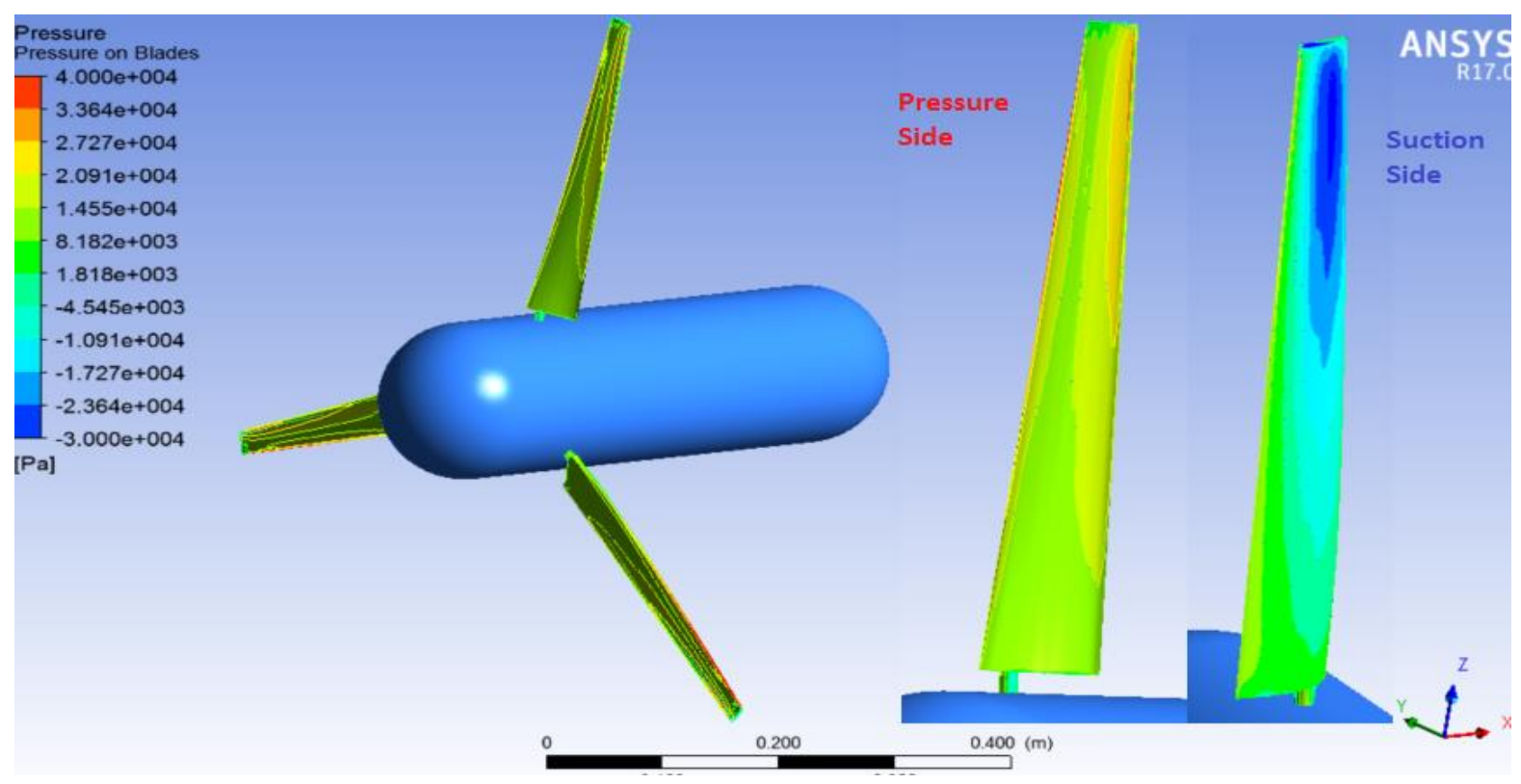

Figure 51 - Comparison between confined domain and open domain - Wall Shear Stress

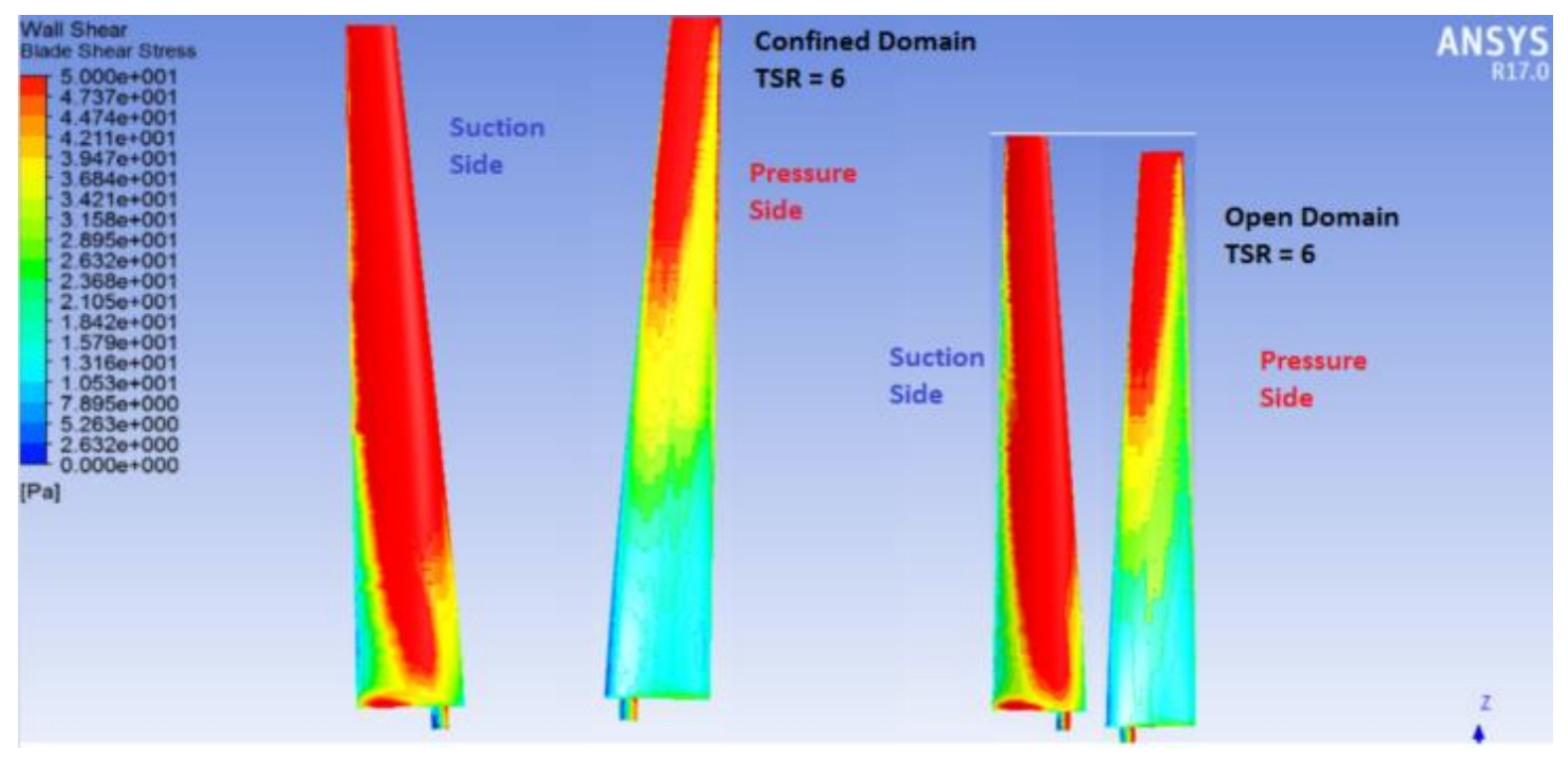




\subsection{Open Channel}

The open channel flow is modeled with an elastic wall approach. However, for the velocity of $1.73 \mathrm{~m} / \mathrm{s}$, the pressure difference between upstream and downstream regions together with the blockage ratio in order of $20 \%$ led to a free surface instability. The streamlines for the model for TSR $=6.0$ are shown in the Figure 53 , it is seen the interference between the turbine wake and the free surface, in which regions with high speed occurs. There flow vorticity is clear near to the free surface.

This interaction made the model diverges after 7 Fluid-Structure coupling iterations due to negative Jacobian elements formed due to the high deformation of the free surface in the downstream region. This problem did not occur in (Mattavo \& Assi, 2017) in which the distance between the obstacle and the free surface is higher than the obstacle diameter.

For analysis of the dimensionless power and thrust coefficients, the simulation was carried out with an inlet velocity of $10 \%$ than the original model, with the rotation decreasing to maintain the same TSR values. This new model resulted in a Reynolds Number of $1.5 \times 10^{5}$. This adjustment is expected to reduce the pressure drop to $1 \%$ of original value and according to the equation (C.26) and the same decreasing is expected for the head difference between the inlet and outlet.

In (Mason-Jones et al., 2012) the authors concluded that for a $R e>3 \times 10^{5}$ the power and thrust coefficients are a function only of TSR, however, since the analyses were conducted without free surface effects, this value could not be used as a reference. However, considering the preliminary stage of development of the elastic free surface methodology, the analysis with this inlet velocity is valid for testing the model capabilities.

The model with inlet velocity of $1.73 \mathrm{~m} / \mathrm{s}$, shown in Figure 52, presented for a TSR equals to 6.0 a $\boldsymbol{C}_{P}$ of 0.29 and $\boldsymbol{C}_{\boldsymbol{T}}$ of 0.79 while the model with the inlet velocity of $0.173 \mathrm{~m} / \mathrm{s}$ shown in Figure 53, presented for the same TSR value a $\boldsymbol{C}_{P}$ of 0.393 and $C_{T}$ of 0.83 . 
Figure 52 - Results for free surface analysis with inlet velocity of $1.73 \mathrm{~m} / \mathrm{s}$

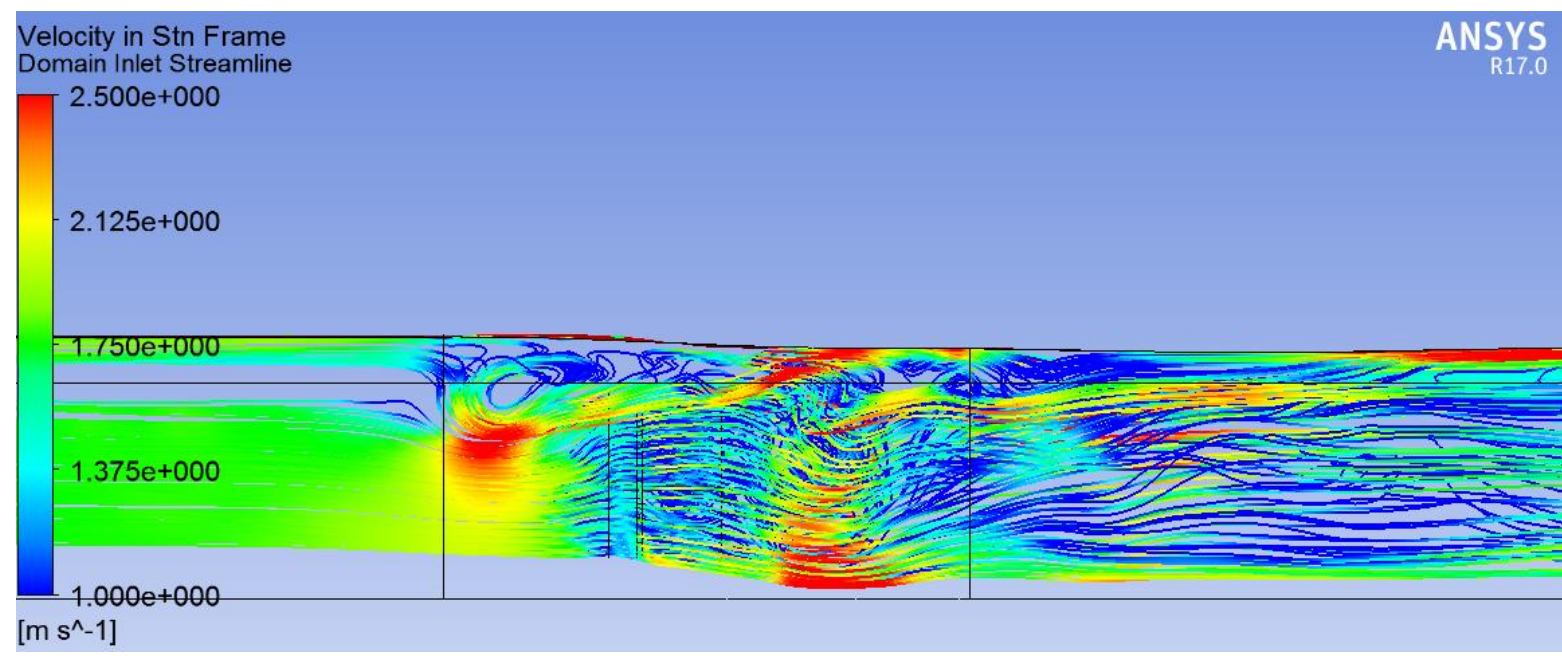

\subsubsection{Flow Analysis}

The comparison between the wake lengths, with the free surface model in Figure 53 and the fully confined and open domain models in the Figure 49, shows that the partial confinement due to the free surface leads to a larger wake than the calculated for the open domain but shorter wake than the calculated for the confined domain. However, in a distance of approximately 8 diameters downstream, the wake started to interact with the free surface.

Figure 53 - Streamlines for open channel flow - TSR $=6$, Inlet Velocity of $0.173 \mathrm{~m} / \mathrm{s}$

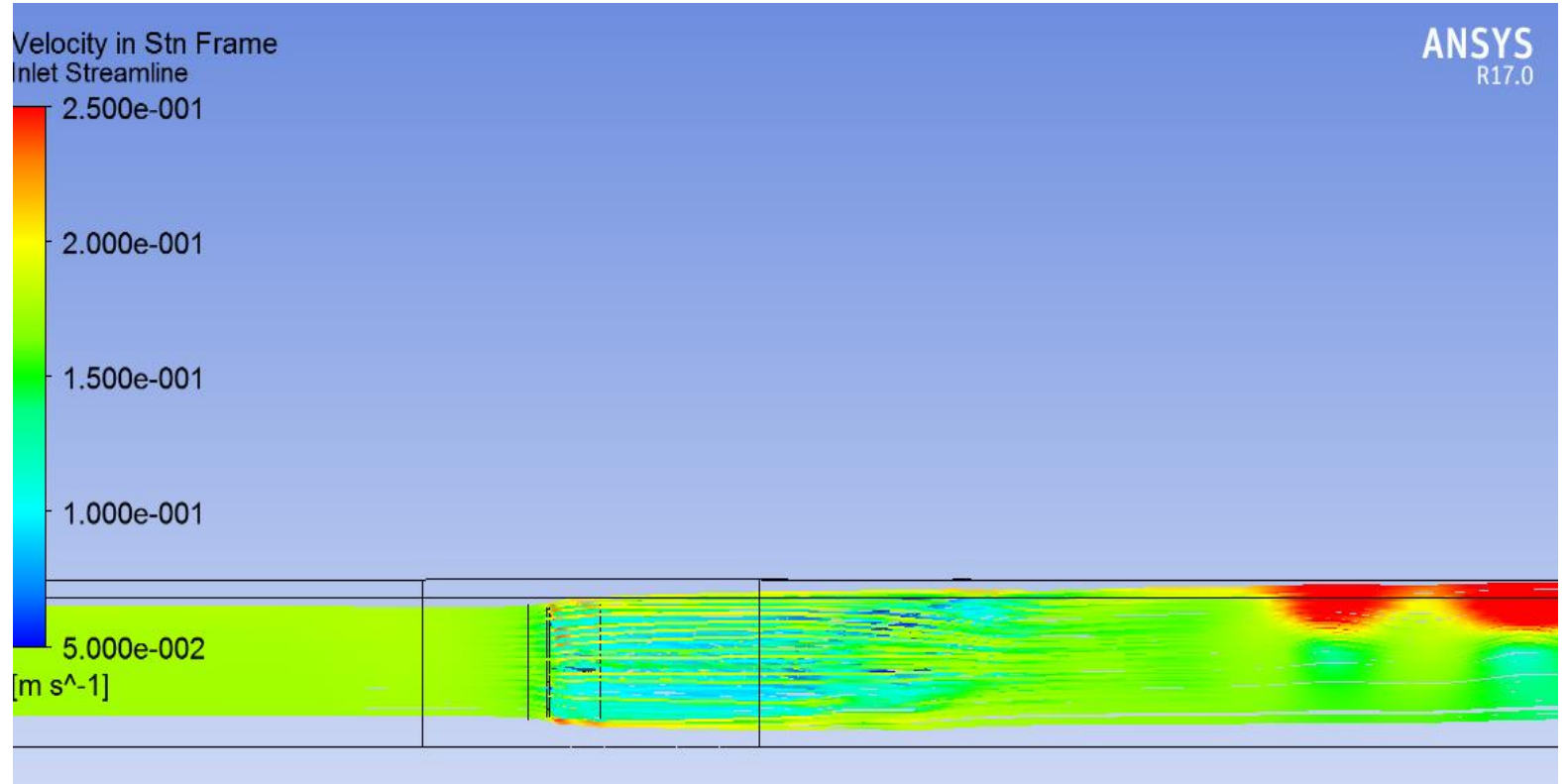


Figure 54 -Detail of Turbine Wake (Left) and Detail of Free Surface Instabilities (Right)
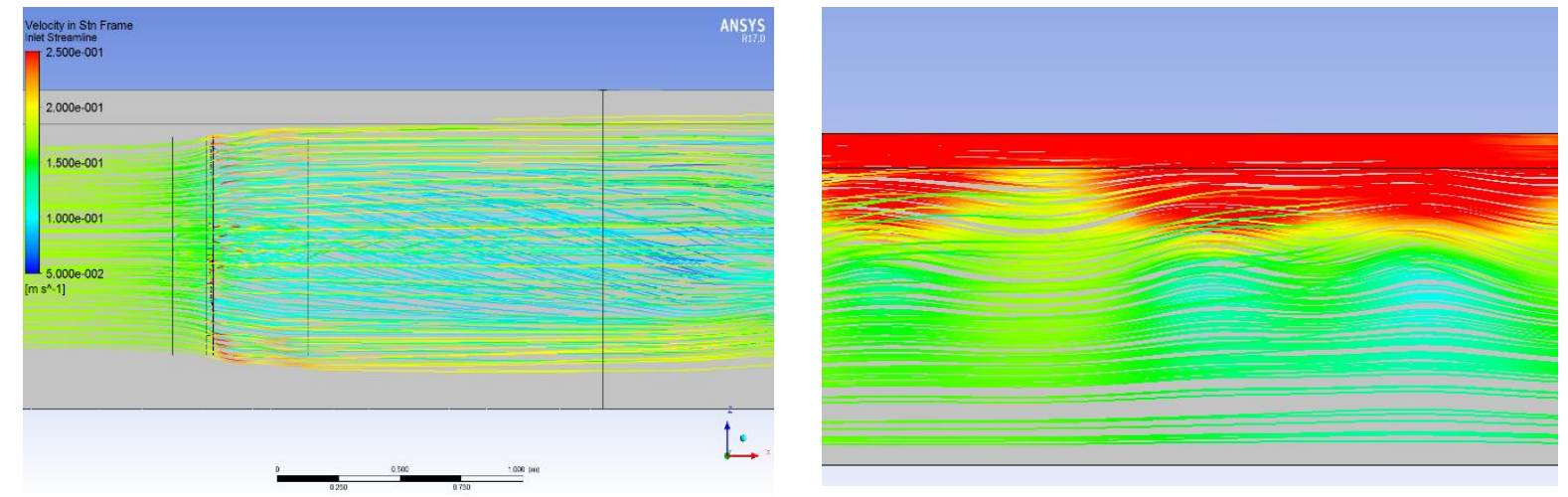

The Figure 55 presents the streamlines for the entire fluid domain. There is an increase in velocity in the turbine by-pass, colored in yellow.

Looking to the downstream part of the domain, far from the free surface, the velocity returns to the inlet velocity, which is expected considering negligible deformations of the free surface. However, in Figure 54, in the detail of free surface instabilities, is presented an increase of about $50 \%$ in the mean speed in the free surface region. The Figure 58 presents the contour of velocity curl in the fluid domain. According to the Figure 56 This curl intensity is similar to the induced by the tip blades. The Figure 57 presents the velocity curl isosurfaces in the fluid domain. An interaction between the tip vortex and the vorticity in the free surface seems to happen. However, is essential to highlight that it is not known at the present moment if such effect would happen in a real flow or if it is caused just by numerical instabilities.

Figure 55 - Streamlines for Open Channel Flow - TSR $=6$ - Inlet Velocity of $0.173 \mathrm{~m} / \mathrm{s}-$ View of the entire domain

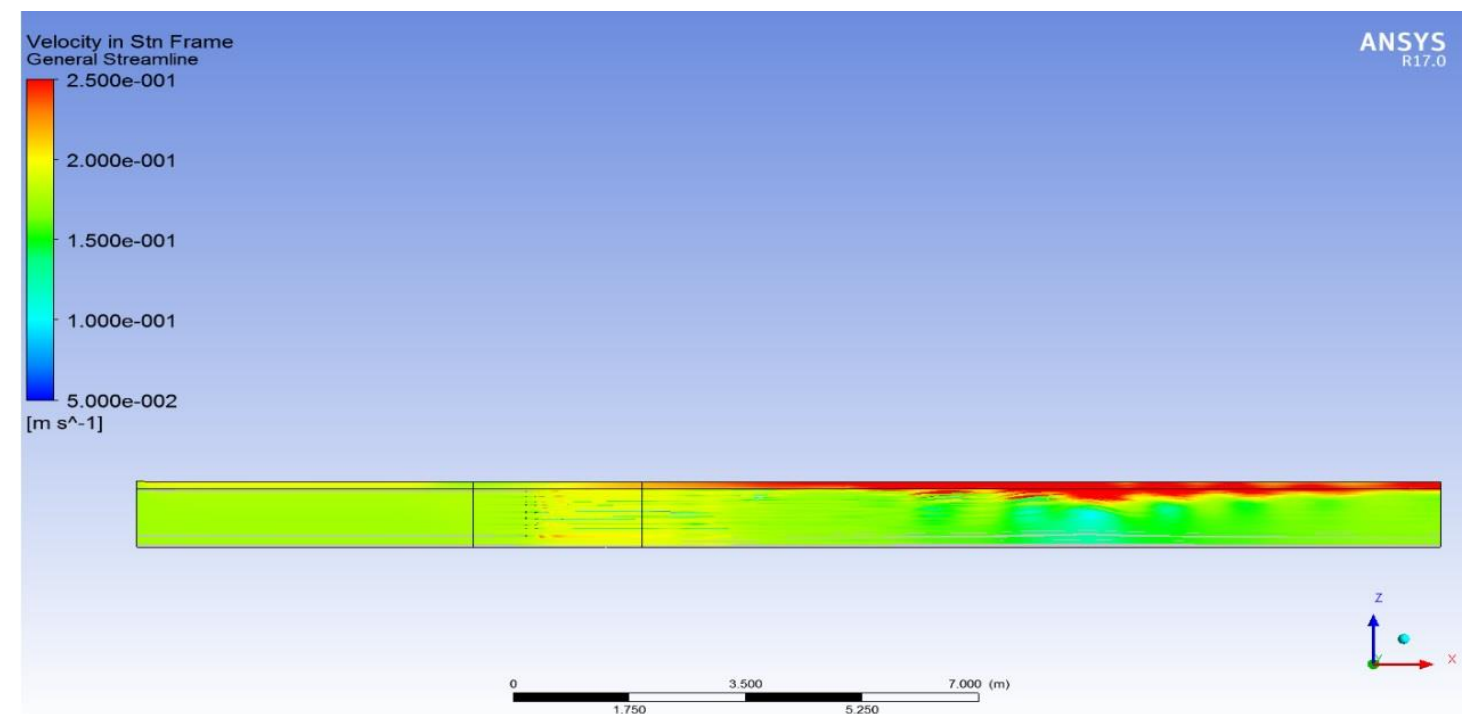


Figure 56 - Velocity Curl Contour- for Open Channel Flow - TSR $=6$ - Inlet Velocity of 0.173 $\mathrm{m} / \mathrm{s}$

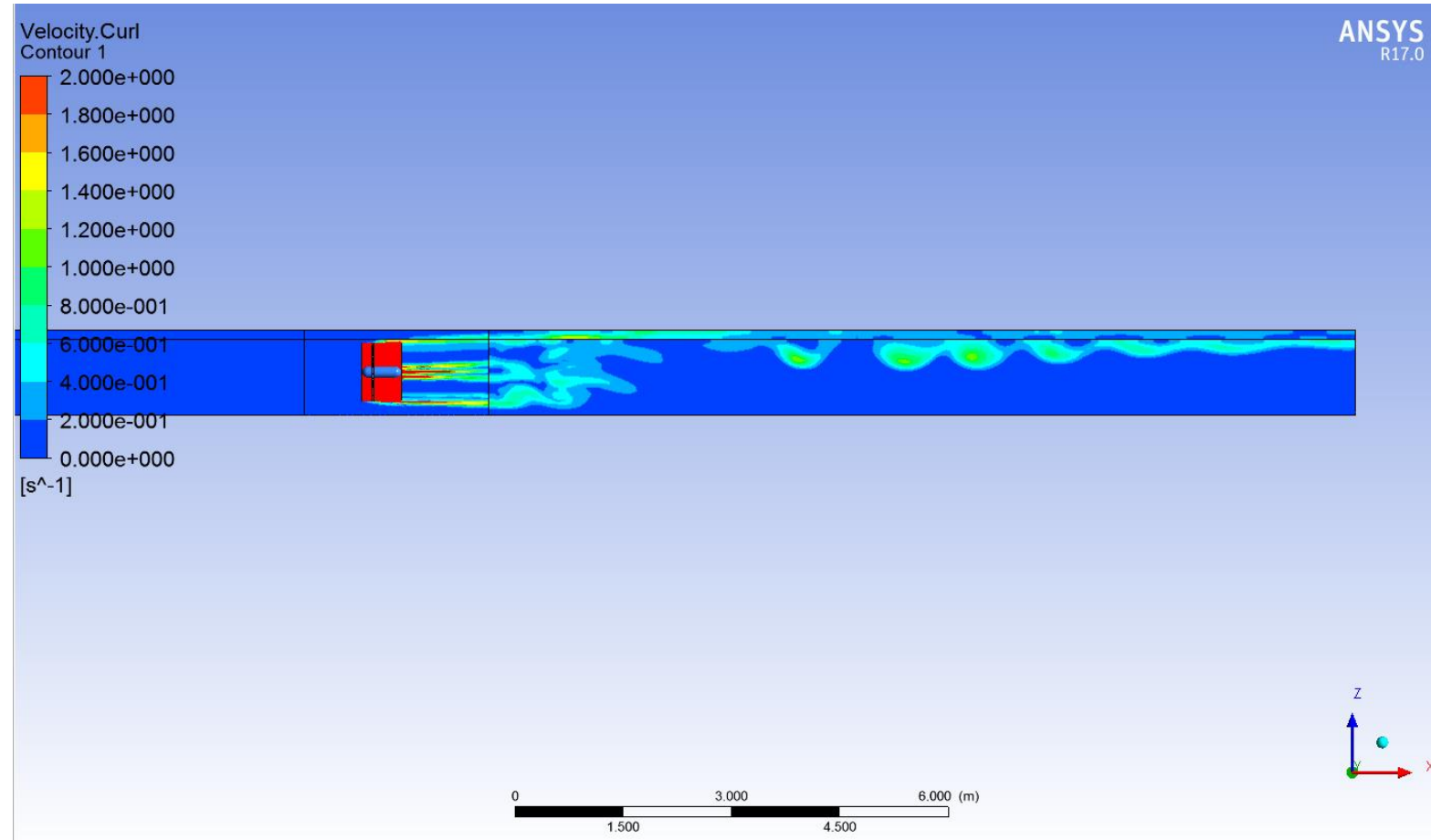

Figure 57 - Velocity Curl Isosurface- for Open Channel Flow - TSR $=6$ - Inlet Velocity of $0.173 \mathrm{~m} / \mathrm{s}$

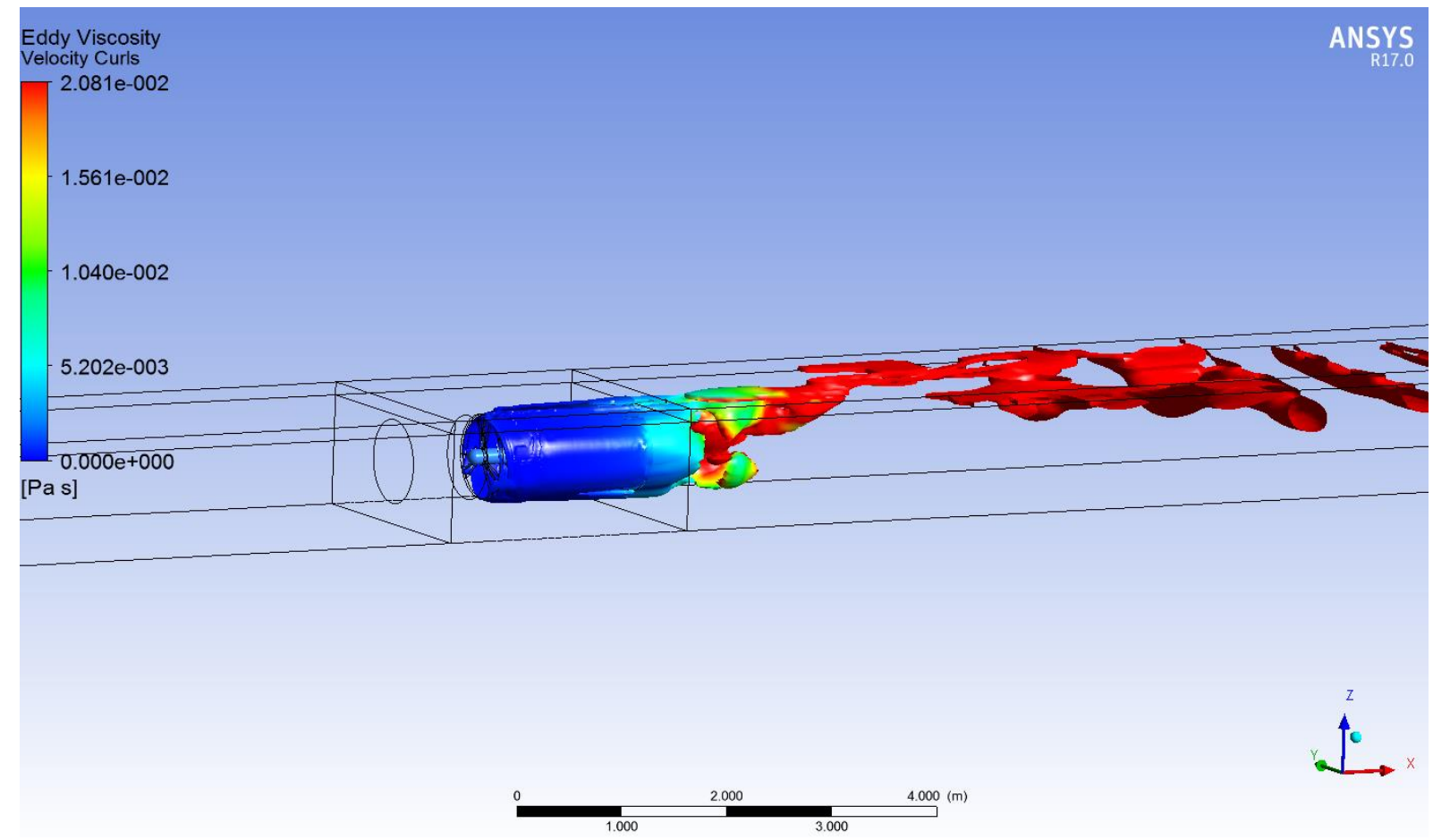


The vortices in the wake region are also seen in the Figure 58. The differences between the velocities in this region, with higher speed above and below turbine disk together with velocities $30 \%$ higher near to the turbine axis induces shear stress in the flow that makes the fluid particles rotate, starting the vertical formation. The wake is not symmetrical concerning the turbine center horizontal plane. It may occur due to the free surface, and it is also seen by the velocity curl contour, velocity curl isosurfaces and the streamlines.

The Figure 59 shows a uniform velocity profile with exception to the interface region between the static domain and Indeformable domain.

In the Figure 60the velocity distribution in the turbine disk is presented. High velocities in the blades suction side and low velocities in the blade pressure side are seen, which is expected since the main motion load is the lift produced in those regions that causes a torque that powers the turbine.

The downstream profile of velocity is presented from the Figure 61 to the Figure 64 . The first figure shows the velocity contour for a distance of 1 turbine disk diameter from the turbine disk plane. It is seen a velocity defect in the turbine disk region, which is already expected since it represents the wake region. There is an increase of the velocity close to the free surface above to the turbine disk.

Figure 58 - Velocity Contour - for Open Channel Flow - TSR $=6$ - Inlet Velocity of $0.173 \mathrm{~m} / \mathrm{s}$

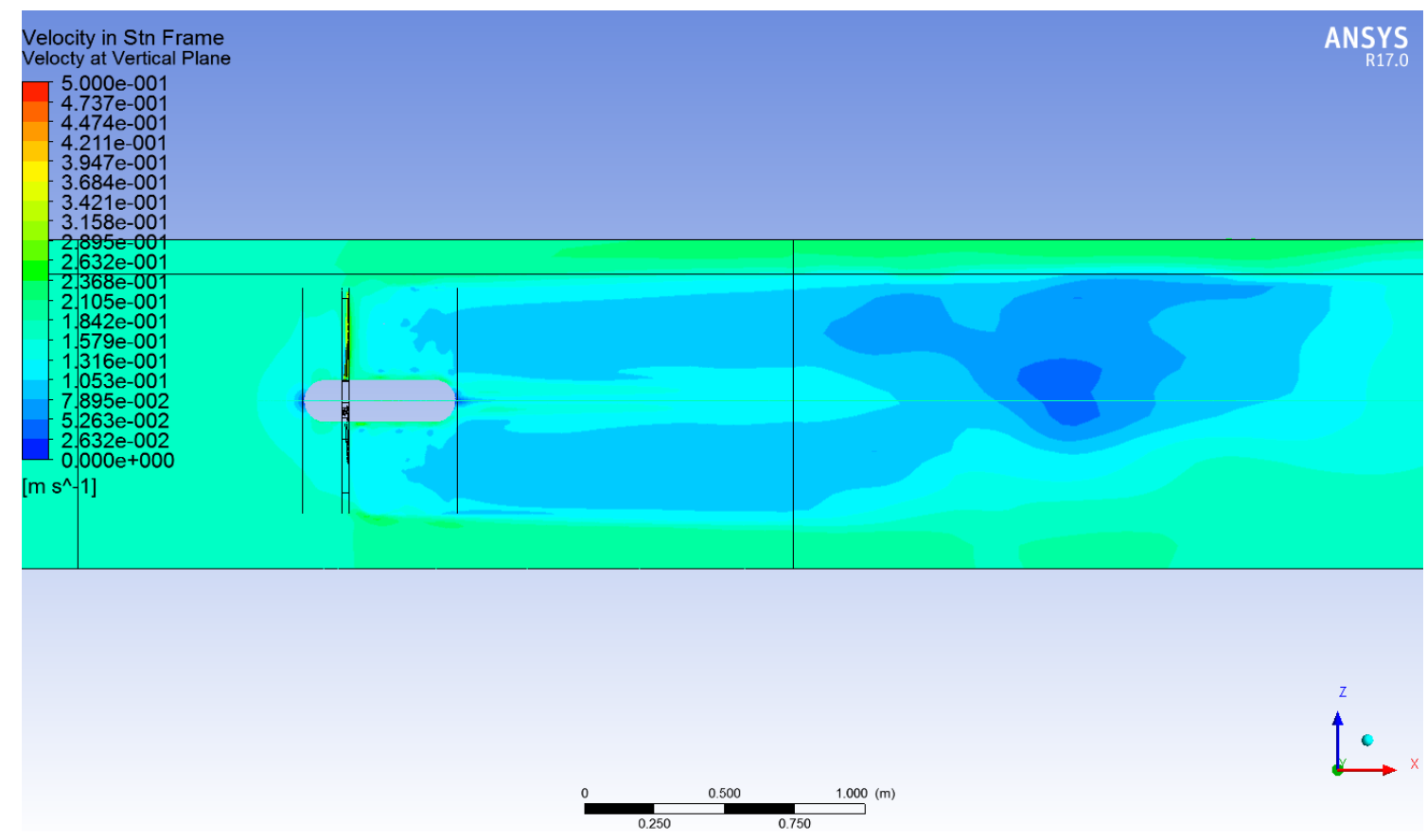


Figure 59 - Inlet Velocity Profile - Distance of 1 Diameter from Turbine Disk

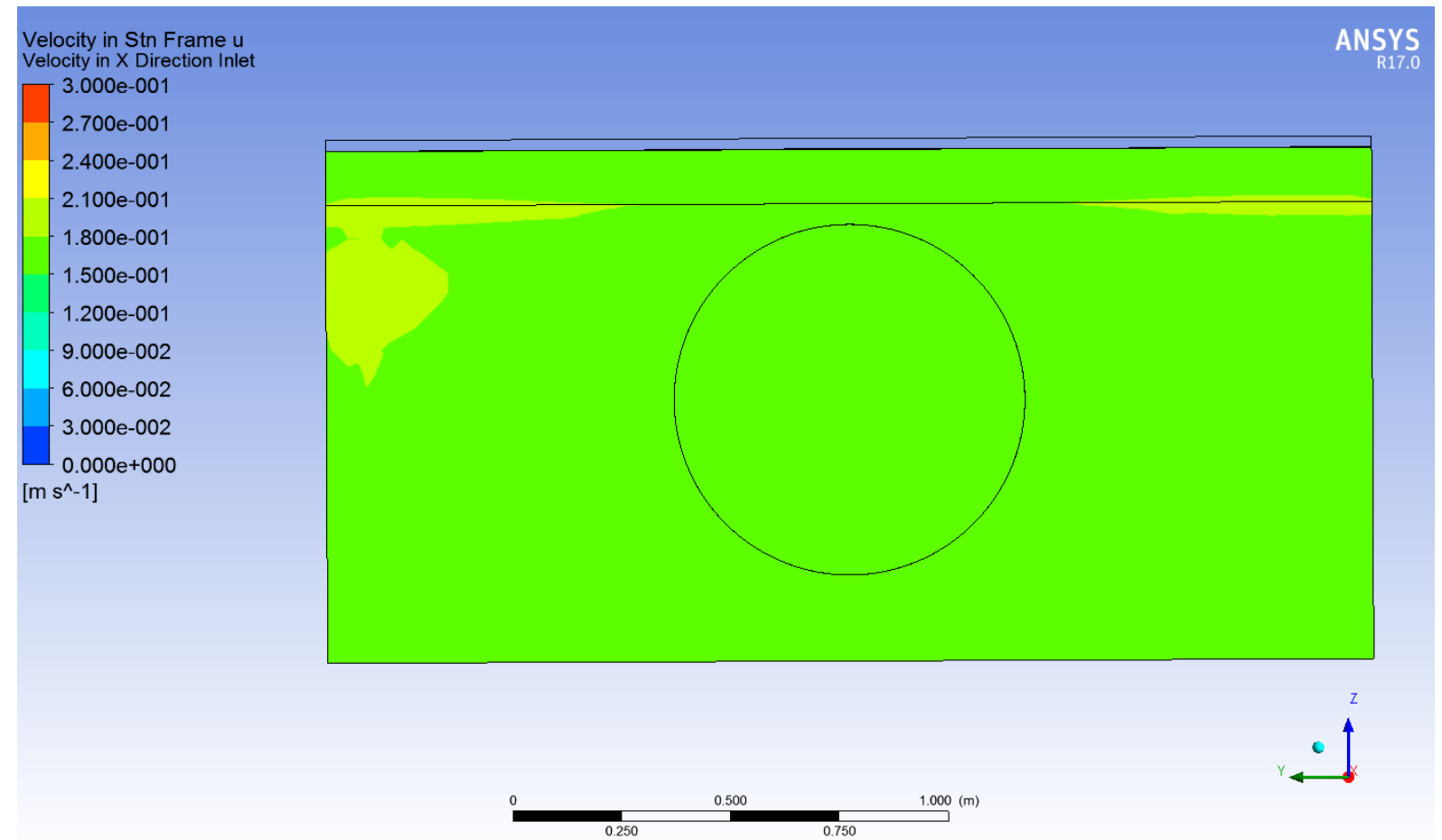

Figure 60 - Turbine Disk Velocity Profile

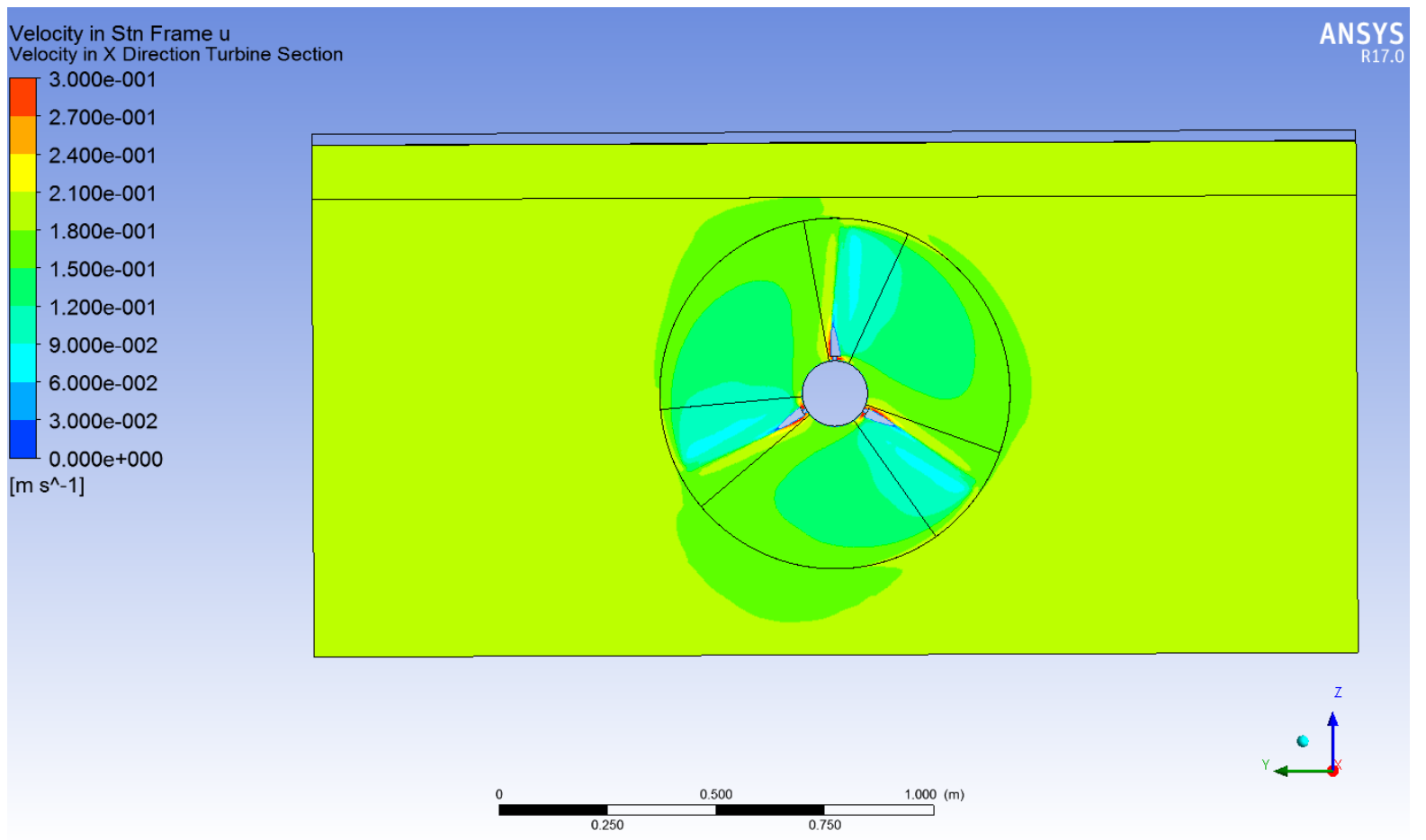


Figure 61 - Outlet Velocity Profile - Distance of 1 Diameter from Turbine Disk

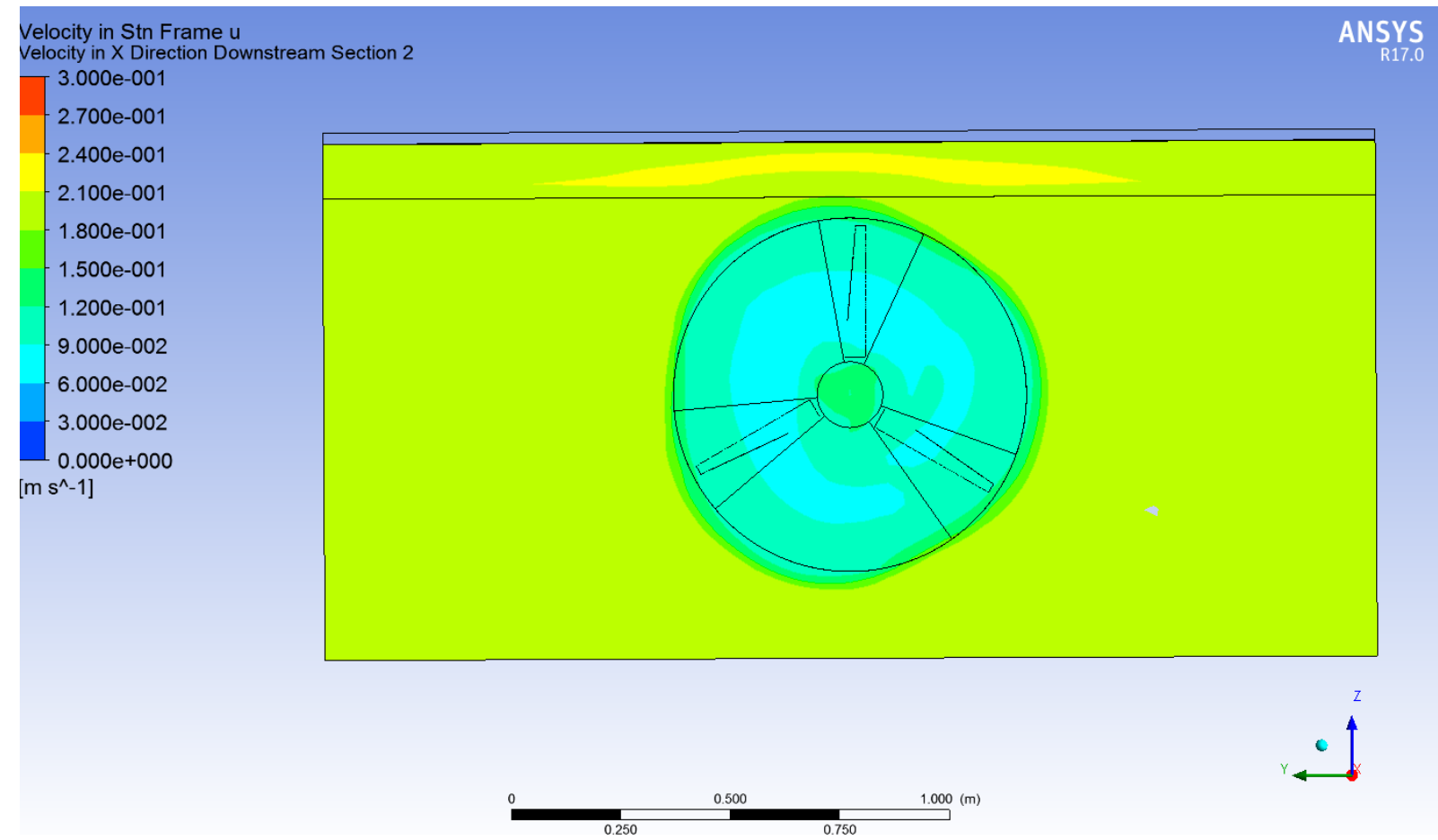

The Figure 62 presents the velocity distribution at a distance of 3 diameters from the turbine disk. The velocity in the turbine plane has returned to the inlet velocity but speeds $50 \%$ above inlet speed are presented in the free surface region.

Figure 62 - Outlet Velocity Profile - Distance of 3 Diameters from Turbine Disk

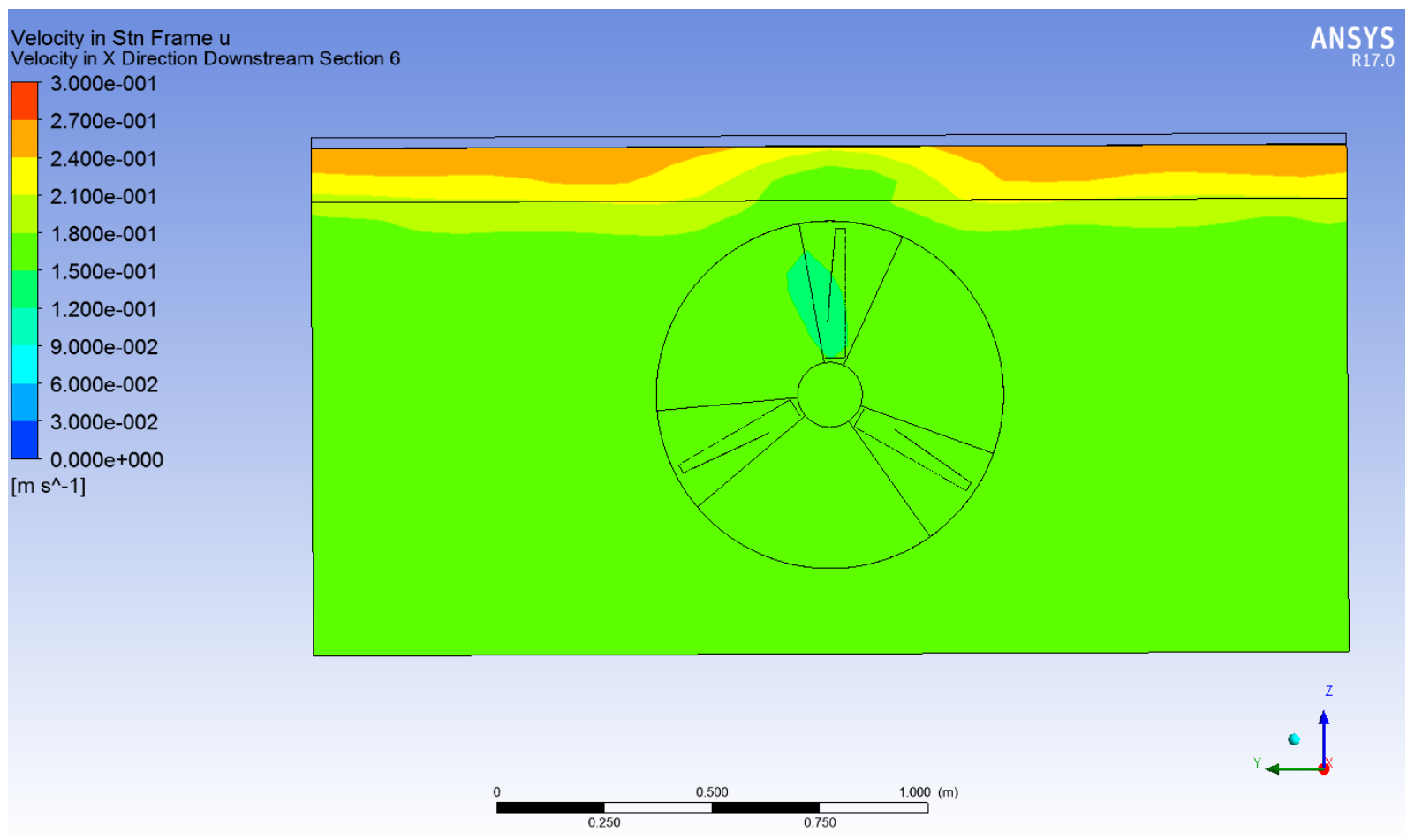


The Figure 63 presents the velocity distribution 6 diameters downstream from the Turbine Disk. There is a velocity level almost $100 \%$ above the inlet velocity. This region presents a high vorticity as discussed previously. The Figure 64 presents the velocity profile 10 diameters from the turbine disk. The velocity on the free surface region decreased but still 50\% above the inlet velocity.

Figure 63 - Outlet Velocity Profile - Distance of 6 Diameters from Turbine Disk

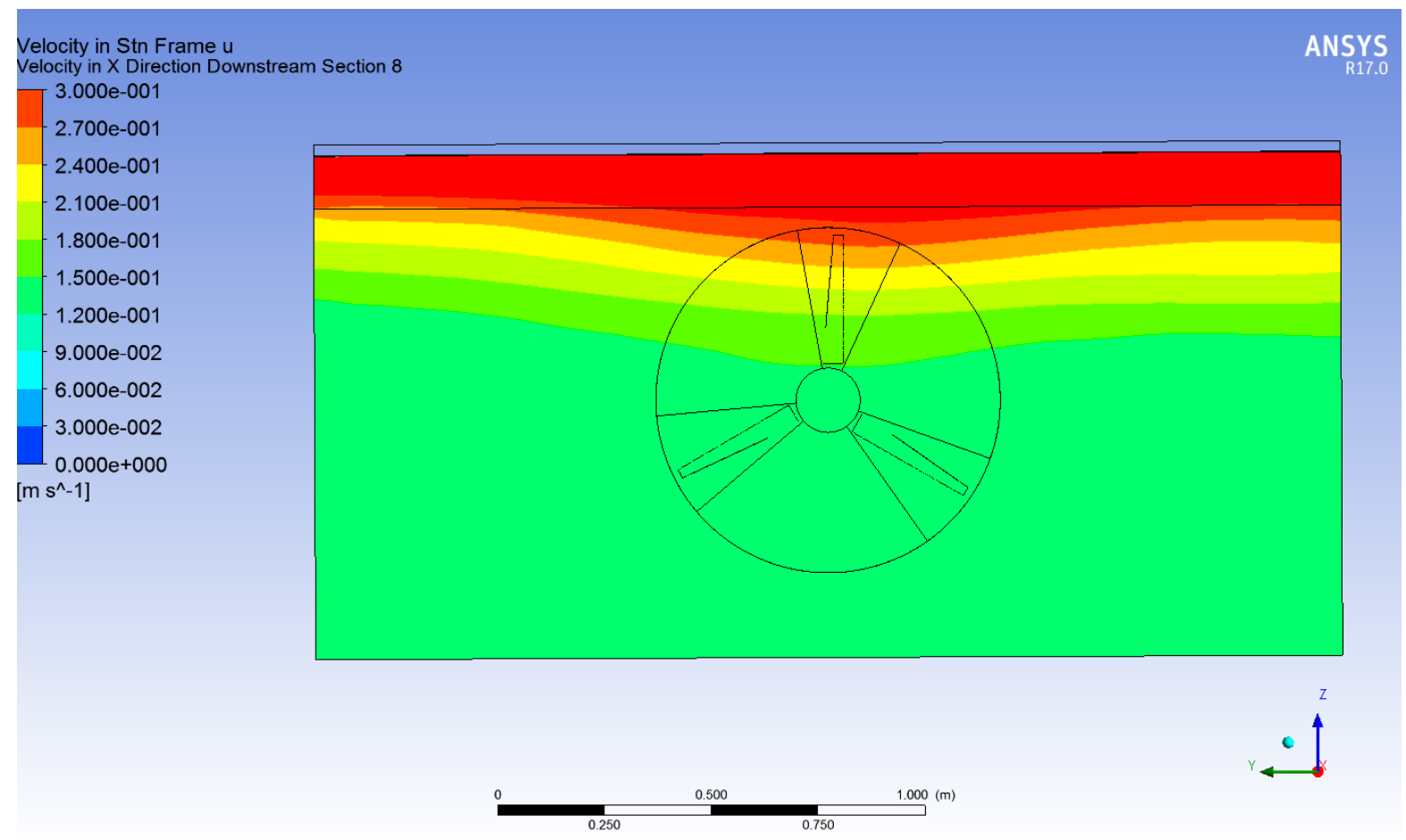

Figure 64 - Outlet Velocity Profile - Distance of 10 Diameters from Turbine Disk

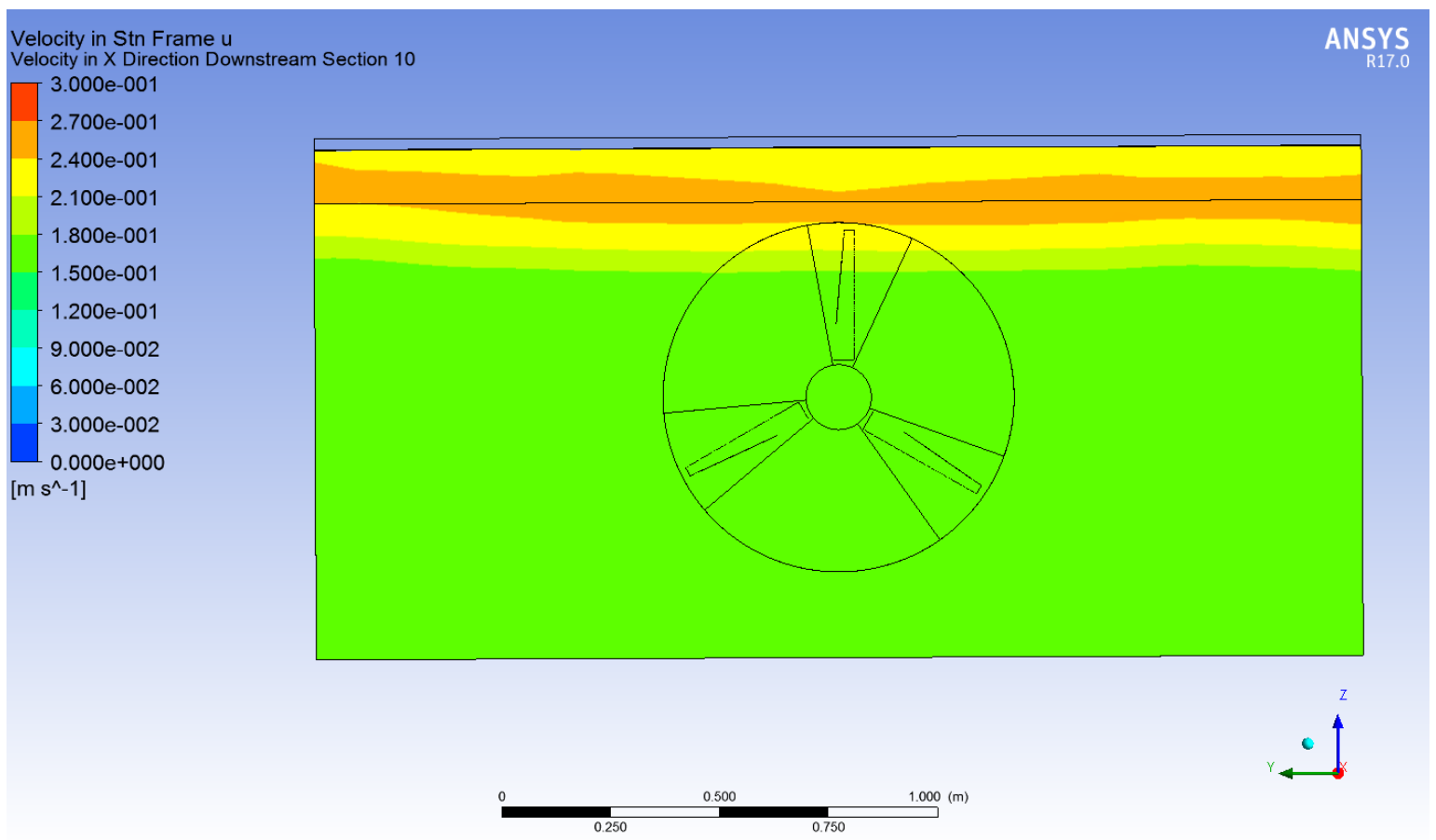




\subsubsection{Deformations in the Solid Model}

Figure 65 - Stresses in Z-Direction - TSR $=6$; Inlet velocity of $0.173 \mathrm{~m} / \mathrm{s}$

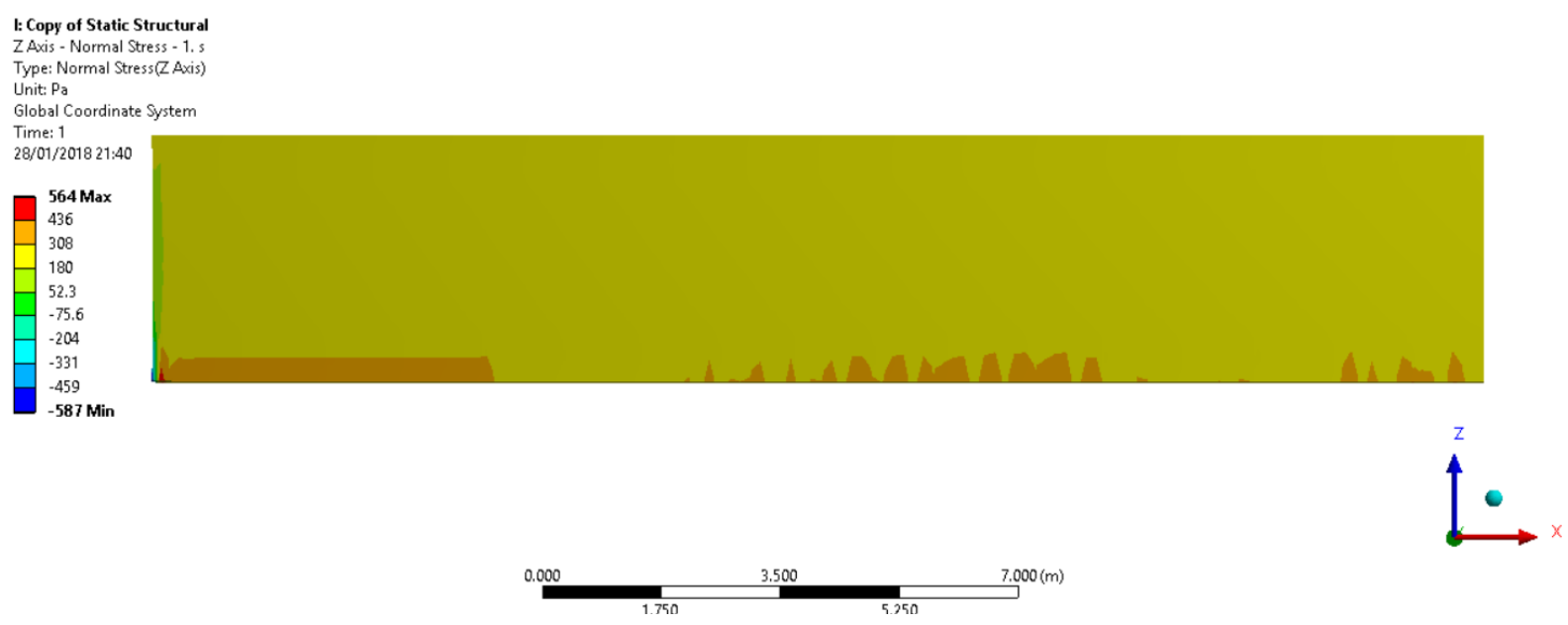

Figure 66 - Orthotropic Body deformation - TSR = 6; Inlet Velocity of $0.173 \mathrm{~m} / \mathrm{s}$

I: Copy of Static Structural

Directional Deformation

Type: Directional Deformation(Z Axis)

Unit: $\mathrm{mm}$

Global Coordinate System

Time: 1

28/01/201821:35
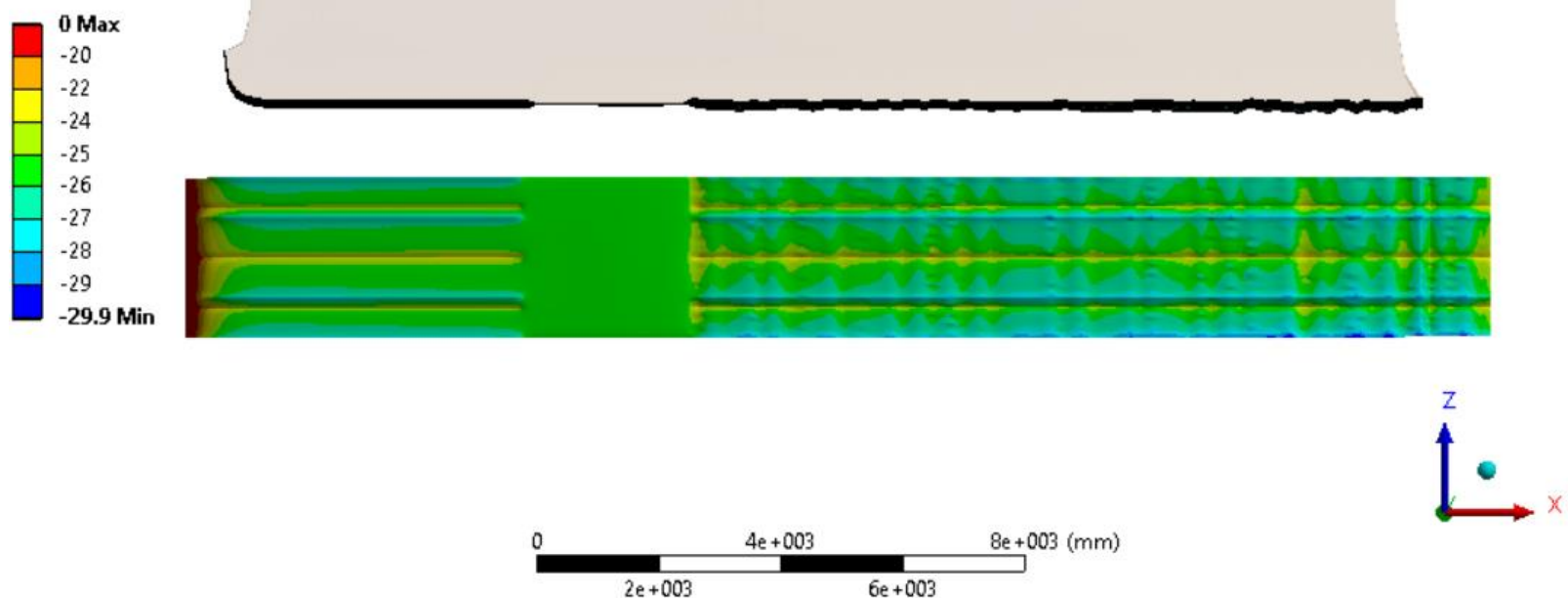

The Figure 65 shows the stresses in z-direction distribution and the Figure 66 shows the deformations in the free surface region. As expected, the negative deformations correspond to positive stresses on the body (traction), and the positive deformations correspond to negative stresses on the body (compression), which shows that the body assumes the spring behavior, necessary to this free surface modelling. 
An issue regarding to this model is related to the solid pre-stress. Since in the boundary conditions, the only specified pressure is the outlet, and the model initially assumes that the inlet deformation is zero, the definition of the solid pre-stress must make the inlet pressure equals to the solid pre-stress which is not directly defined in this model. Hence, for the model consistency, the outlet pressure was adjusted to the inlet pressure achieve the same pressure as the calculated for the pre-stress. However, for the subsequent coupling interactions between the solid and fluid models, the pressure difference between inlet and outlet changes, requiring an additional updated in the outlet pressure, which is not possible only using the ANSYS Workbench $17.0 \AA$. It caused an inlet deformation, shown in Figure 65. However, since the distance between the inlet and the turbine disk is $8000 \mathrm{~mm}$ and the deformation order is $25 \mathrm{~mm}$, the flow had become uniform before passing through the turbine.

None the less, for further simulations, a code must be implemented within the ANSYS CFX to make the outlet pressure iterative to guarantee the zero deformation at the flow inlet. 


\subsection{Comparison Between the CFD Models}

As expected, the confined domain presented the higher power coefficient, by comparison with the open domain and open channel domain. The confinement guarantees that a higher portion of fluid passes through the turbine disk, and thus, a higher kinetic energy flow passes through the turbine disk too.

The calculated power coefficient for the confined flow was $11.5 \%$ higher than the calculated for open domain flow. The prediction according to (Garret \& Cummins, 2007) of $45 \%$ did not confirm, which is expected due to the simplicity of the theory, which only analyses the flow in one direction, neglecting the geometrical particularities of the turbine and other flow characteristics.

The open channel model, for the case with $0.173 \mathrm{~m} / \mathrm{s}$ inlet velocity presented the same power coefficient of the open domain flow, however, due to the difference of the Reynolds number between the two simulations, this comparison cannot be precisely made, since the effects of the Reynolds number are not known for this case. However, the instabilities in the wake, presented for the open channel model, indicated that the model did not fully converge. The graphic comparing the power coefficients in the different boundary conditions is shown in Figure 67.

Figure 67 - Comparison between the power coefficients between the different boundary conditions

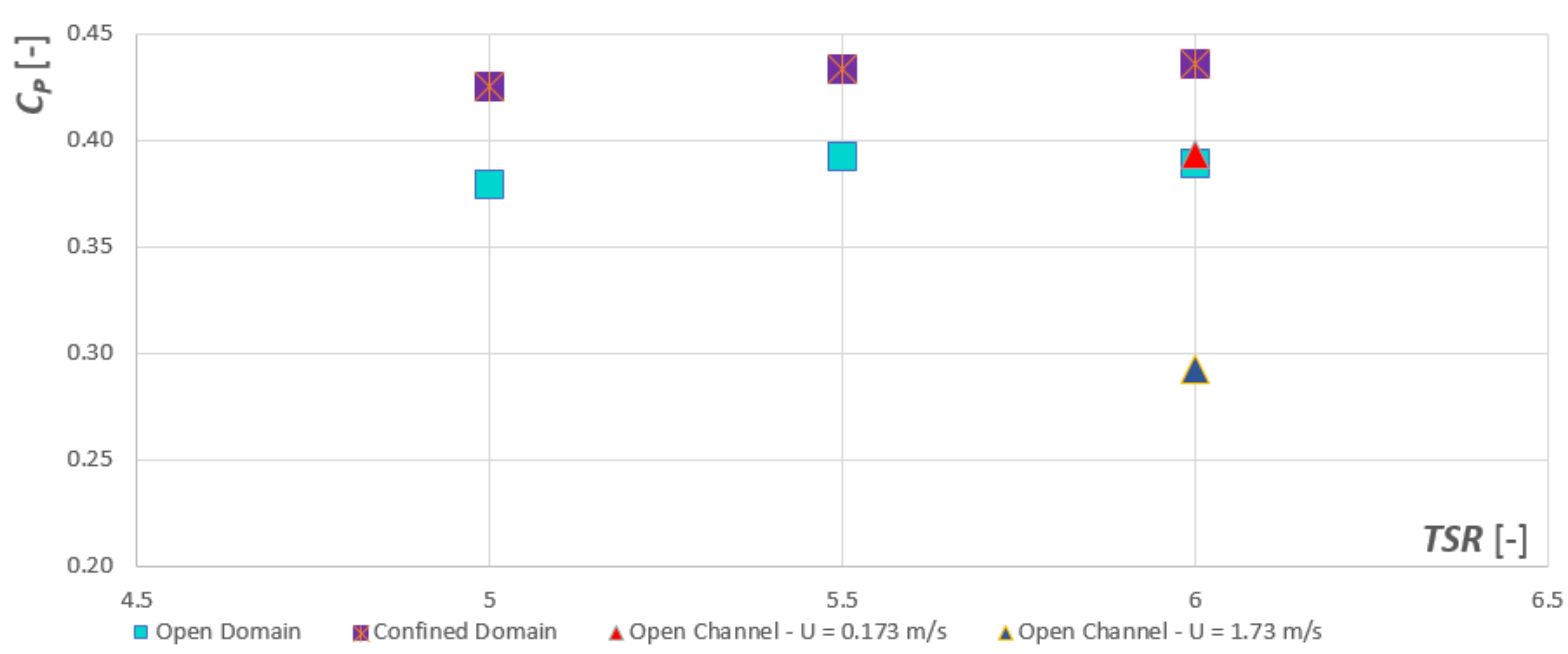


The data of thrust coefficient, shown in Figure 68, indicates that the confinement increases the axial thrust of the turbine, which causes higher pressure differences than the open flow and the open channel cases. The average difference between open flow and confined domain cases is $8 \%$, lower than the difference found for power coefficient measurements. It indicates to a higher efficiency of the turbine with the flow confinement.

For the open channel cases, both simulations led to a lower thrust coefficient, which is inconsistent with the actuator disk theory. For the simulation with lower inlet velocity, the result almost matched with the open domain case. However, according to the actuator disk theory, the increase of Froude number causes an increase in the power coefficient, that is shown in Figure 69.

Figure 68 - Comparison between the thrust coefficients between the different boundary conditions

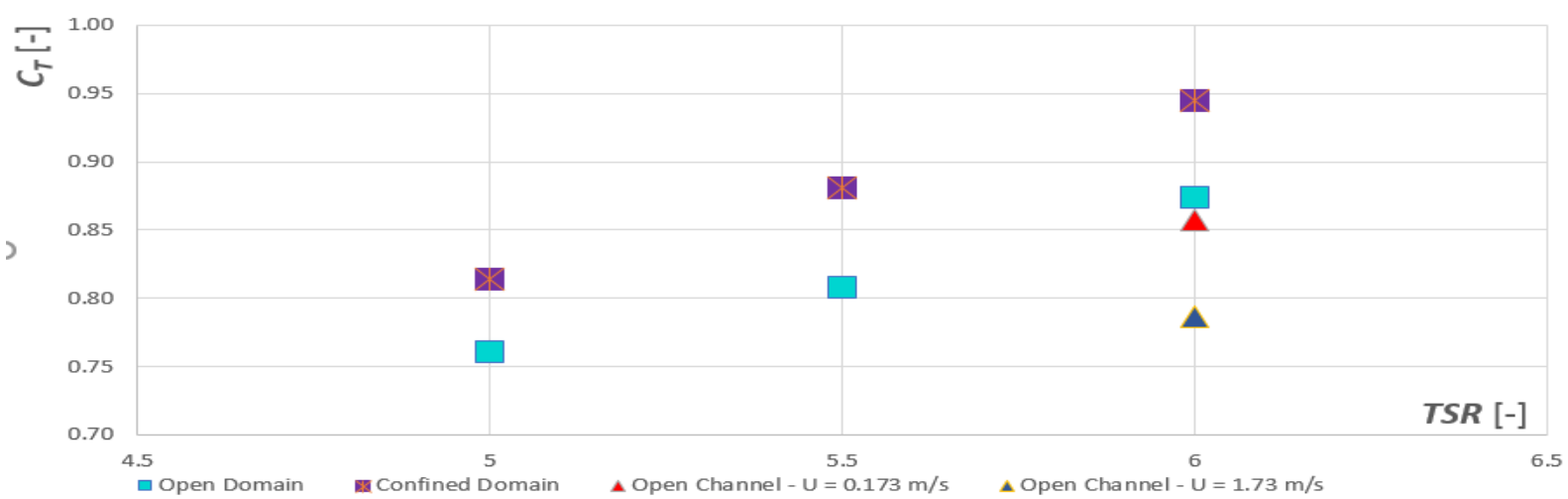

Figure 69 - Power coefficient in function of Froude Number according to the actuator disk theory for an open channel

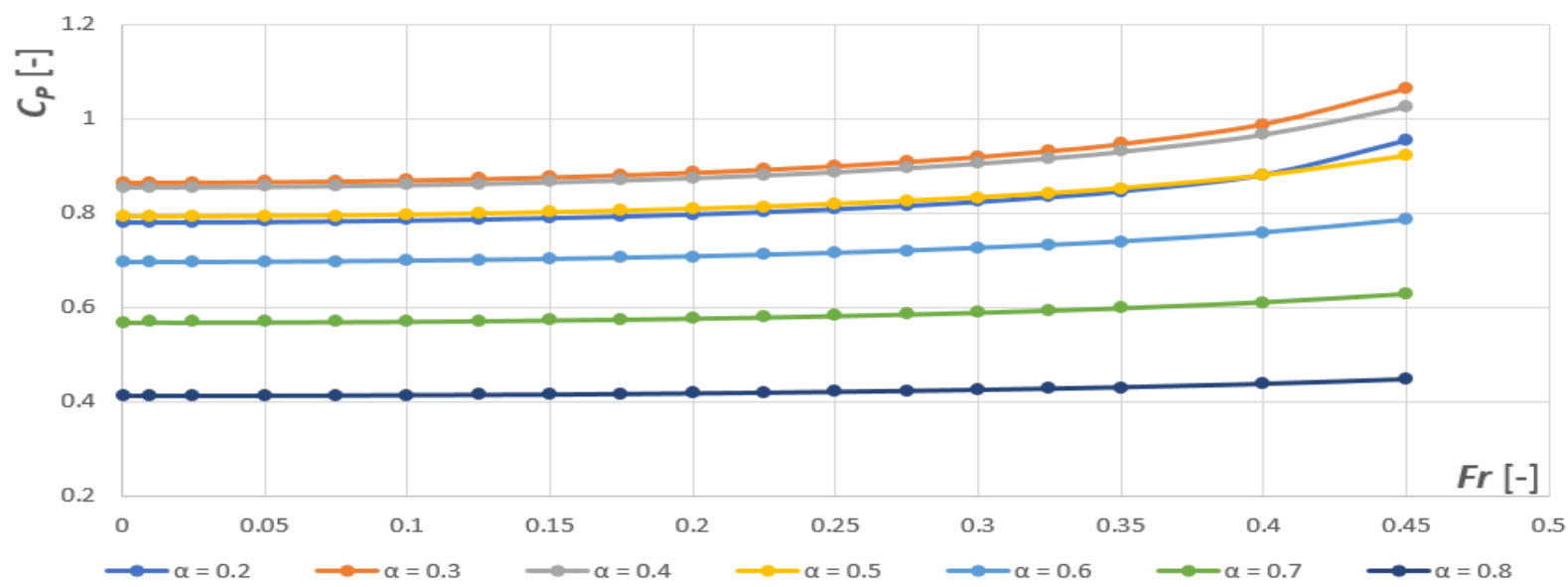




\section{Chapter 9. Conclusion}

The comparison of the $\boldsymbol{C}_{\boldsymbol{P}}$ and $\boldsymbol{C}_{\boldsymbol{T}}$ curves between experimental methodology and computational results for the open domain geometry showed that the CFD has a good agreement with the experiments, validating the computational method and its features, such as the mesh density and turbulence models used. The curves obtained through CFD presented the same shape of the curves obtained through experiments, however, underpredicted the generated power and overpredicted the thrust, which is already expected according to the literature.

The analysis with the confined domain showed that the blockage increases the generated power which agrees with the theoretical predictions. The wake length is higher, which could decrease a tidal farm total generation since the mean velocity in the wake region is lower than the inlet velocity. A computational analysis varying the blockage ratio is missing to evaluate the impact of this variable on the hydrodynamic performance of an axial tidal current turbine.

The confinement has a low influence in the flow separation pattern, which is highlighted by the comparison between the wall shear stress plots. Additional investigations must be performed to analyze the influence of the boundaries in the flow through the blades which increases the generated power. With the current simulations, any statement could be made regarding to the attack angles and the difference between this parameter comparing the different boundary conditions used in this work.

Regarding the free surface model using an elastic free surface, the results for the original Reynolds and Froude number was not accurate and did not converge, due to the high initial deformation presented, nevertheless, the thrust calculation showed missprediction about $16 \%$ if compared with the confined domain.

The non-convergence of the elastic free surface for the initial inlet velocity occurred due to the high initial deformations, compared with the cross-sectional area. These deformations induced high-velocity gradients in the free surface region which caused high pressures, making the algorithm became unstable. Further development on this 
algorithm shall include a gradual deformation of the free surface in order to avoid such problems.

For the free surface methodology analysis, the simulation with $10 \%$ of inlet velocity was performed providing a good approach for both torque and thrust, and considering that the Froude number was decreased with the inlet velocity, it is expected that the simulation for the original Froude number would provide a higher power and thrust values.

The analytical models, analyzed in section 6.1 , showed that the proposed model is physically consistent, providing the same balance between potential and kinetic energy as the gravity-based models, as the VOF methodology. Moreover, the CFD analysis with the elastic free surface model provided feasible results, which contributes to validate the potential of the methodology to analyze free surface flows. 


\section{Chapter 10. Proposals for complementary works}

Even this work is focused on the hydrodynamic analysis of tidal current turbines, the methodology implemented for free surface analysis has shown to be promising since it is computationally efficient if compared with VOF approach and presents fewer problems related to numerical diffusion in the free surface region. Therefore, the complementary works cover both areas.

\subsection{Tidal current turbines}

This work evaluated a single turbine subjected to different boundary conditions. All performed analysis was made in a steady state condition. Therefore, analyses involving transient analyses such as the pressure fluctuations due to rotational effects are necessary to evaluate the order of the power fluctuations in power generated. The simulation of symmetry and anti-symmetry conditions is not found in the literature.

The more recent experimental analysis evaluated the interaction between two turbines placed on the same axis. A numerical analysis of such problem is interesting to evaluate the interaction mechanisms and assessing parameters as the difference of rotation between the two turbines and the rotation inversion in the total generated power.

Several analyses of tidal farm generation optimization are found in the literature, indicating that most of the researches see more benefits in optimizing the whole farm than a single turbine. However, most CFD optimization techniques assume a constant $\boldsymbol{C}_{\boldsymbol{T}}$ and $\boldsymbol{C}_{\boldsymbol{P}}$ for all turbines, neglecting the influence of inlet velocity on such parameters. 
Therefore, the following works are suggested for the theme development continuity:

- Transient analysis of tidal current turbine confined in a closed channel with high blockage and transient analysis of tidal current turbine in an open channel;

- Transient BEMT method involving the interaction between the blade and the free surface;

- Steady State and Transient analysis of two tidal current turbines placed on the same axis;

- Optimization analysis of a tidal current farm using $C_{P}$ and $C_{T}$ calibrated with a real turbine experimental and numerical analysis.

\subsection{Free surface analysis}

The elastic free surface used during this work produced accurate results for the tidal current turbine analysis, and the analytical development showed the physical consistency of such methodology that provides energy conservation and even for transient analysis, could have its density adjusted to produce feasible results. However, since in the current bibliography, it was not found any similar methodology, it is needed a more profound analysis.

Furthermore, only parameters such as thrust and torque were evaluated during the CFD analysis. Hence, it is needed analysis comparing the flow pattern and the velocity profiles with the measured experimentally and with the obtained through VOF methodology with the free surface.

Besides of that, during the process of Fluid-Structure coupling, it issues with convergence, mainly due to the mesh deformation algorithm were found. The standard method from ANSYS CFX® is based on displacement diffusion, and for domains with small volumes, this could cause negative volumes, being physically and computationally infeasible, stopping the calculation routine. 
Since more validation cases, theoretical development and a consistent computational implementation still needed, it is proposed the following works:

- Evaluate the impacts of this boundary condition in a viscous fluid flow analytically;

- Analyse the velocity profiles for flow around a circular cylinder with steadystate and transient analysis;

- Analyse the flow through a circular cylinder piercewising the free-surface;

- Couple the elastic free surface methodology with a more robust mesh deformation algorithm, designed for large deformations. An elastic mesh is proposed in (Battaglia et al., 2004) for solving a free surface problem, showing a trend of the solution of such problem.

- The study of solution stability of this methodology. 


\section{Chapter 11. References}

Afgan et al. (2012). Large-Eddy Simulation of a 3-bladed horizontal axis tidal stream turbine: comparisons to RANS and experiments. Turbulence, Heat and MAss Transfer(7), 1 - 13.

Andritz Hydro. (s.d.). Hydro News 27 - Tidal Energy. Acesso em 04 de January de 2018, disponível em https://www.andritz.com/hydro-en/hydronews/27/hynews-27-03-tidal-energy-hydro

ANSYS Inc. (2016). Ansys 17.0 Users Guide. ANSYS Inc.

Bahaj, A., W.M.J., B., \& McCann, G. (2007). Experimental verifications of numerical predictions for the hydrodynamic performance of horizontal axis marine current turbines. Renewable Energy, 2479-2490.

Barnsley M.J., W. J. (1990). Final report on 2nd phase of development and testing of a horizontal axis wind turbine test rig for the investigation of stall regulation aerodyamics. Carried out under ETSU Agreement E.5a/CON5103/1746 .

Battaglia et al. (2004). Numerical Simulation of Transient Free Surface Flows Using a Moving Mesh Technique. ENIEF.

Betz, A. (1920). Das Maximum der theoretisch möglichen Ausnützung des Windes durch Windmotoren. Zeitschrift für das gesamte Turbinenwesen.

Bir et al. (2011). Structural Design of a Horizontal-Axis Tidal Current Turbine. ASME 30th International Conference on Oceanic Offshore and Artic Engineering. Roterdam.

Biskup et al. (2014). mulation of Fluid-Structure Interaction on Tidl Current Turbines Based on Coupled Multibody and CFD Methods. Journal of Ocean and Wind Energy, 1(2), 119 - 126.

Blackmore et al. (2014). Influence of turbulence on the wake of a marine current turbine simulator. Southampton: Royal Society Publishing. 
British Hydro. (14 de October de 2009). Fonte: British Hydro Org: http://www.britishhydro.org/downloads/La\%20Rance-BHA-Oct\%202009.pdf

Consul, C. (2011). Cross Flow Turbine. In: Hydrodynamic Analysis of a Tidal CrossFlow Turbine. Trinity: Worscester College.

Empresa de Pesquisas Energéticas. (12 de November de 2017A). Leilão de Energia A4-2017. Fonte: Empresa de Pesquisas Energéticas: http://www.epe.gov.br/leiloes/Documents/Resumo\%20Cadastramento\%20\%20Leil\%C3\%A30\%20A-4\%202017\%20(v3).pdf

Empresa de Pesquisas Energéticas. (12 de November de 2017B). Leilão de Energia A6/2017. Fonte: Empresa de Pesquisas Energéticas: http://www.epe.gov.br/leiloes/Documents/Resumo\%20Cadastramento\%20\%20Leil\%C3\%A3o\%20A6\%202017\%20(v3).pdf

Estafahani, J., \& Karbasian, H. (2012). Optimizing the Shape of Rotor Blades for Maximum Power Extraction in Marine Current Turbines. International Journal of Automotive and Mechanical Engineering, 6, 722-729.

Falnes, J. (2007). A Review of wave-energy extraction. Marine Structures 20.

Ferziger, J., \& Peric, M. (2002). Free-Surface Flows. In: Computational Methods for Fluid Dynamics (pp. 381 - 397). Springer.

Forinash, C., \& DuPont, B. (2016). Optimization of floating offshore wind energy systems using an extended pattern search method. Proceedings of the ASME 2016 35th International Conference on Ocean, Offshore and Artic Engineering. Busan.

Galloway et al. (2011). Experimental and numerical results of rotor power and thrust of tidal turbine operating at yaw and in waves. World Renewable Energy Congress 2011. Linköping.

Garret, C., \& Cummins, P. (2007). The efficiency of a turbine in a tidal channel. Journal of Fluid Mechanics, 588, 243 - 251. 
Gebreslassie et al. (2012). Comparison of Analytical and CFD Modelling of the Wake Interactions of Tidal Turbines. Exeter: College of Engineering, Mathematics and Physical Sciences from University of Exeter.

Gelfand, I., \& Fomin, S. (1963). The General Variation of a Functional. In: Calculus of Variations (pp. 54 - 66). Prentice Hall.

Green Rhyno Energy. (16 de May de 2018). Wind Energy and the Environment. Fonte: Green Rhyno Energy: http://www.greenrhinoenergy.com/renewable/wind/wind_environment.php

Hansen, J. (2015). Ice melt, sea level rise and superstorms. Atmospheric, Chemistry and Physics Discussions, p. 20062.

Houlsby et al. (2007). Application of Linear Momentum Actuator Disc Theory to Open Channel Flow. Oxford: University of Oxford - Department of Engineering Science.

Hughes, T. (2000). Fundamental Concepts - A Simple one-dimensional boundaryvalue Problem. In: The Finite Element Method - Linear Static and Dynamic Finite Element Analysis (pp. 1 - 56). New York: Dover Publications Inc.

International Energy Agency. (2017). Global Electrical Vehicules Outlook 2017. International Energy Agency.

IRENA. (2014). IRENA Ocean Energy Technology Brief 3.

Jones, W., \& Launder, B. (1972). The prediction of laminarization with a two-equation model of turbulent. Journal of Heat and Mass Transfer 15, 301-314.

K Water. (s.d.). K Water. Acesso em 18 de December de 2017, disponível em http://english.kwater.or.kr/eng/busi/project03Page.do?s_mid=1192

Kim, J., \& Moser, R. (1987). Turbulence statistics in fully developed channel flow at low Reynolds Number. Journal of Fluid Mechanics 177, 133-166.

Kulkarni et. al. (2016). Computational Fluid Dynamic (CFD) Mesh Independency Study of a Straight Blade Horizontal Axis Tidal Turbine. Birmingham: Birmingham City University. 
Lai, M., Krempl, E., \& Ruben, D. (2010). Linearly Elastic Solid. In: Introduction to Continuum Mechanics (pp. 204 - 207). Elsevier.

Lamb, H. (1879B). Waves due to a Submerged Cylinder. In: Hydrodynamics (pp. 410 - 413). Cambridge University Press.

Lanczos, C. (1949). Mechanics of the Continua. In: The variational principles of mechanics (pp. 365 - 369). University of Toronto Press.

Launder, B., \& Spalding, D. (1974). The numerical computation of turbulent flows. Computational Methods Applied to Mechanical Engineering, 269-289.

Lloyd et al. (2011). Unsteady CFD of a Marine Current Turbine using OpenFOAM with Generalized Grid Interface. University of Southampton.

Maliska, C. (2014). Transferência de Calor e Mecânica dos Fluidos Computacional 2ed. Rio de Janeiro: LTC.

Mason-Jones et al. (2012). Non-dimensional scalling of tidal stream turbines. Energy, $444,820-829$.

Masters et al. (2011). A robust blade element momentum theory for tidal stream turbines including tip and hub loss corrections. Journal of Marine Engineering and Technology, 11, 25 - 35.

Masters et al. (2015). A Comparison of Numberical Modelling Technique for Tidal Stream Turbine Analysis. Energies, 8, 7833-7853.

Mattavo, F., \& Assi, G. R. (2017). Free Surface Modeling Employing a Two-Way Fluid Structure Interaction Approach for the Flow Around a Circular Cylinder. COBEM 2017. Curitiba.

Meirovitch, L. (2001). Distributed Parameters System: Exact Solutions. In: Fundamentals of Vibrations (pp. 374 - 464). McGraw Hill.

Menter, F. (1992). Improved Two-Equation k- $\omega$ Turbulence Model for Aerodynamic Flowx. California: NASA.

Ministério de Minas e Energia do Brasil. (2017). Capacidade instalada de geração de energia no Brasil e no Mundo. 
Morris et al. (2016). Evaluatio of the swirl characteristics of a tidal stream turbine wake. International Journal of Marine Energy, 14, 198 - 214.

Munson et. al. (2004). Semelhança, Análise Dimensional e Modelos. In: Fundamentos da Mecânica dos Fluídos - Tradução da quarta edição americana (pp. 344 - 397). Edgard Blucher.

Munson, B., Young, D., \& Okiishi, T. (2002). Fundamentos da Mecânica dos Fluidos Tradução da 4a edição americana. São Paulo: Blucher.

Newman, J. (1977). Marine Hydrodynamics. Massachusetts: MIT Press.

Newman, J. (1977-A). Marine Hydrodynamics. In: J. Newman, Marine Hydrodynamics (pp. 237 - 327). Massachusetts: MIT Press.

Pantankar, S. (1980). Numerical Heat Transfer and Fluid Flow. Hemisphere Publishing Corporation.

Peiró et al. (2007). Modelling of free surface proximity and wave induced velocities around a horizontal axis tidal stream turbine. Proceedings of the 7th European Wave and Tidal Energy Conference. Porto.

Piggott et al. (2014A). Tidal turbine array optimisation using the adjoint approach. Renewable Energy, pp. 658 - 673.

Piggott et al. (2014B). A hierarchy of approaches for the otpimal design of tidal turbine farms. 5th International Conference on Ocean Energy. Halifax.

Pinón et al. (2012). Numberical Simulation of the ake of marine current turbines with a particle method. Renewable Energy, 46, 111 - 126.

Pinón et al. (2014). Experimental study of the turbulence intensity effects on marine current turbines behaviour. Part One: Single Turbine. Renewable Energy, 66, $729-746$.

Pinón et al. (2014B). Experimental study of the turbulence intensity effects on marine current turbines behaviour. Part II: Two interacting turbines. Renewable Energy, 68, 876 - 892. 
Pope, A. (1951B). The Juokowski Transformation. In: A. Pope, Basic Wing and Airfoil Theory (pp. 76-99). New York: McGraw Hill.

Pope, S. (2000). Turbulent Flows. Cambridge: Cambridge University Press.

Profito, F. (2015). On the Development of Advanced Texhniques for MixedElastohydrodynamic Lubrification Modelling of Journal and Sliding Bearing System. São Paulo.

Rao, S. (2008). Sistemas Contínuos. In: Vibrações Mecânica - Quarta Edição (pp. 272 - 304). Pearson Prentice Hall.

Reichl et al. (2005). Flow past a cylinder close to a free surface. Journal of Fluid Mechanics, 533, 269 - 296.

Rhie, C., \& Chow, W. (1982). A numerical study of the turbulent flow past an isolated airfoil with trailing edge separation. 3rd Joint Thermophysics, Fluids, Plasma and Heat Transfer Conference. St Louis.

Salter et al. (2002). Power conversion mechanisms for wave energy. Journal Engineering for the Maritme Environment, Vol 216, 1 - 27.

Salvatore et. al. (8 de May de 2018). Marine Turbine Hydrodynamics by a Boundary Element Method with Viscous Flow correction. Journal of Marine Science and Engineering, pp. 1-30.

Sharma et al. (2013). Characteristics of the turbulence in the flow at a tidal stream power site. Auckland: Royal Society Publishing.

Souza, Z. (2011). Perda de energia por fuga. In: Z. Souza, TOMO IV - Projeto de Máquinas de Fluxo - Turbinas Hidráulicas com rotores axiais (p. 116). Rio de Janeiro: Interciência.

Stansby, P., \& Stallard, T. (19 de February de 2016). Fast optimisation of tidal stream turbine positions for power generation in small arrays with low blockage based on superposition of self-similar far-wake velocity deficit profiles. Renewable Energy, pp. 366 - 375. 
Tatum et al. (2016). CFD Modelling of a tidal stream turbine subjected to profile flow and surface gravity effects. International Journal of Marine Energy(15), 156 173.

The Guardian. (17 de July de 2008). First tidal power turbine gets plugged in. Acesso em 04 de January de 2018, disponível em https://www.theguardian.com/environment/2008/jul/17/waveandtidalpower.ren ewableenergy

TidalEnergy Today. (17 de February de 2015). Tidal Energy Today. Acesso em 20 de october de 2017, disponível em http://tidalenergytoday.com/2015/02/17/estimate-of-global-potential-tidalresources/

Timoshenko, S. P., \& Goodier, J. (2010). Theory of Elasticity 3rd Edition. In: Theory of Elasticity 3rd Edition. McGraw Hill.

U.S. Energy Information Administration. (2016). International Energy Outlook 2016. Washington DC: U.S. Energy Information Administration.

U.S. Energy Information Administration. (2017). Short-Term Energy Outlook November 2017.

Uihlein, A., \& Magagna, D. (2016). Wave and Tidal current energy - A review of the current state of researche beyond technology. Renewable and Sustainable Energy Reviews 58, 1070 - 1081.

UOL Confere. (November de 12 de 2017). Fonte: UOL Confere: https://noticias.uol.com.br/confere/ultimas-noticias/2017/03/28/uol-conferetrump-contradiz-estudos-ao-defender-economia-antes-do-clima.htm

Wang, S., Li, Q., Fang, C., \& Zhou, C. (2016, January 15). The relationship between economic growth, energy consumption, and $\mathrm{CO} 2$ emissions: Empirical evidence from China. Science of The Total Environment, Volume 542, pp. 360 $-371$.

Wilcox, D. (1994). Turbulence Modeling for CFD. California: DCW Industries. 
World Energy Council. (2016). World Energy Resources - 2016. London: World Energy Council.

Zhang et al. (2017). Experimental Analysis and Evaluayion of the Numberical Prediction of Wake Characteristics of Tidal Stream Turbine. Energies(10). 


\section{ATTACHMENT A - Potential Fluid Flow}

\section{A.1 - Flow through a circular cylinder}

Using the definition of complex potential from the equation (5.30) it is possible to transform two domains by a transformation known as conformal mapping. One can use a specific transformation called Kutta-Juokowski transformation to transform a cylindrical domain to a plane domain. The transformation is defined by the equation below.

$$
\zeta=\xi+i \eta=z+\frac{C^{2}}{z}
$$

Transforming the flow $\Phi(\xi)=U . \xi$ related to uniform stream to the plane z, according to the Kutta-Jukowisky transformation, where the constant is equal to the cylinder radius, the complex potential becomes:

$$
\Phi(z)=U\left(z+\frac{R^{2}}{z}\right)=U \cdot r\left[e^{i \theta}+\left(\frac{R}{r}\right)^{2} e^{-i \theta}\right]
$$

Considering only the real part of the potential:

$$
\Phi(r, \theta)=U r\left[1+\left(\frac{R}{r}\right)^{2}\right] \cos (\theta)
$$

Transforming the last equation to Cartesian coordinates:

$$
\Phi(x, y)=U x\left(1+\frac{R^{2}}{x^{2}+y^{2}}\right)
$$

Figure 70 - Kutta-Juokowski transformation applied to a circular cylinder

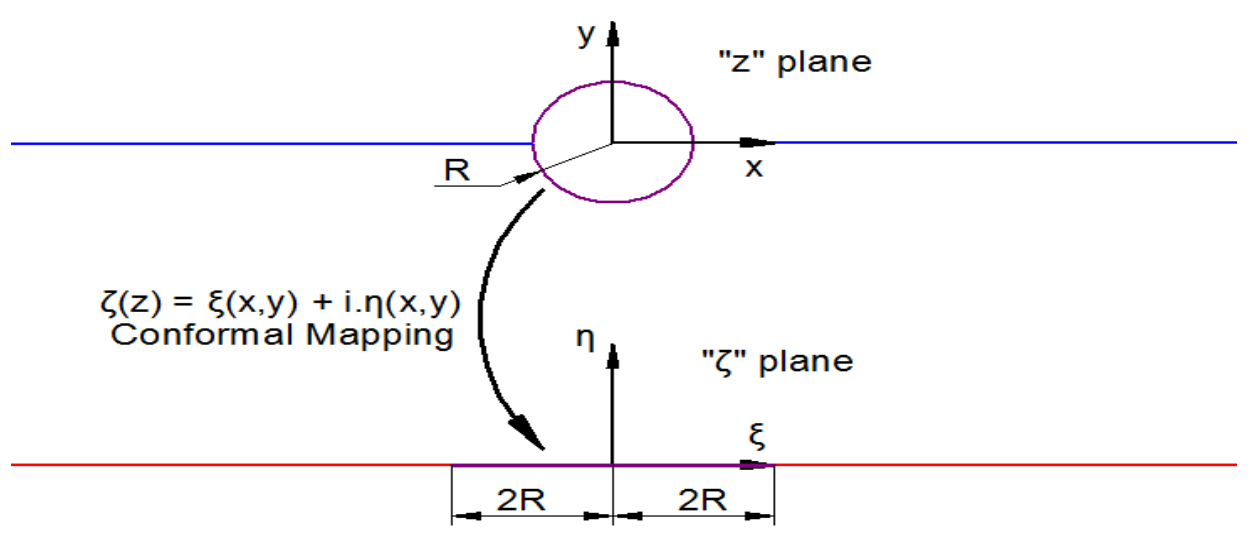




\section{A.2 - Gravity Waves}

The gravity waves for high depths could be analysed using the potential flow theory. For this Phenom, the seabed viscous effects are negligible and due to the oscillation scales, the turbulent effects could be unconsidered. Hence, the fluid flow shall respect the Laplace's equation.

According to (Newman, Marine Hydrodynamics, 1977-A), the boundary conditions are:

i. No flux of mass through the free surface $\eta(x, t)$

ii. Constant pressure along the free surface

iii. No flux of mass through the seabed $\eta(x, t)$

The first boundary condition states that a particle belonging to the free surface must remain always in the free surface. It means that the velocity of the particle must be always tangent to the free surface curve. Defining the curve in a 2D space representing the free surface, $\gamma(x, t)=[x, \eta(x, t)]$. The velocity vector on the free surface is given by:

$$
\frac{d}{d t} \gamma(x, t)=\left[\frac{d}{d t} x, \frac{d}{d t} \eta(x, t)\right]=\left[\frac{d x}{d t}, \frac{\partial \eta}{\partial t}+\frac{\partial \eta}{\partial x} \cdot \frac{d x}{d t}\right]
$$

The velocity vector of a particle on the free surface is given by the gradient of the velocity potential:

$$
\vec{v}(x, z, t)=\left[\frac{\partial \phi_{F S}}{\partial x}, \frac{\partial \phi_{F S}}{\partial z}\right]
$$

Where:

$\phi_{F S} \quad$ Is the potential due to the free surface disturbance

Since both velocity vector and tangent vector to the free surface are parallel, it means that the vectorial product between then is equal to zero. Hence:

$$
\frac{\partial \phi_{F S}}{\partial x} \cdot\left(\frac{\partial \eta}{\partial t}+\frac{\partial \eta}{\partial x} \cdot \frac{d x}{d t}\right)-\frac{\partial \phi_{F S}}{\partial z} \frac{d x}{d t}=0
$$


However, the derivative of $x$ with respect to time is equal the velocity component in $x$ direction. Rearranging the terms, it is given that:

$$
\frac{\partial \phi_{F S}}{\partial x} \cdot\left(\frac{\partial \eta}{\partial t}+\frac{\partial \eta}{\partial x} \cdot \frac{\partial \phi_{F S}}{\partial x}-\frac{\partial \phi_{F S}}{\partial z}\right)=0
$$

The same result is achieved by assuming that the material derivative of the difference $z-\eta$ is zero on the free surface, which is used on (Newman, Marine Hydrodynamics, 1977-A). This equation results in:

$$
\frac{\partial \phi_{F S}}{\partial z}=\frac{\partial \eta}{\partial t}+\frac{\partial \eta}{\partial x} \cdot \frac{\partial \phi_{F S}}{\partial x}
$$

The second boundary condition, so-called Dynamic Boundary condition, states the pressure is constant along the free surface. Calling the Bernoulli equation, assuming the gravity vector is in the $z$ direction, assuming a bi-dimensional case, and passing the pressure term for the right hand of the equation, analysing the free surface, is given that:

$$
\frac{\partial \phi_{F S}}{\partial t}+\frac{1}{2}\left[\left(\frac{\partial \phi_{F S}}{\partial x}\right)^{2}+\left(\frac{\partial \phi_{F S}}{\partial z}\right)^{2}\right]+g \eta=C t e-\frac{p}{\rho}
$$

The free surface could be written in function of atmospheric pressure and velocity potential:

$$
\eta=\frac{1}{g}\left\{C t e-\frac{p}{\rho}-\frac{\partial \phi_{F S}}{\partial t}-\frac{1}{2}\left[\left(\frac{\partial \phi_{F S}}{\partial x}\right)^{2}+\left(\frac{\partial \phi_{F S}}{\partial z}\right)^{2}\right]\right\}
$$

Deriving it with respect to the time, both pressure and the Bernoulli's equation constant vanishes, since both are constant. It gives that:

$$
\frac{\partial \eta}{\partial t}=-\frac{1}{g} \frac{\partial^{2} \phi_{F S}}{\partial t^{2}}-\frac{1}{g}\left[\frac{\partial \phi_{F S}}{\partial x} \overrightarrow{e_{x}}+\frac{\partial \phi_{F S}}{\partial z} \overrightarrow{e_{z}}\right] \cdot\left[\frac{\partial}{\partial x} \overrightarrow{e_{x}}+\frac{\partial}{\partial z} \overrightarrow{e_{z}}\right] \frac{\partial \phi_{F S}}{\partial t}
$$

For the linear wave theory, the non-linear terms are neglected on (A.2.5) and (A.2.7). Substituting the derivative of the free surface with respect to time from equation. (A.2.7) in the equation (A.2.5) is deduced the differential equation for linear gravity wave theory, valid for the free surface. 


$$
\frac{\partial^{2} \phi_{F S}}{\partial t^{2}}+g \frac{\partial \phi_{F S}}{\partial z}=0
$$

Assuming the free surface could be written in terms of a complex Fourier Series:

$$
\eta(x, t)=\sum_{m=1}^{\infty} A_{m} \cdot \exp \left[i\left(\omega_{m} t+k_{m} x\right)\right]
$$

Where:

$\omega_{m} \quad$ Is the angular frequency associated with the m-th component;

$k_{m} \quad$ Is the wave number associated with the m-th component;

$A_{m} \quad$ Is the amplitude associated with the m-th component;

From the linearized equation (A.2.5)

$$
\frac{\partial \phi_{F S}}{\partial z}=\frac{\partial \eta}{\partial t}=\sum_{m=1}^{\infty} i . \omega_{m} \cdot A_{m} \cdot \exp \left[i\left(\omega_{m} t+k_{m} x\right)\right]
$$

Substituting on the differential equation:

$$
\left.\frac{\partial^{2} \phi_{F S}}{\partial t^{2}}\right|_{z=\eta}+g \sum_{m=1}^{\infty} i \cdot \omega_{m} \cdot A_{m} \cdot \exp \left[i\left(\omega_{m} t+k_{m} x\right)\right]=0
$$

Since this equation is linear, there is a particular solution for each component of the Fourier Series:

$$
\left.\frac{\partial^{2} \phi_{F S}}{\partial t^{2}}\right|_{z=\eta}=-i \cdot g \cdot \omega_{m} \cdot A_{m} \cdot \exp \left[i\left(\omega_{m} t+k_{m} x\right)\right]
$$

According to the linearity of this equation with respect to $x, z$ and $t$, the solution could be separated in:

$$
\phi_{F S}^{m}(x, z, t)=\phi_{F S}^{m, t}(t) \cdot \phi_{F S}^{m, x}(x) \cdot \phi_{F S}^{m, z}(z)
$$

Where:
$\phi_{F S}^{m} \quad$ Is the potential of $\mathrm{m}$-th component;
$\phi_{F S}^{m, t} \quad$ Is the potential function of the time of m-th component;
$\phi_{F S}^{m, x} \quad$ Is the potential function of the x-direction of $\mathrm{m}$-th component;
$\phi_{F S}^{m, z} \quad$ Is the potential function of the z-direction of $\mathrm{m}$-th component; 
Substituting (A.2.13) in (A.2.12):

$$
\left.\frac{\partial^{2} \phi_{F S}}{\partial t^{2}}\right|_{z=\eta}=\phi_{F S}^{m, x}(x) \cdot\left[\left.\phi_{F S}^{m, z}(z)\right|_{z=\eta}\right] \frac{d^{2} \phi_{F S}^{m, t}}{d t^{2}}
$$

Hence, the differential equation becomes:

$$
\frac{d^{2} \phi_{F S}^{m, t}}{d t^{2}}=\frac{-i \cdot g \cdot \omega_{m} \cdot A_{m}}{\phi_{F S}^{m, x}(x) \cdot\left[\left.\phi_{F S}^{m, z}(z)\right|_{z=\eta}\right]} \cdot\left[\exp \left(i \omega_{m} t\right)\right]\left[\exp \left(i k_{m} x\right)\right]
$$

Integrating two times with respect to time:

$$
\phi_{F S}^{m, t}(t)=\frac{1}{\phi_{F S}^{m, x}(x) \cdot\left[\left.\phi_{F S}^{m, z}(z)\right|_{z=\eta}\right]} \cdot \frac{i \cdot g \cdot A_{m}}{\omega_{m}} \cdot\left[\exp \left(i \omega_{m} t\right)\right]\left[\exp \left(i k_{m} x\right)\right]
$$

Since the component $\phi_{F S}^{m, t}(t)$ must be independent from $x$, it is given that:

$$
\phi_{F S}^{m, x}(x)=\exp \left(i k_{m} x\right)
$$

Hence, the component $\phi_{F S}^{m, t}(t)$, becomes:

$$
\phi_{F S}^{m, t}(t)=\frac{1}{\left[\left.\phi_{F S}^{m, z}(z)\right|_{z=\eta}\right]} \cdot \frac{i \cdot g \cdot A_{m}}{\omega_{m}} \cdot\left[\exp \left(i \omega_{m} t\right)\right]
$$

From the Laplace's equation, is obtained the component for $z$ direction:

$$
\frac{\partial^{2} \phi_{F S}^{m}}{\partial x^{2}}+\frac{\partial^{2} \phi_{F S}^{m}}{\partial z^{2}}=0 \rightarrow \frac{1}{\phi_{F S}^{m, z}} \frac{d^{2} \phi_{F S}^{m, z}}{d z^{2}}=-\frac{1}{\phi_{F S}^{m, x}} \frac{d^{2} \phi_{F S}^{m, x}}{d x^{2}}
$$

Substituting the results from (A.2.17):

$$
\frac{d^{2} \phi_{F S}^{m, z}}{d z^{2}}-k^{2} \phi_{F S}^{m, z}=0
$$

The equation becomes:

$$
\phi_{F S}^{m, z}(z)=A_{m}^{z} \cosh (k z)+B_{m}^{z} \sinh (k z)
$$


Substituting this on the definition of $\phi_{F S}^{m}(x, z, t)$

$$
\phi_{F S}^{m}(x, z, t)=i \frac{A_{m}^{z} \cosh (k z)+B_{m}^{z} \sinh (k z)}{A_{m}^{z} \cosh (k \eta)+B_{m}^{z} \sinh (k \eta)} \cdot \frac{g \cdot A_{m}}{\omega_{m}} \cdot \exp \left[i\left(\omega_{m} t+k_{m} x\right)\right]
$$

The derivative of this expression in $z$ direction is equal to:

$$
\frac{\partial}{\partial z} \phi_{F S}^{m}(x, z, t)=i k \frac{A_{m}^{z} \sinh (k z)+B_{m}^{z} \cosh (k z)}{A_{m}^{z} \cosh (k \eta)+B_{m}^{z} \sinh (k \eta)} \cdot \frac{g \cdot A_{m}}{\omega_{m}} \cdot \exp \left[i\left(\omega_{m} t+k_{m} x\right)\right]
$$

It is equal to the derivative of the free surface with respect to time, on the free surface:

$$
i \frac{\partial}{\partial z} \phi_{F S}^{m}(x, z, t)=i \cdot \omega_{m} \cdot A_{m} \cdot \exp \left[i\left(\omega_{m} t+k_{m} x\right)\right]
$$

It gives that:

$$
\frac{A_{m}^{z} \sinh (k \eta)+B_{m}^{z} \cosh (k \eta)}{A_{m}^{z} \cosh (k \eta)+B_{m}^{z} \sinh (k \eta)}=\frac{\omega_{m}^{2}}{g \cdot k}
$$

Since on the seabed, the velocity component in $z$ direction is equal to zero, according to the boundary condition iii, it is given that:

$$
\begin{aligned}
\frac{\partial}{\partial z} \phi_{F S}^{m}(x, z, t) & =i k \frac{A_{m}^{z} \sinh \left(-k h_{f}\right)+B_{m}^{z} \cosh \left(-k h_{f}\right)}{A_{m}^{z} \cosh (k \eta)+B_{m}^{z} \sinh (k \eta)} \cdot \frac{g \cdot A_{m}}{\omega_{m}} \cdot \exp \left[i \left(\omega_{m} t\right.\right. \\
& \left.\left.+k_{m} x\right)\right]=0
\end{aligned}
$$

Since it expression is valid independently from $\eta$ :

$$
A_{m}^{Z} \sinh \left(-k h_{f}\right)+B_{m}^{Z} \cosh \left(-k h_{f}\right)=0
$$

Assuming that the hyperbolic sin is an odd function and the hyperbolic cosine is an even function, it gives that:

$$
B_{m}^{z}=A_{m}^{z} \cdot \tanh \left(k h_{f}\right)
$$


The expression (A.2.26) is valid for every surface elevation. Hence, for $\eta$ equals to zero:

$$
\frac{B_{m}^{Z}}{A_{m}^{z}}=\frac{\omega_{m}^{2}}{g \cdot k}
$$

Substituting (A.2.28) in (A.2.29) is deduced the finite depth water dispersion relation:

$$
\omega_{m}^{2}=g \cdot k_{m} \cdot \tanh \left(k_{m} h_{f}\right)
$$

Substituting the expression (A.2.28)on the equation (A.2.25)is found that the constant $A_{m}^{z}$ is equal to $\left[\tanh \left(k h_{f}\right)\right]^{-1}$. By algebra, the expression for $\phi_{F S}^{m}(x, z, t)$ becomes:

$$
\phi_{F S}^{m}(x, z, t)=i \frac{\cosh \left[k_{n}\left(z+h_{f}\right)\right]}{\cosh \left(k_{n} z\right)} \cdot \frac{g \cdot A_{m}}{\omega_{m}} \cdot e^{i\left(\omega_{m} t+k_{m} x\right)}
$$

Composing all terms from the Fourier's series, is given:

$$
\phi_{F S}(x, z, t)=\sum_{m=1}^{\infty} i \frac{\cosh \left[k_{n}\left(z+h_{f}\right)\right]}{\cosh \left(k_{n} z\right)} \cdot \frac{g \cdot A_{m}}{\omega_{m}} \cdot e^{i\left(\omega_{m} t+k_{m} x\right)}
$$

The velocity components are given by the derivative of the potential:

$$
\begin{aligned}
& u_{F S}(x, z, t)=\frac{\partial \phi_{F S}}{\partial x}=\sum_{m=1}^{\infty}-\frac{\cosh \left[k_{n}\left(z+h_{f}\right)\right]}{\cosh \left(k_{n} z\right)} \cdot \frac{g \cdot k_{m} \cdot A_{m}}{\omega_{m}} \cdot e^{i\left(\omega_{m} t+k_{m} x\right)} \\
& w_{F S}(x, z, t)=\frac{\partial \phi_{F S}}{\partial z}=\sum_{m=1}^{\infty} i \frac{\cosh \left[k_{n}\left(z+h_{f}\right)\right]}{\cosh \left(k_{n} z\right)} \cdot \frac{g \cdot k_{m} \cdot A_{m}}{\omega_{m}} \cdot e^{i\left(\omega_{m} t+k_{m} x\right)}
\end{aligned}
$$

Analysing no the "n-th" component of the induced velocities, it is possible to analyse the kinetic energy due to the fluid particles movement. The density of kinetic energy is given by:

$$
\widehat{\mathcal{T}}(x, z, t)=\frac{\rho}{2}\left[\left(u_{F S}^{n}\right)^{2}+\left(w_{F S}^{n}\right)^{2}\right]
$$

Where:

$\hat{\mathcal{T}} \quad$ Is the Kinetic energy density; 
Substituting the results from (A.2.33) and (A.2.34):

$$
\widehat{\mathcal{T}}_{m}(x, z, t)=\frac{\rho}{2} \frac{1+\cosh \left[2 k_{m}\left(z+h_{f}\right)\right]}{1+\cosh \left(2 k_{m} h_{f}\right)} \cdot\left(\frac{g \cdot k_{m} \cdot A_{m}}{\omega_{m}}\right)^{2}
$$

Integrate from the lower boundary to the free surface:

$$
\tilde{\mathcal{T}}_{m}(x, z, t)==\frac{\rho \cdot g \cdot k_{m} \cdot A_{m}^{2}}{\tanh \left(k_{m} h_{f}\right)} \cdot \frac{4 \cdot k_{m} \cdot\left(\eta+h_{f}\right)+\sinh \left[2 k_{m}\left(\eta+h_{f}\right)\right]-1}{8 \cdot k_{m} \cdot\left(1+\cosh \left(2 k_{m} h_{f}\right)\right)}
$$

If the depth is considered infinite, the kinetic energy becomes:

$$
\tilde{\mathcal{T}}_{m}(x, z, t)=\frac{\rho \cdot g \cdot A_{m}^{2}}{4} \cdot \exp \left(2 \cdot k_{m} \cdot \eta\right)
$$

For the gravitational potential energy, the term related to gravity in the Bernoulli's Equation is integrated from the seabed to the surface:

$$
\tilde{\mathcal{V}}_{m}(x, z, t)=\int_{-h_{f}}^{\eta} \rho \cdot g \cdot z \cdot d z=\frac{1}{2} \cdot \rho \cdot g \cdot \eta^{2}-\frac{1}{2} \cdot \rho \cdot g \cdot h_{f}^{2}
$$

Where:

$\tilde{v}_{m} \quad$ Is the mean potential energy density;

Since the restoring force is given by the first derivative of the potential energy, the constant term could be neglected. Therefore:

$$
\tilde{\mathcal{V}}_{m}(x, z, t)=\frac{1}{2} \cdot \rho \cdot g \cdot \eta^{2}
$$

The total energy density per unit of area is given by the sum between equations (A2.38) and (A.2.40). 


\section{ATTACHMENT B $-\boldsymbol{k}-\boldsymbol{\omega}-\mathrm{SST}$ Model}

The model, as the Wilcox $k-\omega$ calculates the turbulent viscosity from the turbulent kinetic energy and the rotational turbulent kinetic energy. Therefore, the $k$ and $\omega$ transport equations are solved. These two equations are presented below.

$$
\begin{aligned}
\frac{\partial}{\partial \mathrm{t}}(\rho k)+ & \frac{\partial}{\partial x_{j}}\left(\rho \overline{u_{j}} k\right)=\frac{\partial}{\partial x_{j}}\left[\left(\mu+\frac{\mu_{t}}{\sigma_{k 3}}\right) \frac{\partial k}{\partial x_{j}}\right]+\mathcal{P}_{k}-\beta^{\prime} \rho k \omega+\mathcal{P}_{k b} \\
\frac{\partial}{\partial \mathrm{t}}(\rho \omega)+\frac{\partial}{\partial x_{j}} & \left(\rho \bar{u}_{j} \omega\right) \\
& =\frac{\partial}{\partial x_{j}}\left[\left(\mu+\frac{\mu_{t}}{\sigma_{\omega 3}}\right) \frac{\partial \omega}{\partial x_{j}}\right]+\left(1-F_{1}\right) 2 \rho \frac{1}{\sigma_{\omega 2} \omega} \cdot \frac{\partial k}{\partial x_{j}} \frac{\partial \omega}{\partial x_{j}}+\alpha_{3} \frac{\omega}{k} \mathcal{P}_{k} \\
& -\beta_{3} \rho \omega^{2}+\mathcal{P}_{\omega b}
\end{aligned}
$$

The turbulent viscosity is calculated by:

$$
\mu_{t}=\rho \frac{a_{1} k}{\max \left(a_{1} \omega, S F_{2}\right)}
$$

The coefficient $\mathrm{F}_{1}$ is calculated thorugh the following procedure:

$$
\begin{gathered}
C D_{K W}=\max \left(2 \rho \frac{1}{\sigma_{\omega 2} \omega} \cdot \frac{\partial k}{\partial x_{j}} \frac{\partial \omega}{\partial x_{j}}, 10^{-10}\right) \\
\arg _{1}=\min \left[\max \left(\frac{\sqrt{k}}{\beta^{\prime} \omega y}, \frac{500 v}{y^{2} \omega}\right), \frac{4 \rho k}{C D_{K W} \sigma_{\omega 2} y^{2}}\right] \\
\mathrm{F}_{1}=\tanh \left(\arg _{1}^{4}\right)
\end{gathered}
$$

And the coefficient $\mathrm{F}_{2}$ is calculated thorugh the following procedure:

$$
\begin{gathered}
\arg _{2}=\max \left(\frac{2 \sqrt{k}}{\beta^{\prime} \omega y}, \frac{500 v}{y^{2} \omega}\right) \\
\mathrm{F}_{2}=\tanh \left(\arg _{2}^{2}\right)
\end{gathered}
$$


The coefficients $\sigma_{k 3}$ and $\sigma_{\omega 3}$ are calculate through the eqs (B.9) and (B.10) and the other coefficients are shown in the Table 7.

$$
\begin{gathered}
\sigma_{k 3}=\sigma_{k 1} F_{1}+\left(1-F_{1}\right) \sigma_{k 2} \\
\sigma_{\omega 3}=\sigma_{\omega 1} F_{1}+\left(1-F_{1}\right) \sigma_{\omega 2}
\end{gathered}
$$

Table 7 - Coefficients for $\mathbf{k}-\boldsymbol{\omega}$ - SST

\begin{tabular}{|c|c|}
\hline Coefficient & Value \\
\hline$\beta^{\prime}$ & 0.09 \\
\hline$\alpha_{1}$ & 0.556 \\
\hline$\beta_{1}$ & 0.075 \\
\hline$\alpha_{1}$ & 2 \\
\hline$\sigma_{\omega 1}$ & 2 \\
\hline$\alpha_{2}$ & 0.44 \\
\hline$\beta_{2}$ & 0.828 \\
\hline$\sigma_{k 2}$ & 1 \\
\hline$\sigma_{\omega 2}$ & 1.168 \\
\hline
\end{tabular}




\section{ATTACHMENT C - Wall Functions}

The ANSYS CFX® uses scalable wall functions to model the boundary layer flow for meshes which presents high $y^{+}$values in the wall. It is necessary to reduce the computational cost for analysis which not requires the full boundary layer resolution such the solution of problems that involves only the calculation of the integral hydrodynamic loads in bodies.

The ASYS® users guide (ANSYS Inc., 2016, p. Section 2.8.1) concerns about the usage of wall functions in CFD problems. Some effects depend on an accurate prediction of phenoms such as boundary layer separation or the boundary layer velocity profile.

However, for simpler problems, this methodology allows to achieve reasonable results even with coarser meshes which leads to a computational time decrease or the inclusion of more complex geometries and boundary conditions.

The code uses the formulation from (Launder \& Spalding, 1974) in which the near wall tangential velocity is related to the wall-shear-stress by means of a logarithmic relation.

$$
u^{+}=\frac{1}{\kappa} \ln \left(y^{+}\right)+C
$$

Where:

$k \quad$ Is a constant;

C Is a constant.

The dimensionless distance from the wall, $y^{+}$, is extimated by:

$$
y^{+}=\frac{\Delta y \cdot u_{\tau}}{v}
$$

Near to the separation point, the wall velocity approaches to zero. For the logarithmic region, an alternative velocity scale, $u^{*}$, could be applied:

$$
u^{*}=C_{\mu}^{(1 / 4)} k^{(1 / 2)}
$$


This alternative definition does not go to zero when the velocity goes to zero. Based on this definition, the following explicit equation for the velocity scale can be obtained:

$$
u_{\tau}=\frac{U}{\frac{1}{\kappa} \ln \left(y^{+}\right)+C}
$$

And hence, the wall shear stress is calculated through the following equation:

$$
\tau_{w}=\rho u^{*} u_{\tau}
$$

And finally, the alternative length scale is given by:

$$
y^{*}=\frac{\Delta y \cdot u^{*}}{v}
$$

The turbulent kinetic energy dissipation is calculated by the following equation:

$$
\varepsilon=\frac{\Delta y \cdot u^{*}}{v \cdot \max \left(11.06 ; y^{*}\right)} \frac{C_{\mu}^{(3 / 4)} k^{(3 / 2)}}{\kappa}
$$

The adoption of $\max \left(11.06 ; y^{*}\right)$ instead of only the value of $y^{*}$ is due to issues related with mesh refinement near to the wall. Even with those approaches, in the ANSYS $\AA^{\circ}$ users guide, the following points are recommended:

- To fully resolve the boundary layer, you should put at least 10 nodes into the boundary layer.

- The upper limit for $y^{+}$is a function of the device Reynolds number. For example, a large ship may have a Reynolds number $10^{9}$ and $y^{+}$can safely go to values much greater than 1000 . For lower Reynolds numbers (for example, a small pump), the entire boundary layer might only extend to around $y^{+}=300$. In this case, a fine near wall spacing is required to ensure a sufficient number of nodes in the boundary layer. 


\section{ATTACHMENT D - Actuator Disk Theory}

\section{Open Flow Model (Betz Limit)}

The domain is divided in turbine region that analyses the flow that passes through the turbine disk and the by-pass region that analyses the flow that passes around the turbine disk. The analysed sections are the domain inlet, domain outlet, immediately before turbine region and immediately after turbine region. The superscripts used for the analysis are:

Table 8 - Open flow model analysed sections

\begin{tabular}{|l|c|c|c|c|}
\hline \multirow{2}{*}{ Section } & \multicolumn{2}{|c|}{ Turbine Region } & \multicolumn{2}{c|}{ By-Pass Region } \\
\cline { 2 - 5 } & Subscript & Velocity & Subscript & Velocity \\
\hline Inlet & $i, t$ & $u_{i, t}=u$ & $i, b$ & - \\
\hline Turbine Upstream & $u t, t$ & $u_{u t, t}=\alpha_{t} u$ & $u t, b$ & - \\
\hline Turbine Downstream & $d t, t$ & $u_{d t, t}=\alpha_{t} u$ & $d t, b$ & - \\
\hline Outlet & $o, t$ & $u_{o, t}=\alpha_{o} u$ & $o, b$ & $u_{o, b}=u$ \\
\hline
\end{tabular}

From the balance of mass, is possible to relate the ratio between the inlet and outlet areas with the turbine disk area following the ratio between its velocities. Rearranging the equation of continuity:

$$
A_{o}=\frac{\overline{\bar{u}}_{i}}{\overline{\bar{u}}_{o}} A_{i}
$$

Applying this relation between the turbine upstream section and inlet and then between the outlet and inlet turbine regions are deduced:

- Turbine Inlet Area:

$$
A_{i, t}=\frac{u_{u t, t}}{u_{i, t}} A_{u t, t}=\alpha_{t} A
$$

- Turbine Outlet Area:

$$
A_{o, t}=\frac{u_{i, t}}{u_{o, t}} A_{i, t}=\frac{\alpha_{t}}{\alpha_{o}} A
$$


Figure 71 - Scheme for open flow through an axial turbine

\section{Inlet "i"}

\section{Outlet "o"}

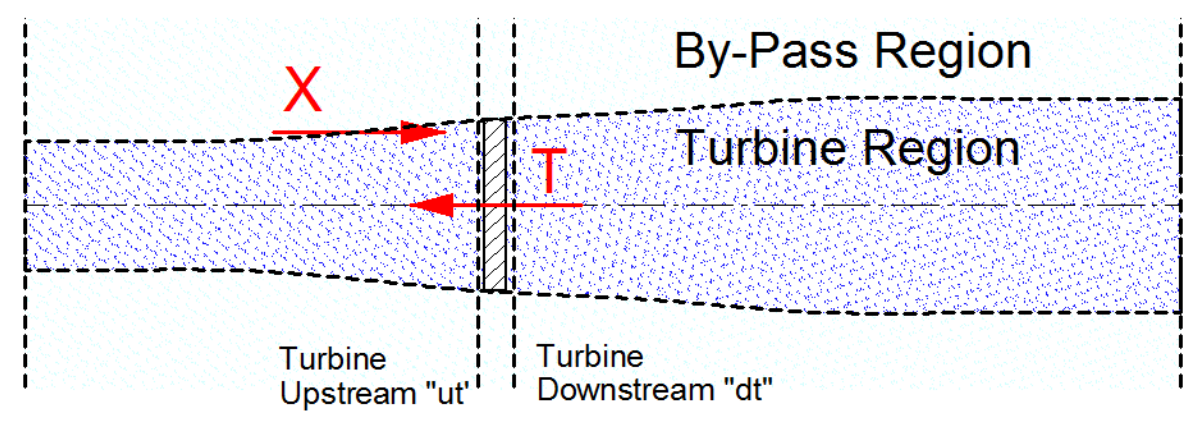

The energy balance from Bernoulli equation between turbine upstream and turbine downstream relates its pressure following:

$$
\frac{u_{u t, t}^{2}}{2}+\frac{p_{u t, t}}{\rho}=\frac{u_{d t, t}^{2}}{2}+\frac{p_{d t, t}}{\rho}+\frac{T}{\rho \cdot A}
$$

However, according to the continuity equation, the upstream mean velocity must be equal to the downstream mean velocity. The, the difference between the upstream and the downstream pressures is due to the thrust generated by the turbine disk.

$$
p_{u t, t}-p_{d t, t}=\frac{T}{A}
$$

Analysing now two streamlines, one between the inlet section and upstream section and other between the downstream section and the outlet section. It is seen that there are no forces acting in both streamlines. Then, the Bernoulli's equation without losses could be used to obtain the upstream pressure in function of inlet pressure and the downstream pressure in function of the downstream pressure.

$$
\begin{gathered}
\frac{u_{u t, t}^{2}}{2}+\frac{p_{u t, t}}{\rho}=\frac{u_{i, t}^{2}}{2}+\frac{p_{i, t}}{\rho} \rightarrow \frac{p_{i, t}}{\rho}=\frac{p_{u t, t}}{\rho}-\frac{\left(u_{i, t}^{2}-u_{u t, t}^{2}\right)}{2}=\frac{p_{u t, t}}{\rho}-\frac{\left(1-\alpha_{t}^{2}\right)}{2} u^{2} \\
\frac{u_{d t, t}^{2}}{2}+\frac{p_{d t, t}}{\rho}=\frac{u_{o, t}^{2}}{2}+\frac{p_{o, t}}{\rho} \rightarrow \frac{p_{0, t}}{\rho}=\frac{p_{d t, t}}{\rho}+\frac{\left(u_{o, t}^{2}-u_{d t, t}^{2}\right)}{2}=\frac{p_{d t, t}}{\rho}+\frac{\left(\alpha_{t}^{2}-\alpha_{o}^{2}\right)}{2} u^{2}
\end{gathered}
$$


Then, the pressure difference between inlet and outlet is given by:

$$
p_{i, t}-p_{0, t}=p_{u t, t}-p_{d t, t}+\frac{\left(1-\alpha_{0}^{2}\right)}{2} \rho u^{2}=\frac{T}{A}-\frac{\left(1-\alpha_{0}^{2}\right)}{2} \rho u^{2}
$$

Since there is no pressure difference between inlet and outlet sections, assuming that the domain is infinite and then the influence of a turbine disk on the domain main pressure is negligible:

$$
\frac{T}{A}=\frac{\left(1-\alpha_{0}^{2}\right)}{2} \rho u^{2}
$$

Applying now the momentum conservation equation in the turbine region between the inlet and the outlet, assuming that there is no pressure difference between these two stations:

$$
u_{o, t}^{2} A_{o, t}-u_{i, t}^{2} A_{i, t}=\frac{X-T}{\rho}
$$

By assumption, the net momentum difference between turbine region and by pass is equal to zero. These assumption is not true, however for the infinite domain consideration is a reasonable approach, there is deduced that:

$$
u^{2} \alpha_{o} \alpha_{t} A-u^{2} \alpha_{t} A=A u^{2} \alpha_{t}\left(\alpha_{o}-1\right)=-\frac{T}{\rho}
$$

Comparing the equations (41) and (39) is found the relation:

$$
\alpha_{t}=\frac{1+\alpha_{o}}{2}
$$

The power absorbed by the turbine is given by the product between the thrust and the mean velocity in the turbine region. Therefore:

$$
P=u_{u t, t} T=\frac{1}{2} \cdot \frac{\left(1+\alpha_{o}\right)\left(1-\alpha_{0}^{2}\right)}{2} \rho A u^{3}=\frac{1}{2} \cdot C_{P} \cdot \rho A u^{3}
$$

It maximum value is found when $\alpha_{o}=1 / 3, \alpha_{t}=2 / 3$ and it is $C_{P}=16 / 27$. It is known as Betz limit (Betz, 1920).

The results of the equation (C.13) are illustrated in the Figure 72 . The maximum $C_{P}$ of $16 / 27$ means that in an optimum design, the maximum power extracted from the free flow is about $59 \%$ of the total energy available in the fluid. 
Figure 72 - Power and thrust coefficients for open flow through an axial turbine

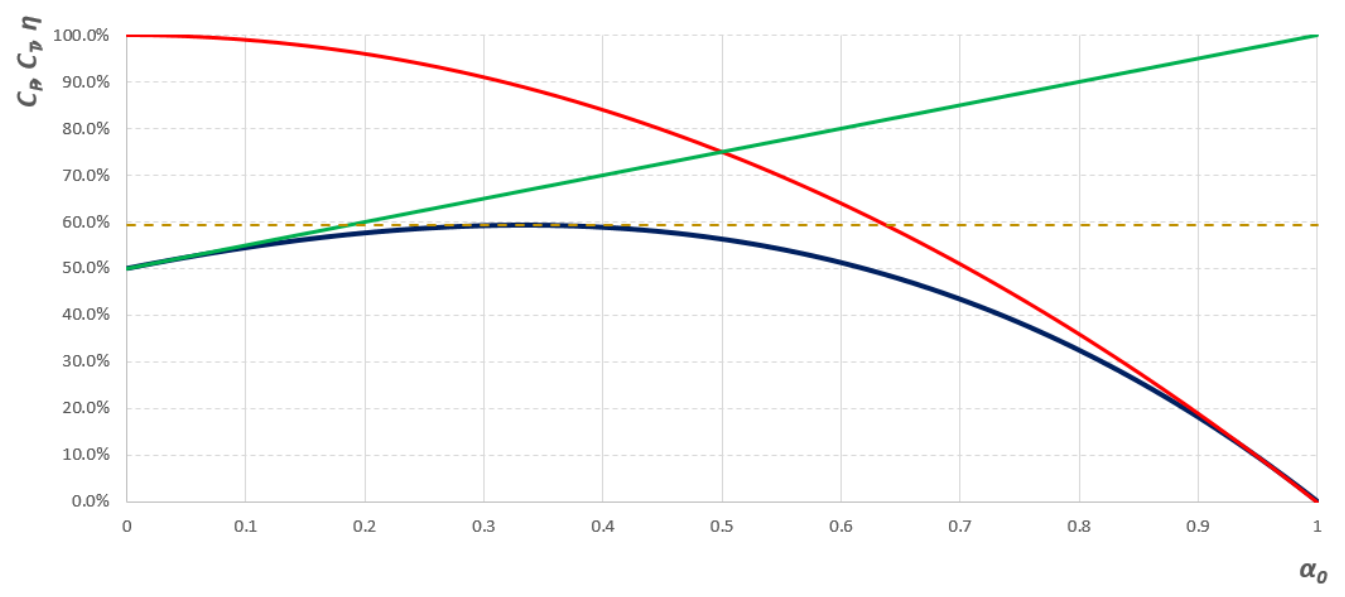

\section{Confined Flow Model (Ducted Flow)}

The main feature of these approach is the introduction of blockage effects in hydrodynamic performance. The equivalence of pressure in inlet and outlet is no more a valid hypothesis and now an additional section, after turbine and by-pass region mixture, is introduced. The flow in the by-pass region is known by losses (Souza, 2011), therefore, the flow confinement is expected to increase the absorbed power by the turbine. Kaplan and Bulb turbines, such as La Rance tidal power farm, works at this principle and it is a good step before considering the free surface as confinement. A remarkable difference between this model and the original one from Betz, is related to the conservation of the quantities in the by-pass region which is not more considered infinite and the introduction of the mixing between both turbine and by-pass flow assuming that in outlet of the domain, the flow velocity profile has been recovered.

Table 9 - Open flow model analysed sections

\begin{tabular}{|l|c|c|c|c|}
\hline \multirow{2}{*}{ Section } & \multicolumn{2}{|c|}{ Turbine Region } & \multicolumn{2}{c|}{ By-Pass Region } \\
\cline { 2 - 5 } & Subscript & Velocity & Subscript & Velocity \\
\hline Inlet & $i, t$ & $u_{i, t}=u$ & $i, b$ & $u_{i, b}=u-$ \\
\hline Turbine Upstream & $u t, t$ & $u_{u t, t}=\alpha_{t} u$ & $u t, b$ & $u_{u t, b}=\frac{1-B \alpha_{T}}{\alpha_{T}-B \alpha_{T}} \cdot u$ \\
\hline Turbine Downstream & $d t, t$ & $u_{d t, t}=\alpha_{t} u$ & $d t, b$ & $u_{d t, b}=\frac{1-B \alpha_{T}}{\alpha_{T}-B \alpha_{T}} \cdot u$ \\
\hline Outlet before mixing & $o b, t$ & $u_{o b, t}=\alpha_{o b} u$ & $o b, b$ & $u_{d t, b}=\frac{1-B \alpha_{T}}{\alpha_{o b}-B \alpha_{T}} \cdot u$ \\
\hline Outlet & $o, t$ & $u_{o, t}=u$ & $o, b$ & $u_{o, b}=u$ \\
\hline
\end{tabular}


Figure 73 - Scheme for a confined flow through an axial turbine

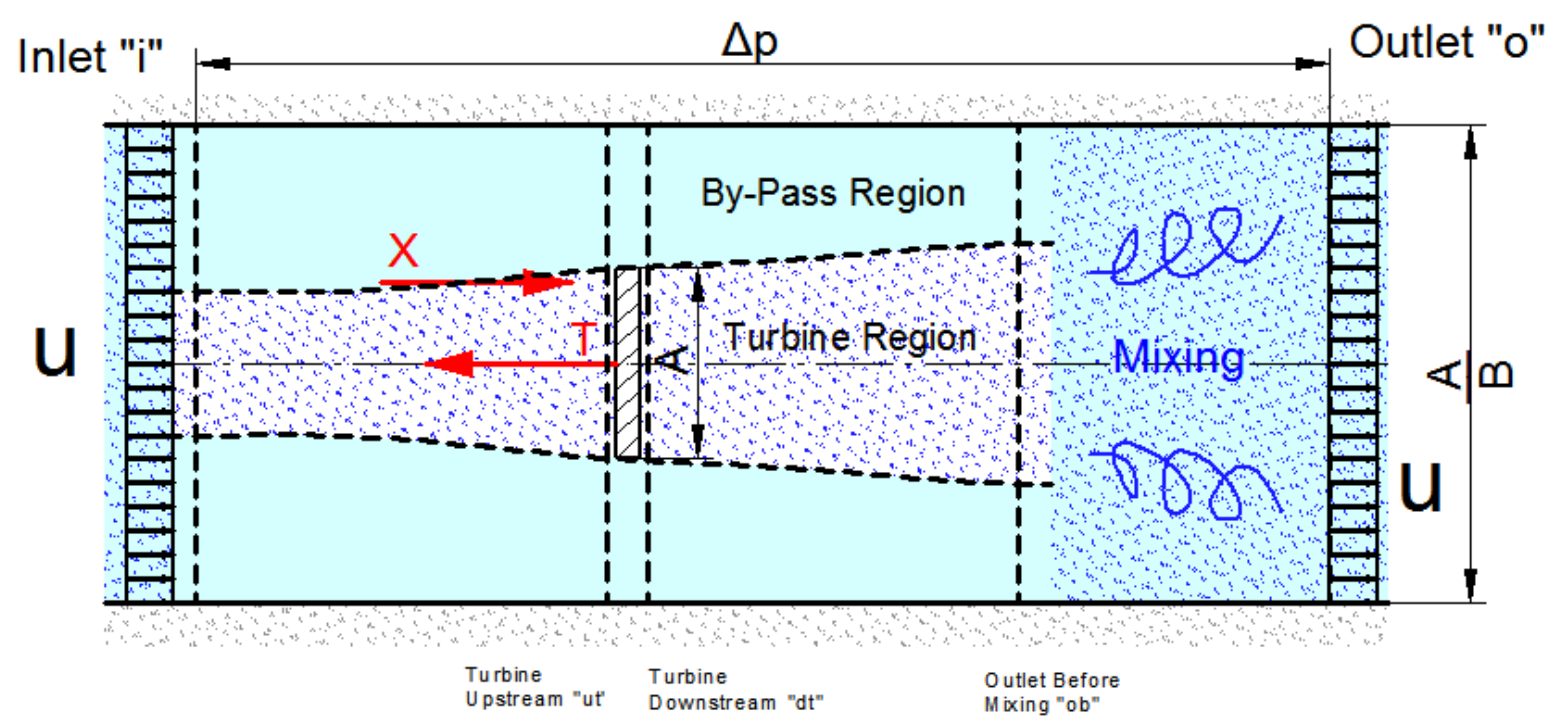

Is defined the blockage ratio $B$ as the ratio between the turbine disk area and the channel area. The velocities in by-pass region are written in function of the blockage ratio in order to maintain the mass bulk flow constant through all analysed sections. By this assumption and calling the mass conservation equation between the inlet and any section " $s$ " is found the relation shown in the equation below. The results for the analysed sections are presented in Table 10.

$$
\alpha_{s, b}=\alpha_{s, t} \frac{1-B \cdot \alpha_{t}}{\alpha_{s, t}-B \cdot \alpha_{t}}
$$

The Bernoulli equation in the by-pass flow, between inlet and outlet before mixing is given by:

$$
\frac{p_{i}}{\rho}+\frac{u^{2}}{2}=\frac{p_{o b}}{\rho}+\frac{\alpha_{o b, b}^{2} \cdot u^{2}}{2}
$$

The Bernoulli equation in the turbine flow, between the station immediately after turbine and the outlet station before mixing is given by:

$$
\frac{p_{a t, t}}{\rho}+\frac{\alpha_{t}^{2} \cdot u^{2}}{2}=\frac{p_{o b}}{\rho}+\frac{\alpha_{o b, t}^{2} \cdot u^{2}}{2}
$$


Table 10 - Confined flow model analysed sections

\begin{tabular}{|l|c|c|c|c|}
\hline \multirow{2}{*}{ Section } & \multicolumn{2}{|c|}{ Turbine Region } & \multicolumn{2}{c|}{ By-Pass Region } \\
\cline { 2 - 5 } & Subscript & Velocity & Subscript & Velocity \\
\hline Inlet & $i, t$ & $u_{i, t}=u$ & $i, b$ & $u_{i, b}=u-$ \\
\hline $\begin{array}{l}\text { Turbine } \\
\text { Upstream }\end{array}$ & $u t, t$ & $u_{u t, t}=\alpha_{t} u$ & $u t, b$ & $u_{u t, b}=\frac{1-B \alpha_{T}}{\alpha_{T}-B \alpha_{T}} \cdot u$ \\
\hline $\begin{array}{l}\text { Turbine } \\
\text { Downstream }\end{array}$ & $d t, t$ & $u_{d t, t}=\alpha_{t} u$ & $d t, b$ & $u_{d t, b}=\frac{1-B \alpha_{T}}{\alpha_{T}-B \alpha_{T}} \cdot u$ \\
\hline $\begin{array}{l}\text { Outlet before } \\
\text { mixing }\end{array}$ & $o b, t$ & $u_{o b, t}=\alpha_{o b} u$ & $o b, b$ & $u_{d t, b}=\frac{1-B \alpha_{T}}{\alpha_{o b}-B \alpha_{T}} \cdot u$ \\
\hline Outlet & $o, t$ & $u_{o, t}=u$ & $o, b$ & $u_{o, b}=u$ \\
\hline
\end{tabular}

Applying Bernoulli equation between inlet and the section immediately before the turbine, in the turbine flow is given that:

$$
\frac{p_{i}}{\rho}+\frac{u^{2}}{2}=\frac{p_{d t, t}}{\rho}+\frac{\alpha_{t}^{2} \cdot u^{2}}{2}
$$

Considering that the pressure drop between the sections immediately upstream and downstream of the turbine is related to the thrust due to the turbine, according to the equation (C.5), combining the equations(C.14), (C.15), (C.16) and (C.17), is given that:

$$
\frac{u^{2}}{2} \alpha_{o b, t}^{2}\left[\left(\frac{1-B \cdot \alpha_{t}}{\alpha_{o b, t}-B \cdot \alpha_{t}}\right)^{2}-1\right]=\frac{T}{\rho A}
$$

Applying the momentum conservation equation between inlet section and outlet before mixing section, is given that:

$$
\frac{\rho u^{2}}{B}\left\{1-\alpha_{o b, t}\left[B \cdot \alpha_{t}+\frac{\left(1-B \cdot \alpha_{t}\right)^{2}}{\alpha_{o b, t}-B \cdot \alpha_{t}}\right]\right\}=-\frac{\left(p_{i}-p_{o b}\right)}{\rho \cdot B}+\frac{T}{\rho A}
$$

Combining (C.19) and (C.20), is given the following quadratic equation:

$$
\alpha_{t}^{2}+\frac{2(1+B) \alpha_{o b, t}^{2}}{B \cdot\left(1-3 \alpha_{o b, t}\right)} \alpha_{t}-\frac{\alpha_{o b, t}^{2}\left(1+\alpha_{o b, t}\right)}{B \cdot\left(1-3 \alpha_{o b, t}\right)}=0
$$

Solving for $\alpha_{t}$, is given that:

$$
\alpha_{t}=\frac{\left(1+\alpha_{o b, t}\right) \alpha_{o b, t}}{(1+B) \alpha_{o b, t}+\sqrt{(1-B)^{2} \alpha_{o b, t}^{2}+B\left(\alpha_{o b, t}-1\right)^{2}}}
$$


Substituting this result on (C.18) is obtained the thrust on the turbine. With the thrust, multiplying by the velocity trough the turbine is obtained the total power extracted from the turbine. For the power lost, is necessary to analyse the mixing process between the stations of outlet before mixing and outlet. This detail is found on (Houlsby et al., 2007). The thrust and power coefficients obtained from this model are given by:

$$
\begin{gathered}
C_{T}=\alpha_{o b, t}^{2}\left[\left(\frac{1-B \cdot \alpha_{t}}{\alpha_{o b, t}-B \cdot \alpha_{t}}\right)^{2}-1\right] \\
C_{P}=\alpha_{t} C_{T}
\end{gathered}
$$

\section{Single Turbine Placed on Open Channel Flow}

In the Figure 74 are shown the sections analysed for the open channel flow through an axial turbine. Appling the Bernoulli Equation between inlet section and outlet section before mixing, considering the assumption of the section "ob" have the same head as the outlet section:

$$
\frac{\mathrm{u}^{2}}{2}+\frac{\mathrm{p}_{\mathrm{atm}}}{\rho}+\mathrm{gh}=\frac{\alpha_{\mathrm{ob}, \mathrm{b}}^{2} \mathrm{u}^{2}}{2}+\frac{\mathrm{p}_{\mathrm{atm}}}{\rho}+\mathrm{g}(\mathrm{h}-\Delta \mathrm{h}) \rightarrow \Delta \mathrm{h}=\frac{\mathrm{u}^{2}}{2 \cdot \mathrm{g}}\left(\alpha_{\mathrm{ob}, \mathrm{b}}^{2}-1\right)
$$

The Table 11 presents the velocities and areas used in the analysis. The process for obtaining these areas comes from the continuity equation. According to (Peiró et al., 2007), the sections "ob" and " $o$ " have the same height difference, and this make sense assuming that the head loss between these two sections is negligible if compared with the head loss due to the turbine. Therefore the area of the section of outlet before mixing, which is given by the sum of turbine and by-pass areas is equal to the area of outlet section. It is represented by the following equation:

$$
A_{o b, b}+A_{o b, t}=A_{o} \rightarrow \frac{\alpha_{t}}{\alpha_{o b, t}} A+\frac{A}{\alpha_{o b, b}}\left(\frac{1-B \alpha_{t}}{B}\right)=\frac{A}{B} \frac{(h-\Delta h)}{h}
$$

Rearranging this equation, is possible to write the velocity at turbine wake, from the stream tube region in outlet before mixing, in function of turbine height difference, blockage ratio and velocity coefficient at turbine. Since

$$
\alpha_{t}=\frac{\alpha_{o b, t}}{B\left(\alpha_{o b, b}-\alpha_{o b, t}\right)}\left[\left(1-\frac{\Delta h}{h}\right) \alpha_{o b, b}-1\right]
$$


From Bernoulli Equation between inlet section and outlet before mixing by pass section, by assumption of same height of outlet section:

$$
\frac{u^{2}}{2}+\frac{p_{a t m}}{\rho}+g h=\frac{\alpha_{o b, b}^{2} u^{2}}{2}+\frac{p_{a t m}}{\rho}+g(h-\Delta h) \rightarrow \Delta h=\frac{u^{2}}{2 \cdot g}\left(\alpha_{o b, b}^{2}-1\right)
$$

From the Bernoulli equation, with a streamline passing through the turbine between the inlet and outlet before mixing, is derived the following expression for turbine force:

$$
F=\rho \cdot A \cdot\left[g \cdot \Delta h+\frac{U^{2}}{2}\left(1-\alpha_{o b, t}^{2}\right)\right]
$$

Another expression for force is found by applying the momentum conservation from the inlet to outlet. Applying it between the inlet section and outlet before mixing section, passing through the free surface:

$$
F=\rho \cdot A \cdot\left\{\frac{g \cdot h}{2 \cdot B} \cdot\left[2 \cdot \frac{\Delta h}{h}-\left(\frac{\Delta h}{h}\right)^{2}\right]+\frac{U^{2}}{2 \cdot B}\left[\frac{\left(1-\alpha_{o b, b}^{2}\right)}{B}+2 \cdot B \cdot \alpha_{t}\left(\alpha_{o b, b}-\alpha_{o b, t}\right)\right]\right\}
$$

By the comparison between (C.27) and (C.28), substituting the results of (C.25) and (C.26) it is derived the following equation for the velocity coefficient for by-pass in outlet before mixing section:

$$
\begin{aligned}
\left(F r_{h}^{2} / 2\right) \alpha_{o b, b}^{4} & +\left(2 \cdot \alpha_{o b, t} \cdot F r_{h}^{2}\right) \alpha_{o b, b}^{3}+\left(2 \cdot B-2-F r_{h}^{2}\right) \alpha_{o b, b}^{2} \\
& +\left(4-4 \alpha_{o b, t}-2 \cdot \alpha_{o b, t} \cdot F r_{h}^{2}\right) \alpha_{o b, b} \\
& +\left(c_{0}=4 \alpha_{o b, t}-2+F r_{h}^{2} / 2-2 \cdot B \cdot \alpha_{o b, t}^{2}\right)=0
\end{aligned}
$$

Figure 74 - Scheme for an open channel flow through an axial turbine

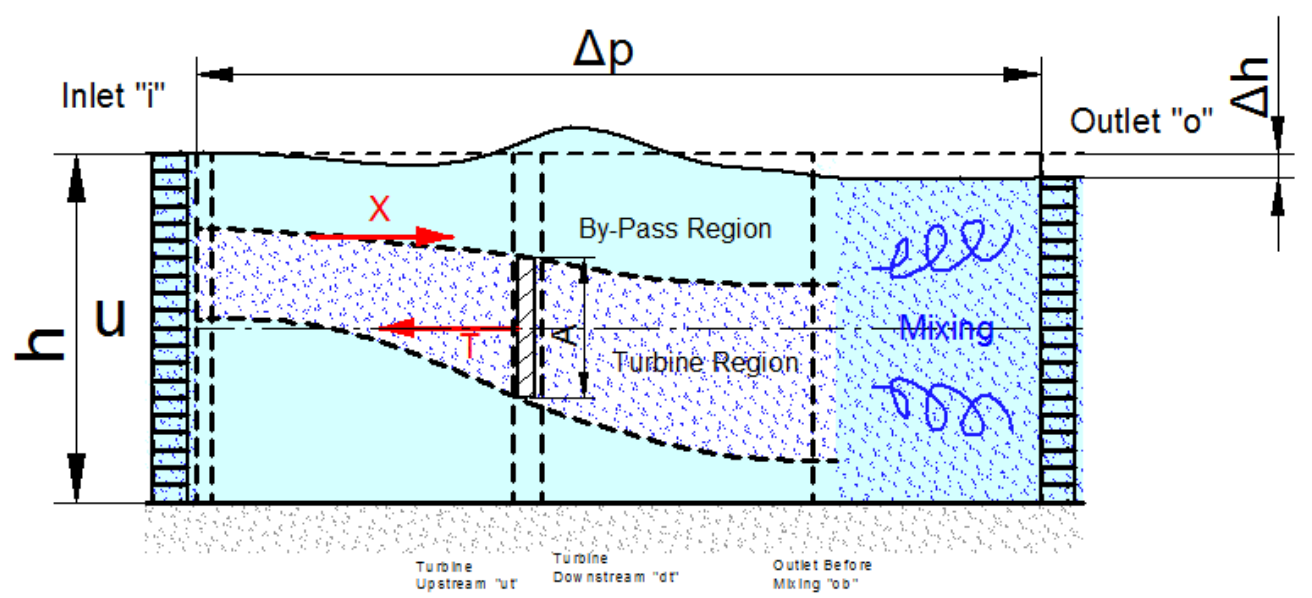


Table 11 - Open channel analysed sections - Velocities and Areas

\begin{tabular}{|c|c|c|c|c|}
\hline \multirow{2}{*}{ Section } & \multicolumn{2}{|c|}{ Turbine Region } & \multicolumn{2}{|c|}{ By-Pass Region } \\
\hline & Subscript & Velocity & Subscript & Velocity \\
\hline Inlet & $i, t$ & $u_{i, t}=u$ & $i, b$ & $u_{i, b}=u-$ \\
\hline Turbine Upstream & $u t, t$ & $u_{u t, t}=\alpha_{t} u$ & $u t, b$ & $u_{u t, b}=\frac{1-B \alpha_{T}}{\alpha_{T}-B \alpha_{T}} \cdot u$ \\
\hline Turbine Downstream & $d t, t$ & $u_{d t, t}=\alpha_{t} u$ & $d t, b$ & $u_{d t, b}=\frac{1-B \alpha_{T}}{\alpha_{T}-B \alpha_{T}} \cdot u$ \\
\hline Outlet before mixing & $o b, t$ & $u_{o b, t}=\alpha_{o b} u$ & $o b, b$ & $u_{d t, b}=\frac{1-B \alpha_{T}}{\alpha_{o b}-B \alpha_{T}} \cdot u$ \\
\hline Outlet & $o, t$ & $u_{o, t}=u$ & $o, b$ & $u_{o, b}=u$ \\
\hline \multirow{2}{*}{ Section } & \multicolumn{2}{|c|}{ Turbine Region } & \multicolumn{2}{|c|}{ By-Pass Region } \\
\hline & \multicolumn{2}{|c|}{ Area } & \multicolumn{2}{|c|}{ Area } \\
\hline Inlet & \multicolumn{2}{|c|}{$A_{i, t}=\alpha_{t} A$} & \multicolumn{2}{|c|}{$A_{i, b}=A\left(\frac{1-B \alpha_{t}}{B}\right)$} \\
\hline Turbine Upstream & \multicolumn{2}{|c|}{$A$} & \multicolumn{2}{|c|}{$A_{u t, b}=\frac{A}{\alpha_{u t, b}}\left(\frac{1-B \alpha_{t}}{B}\right)$} \\
\hline Turbine Downstream & \multicolumn{2}{|c|}{$A$} & \multicolumn{2}{|c|}{$A_{u t, b}=\frac{A}{\alpha_{d t, b}}\left(\frac{1-B \alpha_{t}}{B}\right)$} \\
\hline Outlet before mixing & \multicolumn{2}{|c|}{$A_{o b, t}=\frac{\alpha_{t}}{\alpha_{o b, t}} A$} & \multicolumn{2}{|c|}{$A_{o b, b}=\frac{A}{\alpha_{o b, b}}\left(\frac{1-B \alpha_{t}}{B}\right)$} \\
\hline Outlet & \multicolumn{4}{|c|}{$A_{o}=\frac{A}{B} \frac{(h-\Delta h)}{h}=\frac{A}{\alpha_{o, b} B}$} \\
\hline
\end{tabular}

From the results from equation (C.29) is possible to calculate the height difference from the equation (C.26). With both height difference and the velocity coefficient, is possible to calculate the velocity coefficient in the turbine section. With the equation. (C.27) is possible to determinate the thrust. Hence, the power is determined by the same way that used for open flow model:

$$
P=u_{u t, t} T=\frac{1}{2} \cdot \frac{\left(1+\alpha_{o}\right)\left(1-\alpha_{0}^{2}\right)}{2} \rho A u^{3}=\frac{1}{2} \cdot C_{P} \cdot \rho A u^{3}
$$

Therefore, all parameters for turbine hydrodynamic performance are resolved by this methodology. A remark for this kind of flow is the stability and the solution image. Since the result is derived from a $4^{\text {th }}$ degree polynomial equation, there is a certain combination between Froude Number and by-pass velocity coefficient for the solution be real. This analysis is performed in (Houlsby et al., 2007). 


\section{ATTACHMENT E - Tidal Current Turbines}

This section is destined to show the differences between cross-flow and axial turbines for tidal current energy extraction. In the cross-flow section is shown the main advantages from this geometry and the basics of its hydrodynamics. In the axial flow section, some commercial turbines are shown with their performance parameters. The hydrodynamics of axial turbines is similar to wind turbines and could be understood by analytical methodologies such as with Blade Element Momentum Theory and the flow visualisation is given on the results from CFD analysis.

\section{Cross Flow Devices}

The most known cross flow device, that works for both wind and tidal current energy extraction is the Darrieus Machine, represented on the left from Figure 75 and it is a multidirectional turbine, which is considered an advantage for a turbine that depends on environmental flow, such wind that have not a constant direction. For tidal current turbines, in which the current direction is predictable, the main advantage of this turbine could be achieved for an adjustable axial device.

The velocity triangle, shown in Figure 75, presents a blade that works with negative angle of attack. The attack angle is composed mainly from the tangential speed of the blade section and by the incoming flow velocity. Assuming a constant inlet velocity, both attack angle and absolute velocity are variable along the rotation. Some designs use twisted blades to make the torque constant along the time for each blade.

Figure 75 - Cross flow tidal current turbine and its velocity triangle
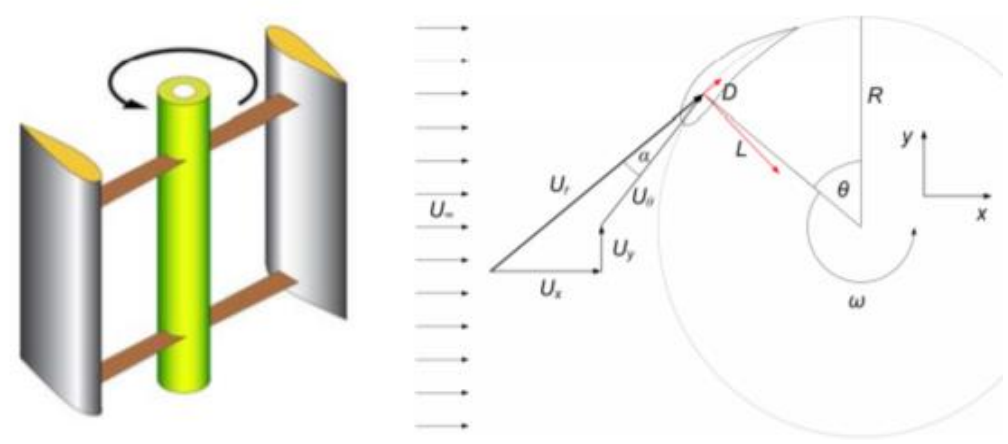

Source: (Consul, 2011) 


\section{Axial Devices}

An axial current turbine is very similar to a tradition wind turbine, since the rotation plane of the blades is orthogonal to the streamwise direction. Most of the researches on tidal current energy extraction focuses on such solution and prototipes are being deployied in a initial phase. The flow physics is similar to wind turbines and the basics from its aerodynamics is explained by the BEMT theory, that is often used in a preliminary design phase of wind turbines.

The axial device concept allow the blade pitch angle control in order to ajust the blades for the changes on the mean flow velocity. For harvesting energy from tides, this feature is necessary since the mean flow velocity changes according to the tidal cycles, being predictable and therefore controlable. In this manner, is possible to set up the blades position for the maximum energy extraction for each flow velocity. Besides of that, a rotational basis is possible in order to ajust the turbine axis according to the flow directionality.

The full scale turbines that are being deployed for tests and operation are from the axial kind, showing the preference for this kind of system.In the Figure 76 is shown the first turbine connect to the United Kingdom electrical grid. The device consists in two axial turbines with two blades each, with an output of $150 \mathrm{~kW}$ and was deployed in 2008. Another example of axial turbine that is being instaled come from Andritz Hydro, that is working on instalation of three devices of $1.5 \mathrm{MW}$ each in the Scotland Region (Andritz Hydro). Another comercial equipments are shown and compared in Table 12.

Figure 76 - Tidal current turbine being deployed in Strangford Lough

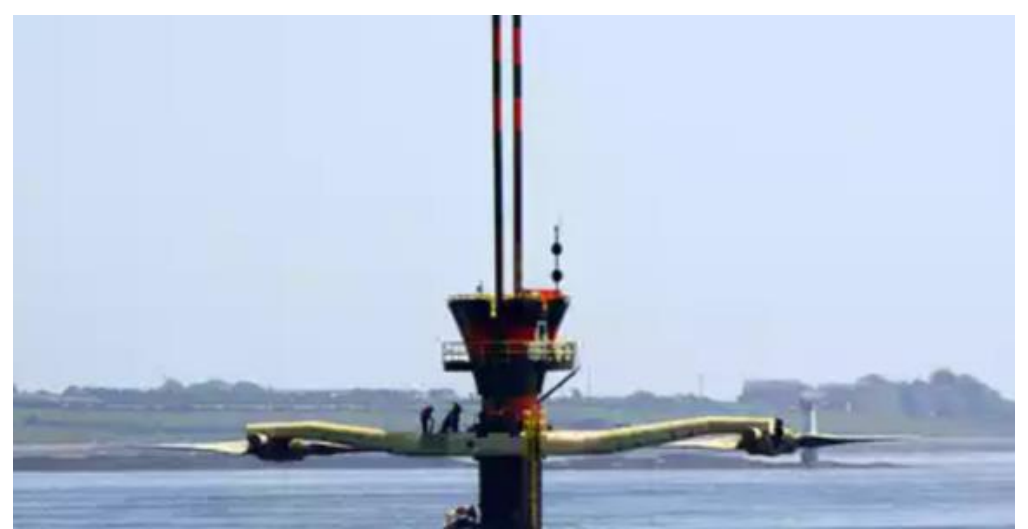

Source: (The Guardian, 2008) 
Table 12 - Axial tidal current turbines

\begin{tabular}{|c|c|c|c|c|}
\hline \multicolumn{2}{|l|}{ Turbine } & Maximum Power Output & Rotor Diameter & Operation Depth \\
\hline & $\begin{array}{l}\text { Andritz } \\
\text { Hammerfest }\end{array}$ & $2 \mathrm{MW}$ & $20 \mathrm{~m}$ & $\begin{array}{c}\text { There is no } \\
\text { available } \\
\text { information }\end{array}$ \\
\hline & $\begin{array}{l}\text { Voith HyTide } \\
1000\end{array}$ & $1 \mathrm{MW}$ & $13 \mathrm{~m}$ & $14 \mathrm{~m}$ \\
\hline & GE Oceade & $2 \mathrm{MW}$ & $18 \mathrm{~m}$ & $\begin{array}{c}\text { There is no } \\
\text { available } \\
\text { information }\end{array}$ \\
\hline & Seagen S & $\begin{array}{c}2 \mathrm{MW} \\
\text { each rotor }\end{array}$ & $20 \mathrm{~m}$ & $38 \mathrm{~m}$ \\
\hline & $\begin{array}{l}\text { Atlantis } \\
\text { Renewable } \\
\text { AR1600 }\end{array}$ & $1.5 \mathrm{MW}$ & $18 \mathrm{~m}$ & $\begin{array}{c}\text { There is no } \\
\text { available } \\
\text { information }\end{array}$ \\
\hline
\end{tabular}




\section{ATTACHMENT F - Finite Volume Elements}

\section{Finite Volume Method for Unstructured Meshes}

Based on the formulation from structured meshes, are developed the formulation for the unstructured meshes. Every element yields its own coordinate system, and from it, is derived the metrics relating the Cartesian coordinates to curvilinear coordinates, making use from the results obtained for structured meshes.

There are several kinds of elements that can be used for CFD, however, this text presents only the tetrahedral and hexahedral elements, both three-dimensional without the intermediate node. In the Figure 77 is shown a general element with "m" nodes. The properties on each element are interpolated according to the shape functions $\boldsymbol{n}_{\boldsymbol{i}}$.

$$
\phi=\sum_{i=1}^{m} n_{i}(x, y, z) . \phi_{i}
$$

The shape functions are equal to one on its correspondent node and zero at the others, according to the following equation:

$$
n_{i}\left(N_{j}\right)=\delta_{i j}
$$

Figure 77 - General Unstructured Element for Finite Volume Method
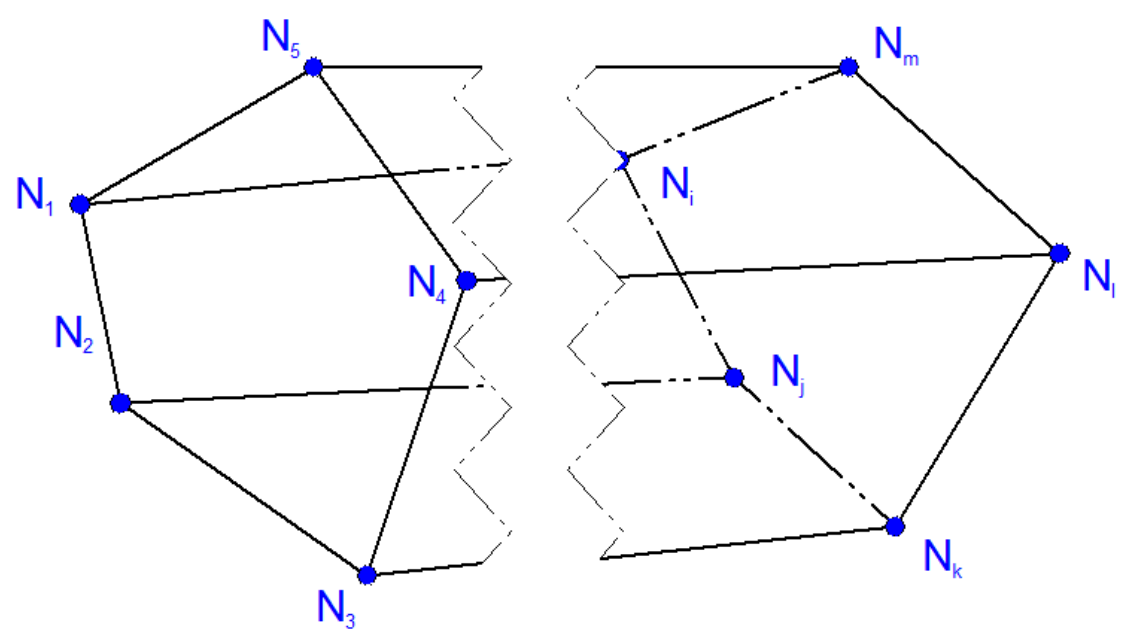


\section{Hexahedral Element}

For each hexahedral element is given a local coordinate system that can be related to the global coordinate system. For parametric elements and a simpler programming, is easier to calculate the coefficients associated with each variable in local coordinate and then transport by mapping functions to the global coordinates to assembly the system of equations in global coordinates. The shape functions, for a hexahedral element, shown in Figure 78, are given by:

$$
\begin{gathered}
n_{1}=(1-s)(1-t)(1-u) \\
n_{2}=s(1-t)(1-u) \\
n_{3}=s t(1-u) \\
n_{4}=(1-s) t(1-u) \\
n_{5}=(1-s)(1-t) u \\
n_{6}=s(1-t) u \\
n_{7}=s t u \\
n_{8}=(1-s) t u
\end{gathered}
$$

Figure 78 - Fluid Hexahedral Element

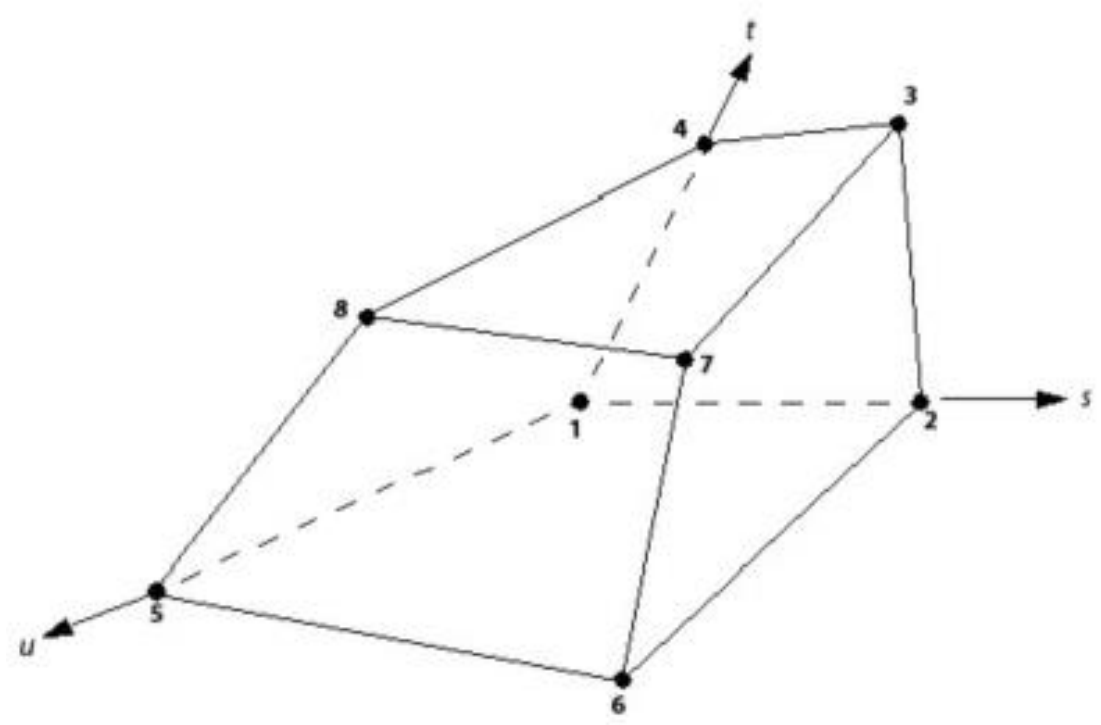

Source: (ANSYS Inc., 2016) 
Tetrahedral Element

The main advantage of the tetrahedral element is that it has a better adaptation for complex domains. However, due to its adaptability, it often presents distorted shapes with poor aspect ratios. This happens mainly if the growth factor is high in the transition between refined regions with coarser regions. Intermediate elements such as wedges and pyramids are used together with hexahedral elements in transition regions, however, this formulation will not be presented in this text. For the tetrahedral element, shown in the

Figure 79, the shape functions presented on the equation (F.2), providing a linear interpolation, differently of the shape functions from the hexahedral elements. The other 3D elements, cited on this text have hybrid shape functions, being linear for some nodes and non-linear for other.

$$
\begin{gathered}
n_{1}=1-s-t-u \\
n_{2}=s \\
n_{3}=t \\
n_{4}=u
\end{gathered}
$$

Figure 79 - Fluid Tetrahedral Element

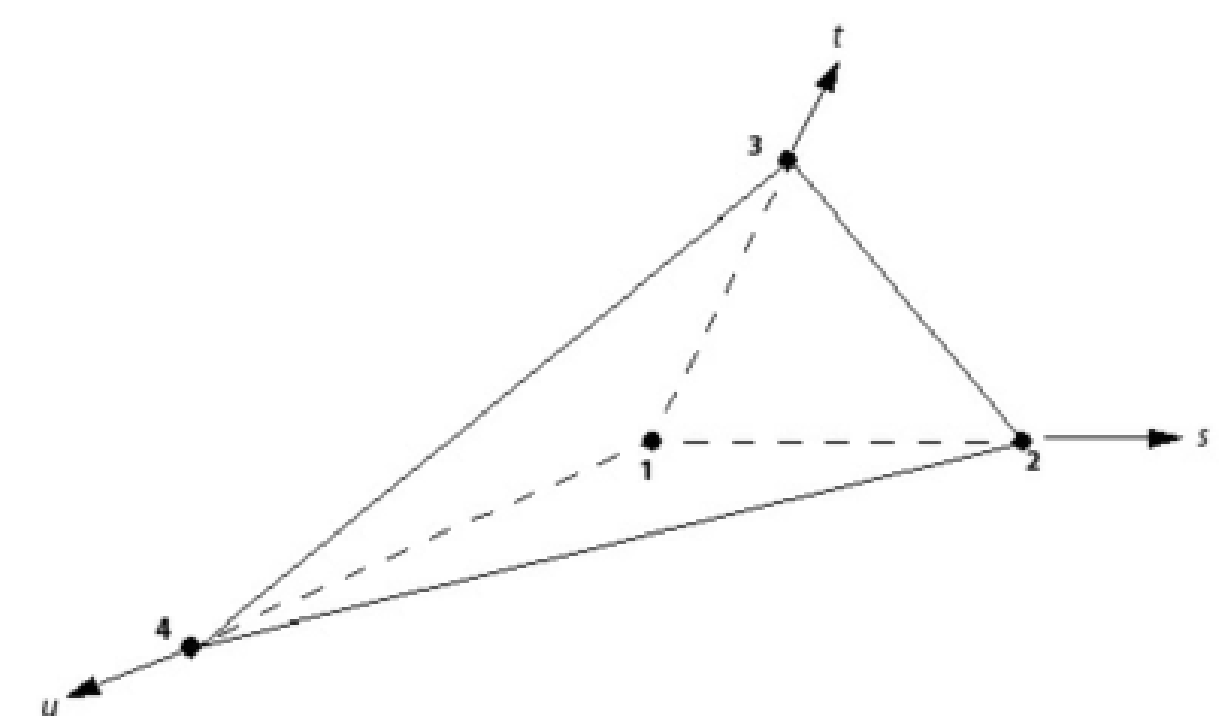

Source: (ANSYS Inc., 2016) 